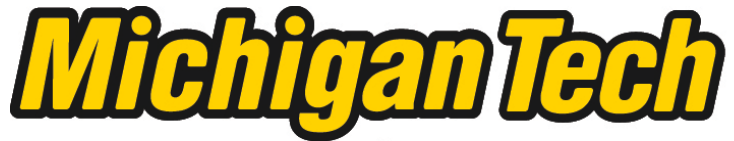 \\ Michigan Technological University Create the Future Digital Commons @ Michigan Tech
}

Dissertations, Master's Theses and Master's Reports - Open

Dissertations, Master's Theses and Master's

Reports

2012

\section{Large Eddy Simulation of dispersed multiphase flow}

Yejun Gong

Michigan Technological University

Follow this and additional works at: https://digitalcommons.mtu.edu/etds

Part of the Applied Mathematics Commons, and the Mechanical Engineering Commons Copyright 2012 Yejun Gong

\section{Recommended Citation}

Gong, Yejun, "Large Eddy Simulation of dispersed multiphase flow", Dissertation, Michigan Technological University, 2012.

https://doi.org/10.37099/mtu.dc.etds/729

Follow this and additional works at: https://digitalcommons.mtu.edu/etds

Dart of the Applied Mathematics Commons, and the Mechanical Engineering Commons 
Gong, Yejun

\begin{abstract}
A DISSERTATION
Submitted in partial fulfillment of the requirements for the degree of DOCTOR OF PHILOSOPHY (Computational Science \& Engineering)
\end{abstract}

MICHIGAN TECHNOLOGICAL UNIVERSITY

2012

(c) 2012 Gong, Yejun 

This dissertation, "Large Eddy Simulation of Dispersed Multiphase Flow," is hereby approved in partial fulfillment of the requirements for the Degree of DOCTOR OF PHILOSOPHY IN COMPUTATIONAL SCIENCE \& ENGINEERING.

Department of Mathematical Science

Signatures:

Dissertation Advisor

Dr. Tanner, Franz X

Committee Member

Dr. Feigl, Kathleen A

Committee Member

Dr. Yang, Song Lin

Committee Member

Dr. Kolkka, Robert W

Department Chair

Dr. Gockenbach, Mark S

Date 



\title{
Dedication
}

"Large Eddy Simulation of Dispersed Multiphase Flow"

Ph.D. thesis by Yejun Gong,

Dedicated to

\author{
My parents
}

and

teachers and friends and

in loving memory of my grandparents! 


\section{Contents}

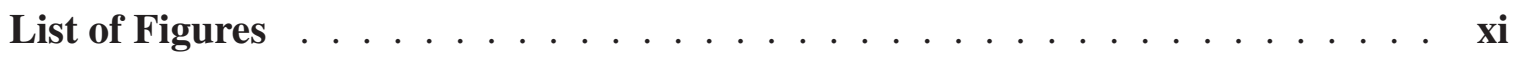

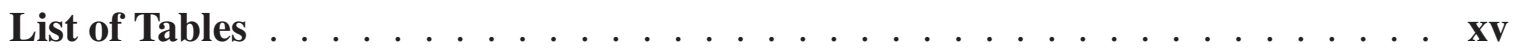

Acknowledgments $\ldots \ldots \ldots \ldots \ldots \ldots \ldots \ldots \ldots \ldots \ldots \ldots \ldots \ldots$

ABBREVIATIONS $\ldots \ldots \ldots \ldots \ldots \ldots \ldots \ldots \ldots \ldots \ldots$ viii

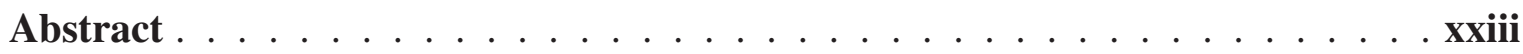

1 Introduction $\ldots \ldots \ldots \ldots \ldots \ldots \ldots \ldots \ldots \ldots \ldots$

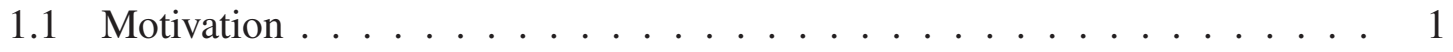

1.2 Research Objectives . . . . . . . . . . . . . . . . . . 3

2 Background ......................... 4

2.1 Governing equations $\ldots \ldots \ldots \ldots \ldots \ldots \ldots \ldots \ldots \ldots$

2.1.1 Mass Balance Equation . . . . . . . . . . . . . . . 4

2.1.2 Momentum Balance Equation . . . . . . . . . . . . 5

2.1.3 Energy Balance Equation . . . . . . . . . . . . . . . . . 7

2.2 Introduction to turbulence . . . . . . . . . . . . . . 7

2.2.1 The physical nature of turbulence $\ldots \ldots \ldots \ldots$

2.2.2 Overview of turbulence solution approaches . . . . . . . . . 11

2.2.2.1 Direct numerical simulation . . . . . . . . . . . . 11

2.2.2.2 Reynolds-averaged Navier-Stokes method . . . . . . . . . 13

2.2.2.3 Large eddy simulation . . . . . . . . . . . . . . . 14

2.3 Reynolds-averaged Navier-Stokes method . . . . . . . . . . . . . 15

2.3.1 Time averaging . . . . . . . . . . . . . . 15

2.3.1.1 Reynolds time averaging . . . . . . . . . . . . 15

2.3.1.2 Favre time averaging . . . . . . . . . . . 16

2.3.2 Time-averaged Navier-Stokes equations . . . . . . . . . . . 17

2.3.3 RANS closure problem . . . . . . . . . . . . . . . . . 18

2.3.3.1 Standard $k-\varepsilon$ model . . . . . . . . . . . . . . . 18

2.3.3.2 RNG $k-\varepsilon$ model . . . . . . . . . . . . 20

2.4 Large eddy simulation . . . . . . . . . . . . . . . . . . 20

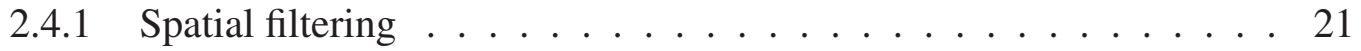


2.4.2 Spatial-filtered Navier-Stokes equations . . . . . . . . . . . . 24

2.4.3 Subgrid-scale closure models . . . . . . . . . . . . . . . 24

2.4.3.1 Smagorinsky model . . . . . . . . . . . . . . 25

2.4.3.2 LES one-equation model . . . . . . . . . . . . . 26

2.5 Computational Aspects . . . . . . . . . . . . . . . . . 27

2.5 .1 Computation code . . . . . . . . . . . . . . . 28

2.5 .2 Discretization . . . . . . . . . . . . . . . . . . . . . . . 29

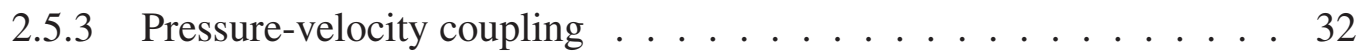

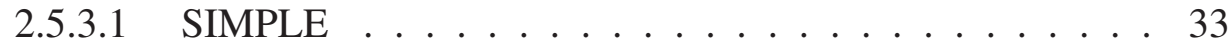

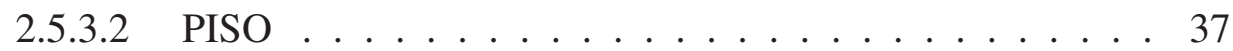

3 Simulation of flows over a backward-facing step . . . . . . . . . 40

3.1 Introduction . . . . . . . . . . . . . . . . 40

3.2 Flow configuration and initial conditions . . . . . . . . . . . . 42

3.3 LES/RANS results and discussion . . . . . . . . . . . . . . . 44

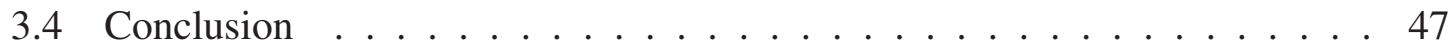

4 Simulation of fuel sprays $\ldots \ldots \ldots \ldots \ldots$

4.1 Liquid phase modeling . . . . . . . . . . . . . . . 51

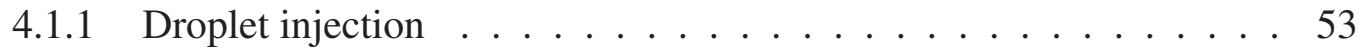

4.1 .2 Droplet breakup . . . . . . . . . . . . . . . . . . . 54

4.1 .3 Droplet drag . . . . . . . . . . . . . . . . . . 56

4.1 .4 Droplet evaporation . . . . . . . . . . . . . . 58

4.1.5 Droplet dispersion . . . . . . . . . . . . . . . . 60

4.1.6 Droplet collision . . . . . . . . . . . . . . . . 61

4.2 Gaseous phase modeling . . . . . . . . . . . . . 62

4.3 Test conditions . . . . . . . . . . . . . . . . . . 65

4.4 LES/RANS results and discussion $\ldots \ldots \ldots$. . . . . . . . . . 67

4.4 .1 Non-evaporating sprays . . . . . . . . . . . . . 67

4.4.1.1 Spray tip penetration of Cases 1-3 . . . . . . . . . 69

4.4.1.2 Comparison between RANS and LES . . . . . . . . . 70

4.4 .2 Evaporating sprays $\ldots \ldots \ldots \ldots \ldots$

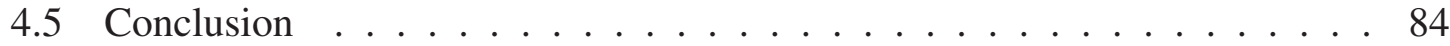

5 Hydrotreated vegetable oil (HVO) sprays . . . . . . . . . . . . . 86

5.1 Introduction . . . . . . . . . . . . . . . . . . 87

$5.1 .1 \quad$ ReFuel project . . . . . . . . . . . . . . . 87

5.1 .2 Fuel spray properties $\ldots \ldots \ldots \ldots 8$

5.2 Computation details . . . . . . . . . . . . . . . . . 92

5.3 LES/RANS results and discussions . . . . . . . . . . . . . . 93

5.3 .1 Non-evaporating sprays . . . . . . . . . . . . . . . 93

5.3 .2 Evaporating spray . . . . . . . . . . . . . . . . . . . 99

5.4 Conclusion . . . . . . . . . . . . . . . . . . . . . . 109 


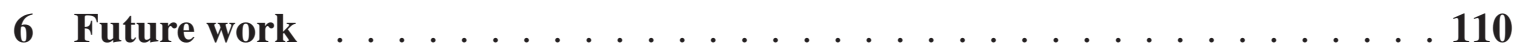

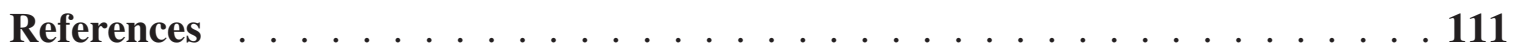

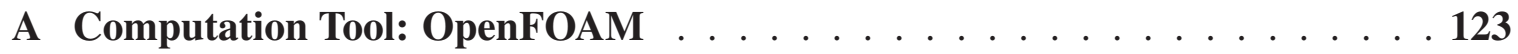

A.1 New dieselSpray Submodels . . . . . . . . . . . . . . . . . . 123

A.1.1 IDSD: power law . . . . . . . . . . . . . . . . . 123

A.1.2 Atomization Model: Levich . . . . . . . . . . . . . . . . 124

A.1.3 Breakup Model: CAB . . . . . . . . . . . . . . . . . . 126

A.2 dieselLES: diesel spray LES solver . . . . . . . . . . . . . . 127

A.3 New Fuel Realization . . . . . . . . . . . . . . . . . . . . . . 129

A.4 Compile the user-defined libraries . . . . . . . . . . . . . . . 132

A.4.1 Move an existed library to user's directory . . . . . . . . . . . 132

A.4.2 Add a new library or library components . . . . . . . . . . 133

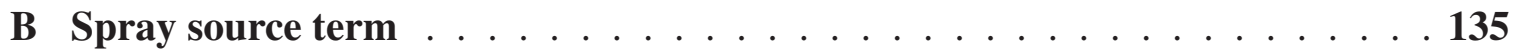

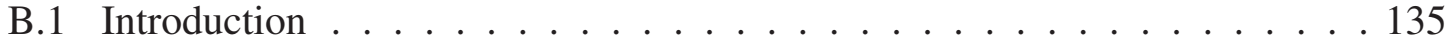

B.2 Simulations and Results . . . . . . . . . . . . . . . . . . 137

B.3 Discussion . . . . . . . . . . . . . . . . . . . . . . . 140

B.4 OpenFOAM Realization . . . . . . . . . . . . . . . . . . . 140

B.4.1 Add a subfield sks to dieselSpray class . . . . . . . . . . . . 141

B.4.2 Add a member function updateSpraySource to class RASModel . 144

B.4.3 Add the spray source term sks_to $k-\varepsilon$ equations . . . . . . . . 146

B.4.4 Update the spray source term in solvers . . . . . . . . . . . . 147 


\section{List of Figures}

2.1 Turbulence models ordered according to their decreasing level of complexity. Abbreviations: RST = Reynolds-Stress Transport models; ARS = Algebraic Reynolds-Stress models. . . . . . . . . . . . . . . . . . 13

3.1 Backward-facing step geometry, ${ }^{1} S=4.9 \mathrm{~mm}, h=5.2 \mathrm{~mm}, H=10.1 \mathrm{~mm}, \mathrm{~W}=$ $5 \mathrm{~mm}, L=300 \mathrm{~mm} \ldots \ldots \ldots \ldots \ldots$

3.2 2D Nonuniform Mesh, $8 \times 22$ before step and $312 \times 40$ after step. . . . . . . . . 43

3.3 Experimental and computed velocity profiles for $\mathrm{Re}=389$ at different

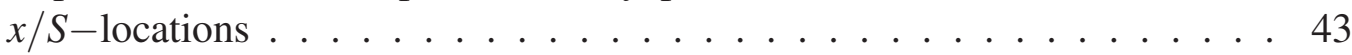

3.4 Velocity near the lower wall for $R e=389$. Experimental reattachment point, •; LES: Laminar, ॰-; Smagorinsky, $\diamond-$; OneEq, •-; RANS: Lami-

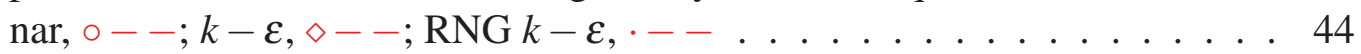

3.5 Experimental and computed velocity profiles for $\mathrm{Re}=1000$ at different $x / S$-locations . . . . . . . . . . . . . . . . . . 47

3.6 Velocity near the lower wall for $\mathrm{Re}=1000$. Experimental reattachment point, •; LES: Laminar, ॰-; Smagorinsky, $\diamond-$; OneEq, •-; RANS: Lami-

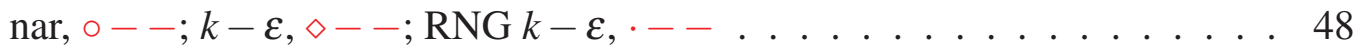

3.7 Velocity color plot of $u_{x}$ for $\mathrm{Re}=1000 \ldots \ldots$. . . . . . . . . . . . 48

3.8 Velocity near the top wall for $\mathrm{Re}=1000$. Experimental detachment point, $\bullet$; LES: Laminar, ॰-; Smagorinsky, $\diamond-;$ OneEq, ·-; RANS: Laminar, ॰ - ; $k-\varepsilon, \diamond--; \operatorname{RNG} k-\varepsilon, \cdot-\ldots \ldots \ldots . \ldots . \ldots 48$

4.1 Computation domain. . . . . . . . . . . . . . . . . 66

4.2 Temporal change in spray tip penetration with different grid size for RANS simulation. . . . . . . . . . . . . . . . . . . 68

4.3 Temporal change in spray tip penetration with different grid size for LES simulation. . . . . . . . . . . . . . . . . . . 68

4.4 Temporal change in spray tip penetration for RANS and LES using DF2. . . 70

4.5 Gas phase velocity vector at cross section for RANS Case 1 at time $0.0024 \mathrm{~s}$. . . . 71

4.6 Gas phase velocity vector at cross section for LES Case 1 at time $0.0024 \mathrm{~s}$. . . . . 71

4.7 Cross-sectional plot for RANS Case 1 colored by magnitude of vorticity at time 0.0024 s. . . . . . . . . . . . . . . . . . 72

4.8 Cross-sectional plot for LES Case 1 colored by magnitude of vorticity at time 0.0024 s. . . . . . . . . . . . . . . . . 72 
4.9 Cross-sectional plot for RANS Case 1 colored by turbulent kinetic energy at time 0.0024 s. . . . . . . . . . . . . . . . . . 73

4.10 Cross-sectional plot for LES Case 1 colored by turbulent kinetic energy at time 0.0024 s. . . . . . . . . . . . . . . . . . 73

4.11 Turbulent kinetic energy $k$ versus nozzle distance along centerline of Case 1. 73

4.12 Cross-sectional plot for RANS Case 1 colored by the magnitude of the fluid velocity at time $0.0024 \mathrm{~s} . \ldots \ldots$. . . . . . . . . . . . . . . 75

4.13 Cross-sectional plot for LES Case 1 colored by the magnitude of the fluid velocity at time $0.0024 \mathrm{~s} . \ldots \ldots \ldots$. . . . . . . . . . . . . . . 75

4.14 Cross-sectional SMD at time $0.0024 \mathrm{~s}$ for Case $1 \ldots$. . . . . . . . . . . . . 75

4.15 Spray plot for RANS Case 1 colored by droplet diameter at time $0.0024 \mathrm{~s}$. . . . . 77

4.16 Spray plot for LES Case 1 colored by droplet diameter at time $0.0024 \mathrm{~s}$. . . . . . 77

4.17 Temporal change in spray tip penetration for RANS and LES evaporating case using HEP in evaporating case. . . . . . . . . . . . . . . 77

4.18 Gas phase velocity vector at cross section for RANS evaporating case at time 0.0008 s. . . . . . . . . . . . . . . . . . . . . . 79

4.19 Gas phase velocity vector at cross section for LES evaporating case at time 0.0008 s. . . . . . . . . . . . . . . . . . . . 79

4.20 Turbulent kinetic energy $k$ versus nozzle distance along centerline of evaporating case. . . . . . . . . . . . . . . . . . . . 80

4.21 Cross-sectional plot for RANS evaporating case colored by turbulent kinetic energy at time $0.0016 \mathrm{~s} . \ldots \ldots$. . . . . . . . . . . . . . . 81

4.22 Cross-sectional plot for LES evaporating case colored by turbulent kinetic energy

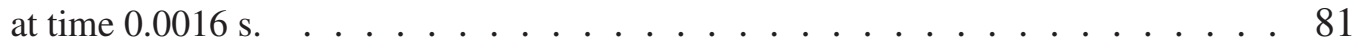

4.23 Cross-sectional SMR at time $=0.0016 \mathrm{~s}$ for evaporating case . . . . . . . . . . . . 81

4.24 Spray plot for RANS evaporating case colored by droplet diameter at time 0.0008 s. . . . . . . . . . . . . . . . . . . 83

4.25 Spray plot for LES evaporating case colored by droplet diameter at time $0.0008 \mathrm{~s} . \quad 83$

4.26 Spray plot for RANS evaporating case colored by droplet diameter at time 0.0016 s. . . . . . . . . . . . . . . . . 83

4.27 Spray plot for LES evaporating case colored by droplet diameter at time $0.0016 \mathrm{~s} . \quad 83$

5.1 Fuel properties comparison. . . . . . . . . . . . . . . . . . 90

5.2 Momentum relaxation time $\tau$ of the non-evaporating Case 1 with $\mu_{g}=15.11 \mathrm{~m}^{2} / \mathrm{s}$ for a drop with diameter $d_{0}=0.2 \mathrm{~mm}$ and the injection velocity. . . . . . . . . . 91

5.3 Thermal diffusivity $\alpha=\frac{K}{\rho c_{p}}$ and $\mathrm{K}$ is the thermal conductivity. . . . . . . . . . 91

5.4 Temporal change in spray tip penetration of non-evaporating sprays with different fuels for the RANS and LES simulations. . . . . . . . . . . . . . . . 93

5.5 Cross-sectional SMD of the non-evaporating cases at the injection time $=0.0024 \mathrm{~s}$ for the RANS simulations. . . . . . . . . . . . . . . . . 94

5.6 Cross-sectional SMD of the non-evaporating cases at the injection time $=0.0024 \mathrm{~s}$ for the LES simulations. . . . . . . . . . . . . . . . 94 
5.7 Spray droplet diameter in $\mathrm{m}$ of the non-evaporating Case 1 using different fuels at time $=0.0024 \mathrm{~s}$ for the RANS and LES simulations. . . . . . . . . . . . . . . 96

5.8 Magnitude of axial velocity versus nozzle distance along the centerline of the nonevaporating Case 1 at time $=0.0024$ s. . . . . . . . . . . . . . . . . . 97

5.9 Turbulent viscosity $\mu_{t}$ versus nozzle distance along the centerline of the nonevaporating Case 1 at time $=0.0024$ s. . . . . . . . . . . . . . . . . . . . 97

5.10 Turbulent kinetic energy $k$ versus nozzle distance along the centerline of the nonevaporating Case 1 at time $=0.0024 \mathrm{~s}$. . . . . . . . . . . . . . . . 97

5.11 Turbulent kinetic energy in $\mathrm{m}^{2} / \mathrm{s}^{2}$ of the non-evaporating Case 1 using different fuels at time $=0.0024 \mathrm{~s}$ for the RANS and LES simulations. . . . . . . . . . . . 98

5.12 Magnitude of vorticity in $s^{-1}$ of the non-evaporating Case 1 using different fuels at time $=0.0024 \mathrm{~s}$ for the RANS and LES simulations. . . . . . . . . . . . . . 100

5.13 Temporal change in spray tip penetration of evaporating sprays with different fuel for the RANS and LES simulations. . . . . . . . . . . . . . . . . . . . . 101

5.14 Spray droplet temperature in $\mathrm{K}$ of the evaporating case using different fuels at time $=0.0008 \mathrm{~s}$ for the RANS and the LES simulations. . . . . . . . . . . . . 102

5.15 Spray droplet diameter in $\mathrm{K}$ of the evaporating case using different fuels at time $=0.0016 \mathrm{~s}$ for the RANS and the LES simulations. . . . . . . . . . . . . 103

5.16 Gas phase fuel mass fraction of the evaporating case using different fuels at $0.0008 \mathrm{~s}$ for the RANS and the LES simulations. . . . . . . . . . . . . . . 104

5.17 Gas phase fuel mass fraction of the evaporating case using different fuels at $0.0016 \mathrm{~s}$ for the RANS and the LES simulations. . . . . . . . . . . . . . . 105

5.18 Cross-sectional SMD of the evaporating cases at time $=0.0008 \mathrm{~s}$ for the RANS and LES simulations. . . . . . . . . . . . . . . . . . . 108

5.19 Magnitude of axial gas velocity versus nozzle distance along the centerline of the evaporating cases at $0.0008 \mathrm{~s}$ and $0.0016 \mathrm{~s}$ for the RANS and LES simulations. . . 108

A.1 Penetration curve of one tuning case: the blue line with the kink is the result of the OpenFOAM origin code, and the red line is the result of the modified code . . . . . . . . . . . . . . . . . 125

B.1 Spray tip penetration versus time of the non-evaporating cases for DF2. . . 137

B.2 Axial turbulent kinetic energy $k$ of the non-evaporating Case 1 for DF2 . . . . . 138

B.3 Axial turbulent viscosity $\mu_{t}$ of the non-evaporating Case 1 for DF2 . . . . . . . 138

B.4 Vapor penetrations (dashed lines) and liquid penetrations (solid lines) versus time of the evaporating case for HEP. . . . . . . . . . . . . . . . . 138

B.5 Axial turbulent kinetic energy $\mathrm{k}$ of the evaporating case for HEP . . . . . . . . . 139

B.6 Axial turbulent viscosity $\mu_{t}$ of the evaporating case for HEP . . . . . . . . . . . 139

B.7 Spray droplet diameter in $\mathrm{m}$ of the non-evaporating Case 1 using DF2 fuel at 0.0012 s: (a) $S_{k}=0$; (b) $S_{k} \neq 0 \ldots \ldots$. . . . . . . . . . . . . . . . . . . .

B.8 Turbulent kinetic energy in $\mathrm{m}^{2} / \mathrm{s}^{2}$ of the non-evaporating Case 1 using DF2 fuel at $0.0012 \mathrm{~s}$ : (a) $S_{k}=0$; (b) $S_{k} \neq 0$. 


\section{List of Tables}

2.1 The values of $\phi$ and $\Gamma$ in the Navier-Stokes equations . . . . . . . . . . . 29

4.1 Experimental settings . . . . . . . . . . . . . . . . 66

4.2 Computation Time of Cold Case $1 \ldots \ldots$. . . . . . . . . . . 69

5.1 Fuel properties related to the spray performance. . . . . . . . . . 88

A.1 Temperature Independent Thermal Physical Properties . . . . . . . . . . 129 


\section{Acknowledgments}

First and foremost, I would like to express my gratitude to Professor Franz Tanner for accepting me into the Computational Science \& Engineering (CSE) PhD program at MTU and being a very patient advisor.

I also would like to thank Prof. Mark S Gockenbach for the financial help from the department, and Prof. Phil Merkey for the help from the CSE program. The help from Prof. Kathleen A Feigl, Prof. Song Lin Yang and Prof. Robert W Kolkka for providing me with insight into Computational Fluid Dynamics and Large Eddy Simulation are also highly appreciated.

The financial support from the Finnish Funding Agency for Technology and Innoviation (TEKES) and the Internal Combustion Engine Research Group at Helsinki University of Technology during my six months CPT in Finland is also appreciated. In addition, I would like to thank Prof. Martti Larmi, Dr. Ossi Kaario and Dr. Ville Vuorinen for their help with the hydrotreated vegetable oil (HVO) project.

Finally I would like to thank all my friends and family for their support. 


\section{ABBREVIATIONS}

\section{Acronyms}

DF2 Diesel fuel $2(\mathrm{C} 12 \mathrm{H} 26)$.

DNS Direct numerical simulation.

GTL Gas-to-liquids diesel fuel.

HEP n-heptane (C7H16).

HVO Hydrotreated vegetable oil.

LES Large eddy simulation.

RANS Reynolds-averaged Navier-Stokes simulation.

\section{Non-dimensional parameters}

$\begin{array}{ll}\text { Co } & \text { Courant number. } \\ \operatorname{Pr} & \text { Prandtl number. } \\ R e & \text { Reynolds number. } \\ R e_{t} & \text { Turbulent Reynolds number. } \\ W e & \text { Weber number. }\end{array}$

\section{Greek symbols}

$\Delta \quad$ Filter width. 


$\begin{array}{ll}\mu & \text { Dynamic viscosity, molecular viscosity. } \\ \mu_{t} & \text { Eddy viscosity, turbulent viscosity. } \\ v & \text { Kinematic viscosity. } \\ \Omega & \text { Entire flow domain. } \\ \rho & \text { Density. } \\ \tau^{R} & \text { Reynolds viscous stress tensor. } \\ \tau^{S} & \text { Subgrid-scale stress tensor. } \\ \tau_{i j} & \text { Viscous stress tensor. } \\ \varepsilon & \text { Dissipation Rate. } \\ K & \text { Thermal conductivity. } \\ \eta & \text { Kolmogorov length scale. }\end{array}$

\section{Roman symbols}

$\begin{array}{ll}C d & \text { Drag coefficient. } \\ d & \text { Drop diameter. } \\ h & \text { Enthalpy. } \\ k & \text { Turbulent kinetic energy. } \\ y & \text { Drop deformation. } \\ c_{p} & \text { Specific heat. } \\ E & \text { Total energy. } \\ G & \text { Spatial filter. } \\ \mathbf{g} & \text { Body force per unit mass. } \\ g_{i} & \text { i-th component of the body force per unit mass. } \\ h_{l} & \text { Heat of vaporization. }\end{array}$




$\begin{array}{ll}L & \text { Integral scale, characteristic length. } \\ \omega & \text { Drop oscillation frequency. } \\ p & \text { Pressure. } \\ P & \text { Modified pressure. } \\ p_{v} & \text { Vapor pressure. } \\ \mathbf{q} & \text { Heat flux vector. } \\ \sigma & \text { Surface tension. } \\ S & \text { Strain rate tensor. } \\ T & \text { Static temperature. } \\ \alpha & \text { Thermal diffusivity. } \\ \mathbf{u} & \text { Fluid velocity. } \\ u_{j} & \text { j-th component of the fluid velocity. } \\ U & \text { Characteristic velocity or the magnitude of the velocity. } \\ x_{j} & \text { Position vector. } \\ \end{array}$

\section{Subscripts}

$\begin{array}{ll}d & \text { Drop. } \\ g & \text { Gas. } \\ l & \text { Liquid. } \\ b u & \text { Breakup. } \\ r e l & \text { Relative. } \\ t & \text { Turbulent. }\end{array}$




\section{Superscripts}

$\begin{array}{ll}\text { lam } & \text { Laminar. } \\ \text { sgs } & \text { Subgrid-scale. } \\ \text { tot } & \text { Total. } \\ \text { tub } & \text { Turbulent. }\end{array}$

\section{Auxiliary symbols}

$\overline{f^{0}}=f \quad$ The time average part of a generic variable $f^{0}$.

$\widetilde{f^{0}}=f \quad$ The spatial filtered part of a generic variable $f^{0}$.

$f^{\prime} \quad$ The fluctuation part of a generic variable $f^{0}$.

$\left|f^{o}\right| \quad$ The magnitude of a generic variable $f^{o}$. 



\section{Abstract}

This thesis covers two main topics. The first is the comparison between the Reynoldsaveraged Navier-Stokes (RANS) simulation and the Large Eddy Simulation (LES) of high injection pressure diesel sprays under non-evaporating or evaporating conditions. The second topic is the comparison of the fuel behavior in the spray process between the hydrotreated vegetable oil (HVO) and the conventional EN 590, diesel \#2 and n-heptane fuels.

To validate the RANS and LES spray simulations, comparisons were made with experimental data. The LES turbulence model, the initial drop size distribution (IDSD), the Levich jet breakup model and the $\mathrm{CAB}$ drop breakup model are realized into the OpenFOAM code. The liquid phase models, including the drop atomization, breakup, collision and evaporating models, are tested and tuned such that the simulated penetration curves are near to the experimental ones. The spray penetration, fuel velocity, turbulent kinetic energy, turbulent viscosity, vorticity and drop size distribution are investigated and compared for the different computation cases.

In addition, the HVO fuel and EN 590 fuel are added in to the OpenFOAM fuel libraries. The physical properties of the four investigated fuels are compared and their influence on the performance of the fuels in the spray simulations are investigated.

Keywords: Spray, Large eddy simulation, Reynolds-averaged Navier-Stokes Method, Computational Fluid Dynamics, Hydrotreated vegetable fuel 


\section{Introduction}

\subsection{Motivation}

During the last several decades, the method of Large Eddy Simulation (LES) has become increasingly popular in Computational Fluid Dynamics (CFD). The reason for this development is with the improvement of the ever increasing computation power of digital comput-

ers. From the first LES model proposed by Smagorinsky ${ }^{2}$ in 1963, LES has been developed to serve the significant difficult engineering problems with transient, multi-phase, turbulent flows.

The Reynolds-averaged Navier-Stokes (RANS) method is another popular approach in modeling turbulent phenomenon. The RANS approach resolves the time-averaged NavierStokes (NS) equations, while the LES approach resolves the spatially-averaged (filtered) NS equations. Both approaches produce turbulence fluctuation terms that need to be modeled. The main advantage of LES over the RANS is the increased ability in simulations of the transient phenomena. ${ }^{3}$ Though the RANS is much more computationally efficient, the time-averaged NS equations are inadequate to resolve the instantaneous flow characteristics. The LES approach is better than the RANS approach in capturing the unsteady effects, and LES is not so computationally expensive as Direct Numerical Simulation (DNS). 
Hence, LES is considered as one of the most promising approaches in modeling the unsteady flows of high Reynolds number turbulence.

Both the RANS and LES approaches separate the variables into the averaged parts and the fluctuation parts. The averaged parts are resolved and describe the large-scale behavior of the turbulent flows. In RANS, the time varying fluctuation terms are modeled and represent the whole range of turbulence. In LES, the spatially filtered terms represent the large scale turbulence, while the small scales are modeled. These small scale eddies are associated with the high frequency fluctuations. Thus, LES only models the high frequency turbulence, while RANS models all frequencies of turbulence. The difficulty in modeling the whole range of turbulence leads to the failure of RANS in flows with large-scale vortical structures.

LES preforms much better than RANS in the computing regions with high frequency of turbulent fluctuations. However, on the other hand, LES consumes much more computation resources than RANS, especially for the near wall regions. The assumption of isotropy in LES of small scales is less valid near walls, and high levels of mesh refinement are required.

In the present study, the advantages of LES over RANS in transient flows are discussed by comparing the two popular turbulence modeling methods in the benchmark backwardfacing step problem and the fuel spray simulations. 


\subsection{Research Objectives}

Besides the comparison between RANS and LES, the main objective of this thesis is to test and apply the LES in fuel sprays of a typical turbulent flow. The key to modeling turbulent flows is to account for the increase in the diffusive transport properties of the flow by introducing an eddy or turbulence viscosity. This is analogous to the molecular viscosity which describes the momentum transfer between flow layers in laminar flows. In

this study, we consider two RANS eddy viscosity turbulence models, the standard $k-\varepsilon^{4}$ and the RNG $k-\varepsilon$ models, ${ }^{5,6}$ as well as two LES eddy viscosity models, the Smagorinsky ${ }^{2}$ and the one-equation (OneEq) models. ${ }^{7}$

The thesis is organized as follows. Chapter 2 gives an introduction of the background of LES, and reviews the literatures on this subject. Chapter 3 compares RANS and LES in the laminar limit of the benchmark backward-facing step simulation. Chapter 4 compares RANS and LES for the non-evaporating sprays and evaporating spray. Chapter 5 uses RANS and LES to compare the performance of hydrotreated vegetable oil (HVO) and several other fuels in the spray process. Finally, Chapter 6 contains the conclusions and the future work. 


\section{Background}

\subsection{Governing equations}

Fluid flows are governed by the conservation laws for mass, momentum and energy. Applying these laws to a fluid element generates the three governing equations: the mass balance (or continuity) equation, the momentum balance (or Navier-Stokes) equation, and the energy balance equation. In the following, these equations are briefly discussed. A detailed derivation can be found in Ref. ${ }^{8}$ Throughout this thesis the Einstein summation convention is employed, that is, $\sum_{i=1}^{n} a_{i} b_{i}$ is written as $a_{i} b_{i}$, where the range of $i, i=1, \cdots, n$, is implicitly assumed.

\subsubsection{Mass Balance Equation}

By the conservation law of mass, the rate of change of the mass in an infinitesimal fixed control volume just depends on the rate of mass flux passing out of the surface of the control volume. The control volume is defined as a region of space containing the fluid which is selected as is most convenient. ${ }^{9}$ Then the continuity equation is obtained as

$$
\frac{\partial \rho}{\partial t}+\frac{\partial}{\partial x_{j}}\left(\rho u_{j}\right)=0,
$$


where $\rho$ denotes the fluid density, $x_{j}$ is the $j^{\text {th }}$ component of the cartesian coordinates and

$u_{j}$ represents the $j^{t h}$ component of the fluid velocity. To the left side of Eq. (2.1), the first term describes the rate of increase of the mass per control volume, and the second term represents the rate of mass flux passing out of the control surface per unit volume. ${ }^{10}$

\subsubsection{Momentum Balance Equation}

Applying the momentum balance law (Newton's second Law) to a fluid passing through an infinitesimal fixed control volume generates the momentum equation

$$
\frac{\partial}{\partial t}\left(\rho u_{i}\right)+\frac{\partial}{\partial x_{j}}\left(\rho u_{j} u_{i}\right)=\rho g_{i}-\frac{\partial p}{\partial x_{i}}+\frac{\partial \tau_{i j}}{\partial x_{j}}
$$

Here $p$ denotes the pressure, $g_{i}$ represents the $i^{t h}$ component of the body force $\mathbf{g}$. , and $\tau=\left(\tau_{i j}\right)$ denotes the stress tensor.

The left side of Eq. (2.2) is a time derivative term plus a convection term. The first time derivative term describes the rate change of momentum per unit control volume, and the second convection derivative term represents the rate of momentum lost through the surface of the control volume. To the right-hand side of Eq. (2.2), the first term is the body force per unit volume, and the other terms represent the surface forces per unit volume. 
It is convenient to combine the body force and pressure gradient term by defining a new variable called modified pressure. It is defined as,

$$
P \equiv p+\rho g z
$$

where $g=g_{z}$ is the gravity acceleration and $z=x_{z}$ is the cartesian coordinate in $z$ direction. Then Eq. (2.2) can be written as,

$$
\frac{\partial}{\partial t}\left(\rho u_{i}\right)+\frac{\partial}{\partial x_{j}}\left(\rho u_{j} u_{i}\right)=-\frac{\partial P}{\partial x_{i}}+\frac{\partial \tau_{i j}}{\partial x_{j}}
$$

where the stress tensor $\tau_{i j}$ is given by

$$
\tau_{i j}=-\frac{2}{3} \mu \delta_{i j} \frac{\partial u_{k}}{\partial x_{k}}+\mu\left(\frac{\partial u_{i}}{\partial x_{j}}+\frac{\partial u_{j}}{\partial x_{i}}\right)
$$

where $\mu$ denotes the coefficient of viscosity (dynamic viscosity, molecular viscosity).

Sometimes, the mass balance equation and the momentum balance equation together are called as the Navier-Stokes equations. 


\subsubsection{Energy Balance Equation}

The conservation of energy follows from the first law of thermodynamics. Applying it to a fluid passing through an infinitesimal fixed control volume, we can get the energy balance equation in terms of the specific total energy $E$,

$$
\frac{\partial \rho E}{\partial t}+\frac{\partial\left(\rho E u_{i}\right)}{\partial x_{i}}=-\frac{\partial q_{j}}{\partial x_{j}}+\rho g_{i} u_{i}-\frac{\partial}{\partial x_{j}}\left(p u_{j}\right)+\frac{\partial}{\partial x_{i}}\left(\tau_{i j} u_{j}\right),
$$

where $E$ denotes the total energy per unit volume, $q_{i}$ is the $i^{\text {th }}$ component of the heat flux vector q.

Similar to the momentum balance equation, the left-hand side of Eq. (2.6) is also composed by a time derivative and a convection derivative of the energy. In the right-hand side of the energy balance equation, the first term represents the rate change of heat transfer by conduction through the control surface, the second term represents the work done by the body forces, and the other terms represent the work done by the surface forces per unit volume.

\subsection{Introduction to turbulence}

The ubiquity of turbulent flow has made it an important object of study for mathematicians, physicists and engineers for more than two centuries. Turbulent flows play an important role in many scientific fields such as aerodynamics, chemistry and engineering. Turbulence 
occurs in many flows of practical interest and may strongly enhance the particle dispersion and the heat transfer of these flows. ${ }^{3}$

It is hard to give an exact definition of turbulent flows. In 1975, Hinze ${ }^{11}$ gave the following definition of turbulence: "turbulent fluid motion is an irregular condition of flow in which the various quantities show a random variation with time and space coordinates so that statistically distinct average values can be discerned."

Smoke rising from a cigarette is a good example of turbulent flow. The smoke moves straight up for the first few centimeters, and then becomes unstable and diluted. At last, it disappears in the air, or more exactly, mixes with the atmosphere. When smoke moves straight up, it was laminar, then it turned turbulent. This example illustrates the process of how laminar flow turns into turbulent flow. ${ }^{8}$

The Reynolds number, Re, is a simple criterion to determine whether the flow is laminar, transient or turbulent. The Reynolds number is defined as

$$
\operatorname{Re} \equiv \frac{\rho U L}{\mu}=\frac{U L}{v},
$$

where $v=\mu / \rho$ represents the kinematic fluid viscosity, $U$ and $L$ denote the characteristic velocity and length, respectively. For a pipe flow, increasing the inflow velocity, i.e. the characteristic velocity, leads to the transition of the flow from laminar $(\operatorname{Re}<2300)$, transient $(2300 \leq R e \leq 4000)$ to turbulent $(\operatorname{Re}>4000) .{ }^{12}$ 
Turbulence is based on Kolmogorov's theory ${ }^{13}$ of self similarity: "large eddies of the flow are dependent on the flow geometry, while smaller eddies are self similar and have a universal character". More precisely, the large eddies are usually vortical, but their shapes and strengths are flow dependent and highly anisotropic. However, the small eddies are nearly isotropic and hence much easier to model. The large eddies have the large velocity fluctuations and diffuse most of the mass, momentum and energy. They interact not only with the mean flow but also with each other. The nonlinear interactions between the large eddies generate the small eddies which dissipate into heat eventually.

The Kolmogorov-Richardson energy cascade theory points out that the turbulence kinetic energy flows from the largest scale to the smallest scale, the Kolmogorov length scale $\eta$, where the viscous dissipation occurs. ${ }^{14}$ The Kolmogorov length scale $\eta$ is defined as

$$
\eta \equiv\left(\frac{v^{3}}{\varepsilon}\right)^{1 / 4}
$$

where the dissipation rate $\varepsilon$ is the average rate of turbulent energy dissipation by molecular viscosity per unit mass. Note that the accompanied Kolmogorov time scale, $\tau_{\eta}$, is given by

$$
\tau_{\eta}=\left(\frac{v}{\varepsilon}\right)^{1 / 2}
$$




\subsubsection{The physical nature of turbulence}

Turbulent flows have the following physical features ( for details refer to Refs. ${ }^{15-17}$ ):

- High Reynolds numbers. Turbulence arises at high Reynolds numbers.

- Continuity. Turbulence is a continuum phenomenon, and it can be described by a continuum approximation, such as the Navier-Stokes equations.

- Random. Turbulence is a random process. It is irregular and unpredictable in detail. Nonetheless, the average statistical properties of turbulent flows are reproducible.

- Multi-Scales. The largest length scale of a turbulent flow is called the integral length scale $L$, which is determined by the geometry of the flow, and the corresponded integral time scale is defined as $\tau_{L}=L^{2} / v$. From the largest eddies with the integral length scale $L$,

the turbulence energy cascade generates smaller and smaller scales. The energy cascade is terminated by viscosity, and the relative smallest scale is the Kolmogorov length scale $\eta$.

- Dissipative. Turbulence dissipates energy from large eddies to small eddies via the turbulence energy cascade.

- High Diffusivity In comparison to laminar flows, turbulent flows greatly enhance the rate of transport and mixing of mass, momentum and energy. This is extremely 
important in many applications including fuel spray processes, which are of prime interest in this dissertation.

- Small-scale random vorticity. Turbulent flows have intense, small-scale vorticities. They are random in both, space and time.

\subsubsection{Overview of turbulence solution approaches}

Adiabatic turbulent flows are also governed by the Navier-Stokes equations. To solve the Navier-Stokes equations, the analytical solution methods can only be applied to simplified flow problems. Technically relevant flows require numerical solution methods. Most numerical solution strategies require the discretisation of space and time. The physical space is discretised into a great quantity of cells. This process is referred as mesh generation. Three of the most popular turbulence modeling approaches are direct numerical simulation (DNS), Reynolds-averaged Navier-Stokes method (RANS) and large eddy simulation (LES).

\subsubsection{Direct numerical simulation}

Direct numerical simulations solve all the scales of turbulence ranged from the smallest dissipative Kolmogorov scales to the integral scales. 
For example, in turbulent channel flow, the turbulent Reynolds number $R e_{t}$ is defined as

$$
R e_{t} \equiv \frac{u_{t} L}{v},
$$

where $u_{t}$ denotes the turbulent wall-shear velocity. To satisfy the resolution requirements, the number $\mathrm{N}$ of mesh points with increments $\mathrm{h}$ along a given direction, must satisfy

$$
N \cdot h \geq L \text { and } h \leq \eta \text {. }
$$

So that both the integral scale $L$ and the Kolmogorov scale $\eta$ can be resolved in the computation domain. The relations in Eq. (2.11) imply that $N \geq L / \eta$. Replacing the Kolmogorov length scale $\eta$ by its definition in Eq. (2.8), and eliminating $v$ by Eq. (2.10), we have

$$
N \geq \operatorname{Re}_{t}^{\frac{3}{4}}\left(\frac{L \varepsilon}{u_{t}^{3}}\right)^{\frac{1}{4}}
$$

Since by Ref. ${ }^{18}$

$$
\varepsilon \approx \frac{u_{t}^{3}}{L}
$$

we can conclude that

$$
N \geq R e_{t}^{\frac{3}{4}} .
$$

It follows from Eq. (2.14), that the computational effort and the memory storage requirements in DNS increases very fast with the turbulent Reynolds number $R e_{t}$. Limited by the computation capacities of today's computers, DNS can only solve the simple geometry 
turbulence problems or turbulent flows at low Reynolds number. Based on this analysis, it is apparent that spatial and temporal resolutions have to be relaxed, which, as discussed next, motivates the use of RANS and LES (See Fig. 2.1).

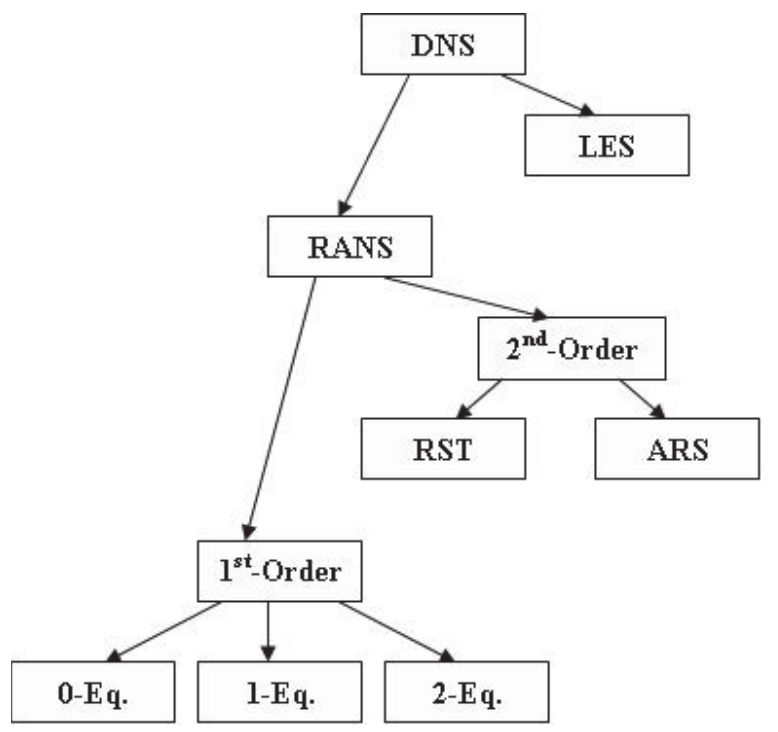

Figure 2.1: Turbulence models ordered according to their decreasing level of complexity. Abbreviations: RST = Reynolds-Stress Transport models; ARS = Algebraic Reynolds-Stress models.

\subsubsection{Reynolds-averaged Navier-Stokes method}

In this approach, turbulence is viewed as a statistical phenomenon. As explained later, RANS solve the time-averaged Navier-Stokes equations and give statistically averaged solutions. The averaged flow variables can reflect the main properties of the turbulent flow. Therefore, RANS can provide detailed localized descriptions of the flow and turbulence fields, including profiles of the streamwise velocity and the Reynolds shear stress. Thus, RANS has been applied widely in engineering problems. 
However, the time-averaging of the NS equations generates an unknown term, the Reynolds stress tensor. This term leads to the notorious closure problem, namely, that there are more unknowns than equations. Consequently, turbulence models are needed to account for those unknowns.

For unsteady flows, the accuracy of the RANS method using $1^{\text {st }}$ order closure models is not guaranteed. Moreover, these models cannot provide instantaneous information of turbulent

flows. However, the $2^{\text {nd }}$ order closure models, such as the Reynolds-stress transport model implemented by Yang et al., ${ }^{19}$ give much better results. The draw back of second order closure models is that they are much more challenging to implement.

\subsubsection{Large eddy simulation}

In large eddy simulation (LES), the large scale motions are calculated by solving the spatial-filtered NS equations, while the small scale motions are modeled by the sub-grid scale (SGS) stress models.

The computation time required for LES is less than DNS but greater than RANS. However, RANS can only produce "time-averaged" results, while LES is able to predict more details of the instantaneous flows. In chemical reactions, the time-averaged species equations may fail to produce the high enough species concentrations such that the chemical reactions can occur. Additionally, in the simulations of flow separation, LES also provides more details of the flow motions over RANS. ${ }^{1,20}$ 


\subsection{Reynolds-averaged Navier-Stokes method}

\subsubsection{Time averaging}

The random feature of turbulent flow suggests that an instantaneous flow variable $f^{o}(\mathbf{x}, t)$ can be decomposed to a time-averaged part $f(\mathbf{x}, t)$ and a fluctuation part $f^{\prime}(\mathbf{x}, t)$,

$$
f^{o}(\mathbf{x}, t)=f(\mathbf{x}, t)+f^{\prime}(\mathbf{x}, t)
$$

Then one only needs to solve the governing equations for the mean values. Note that the type of time-averaging (Reynolds or Favre, as discussed below) will give different values for the averaged and fluctuation terms. For convenience, the same notations, $f$ and $f^{\prime}$, are used for different type of time-averaging in this thesis. However, it is usually clear from the context which type is used.

\subsubsection{Reynolds time averaging}

In 1985, Reynolds time averaging is introduced by Reynolds as the first method to solve the turbulent flows approximately. ${ }^{21}$ Considering the turbulent flow as statistically stationary flow, the Reynolds time average of the flow variable $f^{o}(\mathbf{x}, t)$ over a time interval $T$ is defined by

$$
f(\mathbf{x}, t)=\overline{f^{o}}(\mathbf{x}, t) \equiv \frac{1}{T} \int_{t}^{t+T} f^{o}(\mathbf{x}, s) d s
$$


Reynolds averaging satisfies the following properties, for any two instantaneous flow variables $f^{o}, g^{o}$ and the independent variables,

$$
\overline{\overline{f^{o}} g^{o}}=\overline{f^{o}} \overline{g^{o}}, \overline{f^{\prime}}=0, \overline{\overline{f^{o}}}=\overline{f^{o}}, \overline{f^{o}+g^{o}}=\overline{f^{o}}+\overline{g^{o}}, \frac{\overline{\partial f^{o}}}{\partial s}=\frac{\partial \overline{f^{o}}}{\partial s}
$$

\subsubsection{Favre time averaging}

For compressible flows, the density is not constant, then Reynolds time averaging leads to additional density fluctuation terms which make the averaged governing equations more complicated. Hence, it is more convenient to apply the Favre time averaging (density weighted time averaging) on the flow variables. The Favre time average of the flow variable $f^{o}(\mathbf{x}, t)$ over a time interval $T$ is defined as,

$$
f(\mathbf{x}, t)=\widetilde{f^{o}}(\mathbf{x}, t) \equiv \frac{\int_{t}^{t+T} \rho^{o}(\mathbf{x}, s) f^{o}(\mathbf{x}, s) d s}{\int_{t}^{t+T} \rho^{o}(\mathbf{x}, s) d s} .
$$

Note that in accordance with Eq. (2.15), here $f(\mathbf{x}, t)$ denotes Favre-averaging and $f\left({ }^{\prime} x, t\right)$ denotes the Favre fluctuations. The Favre averaging and Reynolds averaging have the following relationships:

$$
\overline{\rho^{o} f^{o}}=\overline{\rho^{o} \widetilde{f^{o}}}=\overline{\rho^{o}} \widetilde{f^{o}}, \widetilde{\rho^{o} f^{\prime}}=0, \text { but } \widetilde{f}^{\prime} \neq 0
$$




\subsubsection{Time-averaged Navier-Stokes equations}

Applying the Favre decomposition to velocity, the Reynolds decomposition to the remaining flow variables, and then the Reynolds time averaging to Eq. (2.1) and Eq. (2.4) yield the time-averaged Navier-Stokes equations:

$$
\begin{gathered}
\frac{\partial \rho}{\partial t}+\frac{\partial}{\partial x_{j}}\left(\rho u_{j}\right)=0, \\
\frac{\partial}{\partial t}\left(\rho u_{i}\right)+\frac{\partial}{\partial x_{j}}\left(\rho u_{j} u_{i}\right)=-\frac{\partial P}{\partial x_{i}}+\frac{\partial}{\partial x_{j}}\left(\tau_{i j}+\tau_{i j}^{R}\right),
\end{gathered}
$$

where $u_{i}=\widetilde{u_{i}^{o}}$ is the Favre averaged velocity, $\rho=\overline{\rho^{o}}, P=\overline{P^{o}}$ and $\tau_{i j}=\overline{\tau_{i j}^{o}}$ are all Reynolds averaged. The new variable, Reynolds stress tensor $\tau_{i j}^{R}$, is defined as ${ }^{22}$

$$
\tau_{i j}^{R}=-\widetilde{\rho u_{i}^{\prime} u_{j}^{\prime}}
$$

The Reynolds stress tensor measures the effects of the turbulent fluctuations to the the mean flow dynamics, and it can not be formulated explicitly without resolving the smallest scales of the flow. ${ }^{22}$ Then the number of unknowns is greater than the number of the equations. This is referred as the closure problem, which will be discussed next in more details. 


\subsubsection{RANS closure problem}

By the above discussion, the Reynolds or Favre time-averaging of Navier-Stokes equations yield an new term, Reynolds-stress tensor $\tau^{R}$. The Reynolds-stress tensors are associated with the turbulent motions. To balance the number of unknowns and the number of equations, further assumptions and approximations about the new quantities lead to the "closure" of the system.

According to the Boussinesq assumption, ${ }^{23}$ the Reynolds-stress $\tau^{R}$ is assumed to linearly relate to the the mean flow straining field as following

$$
\tau_{i j}^{R}=2 \mu_{t} S_{i j}-\frac{2}{3} \rho k \delta_{i j}
$$

where the mean turbulent kinetic energy $k \equiv \frac{1}{2} \widetilde{u_{j}^{\prime} u_{j}^{\prime}}, \mu_{t}$ denotes the turbulent viscosity, and the mean strain rate is defined by

$$
S_{i j} \equiv \frac{1}{2}\left(\frac{\partial u_{i}}{\partial x_{j}}+\frac{\partial u_{j}}{\partial x_{i}}\right)-\frac{1}{3} \frac{\partial u_{k}}{\partial x_{k}} \delta_{i j}
$$

\subsubsection{Standard $k-\varepsilon$ model}

The standard $k-\varepsilon$ model $^{4,24,25}$ is one of the most popular RANS closure models. It assumes the turbulent viscosity to be dependent on two transport variables, the turbulent 
kinetic energy $k$ and the dissipation rate $\varepsilon$, and is given by

$$
\mu_{t}=C_{\mu} \rho k^{2} / \varepsilon,
$$

where $C_{\mu}=0.09$.

A flow can be divided into a mean part and a turbulent part. The kinetic energy of the turbulent part is measured by the turbulent kinetic energy $k,{ }^{16}$ which is given by

$$
k=\frac{1}{2}\left\|\mathbf{u}^{\prime}\right\|^{2} .
$$

The dissipation rate $\varepsilon$ is defined as the rate of conversion of turbulence into heat by molecular viscosity, ${ }^{26}$

$$
\varepsilon \equiv 2 \vee \widetilde{S_{i j} S_{i j}}
$$

The following two transport equations are used to close the two new transport variables $k$ and $\varepsilon$ :

$$
\begin{gathered}
\frac{\partial \rho k}{\partial t}+\frac{\partial \rho k u_{i}}{\partial x_{i}}=\frac{\partial}{\partial x_{j}}\left[\left(\alpha_{k} \mu+\mu_{t}\right) \frac{\partial k}{\partial x_{j}}\right]+P_{k}-\frac{2}{3} \rho k \frac{\partial u_{k}}{\partial x_{k}}-\rho \varepsilon \\
\frac{\partial \rho \varepsilon}{\partial t}+\frac{\partial \rho \varepsilon u_{i}}{\partial x_{i}}=\frac{\partial}{\partial x_{j}}\left[\left(\alpha_{\varepsilon} \mu+\mu_{t}\right) \frac{\partial \varepsilon}{\partial x_{j}}\right]+C_{1} P_{k} \frac{\varepsilon}{k}-\left(\frac{2}{3} C_{1}+C_{3}\right) \rho \varepsilon \frac{\partial u_{k}}{\partial x_{k}}-C_{2} \rho \frac{\varepsilon^{2}}{k}
\end{gathered}
$$

where $C_{1}=1.44, C_{2}=1.92, C_{3}=-0.33, \alpha_{k}=1.0, \alpha_{\varepsilon}=0.76923$ and the production term $P_{k}$ is defined as $P_{k}=\mu_{t}|S|^{2}$, where $|S| \equiv \sqrt{2 S_{i j} S_{i j}}$. 


\subsubsection{RNG $k-\varepsilon$ model}

In the standard $k-\varepsilon$ model, the Reynolds stress is described by low-order expansions, and the diffusion production term only accounts for specified scales of motion. ${ }^{5}$ This motivated the development of the Re-Normalisation Group (RNG) approach, where a modified $\varepsilon$ equation together with the original $k$ equation are called the $\mathrm{RNG} k-\varepsilon$ equations,

$$
\begin{gathered}
\frac{\partial \rho k}{\partial t}+\frac{\partial \rho k u_{i}}{\partial x_{i}}=\frac{\partial}{\partial x_{j}}\left[\left(\alpha_{k} \mu+\mu_{t}\right) \frac{\partial k}{\partial x_{j}}\right]+P_{k}-\frac{2}{3} \rho k \frac{\partial u_{k}}{\partial x_{k}}-\rho \varepsilon \\
\frac{\partial \rho \varepsilon}{\partial t}+\frac{\partial \rho \varepsilon u_{i}}{\partial x_{i}}=\frac{\partial}{\partial x_{j}}\left[\left(\alpha_{\varepsilon} \mu+\mu_{t}\right) \frac{\partial \varepsilon}{\partial x_{j}}\right]+\left(C_{1}-R\right) P_{k} \frac{\varepsilon}{k}-\left(\frac{2}{3} C_{1}+C_{3}\right) \rho \varepsilon \frac{\partial u_{k}}{\partial x_{k}}-C_{2} \rho \frac{\varepsilon^{2}}{k}
\end{gathered}
$$

where $C_{1}=1.42, C_{2}=1.68, C_{3}=-0.33, \alpha_{k}=1.39, \alpha_{\varepsilon}=1.39$, and $R=\frac{\eta\left(-\eta / \eta_{0}+1\right)}{\beta \eta^{3}+1}$ with $\eta=|S| k / \varepsilon, \eta_{0}=4.38$ and $\beta=0.012$. The constant $C_{\mu}$ in Eq. (2.22) for RNG $k-\varepsilon$ equations equals 0.0845 .

\subsection{Large eddy simulation}

In DNS, the flow motions have to be resolved to the Kolmogorov length scale $\eta$ which requires high computation costs. To resolve the fluid flow on a relatively coarse grid, LES performed a low-pass spatial filtering over the control volume. The large turbulence structures are resolved by the spatial-filtered Navier-Stokes equations in LES. The small eddies are modeled by the subgrid scale models. 


\subsubsection{Spatial filtering}

Spatial filtering decomposes any instantaneous flow variable $f^{o}(\mathbf{x}, t)$ into a filtered part $f(\mathbf{x}, t)$ and a fluctuation part $f^{\prime}(\mathbf{x}, t)$, i.e.,

$$
f^{o}(\mathbf{x}, t)=f(\mathbf{x}, t)+f^{\prime}(\mathbf{x}, t)
$$

Here, the filtered part is given by

$$
f\left(\mathbf{x}_{0}, t\right)=\overline{f^{o}}\left(\mathbf{x}_{0}, t\right)=\int_{\Omega} f^{o}(\mathbf{x}, t) G\left(\mathbf{x}_{0}, \mathbf{x}, \triangle\right) d \mathbf{x}
$$

where $\mathbf{x}_{0}$ is any position vector in the computation domain $\Omega, G$ is the filter function and $\Delta=\left(\Delta_{1}, \Delta_{2}, \Delta_{3}\right)^{1 / 3}$ is the filter width. ${ }^{8}$ The filter size is usually half of the grid size in order to resolve as many turbulence scales as possible. ${ }^{3}$

The three widely used filter functions, the tophat filter $G_{1}$, the sharp Fourier cut-off filter $G_{2}$ and the Gaussian filter $G_{3}$ are defined as. ${ }^{8}$

$$
\begin{gathered}
G_{1}\left(\mathbf{x}_{0}, \mathbf{x}, t\right)= \begin{cases}1 / \Delta^{3}, & \text { if }\left|\left(x_{0}\right)_{i}-x_{i}\right| \leq \Delta_{i} / 2 \\
0, & \text { otherwise. }\end{cases} \\
G_{2}\left(\mathbf{x}_{0}, \mathbf{x}, t\right)=\prod_{i=1}^{3} \frac{\sin \left(\frac{\pi}{\Delta_{i}}\left[\left(x_{0}\right)_{i}-x_{i}\right]\right)}{\pi\left[\left(x_{0}\right)_{i}-x_{i}\right]} \\
G_{3}\left(\mathbf{x}_{0}, \mathbf{x}, t\right)=\left(\frac{6}{\pi \Delta^{2}}\right)^{3 / 2} \exp \left(\frac{-6|| \mathbf{x}_{0}-\mathbf{x} \|_{2}^{2}}{\Delta^{2}}\right)
\end{gathered}
$$


For further manipulation of the Navier-Stokes equations, all these filters are required to satisfy the following three fundamental properties: ${ }^{27}$

- Consistency

$$
\int_{\Omega} G\left(\mathbf{x}_{0}, \mathbf{x}, t\right) d \mathbf{x}=1
$$

- Linearity

$$
\overline{f^{o}+g^{o}}=\overline{f^{o}}+\overline{g^{o}}
$$

- Commutation with differentiation

$$
\frac{\overline{\partial f^{o}}}{\partial s}=\frac{\partial \overline{f^{o}}}{\partial s}, s=x_{i}, t
$$

In contrast to the Reynolds time averaging, however, we have

$$
\overline{\overline{f^{o}}} \neq \overline{f^{o}} \text { and } \overline{f^{\prime}} \neq 0
$$

In compressible flow, the term $\rho u_{j}$ in the continuity equation can be decomposed as

$$
\overline{\rho^{o} u_{j}^{o}}=\overline{\rho^{o}\left(u_{j}+u_{j}^{\prime}\right)} .
$$

Since the fluctuation term cannot be eliminated as in Reynolds time averaging, the spatial filtering decomposition of velocity in the above equation generates an extra subgrid term. 
This can be avoid by the change of variable, ${ }^{27}$

$$
\overline{\rho^{o} f^{o}}=\overline{\rho^{o}} \widetilde{f^{o}}
$$

where the $(\tilde{*})$ operator refers to the Favre averaging. As the result, the term $\overline{\rho^{o} u_{j}^{o}}$ can be transformed to $\overline{\rho^{o}} \widetilde{u_{j}^{o}}$.

The Favre-averaged spatial-filtered variable is defined as

$$
f\left(\mathbf{x}_{0}, t\right)=\widetilde{f}^{o}\left(\mathbf{x}_{0}, t\right) \equiv \frac{\int_{\Omega} \rho^{o}(\mathbf{x}, t) f^{o}(\mathbf{x}, t) G\left(\mathbf{x}_{0}, \mathbf{x}, \triangle\right) d \mathbf{x}}{\int_{\Omega} \rho^{o}(\mathbf{x}, t) G\left(\mathbf{x}_{0}, \mathbf{x}, \triangle\right) d \mathbf{x}}
$$

The $(\tilde{*})$ operator is linear but does not commute with differentiation

$$
\widetilde{\frac{\partial f^{o}}{\partial s}} \neq \frac{\partial \widetilde{f^{o}}}{\partial s}, s=x_{i}, t
$$

For any fluctuation term $f^{\prime}$ which is generated by Favre-averaged spatial filtering, one has

$$
\overline{\rho^{o} f^{\prime}}=0 \text {. }
$$

It leads to the change of variable transformation in Eq. (2.33). 


\subsubsection{Spatial-filtered Navier-Stokes equations}

Using the Favre spatial filtering, spatial-filtered Navier-Stokes equations are extracted from Eq. (2.1) and Eq. (2.4) as

$$
\begin{gathered}
\frac{\partial \rho}{\partial t}+\frac{\partial}{\partial x_{j}}\left(\rho u_{j}\right)=0 \\
\frac{\partial}{\partial t}\left(\rho u_{i}\right)+\frac{\partial}{\partial x_{j}}\left(\rho u_{j} u_{i}\right)=-\frac{\partial P}{\partial x_{i}}+\frac{\partial}{\partial x_{j}}\left(\tau_{i j}+\tau_{i j}^{S}\right),
\end{gathered}
$$

where $u_{i}$ is the Favre-averaged spatial-filtered velocity, and all the other variables are all spatial-filtered. The subgrid-scale stress is defined as,

$$
\tau_{i j}^{S}=-\rho\left(\widetilde{u_{i}^{o} u_{j}^{o}}-\widetilde{u^{o}}{ }_{i}{\widetilde{u_{j}^{o}}}^{o} .\right.
$$

The subgrid-scale stress tensor $\tau_{i j}^{S}$ generated by spatial filtering is analogous to the Reynolds-stress tensor $\tau_{i j}^{R}$ in Eq. (2.20).

\subsubsection{Subgrid-scale closure models}

In LES, only the spatial-filtered Navier-Stokes equations for the evolution of the large eddies are resolved. The effect of the small-scale eddies below a certain size, the subgrid size, are neglected. To correct the lack of small-scales in the filtered equations, the small eddies are described by the subgrid-scale (SGS) models. This process is known as the subgrid-scale (SGS) modeling. 
The SGS models mimic the interactions between those scales that are filtered (small-scale) and those which are resolved (large-scale). Here, the Smagorinsky model ${ }^{2}$ and the oneequation model ${ }^{7,28,29}$ will be introduced.

\subsubsection{Smagorinsky model}

In the Smagorinsky model, the small scale eddies are assumed to dissipate instantaneously all the energy transferred from the large eddies. ${ }^{8}$

Similar to the eddy viscosity modeling of the Reynolds-stress for RANS in Eq. (2.20), the residual stress is given by

$$
\tau_{i j}^{S}=2 \mu_{t} S_{i j}
$$

where the turbulent kinematic viscosity $\mu_{t}$ is modeled to be proportional to the magnitude of the mean strain rate,

$$
\mu_{t}=\rho\left(C_{s} \Delta\right)^{2}|\tilde{S}|
$$

with the Smagorinsky constant $C_{S}$ in the range of 0.1 to 0.2 , and $\Delta$ denotes the filter width. 


\subsubsection{LES one-equation model}

In the one equation model, the residual stress tensor is modeled as following

$$
\tau_{i j}^{S}=2 \mu_{t} S_{i j}-\frac{2}{3} \rho k^{s g s} \delta_{i j}
$$

where the subgrid-scale turbulent kinetic energy $k^{s g s}$ is defined by 29,30

$$
k^{s g s} \equiv\left(\widetilde{u_{k}^{o} u_{k}^{o}}-\widetilde{u^{o}}{\widetilde{u^{o}}}_{k}\right) / 2
$$

The turbulent viscosity is modeled by

$$
\mu_{t}=C_{\mu} \rho \Delta \sqrt{k^{s g s}}
$$

where $\Delta$ denotes the characteristic grid length scale, and $C_{k}$ is an adjustable model parameter. The value of $C_{\mu}$ depends on the type of flow, eg., shear flow, free flow, swirl flow, etc. ${ }^{31}$ The default value for $C_{\mu}$ is 0.067 .

The subgrid kinetic energy $k^{s g s}$ is the new unknown to be closed by the $k^{s g s}$ equation,

$$
\frac{\partial \rho k^{s g s}}{\partial t}+\frac{\partial}{\partial x_{i}}\left(\rho u_{i} k^{s g s}\right)=\frac{\partial}{\partial x_{i}}\left(\frac{\mu_{t}}{P_{r_{t}}} \frac{\partial k^{s g s}}{\partial x_{i}}\right)-\tau_{i j}^{S} \frac{\partial u_{j}}{\partial x_{i}}-C_{\varepsilon} \rho k^{s g s 3 / 2} / \Delta
$$

with $C_{\varepsilon}=0.916$ 


\subsection{Computational Aspects}

The real flow is mathematically modeled by several governing differential equations which have been discussed in Sections 2.1-2.4. Then, the differential equations are discretized and solved numerically in a segregated manner. The discretization algorithms will be introduced in Section 2.5.2.

The segregated solution procedure is based on the following steps:

1. The fluid pressure value is updated by renewing the temperature value at the time step " $n+1$ " in the equation of state. For any scalar variable $\phi$, the face flux value $\bar{\phi}_{f}$ in Eq. (2.50) at time step " $\mathrm{n}+1$ " is calculated using the cell center value of $\phi$ at time step "n".

2. The momentum balance equations are solved with the above pressure field.

3. The resolved velocity field in the previous step may not necessarily satisfy the continuity equation. Thus a pressure correction equation based on the continuity equation is used to correct the pressure, the velocity fields and the density. This step is usually referred as the pressure-velocity coupling. A detailed introduction will be given in Section 2.5.3.

4. The heat equation is solved using the values of pressure and velocity obtained in the previous steps. 
5. Check the convergence of the solution. If the convergence is not obtained, restart from step 1. Otherwise, progress to the next time step.

\subsubsection{Computation code}

This thesis uses an user-defined code based upon OpenFOAM-1.5, ${ }^{32-34}$ an open-source object-oriented $\mathrm{C}++$ code. OpenFOAM is chosen for its convenience in developing new models and its intrinsic architecture for parallelization.

In OpenFOAM, the conservation equations are discretized in time and space and solved using the finite volume method (FVM). ${ }^{32,35}$ The FVM in OpenFOAM integrated the differential equations over each cell of the polyhedral mesh. ${ }^{34}$ All fluid dynamic properties are stored at the control volume centroid. Details of the meshes will be discussed in Chapter 3 and Chapter 4. The discretized equation for each variable is solved iteratively. The convergence error tolerance is $10^{-7}$ for pressure and $10^{-6}$ for the remaining variables.

Time step will be adjusted such that the whole solution procedure is not only stable but also computationally efficient. The variable time step is achieved by controlling the Courant number, which is defined as,

$$
C o=\frac{U \Delta t}{\Delta x}
$$

where $U$ is the magnitude of the flow velocity, $\Delta t$ is the current time step and $\Delta x$ the 
distance between adjacent cell centers. The higher the Courant number, the larger the timestep for a fixed mesh. According to the Courant-Friedrichs-Lewy (CFL) condition, ${ }^{36}$ the Courant number should be less than 1 to ensure the stability in the explicit time schemes. Defining the maximum value of the Courant number in the file $\$$ case/system/controlDict as $\max C o=0.1$, the time step will be adjusted automatically in OpenFOAM. Increasing the maximum Courant number accelerates the solution procedure, but the system may become unstable. The user can tune the maximum Courant number by observing the error variations.

Table 2.1

The values of $\phi$ and $\Gamma$ in the Navier-Stokes equations

\begin{tabular}{ccc}
\hline NS Eqn. & $\phi$ & $\Gamma$ \\
\hline Mass balance Eq. (2.1) & 1 & 0 \\
Momentum balance Eq. (2.2) & $u_{i}$ & $\mu$ \\
Energy balance Eq. (2.6) & $E$ & 0 \\
\hline
\end{tabular}

\subsubsection{Discretization}

The Navier-Stokes Eqs. (2.1)-(2.6) describe the transport of mass, momentum and heat. They can be summarized by the general form of the transport equations, i.e. the generic transport equation for a scalar intensive property $\phi:^{37}$

$$
\underbrace{\frac{\partial \rho \phi}{\partial t}}_{\text {Transient }}+\underbrace{\nabla \cdot(\rho \mathbf{u} \phi)}_{\text {Convection }}=\underbrace{\nabla \cdot(\Gamma \nabla \phi)}_{\text {Diffusion }}+\underbrace{S_{\phi}}_{\text {Source }}
$$


where $\Gamma$ is the the diffusion coefficient. The values of $\phi$ and $\Gamma$ in the corresponding NavierStokes equations are shown in Table 2.1. The four terms in Eq. (2.44) are explained as follows:

The transient term, i.e. the time derivative term, accounts for the accumulation of $\phi$ during each time step;

The convection term accounts for the transport of $\phi$ due to the velocity field $\mathbf{u}$;

The diffusion term accounts for the transport of $\phi$ due to its gradients;

The source term, accounts for the spray sources which influence $\phi$.

In this study, the transport equation is discretized using the finite volume method (FVM). ${ }^{32}$ It takes the integration of the governing equations on each control volume, and the derivative terms are converted into the surface integrals using the divergence theorem. ${ }^{10}$ The integration of the generic transport equation, i.e. Eq. (2.44), over an arbitrary control volume $V$ is shown as follows,

$$
\int \frac{\partial \rho \phi}{\partial t} d V+\oint \rho \mathbf{u} \phi \cdot d \mathbf{A}=\oint \Gamma \nabla \phi \cdot d \mathbf{A}+\int S_{\phi} d V
$$

where A denotes the surface area vector. 
Applying the integral transport equation (Eq. (2.45)) to all computation cells, the generic transport equations can be discretized as,

$$
\frac{\partial \rho \phi}{\partial t} V+\sum_{f}^{N_{f}} \rho_{f} \phi_{f} \mathbf{u}_{f} \cdot d \mathbf{A}_{f}=\sum_{f}^{N_{f}} \Gamma_{f}(\nabla \phi)_{n} \cdot d \mathbf{A}_{f}+S_{\phi} V
$$

where $N_{f}$ counts the number of cell faces, $(\nabla \phi)_{n}$ is the projection of the vector $\nabla \phi$ on the normal direction of the face $f$, and the subscript $(\cdot)_{f}$ represents the corresponding value convected through the face $f$.

The time derivative term is discretized using Euler's implicit first order time scheme,

$$
\frac{\partial \rho \phi}{\partial t}=\frac{\phi^{n+1}-\phi^{n}}{\Delta t}
$$

where $\Delta t$ is the time step, $\phi^{n+1}$ and $\phi$ are the values of $\phi$ at time step "n+1" and "n".

The face values $\phi_{f}$ at time step " $n+1 "$ are predicted by the second order upwind scheme based on the cell center values at time step "n", ${ }^{32}$

$$
\phi_{f}=\phi+\nabla \phi \cdot \mathbf{r}
$$

where vector $\mathbf{r}$ is the displacement from the cell center to the face center point. 
The gradient term $\nabla \phi$ is evaluated using the Green-Gauss Theorem, ${ }^{38}$

$$
\nabla \phi=\frac{1}{V} \sum_{f} \bar{\phi}_{f} \mathbf{A}_{f}
$$

where the face value $\bar{\phi}_{f}$ is the average of the values of $\phi$ at the cell centers of the two neighboring computation cells,

$$
\bar{\phi}_{f}=\frac{\phi_{c e l l 0}+\phi_{c e l l 1}}{2}
$$

\subsubsection{Pressure-velocity coupling}

The aim of pressure velocity coupling techniques is to ensure that the resolved pressure and velocity satisfy both the momentum and the continuity equation. For this aim, the continuity equation is reformed to introduce an additional condition for pressure as shown in Eq. (2.56), i.e. the pressure correction equation. SIMPLE $^{39}$ (Semi-Implicit Method for Pressure-Linked Equations) and PISO ${ }^{40}$ (Pressure-Implicit with Splitting of Operators) are both widely used pressure-based segregated algorithms. In this work, the coupling between the velocity equations and the pressure equation is performed using the SIMPLE algorithm for the steady-state flows and the PISO algorithm for the transcient flows. For more details on the SIMPLE and PISO algorithms refer to Ref. ${ }^{41}$ 


\subsubsection{SIMPLE}

For steady-flows, the integral form of the continuity and momentum equations can be written as:

$$
\begin{aligned}
\oint \rho \mathbf{u} \cdot d \mathbf{A} & =0 \\
\oint \rho \mathbf{u u} \cdot d \mathbf{A} & =-\oint p \mathbf{I} \cdot d \mathbf{A}+\oint \tau \cdot d \mathbf{A}+\int_{V} \rho \mathbf{g} d V
\end{aligned}
$$

where $\mathbf{I}$ is the identity matrix.

The momentum equation can be discretized similar to the generic transport equation as in Eq. (2.46). Substitute the scalar $\phi$ by the velocity component $u_{1}$, the x-momentum equation can be discretized and then linearized as, ${ }^{38}$

$$
a_{P} u_{1}=\sum_{n b} a_{n b} u_{1, n b}+\sum_{f} p_{f} \mathbf{A}_{f} \cdot \hat{i}+S
$$

where the subscript $(\cdot)_{n b}$ represents the neighboring cell, $a_{P}$ and $a_{n b}$ are the linearized coefficients for $u_{1}$ and $u_{1, n b}, p_{f}$ is the pressure at the face $f$, and $S$ refers to source term. The face value of the pressure, $p_{f}$, can be weighted averaged by the centroid values of pressures within the two neighboring cells $c 0$ and $c 1$ of the face $f$,

$$
p_{f}=\frac{\frac{p_{c 0}}{a_{P, c 0}}+\frac{p_{c 1}}{a_{P, c 1}}}{\frac{1}{a_{P, c 0}}+\frac{1}{a_{P, c 1}}},
$$


where the weighting factors $a_{P, c 0}$ and $a_{P, c 1}$ are the linearized coefficients in the relative linearized momentum Eq. (2.53) for the two neighboring cells.

The integral form of the continuity equation (Eq. (2.51)) can also be discretized as follows,

$$
\sum_{f}^{N_{\text {faces }}} J_{f} A_{f}=0
$$

where $J_{f}$ is the mass flux through the face $f$. To avoid the unphysical checker-boarding of pressure, ${ }^{42} J_{f}$ is not averaged linearly as in Eq. (2.50), but calculated under a momentum weighted averaging scheme, ${ }^{43}$

$$
J_{f}=\hat{J}_{f}+d_{f}\left(p_{c 0}-p_{c 1}\right)
$$

where the subscripts $c 0$ and $c 1$ represent the relative values within the two cells $c 0$ and $c 1$ on either sides of the face $f$, the weighting factor $d_{f}$ is the average of the coefficients $a_{P}$ for the two neighboring cells in the linearized momentum Eq. (2.53), and $\hat{J}_{f}$ represents the mass flux influenced by the normal velocities $u_{n}$ of the two cells,

$$
\hat{J}_{f}=\rho_{f} \frac{a_{P, c 0} u_{n, c 0}+a_{P, c 1} u_{n, c 1}}{a_{P, c 0}+a_{P, c 1}}
$$

Assume that an uncorrected velocity field $\mathbf{u}^{*}$ is resolved by the momentum equation with a guessed pressure field $p^{*}$, the uncorrected mass flux $J_{f}^{*}$ is calculated according to Eq. (2.56), 


$$
J_{f}^{*}=\hat{J}_{f}^{*}+d_{f}\left(p_{c 0}^{*}-p_{c 1}^{*}\right) .
$$

The resulting mass flux does not necessary satisfy the continuity equation. Hence, it is corrected by adding a correction $J_{f}^{\prime}$,

$$
J_{f}=J_{f}^{*}+J_{f}^{\prime}
$$

Here $J_{f}$ denotes the corrected mass flux and the correction $J_{f}^{\prime}$ is calculated by

$$
J_{f}^{\prime}=d_{f}\left(p_{c 0}^{\prime}-p_{c 1}^{\prime}\right)
$$

where $p^{\prime}$ denotes the pressure correction resolved in Eq. (2.61).

The corrected mass flux should satisfy the discrete continuity Eq. (2.55). Hence, substituting Eq. (2.59) and Eq. (2.60) into Eq. (2.55), we obtain a linearized discrete equation for the pressure correction $p^{\prime}:{ }^{38}$

$$
a_{P} p^{\prime}=\sum_{n b} a_{n b} p_{n b}^{\prime}+b
$$

where $b$ represents the net flow rate into this cell,

$$
b=\sum_{f}^{N_{\text {faces }}} J_{f}^{*} A_{f}
$$


The pressure correction equation will be solved iteratively and the resolved pressure correction $p^{\prime}$ is used to correct the velocity and pressure fields.

In summary, the SIMPLE pressure velocity coupling procedure has the following steps:

- Compute the uncorrected face mass fluxes $J_{f}^{*}$ using the uncorrected velocity and pressure according to Eq. (2.56),

$$
J_{f}^{*}=\hat{J}_{f}^{*}+d_{f}\left(p_{c 0}^{*}-p_{c 1}^{*}\right) .
$$

- Solve the pressure correction equation (Eq. (2.61)) iteratively.

- Update the pressure field with a under-relaxation factor $\alpha_{p}$,

$$
p=p^{*}+\alpha_{p} p^{\prime}
$$

- Correct the face mass fluxes $J_{f}$ according to Eq. (2.59).

- Correct the velocity field according to Eq. (2.53) such as the x-component of the velocity,

$$
a_{P} u_{1}=\sum_{n b} a_{n b} u_{1, n b}^{*}+\sum_{f} p_{f}^{\prime} \mathbf{A}_{f} \cdot \hat{i}+S .
$$

- Update the flow density with the corrected pressure using the state equation. 


\subsubsection{PISO}

In the SIMPLE scheme, the pressure correction is solved using an iterative method, which is computationally expensive for transient flows. For every time step, the time step size and under-relaxation factors have to be adjusted for the convergence of the iterative procedure. To improve the computation efficiency, the PISO algorithm performs the neighbor correction and the skewness correction. The skewness correction is recommended for problems with highly distorted meshes. Thus it is not considered for the structured hexahedral meshes in this thesis. The neighbor correction usually only needs to do twice, then the corrected velocity field will satisfy not only the momentum equation but also the continuity equation more closely. Hence, though one iteration step of the PISO algorithm doing the neighbor correction takes a little more time than the SIMPLE algorithm, the number of iterations required for convergence are reduced dramatically. The following gives a detailed introduction of the neighbor correction.

Apply the Euler implicit scheme to the time derivative term, the continuity and momentum equations can be discretized as following, ${ }^{38}$

$$
\begin{aligned}
\Delta_{i} u_{i}^{n+1} & =0, \\
\frac{\rho}{\delta t}\left(u_{i}^{n+1}-u_{i}^{n}\right) & =H\left(u_{i}^{n+1}\right)-\Delta_{i} p^{n+1}+S_{i}
\end{aligned}
$$

where $\Delta$ is the difference operator, $H\left(u_{i}\right)=\sum_{n b} a_{n b} u_{i, n b}$ accounts for the influence of the 
neighboring cells and $S_{i}$ for the source. The function $H\left(u_{i}\right)$ is linear and it can be divided into a diagonal part $A_{0} u_{i}$ and another part $H^{\prime}\left(u_{i}\right)$,

$$
H\left(u_{i}\right)=A_{0} u_{i}+H^{\prime}\left(u_{i}\right)
$$

Taking the divergence of Eq. (2.64) and substituting into Eq. (2.63), the pressure equation is derived as,

$$
\Delta^{2} p^{n+1}=\Delta_{i} H\left(u_{i}^{n+1}\right)+\Delta_{i} S_{i}+\frac{\rho}{\delta t} \Delta_{i} u_{i}^{n}
$$

Denote the three intermediate values of variables between time step $n$ and $n+1$ by the superscripts $(\cdot)^{*},(\cdot)^{* *}$ and $(\cdot)^{* * *}$, the PISO predictor and corrector steps are listed as follows:

Predictor step: Calculate the uncorrected velocity field $\mathbf{u}^{*}$ using Eq. (2.64) and Eq. (2.65) based on the pressure and velocity values at time step "n",

$$
\left(\frac{\rho}{\delta t}-A_{0}\right) u_{i}^{*}=H^{\prime}\left(u_{i}^{*}\right)-\Delta_{i} p^{n}+S_{i}+\frac{\rho}{\delta t} u_{i}^{n}
$$

First corrector step: Substitute Eq. (2.67) into the Eq. (2.66), a new pressure field $p^{*}$ is solved by the resulting equation,

$$
\Delta_{i}\left[\left(\frac{\rho}{\delta t}-A_{0}\right)^{-1} \Delta_{i}\right]\left(p^{*}-p^{n}\right)=\Delta_{i} u_{i}^{*}
$$


Then, subtract the momentum equation (Eq. (2.64)) by Eq. (2.67) and obtain a new velocity field $\mathbf{u}^{* *}$,

$$
\left(\frac{\rho}{\delta t}-A_{0}\right)\left(u_{i}^{* *}-u_{i}^{*}\right)=-\Delta_{i}\left(p^{*}-p^{n}\right)
$$

Second corrector step: Similarly, a new pressure field $p^{* *}$ and a new velocity field $\mathbf{u}^{* * *}$ are calculated using Eqs. (2.70) and (2.71) below

$$
\begin{aligned}
\Delta_{i}\left[\left(\frac{\rho}{\delta t}-A_{0}\right)^{-1} \Delta_{i}\right]\left(p^{* *}-p^{*}\right) & =\Delta_{i}\left[\left(\frac{\rho}{\delta t}-A_{0}\right)^{-1} H^{\prime}\left(u_{i}^{* *}-u_{i}^{*}\right)\right] \\
\left(\frac{\rho}{\delta t}-A_{0}\right)\left(u_{i}^{* * *}-u_{i}^{* *}\right) & =H^{\prime}\left(u_{i}^{* *}-u_{i}^{*}\right)-\Delta_{i}\left(p^{* *}-p^{*}\right)
\end{aligned}
$$

Usually two corrector steps are sufficient for the accuracy requirement, although the number of corrector steps can be adjusted for further refinement. In this work, 2 corrector steps will be used as default. 


\section{Simulation of flows over a backward- facing step}

\subsection{Introduction}

In this chapter, we are interested in the comparison of RANS and LES in the laminar flow limit in a benchmark problem, namely the flow over a backward facing step. Four typical eddy viscosity turbulence models, the $k-\varepsilon$, the RNG $k-\varepsilon$, the LES Smagorinsky and the LES one equation models, are investigated.

The laminar flow over a backward-facing step becomes turbulent with increasing value of the Reynolds number. The flow is identified as laminar when $R e<1200$, as transitional for $1200<\operatorname{Re}<6600$, and as turbulent when $R e>6600 .{ }^{1}$ Here, the Reynolds number is calculated by

$$
\operatorname{Re}=\frac{V D}{v}
$$

where the characteristic length $D$ is twice the step length, i.e., $D=2 S$. The characteristic velocity $V=\frac{2}{3} U$, where $U$ denotes the maximum value of the prescribed parabolic inflow velocity profile. ${ }^{1}$ 
The isothermal air flows over a backward-facing step with $R e=389$ and 1000 are simulated, and the numerical results are compared with the experimental results from Ref. ${ }^{20}$ The flows are laminar or nearly transient, and their motions are resolved by the Navier-Stokes equations Eq. (2.1) and Eq. (2.4).

As discussed in Chapter 2, the popular turbulence models for RANS simulations include the standard $k-\varepsilon$ model and the RNG $k-\varepsilon$ model, and the Smagorinsky model and the LES one-equation model are popular SGS models for LES. In order to investigate the behavior of these turbulence models for low Reynolds number flows, that is, in the transient and laminar flow regime, computations have been performed with the turbulence models turned on and off. Turning the turbulence models off has been achieved by setting the turbulent viscosity to zero. ${ }^{44}$

In OpenFOAM, turbFoam and oodles are used as the RANS and LES solvers, respectively. turbFoam uses the $k-\varepsilon$ and RNG $k-\varepsilon$ turbulence models with the standard wall-functions. oodles is the LES solver using the Smagorinsky and the one-equation LES turbulence models. Both turbFoam and oodles use the PISO (pressure-implicit split-operator) ${ }^{40}$ algorithm as their iterative solver. In the laminar flow simulations, the turbulence models in turbFoam and oodles are switched off by forcing the turbulent viscosity to be zero. More details about OpenFOAM can be found in Ref. ${ }^{30}$ and Appendix A.

It is observed that the LES predictions yield recirculation zones which are in acceptable agreement with the experimental results, while the RANS simulations are not. Also, the 
LES computations are consistent with the corresponding laminar flows, whereas the turbulent RANS simulations produce inconsistent results.

\subsection{Flow configuration and initial conditions}

The geometry parameters of the $x-y$ cross section of the backward-facing step are shown in Fig. 3.1. The walls on the top and the bottom are assumed to be none slip. The inflow velocity profiles were obtained from Williams et al., ${ }^{1}$ and the gradient of the inflow velocity is assumed to be zero. The pressure at the outflow boundary is assumed to be zero. The depth

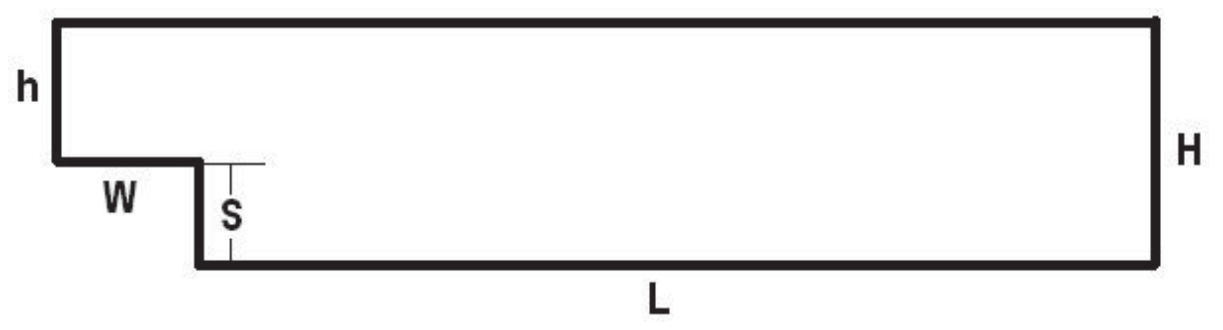

Figure 3.1: Backward-facing step geometry, ${ }^{1} S=4.9 \mathrm{~mm}, h=5.2 \mathrm{~mm}, H=$ $10.1 \mathrm{~mm}, W=5 \mathrm{~mm}, L=300 \mathrm{~mm}$.

of the step in $z$ direction is assumed to be deep enough such that all simulations have been performed for a two-dimensional mesh. In fact, a comparison between a three-dimensional and a two-dimensional computation has shown that the two simulations were almost identical. Additionally, in the LES simulations, the nonuniform mesh with denser grid points near the wall is used instead of a wall function, whereas for the RANS simulations, wall functions have been used. 


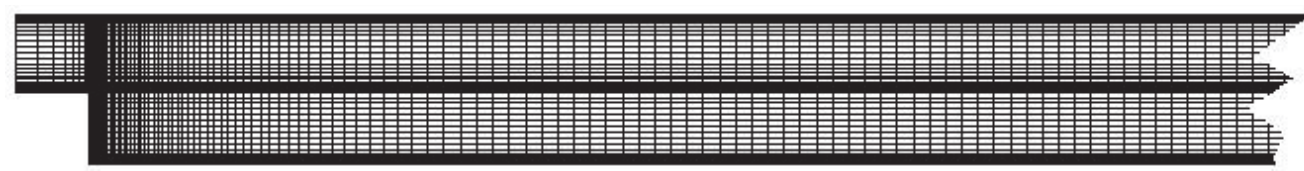

Figure 3.2: $2 \mathrm{D}$ Nonuniform Mesh, $8 \times 22$ before step and $312 \times 40$ after step.

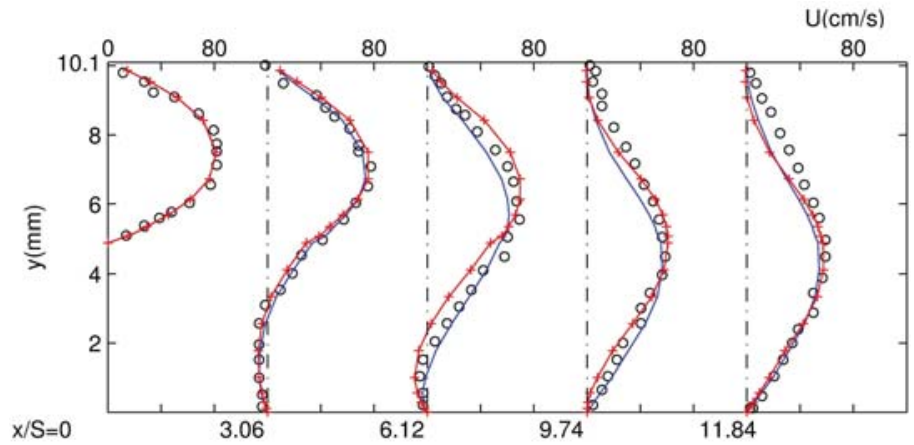

(a) Experiment, o; LES Laminar, -; RANS Laminar, +-

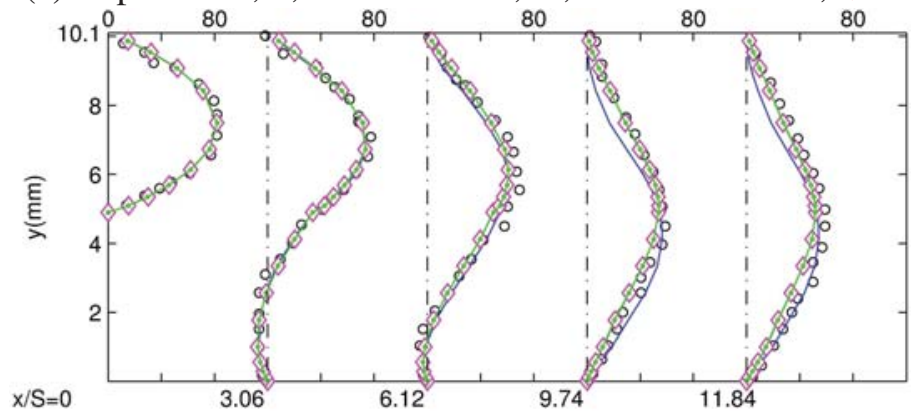

(b) LES: Laminar, - ; Smagorinsky, $\diamond-$; OneEq, .-

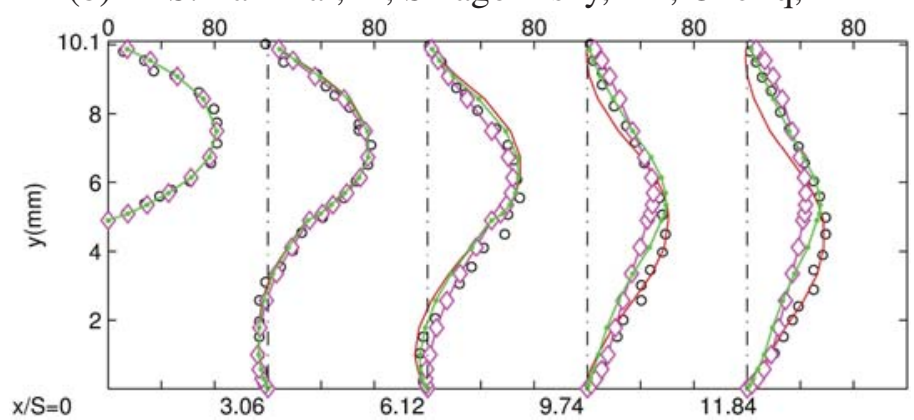

(c) RANS: Laminar, $-; k-\varepsilon,+-$; RNG $k-\varepsilon, \diamond-$

Figure 3.3: Experimental and computed velocity profiles for $\operatorname{Re}=389$ at different $x / S$-locations 


\subsection{LES/RANS results and discussion}

The computation results with the LES/RANS turbulence models turned on and off are compared with the experimental data from Armaly et al. ${ }^{20}$ The comparison and discussion will be done for the $\mathrm{Re}=389$ case and the $\mathrm{Re}=1000$ case separately. Note that the LES results in Figs. 3.3-3.8 are time averaged values such that they can be compared to the RANS results.

\section{$\mathrm{Re}=389$ case}

As mentioned above, the flow with Reynolds number $R e=389$ is laminar. Hence, the simulation results with the turbulence models switched off are investigated and compared with the experimental results first. Figure 3.3(a) shows that the simulation results for the RANS and LES, with the turbulence models switched off, are comparable.

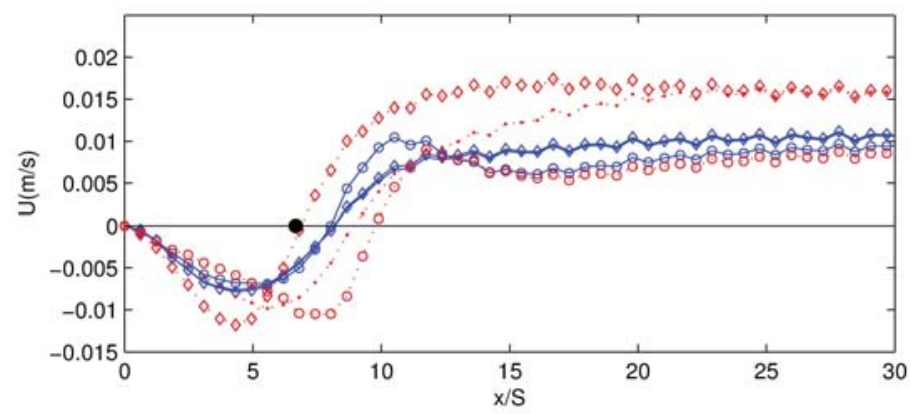

Figure 3.4: Velocity near the lower wall for $R e=389$. Experimental reattachment point, •; LES: Laminar, ॰-; Smagorinsky, $\diamond-$; OneEq, •-; RANS: Laminar, $\circ--; k-\varepsilon, \diamond--;$ RNG $k-\varepsilon, \cdot--$ 
Figure 3.3(b) compares the LES results with the turbulence models switched off and on. It is seen that the agreement between the three different cases is quite good and that the experimental data are well matched. Figure 3.3(c) compares the RANS results with the turbulence models switched off and on. Here, the agreement between the three different cases is still acceptable, but not as good as for the LES simulations.

Figure 3.4 shows the reattachment point after the step, that is, at the end of the recirculation zone. This point is obtained by looking at the change in sign of the horizontal velocities near the lower wall. As can be seen, there are considerable differences between the LES and the RANS simulations. The standard RANS $k-\varepsilon$ model shows the best agreement with the experimental value, but the other RANS simulations are far off. The LES simulations, with or without the turbulence models, have almost identical reattachment points, which shows that the LES computations are consistent for the laminar, low Reynolds number flow. The good agreement of the $k-\varepsilon$ model with the experimental attachment point could be purely coincidental. To clarify this issue, simulations at the higher Reynolds number, $\operatorname{Re}=1000$, are performed.

\section{$\mathrm{Re}=\mathbf{1 0 0 0}$ case}

The flow with Reynolds number $R e=1000$ is near the transient flow regime which starts at $R e=1200$. From Fig. 3.5(a) it is seen that the laminar description is not well suited in this almost transient flow regime. 
Figure 3.5(b) confirms again the consistency of the LES turbulence models with the laminar flow. Furthermore, from Fig. 3.5(c), it is seen that the RANS turbulence models perform poorly when compared with the RANS laminar simulation, or with the experiments. In fact, the turbulent RANS simulations show a fully developed turbulence velocity profile half-way down the channel. This is a clear indication, that the RANS turbulence models are not consistent for low Reynolds number flows.

Figure 3.6 shows the locations of the reattachment points. Again, as in the $\mathrm{Re}=389$ case, the LES simulations exhibit a consistent behavior, but they underpredict the reattachment point. The RANS simulations are again very inconsistent; this time, the laminar RANS gave the best agreement. This figure confirms the observation made by Williams et al., ${ }^{1}$ namely, that the two dimensional simulation typically underestimates the primary reattachment separation region for $R e>400$.

In addition to the recirculation zone at the bottom wall after the step, there is a second recirculation zone at the top wall. This is illustrated with the LES one-equation turbulence computation in Fig. 3.7(a). In contrast, the RANS RNG $k-\varepsilon$ model, shown in Fig. 3.7(b), is unable to predict this second recirculation zone. This is confirmed in Fig. 3.8, which shows that both RANS turbulence models are not able to predict the upper recirculation zone, whereas the RANS laminar simulation does. The LES simulations on the other hand, show again consistent behavior with the turbulence models off and on. The detachment point in this case is underpredicted again. 


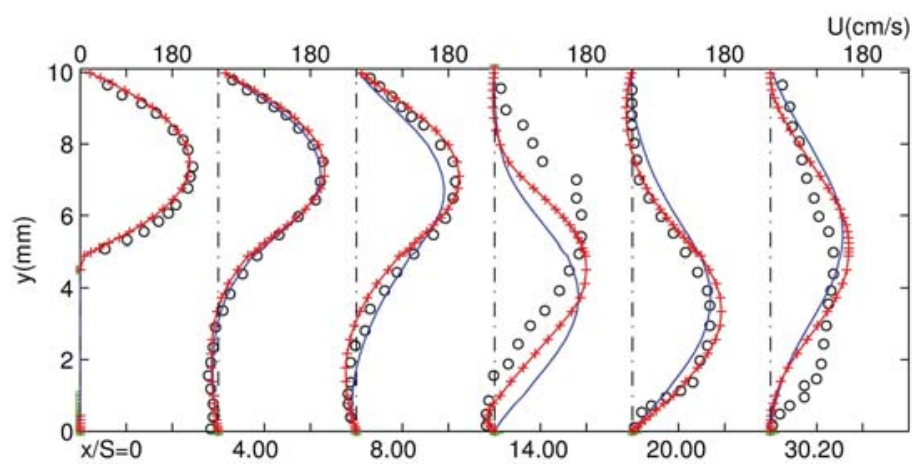

(a) Experiment, o; LES Laminar, -; RANS Laminar, +-

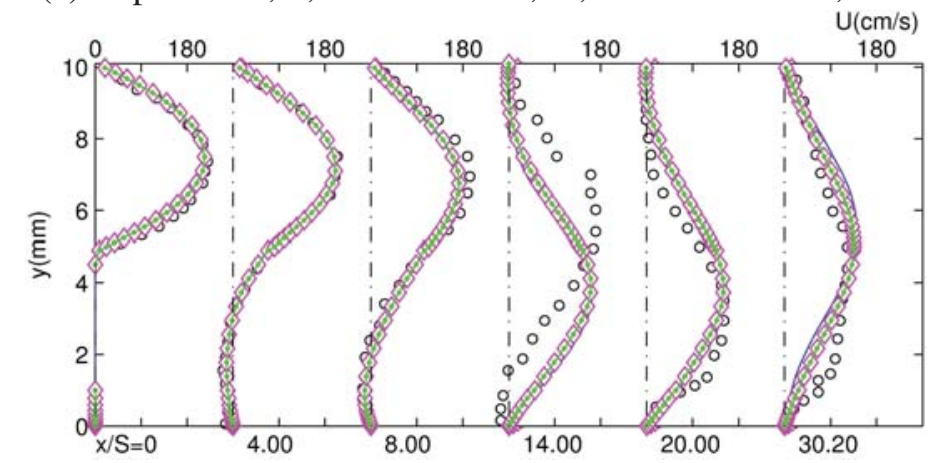

(b) LES: Laminar, -; Smagorinsky, $\diamond-;$ OneEq, .-

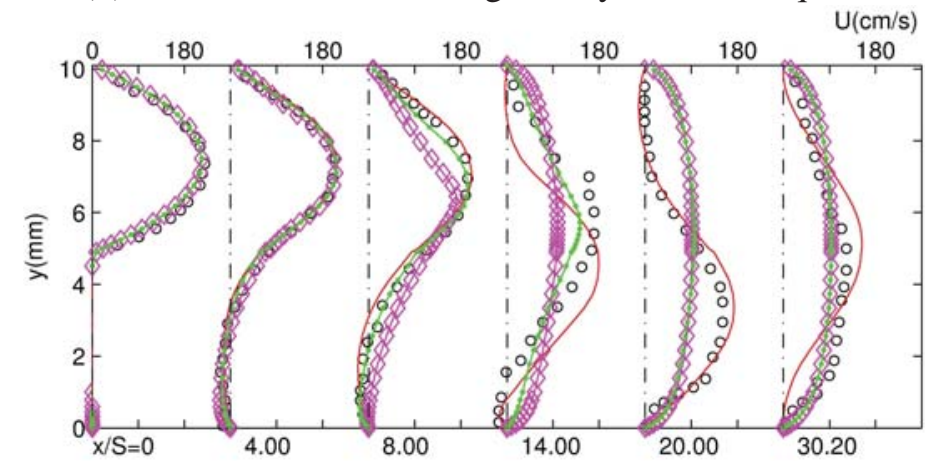

(c) RANS: Laminar, $-; k-\varepsilon,+-$; RNG $k-\varepsilon, \diamond-$

Figure 3.5: Experimental and computed velocity profiles for $\mathrm{Re}=1000$ at different $x / S$-locations

\subsection{Conclusion}

Comparisons of LES and RANS simulations of the two-dimensional flows at $\operatorname{Re}=389$ and $\mathrm{Re}=1000$ have been performed. The velocity profiles at various locations along the channel and the reattachment points of recirculation zones have been investigated and compared. 


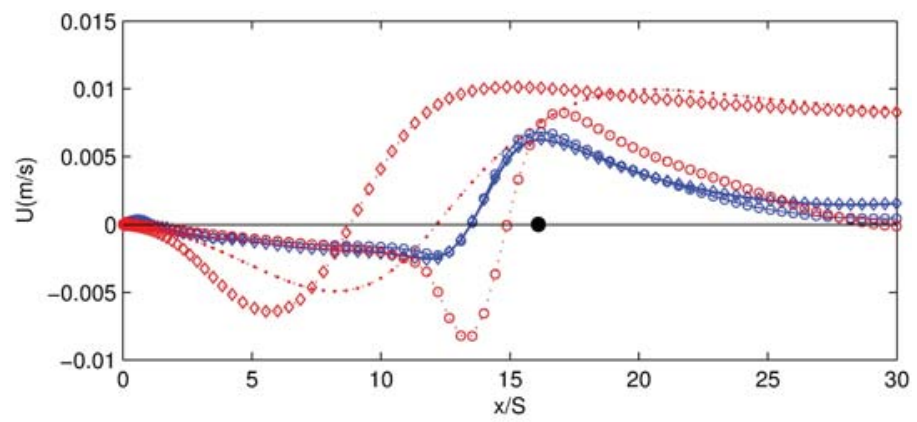

Figure 3.6: Velocity near the lower wall for $\mathrm{Re}=1000$. Experimental reattachment point, •; LES: Laminar, ॰-; Smagorinsky, $\diamond-$; OneEq, •-; RANS: Laminar, $\circ--; k-\varepsilon, \diamond--;$ RNG $k-\varepsilon, \cdot--$

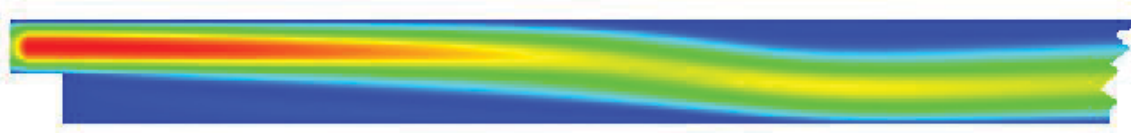

(a) LES, OneEq

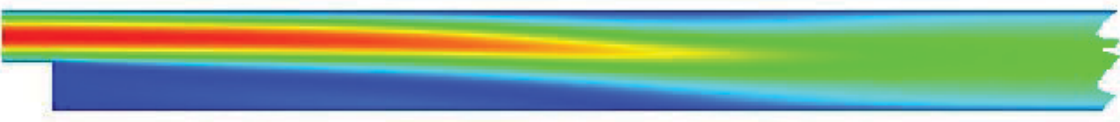

(b) RANS, RNG k-epsilon

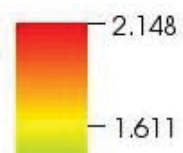

$-1.074$

$-0.5371$

0.0000

Figure 3.7: Velocity color plot of $u_{x}$ for $\mathrm{Re}=1000$

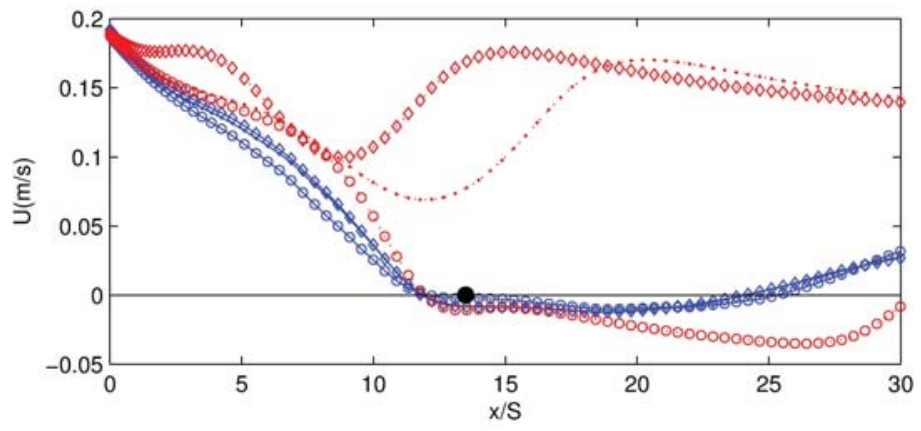

Figure 3.8: Velocity near the top wall for $\mathrm{Re}=1000$. Experimental detachment point, •; LES: Laminar, ॰-; Smagorinsky, $\diamond-$; OneEq, •-; RANS:

Laminar, $\circ--; k-\varepsilon, \diamond--; \mathrm{RNG} k-\varepsilon, \cdot--$

For the lower Reynolds number, both, the RANS and the LES results were in good agreement with the experiments, but the LES computations were more consistent, especially for 
the reattachment point. For the higher Reynolds number case, the RANS simulations performed poorly, whereas the LES computations were again consistent and showed generally good agreement with the experiments. In particular, the second recirculation zone at the top of the channel wall was quite well predicted by the LES simulations, whereas the RANS turbulence models were not able to even detect the second recirculation zone.

Overall, the LES turbulence models under consideration showed consistency in the laminar and near transient flow regimes, whereas the RANS turbulence models performed poorly. 


\section{Simulation of fuel sprays}

CFD-based computer simulations have become a standard tool for investigating flow, spray and combustion phenomena in engines. One of the key issues is the correct description of turbulence. While Reynolds-averaged Navier-Stokes (RANS) methods are widely used in such computations, large eddy simulations (LES) have become increasingly more impor$\operatorname{tant}^{8,10,45}$

This chapter is focused on the comparison of RANS and LES turbulence modeling of high injection pressure diesel sprays under non-evaporating and evaporating conditions. First, the simulation results have been validated with experimental data from diesel fuel sprays at different ambient gas pressures. Then comparisons have been performed between LES and traditional RANS simulations. The data that have been used in the comparisons include the spray tip penetration, the drop size, the vorticity, the turbulent kinetic energy and viscosity.

A spray is composed of a dispersed liquid phase and a continuous gaseous phase. As a typical multi-phase flow, the simulation of spray requires to solve the governing equations for the gaseous and the liquid phase.

The Euler-Lagrangian approach is used here for the numerical calculation of the spray. The conservation equations governing the gas flow are solved under the Eulerian framework: 
the spatial domain is divided into many small cells composing the mesh, and the fluid density, velocity, pressure and enthalpy are computed and stored on on each computation cell. Correspondingly, the liquid phase is modeled by the Lagrangian approach: the position of the liquid drops are described and tracked by the Lagrangian coordinates.

\subsection{Liquid phase modeling}

The fuel atomization and spray process is a complex phenomenon. The fuel drops exchange mass, momentum and heat with not only the neighboring drops but also with the surrounding gas flow. The gas phase can influence the liquid phase via drag and turbulence. Inversely, the droplet effect on the gas can also be modeled by introducing the spray source terms to the fluid phase governing equations, i.e. Eqs. (4.24)-(4.26). This is called the two-way coupling of liquid and gas phase, which considers both the droplet and gas effect on each other. In dilute sprays (liquid volume fraction $\phi_{v, l}<10^{-3}$ ), ${ }^{37}$ the liquid dynamics are mainly influenced by the gas turbulence and the droplet effect on the gas can be ne-

glected. This coupling is referred to as one-way coupling. For dense sprays $\left(\phi_{v, l}>10^{-3}\right)$, the interaction between droplets leads to the drop collision and coalescence, i.e., four-way coupling. ${ }^{46}$

In the sprays discussed here, the liquid state fuel drops are forced into a chamber through a pressure-driven nozzle. As a result, the fuel drops are atomized and then broken into a fine misted spray. The liquid volume fraction is dense near the nozzle and thus drop collision 
and coalescence (four-way coupling) are considered. For the computational cases with the same geometry and similar injection parameters with the near-nozzle case, the liquid volume fraction is dilute or nearly zero in most of the computational cells. Thus, the spray source terms in the turbulence model equations are neglected, i.e., turbulence modeling is one-way coupling. A discussion of the two-way coupling via spray source terms in the RNG $k-\varepsilon$ equations is presented in Appendix B and Refs. ${ }^{47,48}$

The concept of a parcel is introduced for the description of the liquid phase. The number of drops is extremely large, and it is impossible to track all drops with present computer capacities. A parcel is a group of drops with exactly the same properties. The mass of the parcel is the sum of the mass of all component drops, and any drop in one parcel follows the same ordinary differential equations. ${ }^{37,49}$ These equations describe the trajectory, mass, momentum, and heat transfer of single parcels. The liquid drops are represented

by a finite number of parcels, and a stochastic model is proposed for modeling the density distributions of a parcel by a probability function, $f(t, X)$, where $t$ denotes the time and $X=\left(\mathbf{x} ; \mathbf{v} ; r ; T_{d} ; y ; \dot{y}\right)$ is the droplet state consisting of drop position, velocity, radius, temperature, deformation and deformation rate. The evolution of the spray is given by the spray evolution equation, ${ }^{50}$

$$
\frac{\partial f}{\partial t}+\nabla_{X} \cdot(f X)=\dot{f}_{c o l l}+\dot{f}_{b u}
$$

where $\dot{f}_{\text {coll }}$ and $\dot{f}_{b u}$ are the droplet collision and breakup source terms. 
In the computation tool, OpenFOAM, the liquid phase is modeled by several distinctive submodels: ${ }^{30}$ droplet injection, breakup, drag, dispersion, collision and evaporation models. Each of these submodels is discribed below. Note that the droplet injection model and the Cascade Atomization and Breakup (CAB) model are not standard models in OpenFOAM, but have been implemented into the code as part of this thesis. The implementation of these models is documented in Appendix A.1. Further, the implementation of LES in the context of sprays, as used in the simulations, is documented in Appendix A.2.

\subsubsection{Droplet injection}

The injection system delivers fuel to the combustion process, which, in this thesis, takes place in a constant-volume cylindrical-shaped vessel. In the program implementation, the fuel parcels are injected according to an initial drop size distribution (IDSD), which is introduced to account for the droplet surface stripping near the nozzle. ${ }^{51}$ The corresponding IDSD of the parcels is given by,

$$
g(r)= \begin{cases}\frac{n+4}{r_{0}}\left(\frac{r}{r_{0}}\right)^{n+3} & \text { if } 0<r \leq r_{0} \\ 0 & \text { otherwise }\end{cases}
$$

where $n \geq 0$ is a parameter, $r$ and $r_{0}$ are the drop and nozzle radii, respectively. The parameter $n$ is tuned to be 0.5 for this work. 


\subsubsection{Droplet breakup}

The liquid fuel drops interact with the gas near the nozzle, and this aerodynamic interaction leads to the primary drop breakup (or atomization), i.e., the first drop breakup after injection. This interaction assumed to happen after a delay of the breakup time, $t_{b u}$, which is defined as ${ }^{52,53}$

$$
t_{b u}=C_{\lambda} \sqrt{\frac{\rho_{l}}{\rho_{g}}} \frac{D}{u_{0}},
$$

where $u_{0}$ is the jet exit velocity, $D$ is the nozzle diameter, $\rho_{l}$ is the liquid phase density, $\rho_{g}$ is the gas phase density and $C_{\lambda}$ is a nozzle dependent constant taken to be $C_{\lambda}=5.5$.

If the injection time of one drop is less than the above breakup time, the drop is assumed to be in the range of liquid core, whose existence motivates the development of the $\mathrm{CAB}$ drop breakup model, ${ }^{51}$ which is realized into OpenFOAM as an user-defined library (details refer to Appendix A.1.3). The CAB model is based on the breakup condition of the standard TAB model. ${ }^{54}$ The drop distortion is forced by the aerodynamic gas-liquid interactions, damped by the liquid fuel viscosity $\mu_{l}$ and restored by the drop surface tension $\sigma$. To describe the drop distortion quantitatively, a deformation parameter, $y$, is defined as $y=2 x / a$, where $x$ is the radial cross-sectional change from its equilibrium position and $a$ is the drop radius. The parameter, $y$, satisfies the deformation equation, which is given as

$$
\ddot{y}+\frac{5 \mu_{l}}{\rho_{l} a^{2}} \dot{y}+\frac{8 \sigma}{\rho_{l} a^{3}} y=\frac{2 \rho_{g}\left\|\mathbf{u}_{r e l}\right\|^{2}}{3 \rho_{l} a^{2}},
$$


where $\mathbf{u}_{r e l}$ denotes the relative drop-gas velocity, and the subscripts $(\cdot)_{l}$ and $(\cdot)_{g}$ represent the liquid or gas phase variables, respectively.

The $\mathrm{CAB}$ model considers the aerodynamic forces acting on a droplet which lead to its distortion and subsequent breakup into a collection of product droplets. The droplet will break up under the condition that the deformation parameter $y>1$. The product droplets follow a uniform size distribution,

$$
a=a_{0} e^{-K_{b u} t_{b u}}
$$

where $a_{0}$ is the radii of the product droplet and $K_{b u}$ is the breakup frequency.

The expression of the droplet breakup frequency $K_{b u}$ is given by:

$$
K_{b u}= \begin{cases}k_{1} \omega, & \text { if } W e_{c r i t}<W e \leq W e_{b, s} \\ k_{2} \omega \sqrt{W e}, & \text { if } W e_{b, s}<W e \leq W e_{s, c} \\ k_{3} \omega W e^{3 / 4}, & \text { if } W e>W e_{s, c}\end{cases}
$$

where the droplet oscillation frequency $\omega$ is given by

$$
\omega^{2}=\frac{\sigma}{\rho_{l} d^{3}}-\frac{100 \mu_{l}^{2}}{\rho_{l}^{2} d^{4}}
$$


where $\mu_{l}$ represents the liquid viscosity. The Weber number,

$$
W e=\frac{\rho_{g} a\left\|\mathbf{u}_{r e l}\right\|^{2}}{\sigma}
$$

depends on the gas density $\rho_{g}$, the magnitude of the drop-gas relative velocity $\mathbf{u}_{r e l}$ and the liquid surface tension $\sigma$. Here, $W e_{c r i t}=6, W e_{b, s}=80$, and $W e_{s, c}=350 .{ }^{51,55}$ Weber number is the controlling parameter in the three breakup regimes: the bag breakup regime $\left(W e_{c r i t}<W e \leq W e_{b, s}\right)$; the stripping breakup regime $\left(W e_{b, s}<W e \leq W e_{s, c}\right)$; and the catastrophic breakup regime $\left(W e>W e_{s, c}\right) .{ }^{51}$

\subsubsection{Droplet drag}

The gas flow affects the liquid state drop position and velocity via the drag force, which is modeled by: ${ }^{56}$

$$
\begin{aligned}
\frac{d \mathbf{x}_{d}}{d t} & =\mathbf{u}_{d} \\
\frac{d \mathbf{u}_{d}}{d t} & =\frac{\mathbf{u}-\mathbf{u}_{d}}{\tau}
\end{aligned}
$$

where $\mathbf{x}_{d}$ represents the droplet position, $\mathbf{u}_{d}$ the droplet velocity, $\mathbf{u}$ the flow velocity, and $\tau$ the momentum relaxation timescale.

The momentum relaxation timescale measures the magnitude of the interaction between the droplets and the surrounding gas. ${ }^{57}$ It depends on the magnitude of the relative velocity 
$U_{r e l}=\left\|\mathbf{u}_{r e l}\right\|=\left\|\mathbf{u}-\mathbf{u}_{d}\right\|,{ }^{47,58}$ and is given by

$$
\tau=\frac{4 \rho_{l} d}{3 \rho_{g} C_{D} U_{r e l}}
$$

where $d$ denotes the droplet diameter, $\rho_{l}$ the liquid fuel density, $\rho_{g}$ the gas flow density, and the drag coefficient $C_{D}$ is given by ${ }^{47,58}$

$$
C_{D}= \begin{cases}\frac{24}{R e_{d}}\left(1+\frac{1}{6} R e_{d}^{2 / 3}\right), & \text { if } R e_{d}<1000 \\ 0.424, & \text { otherwise }\end{cases}
$$

Here the drop Reynolds number $R e_{d}$ is given by

$$
R e_{d}=\frac{d \rho_{g} U_{r e l}}{\mu_{g}}
$$

Then the formulation of the momentum relaxation time $\tau$ can be written as

$$
\tau=\frac{4 \rho_{l} d^{2}}{3 R e_{d} \mu_{g} C_{D}}
$$

A high $\tau$ indicates a smaller drop acceleration. Generally, a droplet with high $\tau$ is large, and essentially undisturbed by the surrounding gas. Inversely, low values of $\tau$ correspond to small droplets which follow the gas motion completely. 


\subsubsection{Droplet evaporation}

In evaporating sprays, heat is transferred from the hot gas to the cold droplet. Once the droplet temperature reaches the critical temperature, the liquid state droplet evaporates and turns into gaseous form, which leads to the mass transfer from the liquid phase to the gas phase. The evaporation rate is indicated by the vapor pressure, which is the pressure of a vapor in thermodynamic equilibrium with its liquid phase.

The evaporation process is modeled by the following mass and energy conservation equations for single parcels: ${ }^{57}$

$$
\begin{aligned}
\frac{d m_{p}}{d t} & =-\frac{m_{p}}{\tau}, \\
\frac{d T_{p}}{d t} & =\frac{f}{\tau_{h}}\left(T-T_{p}\right)-\frac{1}{c_{l} \tau h_{v}\left(T_{p}\right)} .
\end{aligned}
$$

Here $m_{p}$ is the mass of the parcel, $T_{p}$ the temperature of the parcel, $T$ the surrounding gas temperature, $c_{l}$ the liquid fuel specific heat capacity, and $h_{v}$ is the latent heat of vaporization. The factor $f$ corrects the amount of heat exchange due to the mass transfer, ${ }^{59}$ and is calculated by

$$
f=\frac{z}{e^{z}-1} \text { with } z=-\frac{c_{p, v} \frac{d m_{p}}{d t}}{\pi D_{d} k_{h} N u},
$$

where $c_{p, v}$ is the fuel vapor specific heat at constant pressure, $k_{h}$ is the mixture thermal conductivity, and $N u$ is the nondimensional Nusselt number defined in Eq. (4.21). 
The relaxation times, $\tau$ and $\tau_{h}$, denote the evaporation relaxation time and the heat transfer relaxation time, respectively,

$$
\begin{aligned}
\tau & =\frac{\rho_{l} d^{2}}{6 \rho_{v} \operatorname{Sh} \mathscr{D} \ln \frac{P-P_{v, \infty}}{P-P_{v, s}}} \\
\tau_{h} & =\frac{\rho_{l} c_{l} d^{2}}{6 k_{h} N u}
\end{aligned}
$$

where $\rho_{l}$ is the liquid droplet density, $\rho_{v}$ is the fuel vapor density, $\mathscr{D}$ is the mass diffusivity, $S h$ is the nondimensional Sherwood number defined in Eq. (4.20), $P$ is the total pressure of the mixture of the air and fuel vapor, and $P_{v, s}$ and $P_{v, \infty}$ are the partial pressure of the fuel vapor at the droplet surface and far from it, respectively.

The Sherwood number represents the ratio of convective to diffusive mass transport, which is correlated as, ${ }^{60}$

$$
S h=2.0+0.6 R e^{1 / 2} S c^{1 / 3}
$$

where $S c=v_{g} / \mathscr{D}$ is the gas phase Schmidt number at the mean temperature, $T_{m}=\left(T_{l}+\right.$ $T) / 2$, i.e. the average of the liquid droplet and the gas temperature.

The Nusselt number, $\mathrm{Nu}$, analogs the Sherwood number for heat transfer, which is calculated by the Ranz-Marshall correlation, ${ }^{60}$

$$
N u=2.0+0.6 \operatorname{Re}^{1 / 2} \operatorname{Pr}^{1 / 3}
$$


where $\operatorname{Pr}=c_{p} \mu_{m} / k_{h}$ is the Prandtl number at the temperature $T_{m}$. Here $c_{p}$ denotes the fuel specific heat, and $\mu_{m}$ is the fuel viscosity at temperature $T_{m}$.

\subsubsection{Droplet dispersion}

The real gas velocity $\mathbf{u}^{o}$ is the sum of the time-averaged or spatially filtered gas velocity $\mathbf{u}$ and its fluctuation $\mathbf{u}^{\prime}$, i.e., $\mathbf{u}^{o}=\mathbf{u}+\mathbf{u}^{\prime}$. Then the relative velocity, $\mathbf{u}_{r e l}=\mathbf{u}-m a t h b f u_{d}$ in Eq. (4.10), can be written as,

$$
\mathbf{u}_{r e l}=\left(\mathbf{u}_{d}-\mathbf{u}\right)-\mathbf{u}^{\prime}
$$

The velocity fluctuation, $\mathbf{u}^{\prime}$, oscillates the droplet drag force, and the resulting instabilities lead to disturbances in the gas flow.

In the RANS simulations, the turbulent kinetic energy $k$ is related to the magnitude of the velocity fluctuation $\mathbf{u}^{\prime}$ in Eq. (2.23). Hence, the magnitude of the velocity fluctuation is determined by sampling of a Gaussian distribution with a variance equal to $\sqrt{2 k / 3}$ and zero mean. The direction of the velocity fluctuation $\mathbf{u}^{\prime}$ is also chosen randomly. From the last sampling, once the time passed is greater than the characteristic time $\tau_{\text {turb }}$, a new sampling is done and the value of the velocity fluctuation is updated. The characteristic time $\tau_{\text {turb }}$ is defined as: ${ }^{47,61}$

$$
\tau_{\text {turb }}=\min \left[\frac{k}{\varepsilon}, \frac{k^{3 / 2}}{\varepsilon} \frac{C_{\mu}^{3 / 4}}{\left\|\mathbf{u}_{r e l}\right\|}\right]
$$


with the constant $C_{\mu}=0.16432 .{ }^{30}$

Large eddy simulation resolves the spatial filtered gas velocity, and the unresolved velocity fluctuation in the subgrid scale is relatively small. The effect of $\mathbf{u}^{\prime}$ on the drag force can be neglected. In this thesis, the dispersion model is turned off in the LES simulation.

\subsubsection{Droplet collision}

Droplets tend to collide where the droplet number density is high. In spray simulations, there is a large quantity of droplets near the nozzle. Hence, the droplet collision model is important in the investigation of the fuel spray. During the collision process, two drops exchange their mass, momentum and energy.

When two droplets impinge on each other, there are three possible outcomes: ${ }^{62-64}$ bounce, coalescence and separation. If the relative velocity of the droplets is smaller than the pressure force in the gap between them, then the two drops do not contact and repel away from each other, i.e., they bounce off each other. If the relative velocity is large enough, then the drops coalesce to form one drop, and this coalescence process is responsible for the increase of the drop size. Finally, if the drops have excess kinetic energy, then temporary coalescence occurs and the two drops separate from each other or break up into numerous smaller droplets. 
In this study, the Nordin collision model is used instead of the famous O' Rourke model. The O' Rourke's droplet collision model ${ }^{65}$ assumes that two parcels may collide with each other if they lie in the same computation cell. Under this assumption, two parcels in different computation cells have no chance to collide even if they are very close to each other. Nordin's collision model ${ }^{66}$ avoids this mesh dependence by assuming that the collisions may occur if the trajectories of the two parcels intersect and they reach this intersection point at the same time. Moreover, the O' Rourke model ignores the preference of the

droplet direction, which is important in sprays with large void fraction in one cell. ${ }^{66}$ In OpenFOAM, Nordin's model is used as the trajectory collision model.

\subsection{Gaseous phase modeling}

The gaseous phase in the spray process is also governed by the Navier-Stokes equations. According to Chapter 2, the time-averaged and the spatial-filtered Navier-Stokes have the same form. Here, the time-averaged or the spatial filtered governing equations for the gaseous phase are given as,

$$
\begin{aligned}
\frac{\partial \rho}{\partial t}+\frac{\partial \rho u_{j}}{\partial x_{j}} & =S_{m} \\
\frac{\partial \rho u_{i}}{\partial t}+\frac{\partial \rho u_{i} u_{j}}{\partial x_{j}} & =-\frac{\partial p}{\partial x_{i}}+\frac{\partial}{\partial x_{j}}\left(\tau_{i j}+\tau_{i j}^{t u b}\right)+S_{u} \\
\frac{\partial(\rho h)}{\partial t}+\frac{\partial\left(\rho h u_{j}\right)}{\partial x_{j}} & =\frac{\partial}{\partial x_{k}}\left[\left(\frac{\mu}{P r}+\mu_{t}\right) \frac{\partial h}{\partial x_{k}}\right]+\frac{D p}{D t}+S_{\mathrm{h}}
\end{aligned}
$$


where $h$ is the enthalpy, the density $\rho$ and the pressure $p$ are Reynolds averaged or filtered, and the other variables are all Favre averaged or filtered. The Prandtl number $\operatorname{Pr}=\mu c_{p} / K$ depends on the fluid properties, where $c_{p}$ represents the heat capacity and $K$ denotes the thermal conductivity. The turbulent Prandtl number $\operatorname{Pr}_{\mathrm{t}} \approx 1$. The material derivative of the pressure is defined as

$$
\frac{D p}{D t} \equiv \frac{\partial p}{\partial t}+u_{j} \frac{\partial p}{\partial x_{j}} .
$$

The effects of the droplets on the fluid flow field are modeled in the gas-phase equations by the spray source terms $S_{\phi}$, where $\phi$ is $m, u$ or $h$, denoting mass, momentum and energy, respectively. In each computational cell, the rate of change of mass, momentum or energy is added over all droplets in this cell, ${ }^{47}$ i.e. the spray source term $S_{\phi}$ sums up the rate change of $\rho \phi$ of all droplets with the following form in any computational cell:

$$
\begin{aligned}
S_{\phi p}[\text { cellI }] & =\sum_{d}\left(m_{d}^{o} \phi_{d}^{o}-m_{d}^{n} \phi_{d}^{n}\right) /\left(V_{\text {cellI }} \Delta t\right), \\
& =\sum_{d}\left[m_{d}^{o} \frac{\left(\phi_{d}^{o}-\phi_{d}^{n}\right)}{\Delta t}+\phi_{d}^{n} \frac{\left(m_{d}^{o}-m_{d}^{n}\right)}{\Delta t}\right] /\left(V_{\text {cellI }}\right), \\
& =\sum_{d}-\left(m_{d}^{o} \dot{\phi}_{d}+\dot{m}_{d} \phi_{d}^{n}\right) /\left(V_{\text {cellI }}\right),
\end{aligned}
$$

where the subscript $(\cdot)_{d}$ represents the value of each droplet, the superscript $(\cdot)^{o}$ and $(\cdot)^{n}$ represents the value at time $t$ and time $t+\Delta t$ respectively, $m_{d}$ denotes the droplet mass and $V_{\text {cellI }}$ is the volume of cellI. The first part of Eq. (4.27) models the interaction force between the fluid flow and the fuel droplets. The second part described the change caused by the vaporization momentum flux. ${ }^{67}$ 
The mean total stress tensor $\tau_{i j}^{t o t}$ is the sum of the mean stress tensor $\tau_{i j}$ and the mean turbulence stress tensor $\tau_{i j}^{t u b}$

$$
\tau_{i j}^{t o t}=\tau_{i j}+\tau_{i j}^{t u b}
$$

The mean stress tensor is defined as

$$
\tau_{i j}=\mu\left(\frac{\partial u_{i}}{\partial x_{j}}+\frac{\partial u_{j}}{\partial x_{i}}-\frac{2}{3} \frac{\partial u_{k}}{\partial x_{k}} \delta_{i j}\right)
$$

The relaxation time is used to characterize the time for a particle to settle in the new balance of the forces. Here, the particle momentum relaxation time, $\tau_{p}=\frac{\rho_{p} d^{2}}{18 \rho_{g} v}$, is the timescale on which a particle adapts to the surrounding flow conditions. The characteristic time of the fluid phase $\tau_{f}$ is calculated by $\tau_{f}=D / U_{i n j}$. The ratio of $\tau_{p}$ and $\tau_{f}$ is denoted as particle Stokes number,

$$
S t_{p}=\frac{\tau_{p}}{\tau_{f}}
$$

which characterizes the behavior of suspended particles. ${ }^{68}$

Particles with $S t \gg 1$ produce turbulence, while particles with $S t_{p} \ll 1$ completely follow oscillations of the gas velocity and dissipate turbulence. ${ }^{69}$ In this work, the particle Stokes number satisfies $S t_{p}<2.5$, and most of the droplets are small and their interactions with the turbulence are strong. Under these conditions, RANS modeling may fail in predicting the droplet motion and dispersion because of the restrictions of RANS modeling. ${ }^{37}$ Thus for the turbulence modeling in this chapter, we considered not only the RANS but also the LES approaches. 
In the RANS, the mean turbulent stress tensor, $\tau^{t u b}$, is the mean Reynolds-stress tensor in Eq. (2.20) determined by the RNG $k-\varepsilon$ model. The spray source terms in the turbulence model equations are discussed in Applendix B and Ref. ${ }^{48}$

In LES, the mean turbulent stress tensor is the residual stress in Eq. (2.37) for the Smagorinsky model, or Eq. (2.39) for the one equation model. In this study, the flow type is a dispersed multiphase flow, and the coefficient $C_{\mu}$ in Eq. (2.41) has been adjusted to $C_{\mu}=1.2$.

\subsection{Test conditions}

The geometry of the experiments resembles a cylinder with radius $r=55 \mathrm{~mm}$ and length $L=100 \mathrm{~mm}$, with the injector nozzle located on the top center (Fig. 4.1). A single-orifice, solid-cone injector is used for the droplet injection. All boundaries of the computation domain are set as non-slip walls. The gas in the cylinder is initially at rest. The fuel is injected into the cylinder with a common rail system at a constant injection pressure. Simulations have been performed for different ambient pressures of $p_{g}=1.1,3.0$ and 5.0 MPa. The experimental data for the non-evaporating cases are from Hiroyasa and $\mathrm{Kadota}^{70}$ and the evaporating data is from $\operatorname{Ko} \beta .^{71}$ The experimental settings are summarized in Table 4.1 .

Note that in the remaining part of the thesis, the non-evaporating cases are abbreviated as "cold" and the evaporating case as "evap". 
Table 4.1

Experimental settings

\begin{tabular}{ccc}
\hline & $\begin{array}{c}\text { Non-evaporating Case 1,2,3 } \\
{\left[\text { Hiroyasu } 1974^{70}\right]}\end{array}$ & $\begin{array}{c}\text { Evaporating } \\
{\left[\text { Ko } \beta 1989^{71}\right]}\end{array}$ \\
\hline$D[\mathrm{~mm}]$ & 0.3 & 0.2 \\
Gas type & air & $N_{2}$ \\
$p_{g}[\mathrm{MPa}]$ & $1.1,3.0,5.0$ & 5.0 \\
$T_{g}[\mathrm{~K}]$ & 300 & 800 \\
Fuel type & $\mathrm{DF} 2$ & HEP \\
$p_{\text {inj }}[\mathrm{MPa}]$ & 10 & 44.5 \\
$\dot{m}[\mathrm{~g} / \mathrm{s}]$ & $6.06,5.36,5.13$ & 4.62 \\
$\Delta t_{\text {inj }}[\mathrm{ms}]$ & $2.5,4.0,5.0$ & 1.3 \\
$\theta[\mathrm{deg}]$ & $20,23,25$ & 25 \\
\hline
\end{tabular}

The computational meshes are structured hexahedral, polar meshes. The cell density in the RANS meshes are concentrated radially and axially around the nozzle exit. The standard mesh in the RANS simulations has $28 \times 32 \times 36$ cells in radial, azimuthal and axial direction, with the smallest cell at the top center of the cylinder having a height of $1.0 \mathrm{~mm}$ and a radius of $1.0 \mathrm{~mm}$. The meshes used for the LES computations have a uniform cell distribution; the standard mesh has 76x76x140 cells, which corresponds to radial and axial cell sizes of approximately $0.7 \mathrm{~mm}$.

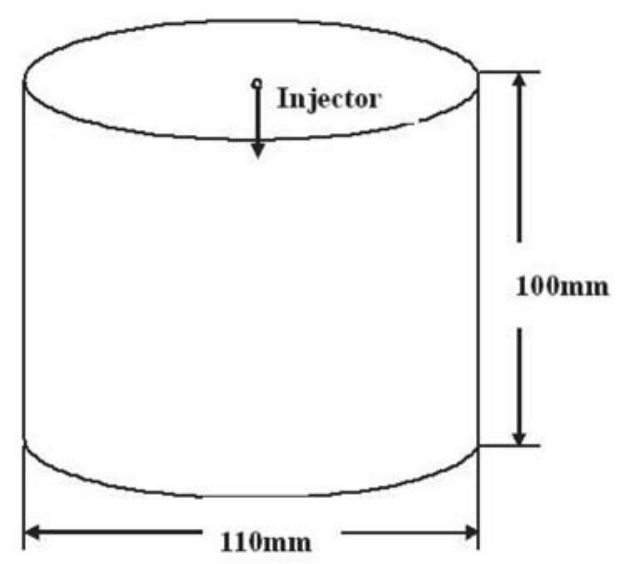

Figure 4.1: Computation domain. 


\subsection{LES/RANS results and discussion}

\subsubsection{Non-evaporating sprays}

\section{Mesh sensitivity study}

Mesh sensitivity studies have been conducted for the RANS and the LES approaches using diesel fuel. In both cases, the number of cells used in the standard meshes have been varied by a factor of 1.5 in each direction. For the RANS, this lead to the coarse mesh of $18 \times 32 \times 24$ cells and a fine mesh of $42 \times 32 \times 54$, whereas for the LES approach, the coarse mesh has 55x76x100 cells and the fine mesh 110x76x200 cells.

The results of this mesh sensitivity study are shown in terms of the spray tip penetration in Figs. 4.2 and 4.3. The penetration has been obtained by including $99 \%$ of the entire spray mass, that is, $1 \%$ of the droplets that are farthest away from the nozzle have been omitted.

For the RANS simulations shown in Fig. 4.2, the spray tip penetration of the three meshes are nearly the same, which demonstrates mesh independence. In fact, these values agree with the values in Ref. ${ }^{51}$

For the LES simulations shown in Fig. 4.3, the penetration increases with the number of the cells. However, the difference between the fine mesh and the standard mesh is much smaller than the difference between the standard mesh and the coarse mesh, which is a clear indication of mesh convergence. 


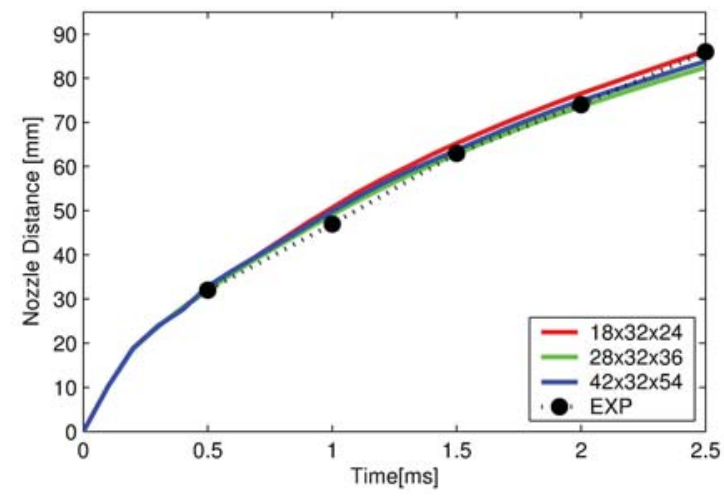

Figure 4.2: Temporal change in spray tip penetration with different grid size for RANS simulation.

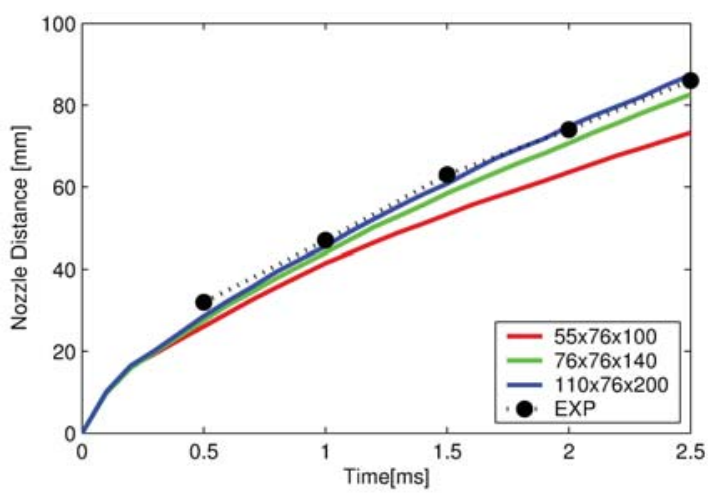

Figure 4.3: Temporal change in spray tip penetration with different grid size for LES simulation.

Note that, in opposition to the RANS simulations, the LES simulations are not mesh independent. Theoretically, a very dense LES can approach the DNS accuracy when the subgrid energy can be neglected. In practice, the subgrid models, designed for a certain grid size range, are flawed in the cells whose grid size is nearly zero. Pope ${ }^{72}$ found that the constant $C_{S}$ in Eq. (2.38) of the Smagorinsky model does need adjustment in the region far from the optimal inertial range of the turbulent spectrum. ${ }^{72}$ Here, the constant $C_{\mu}$ in Eq. (2.41) of the LES one equation model is also tuned to compensate for the modeled subgrid energy ${ }^{73}$ such that the penetration curve predicted by means of the LES standard mesh is near to the experimental result.

Additionally, the computation time of Cold Case 1 with different mesh resolutions are shown in Table 4.2. Note that in the LES cases, the actual CPU time are approximately four times the running CPU time, since the LES calculations was done in parallel using four processors. Obviously, LES simulations are much more time consuming than RANS. 
Table 4.2

Computation Time of Cold Case 1

\begin{tabular}{cccc}
\hline RANS & $18 \times 32 \times 24$ & $28 \times 32 \times 36$ & $42 \times 32 \times 54$ \\
\hline Num. of cells & 13824 & 32256 & 72576 \\
CPU time [s] & 1514 & 4044 & 7900 \\
CPU time per cell [s] & 0.110 & 0.125 & 0.109 \\
\hline LES & $55 \times 76 \times 100$ & $76 \times 76 \times 140$ & $110 \times 76 \times 200$ \\
\hline Num. of cells & 418000 & 808640 & 1672000 \\
CPU time [s] & 119428 & 246644 & 681244 \\
CPU time per cell [s] & 0.286 & 0.305 & 0.407 \\
\hline
\end{tabular}

First, LES requires a finer mesh resolution, and the increased number of cells consume much more computation time. Second, the average CPU time per cell for LES cases are also increasing with the number of cells, while the corresponding values in the RANS cases are around 0.1 second. This may be caused by the CPU time required for the data transfer between the different processors for parallel calculation. Hence, though the LES has advantages in predicting spray phenomena, the time saving RANS is still widely used.

\subsubsection{Spray tip penetration of Cases 1-3}

The spray tip penetration is the most basic characterisation of a spray. Computationally, the liquid penetration is determined as the maximum distance between the nozzle and the center of the cell where the integrated liquid mass is $90 \%$ of the total mass.

In Fig. 4.4, the penetrations of the computation Cases 1-3, using diesel fuel, are compared with the experimental results of Hiroyasu and Kadota. ${ }^{70}$ This figure shows that the penetration curves predicted by LES are near to the ones of the RANS, and both the RANS and LES results are close to the experimental data. In Case 2, the LES result is nearly the 


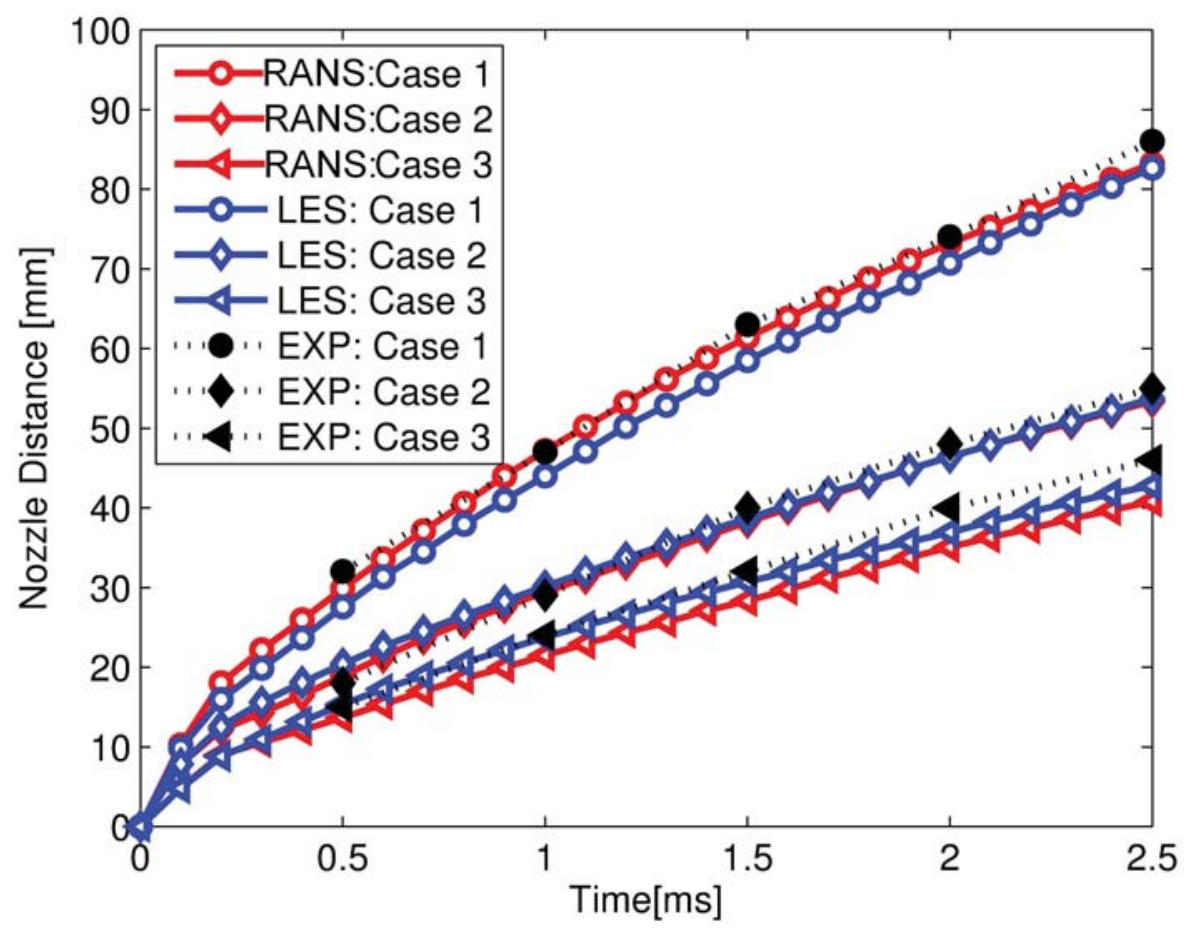

Figure 4.4: Temporal change in spray tip penetration for RANS and LES using DF2.

same as the RANS. In Case 1, compared with the RANS result, the LES penetration curve is a little farther away from the experimental result, while the LES result in Case 3 is better than the RANS.

\subsubsection{Comparison between RANS and LES}

Figures 4.5 and 4.6 show the RANS and LES gas phase velocity vectors at the injection time of $0.0024 \mathrm{~s}$ for non-evaporating Case 1 . The RANS shows a parabolic velocity distribution, while the LES case exhibits a random velocity field at the outer edge of the spray. In the RANS case, although a stochastic dispersion model is applied to add randomness to the droplet motion, the RANS still fails to disperse the spray droplets sufficiently. In contrast, the LES case does not use a dispersion model, but it captures the SGS turbulence. 


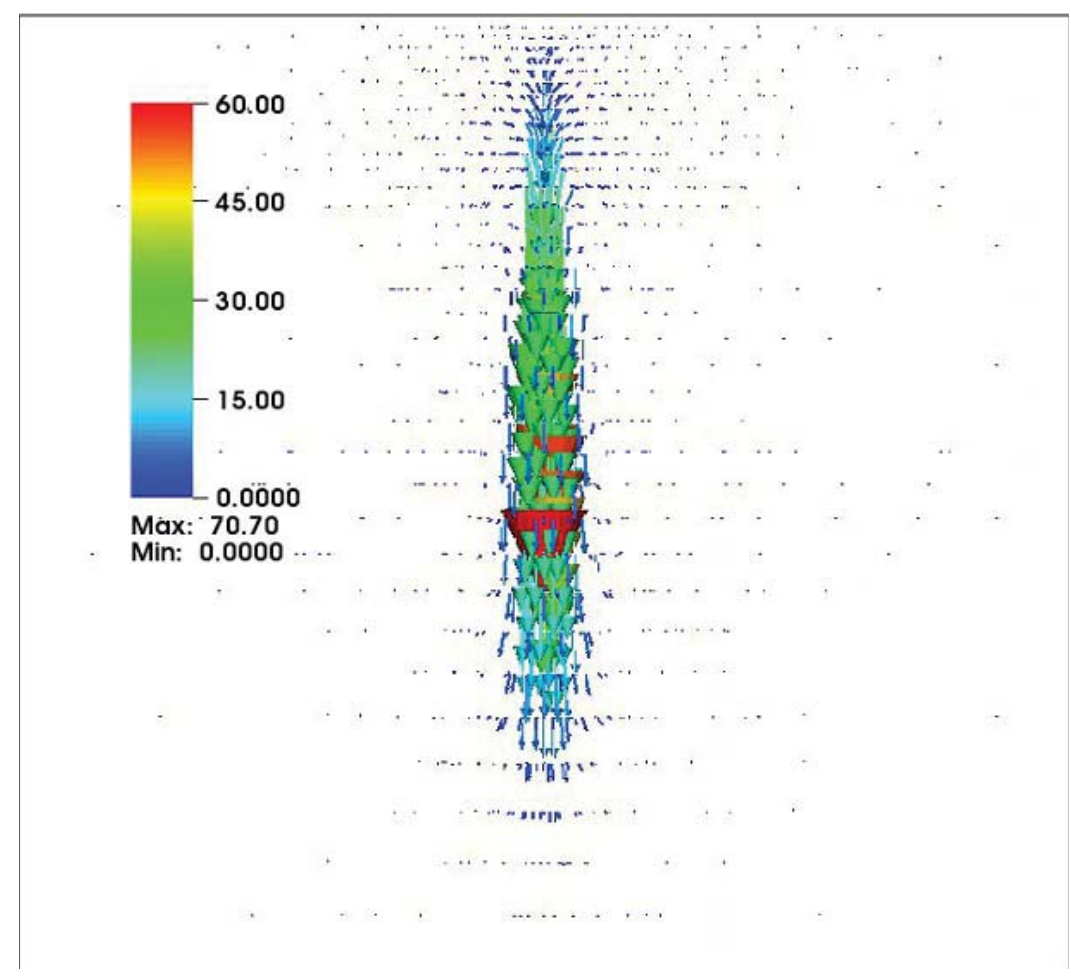

Figure 4.5: Gas phase velocity vector at cross section for RANS Case 1 at time $0.0024 \mathrm{~s}$.

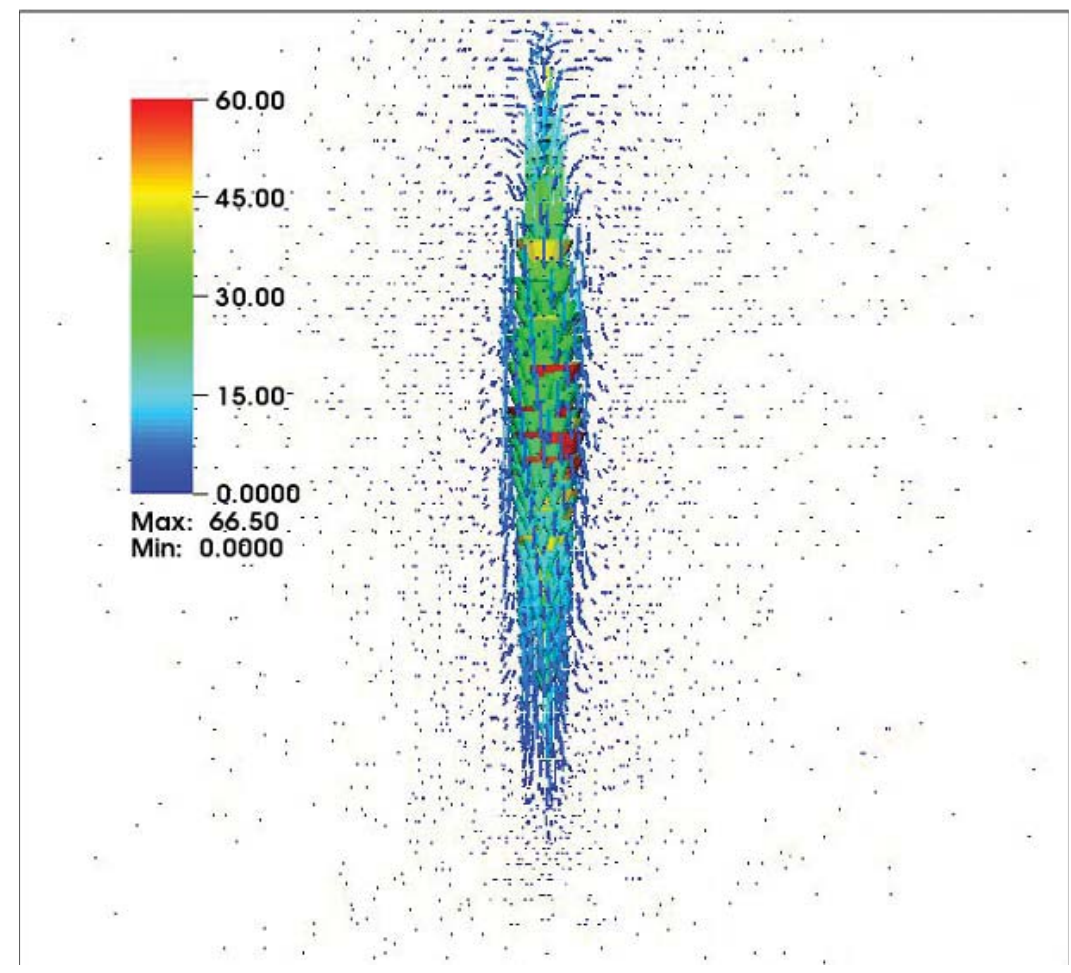

Figure 4.6: Gas phase velocity vector at cross section for LES Case 1 at time $0.0024 \mathrm{~s}$. 


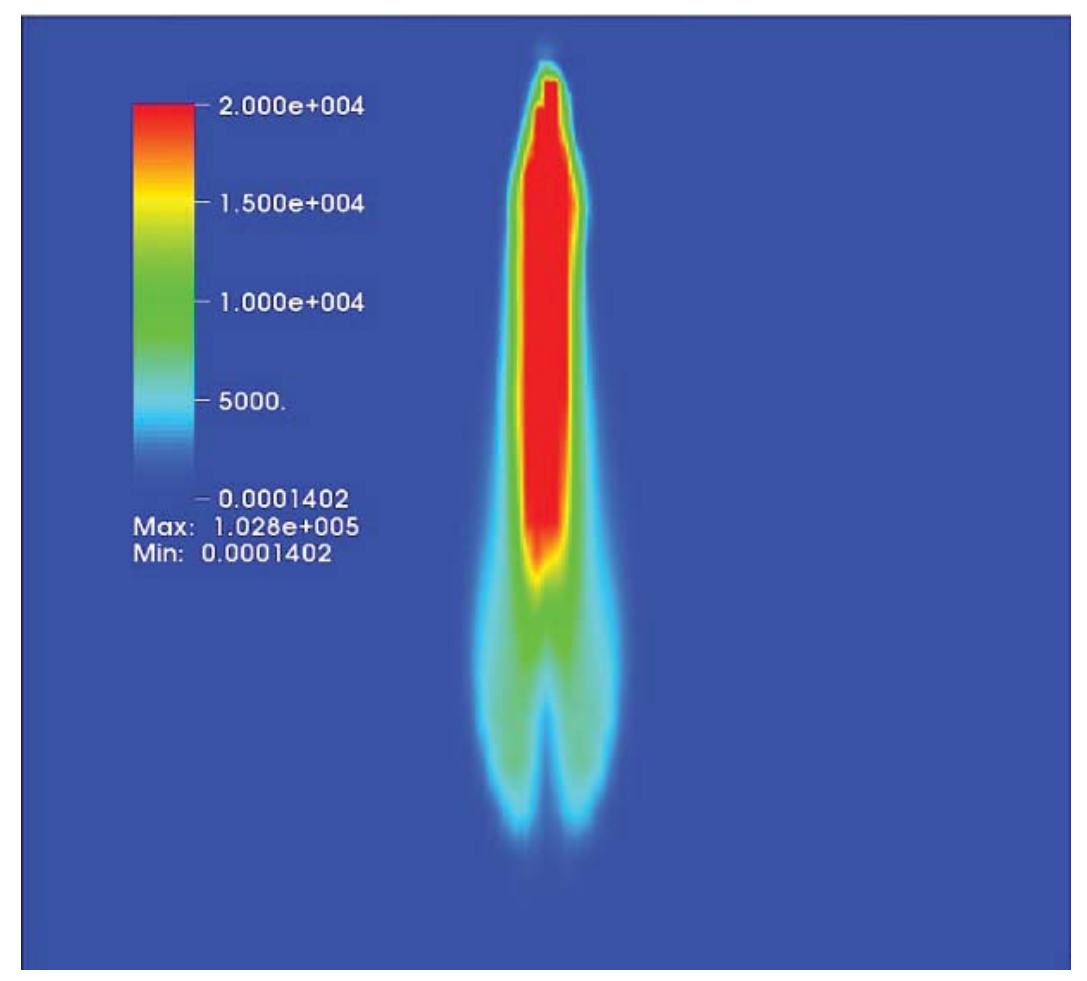

Figure 4.7: Cross-sectional plot for RANS Case 1 colored by magnitude of vorticity at time $0.0024 \mathrm{~s}$.

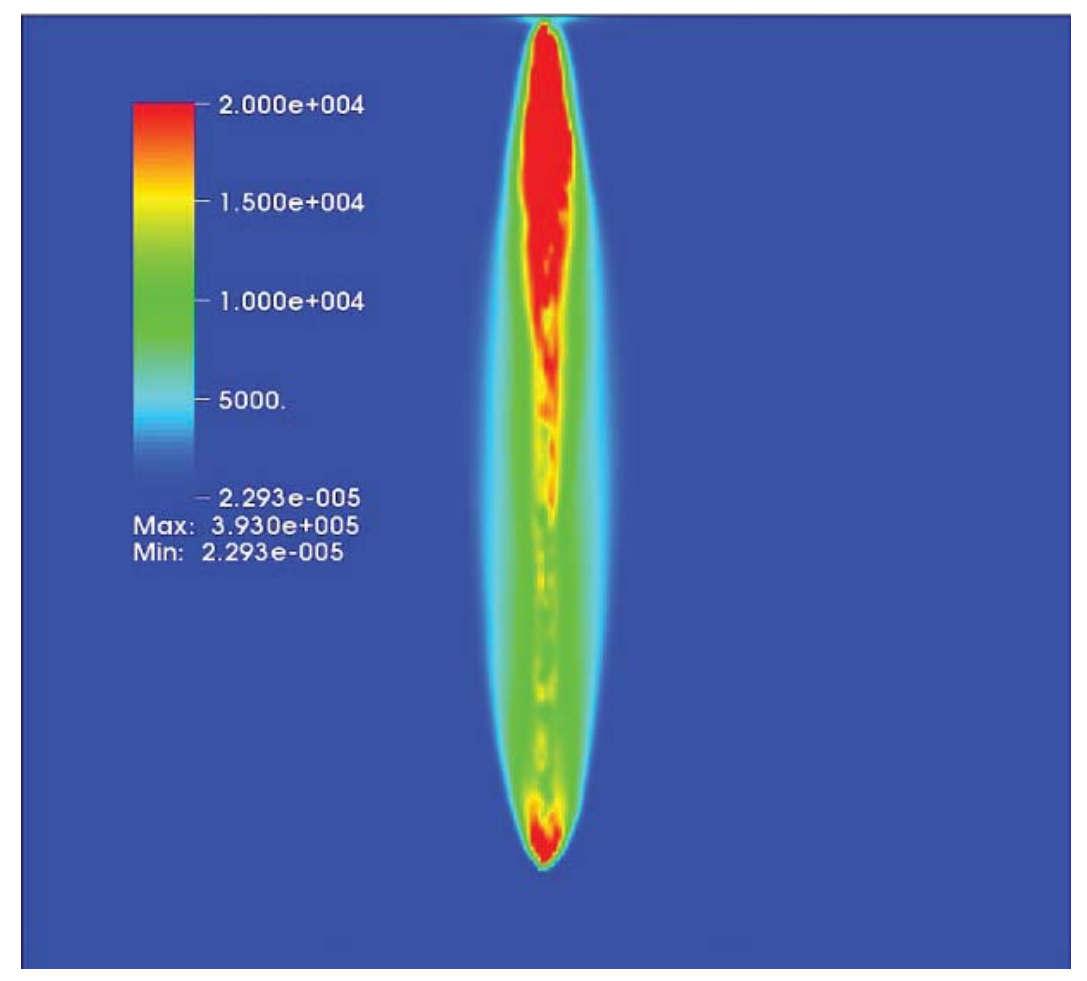

Figure 4.8: Cross-sectional plot for LES Case 1 colored by magnitude of vorticity at time $0.0024 \mathrm{~s}$. 

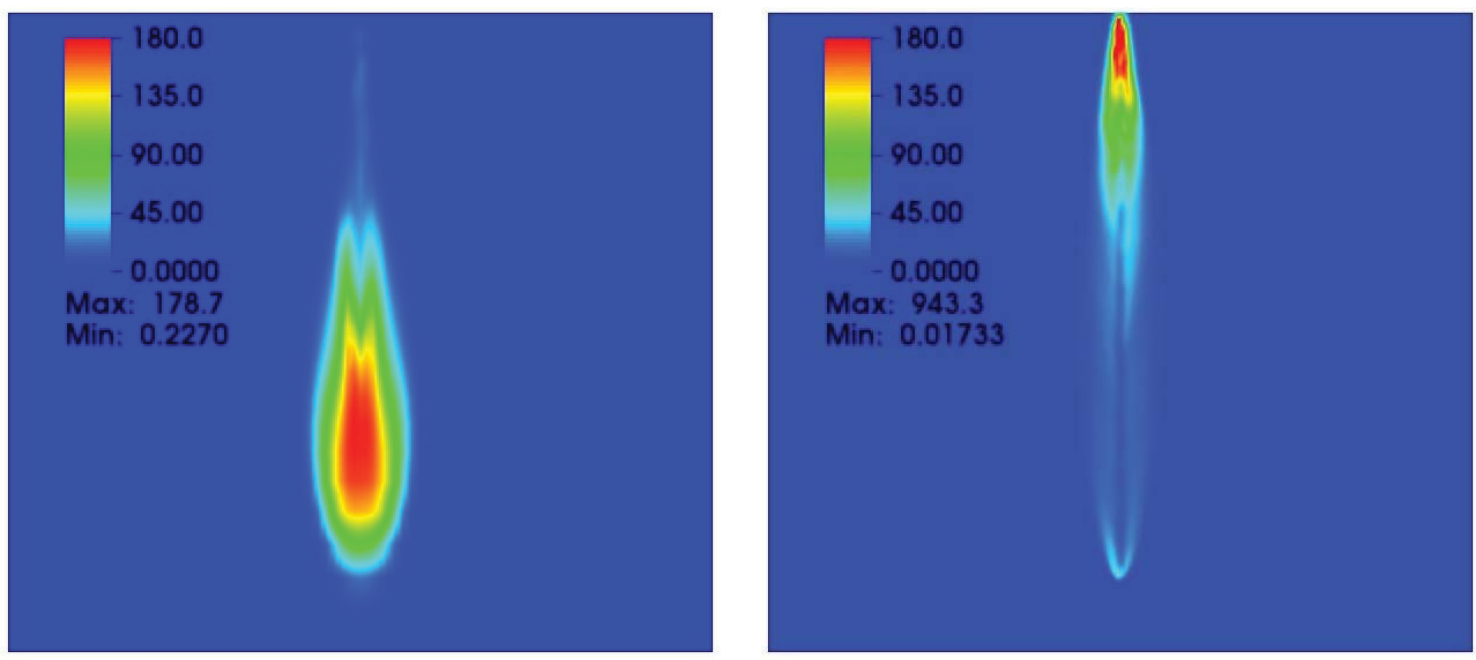

Figure 4.9: Cross-sectional plot for RANS Case 1 colored by turbulent kinetic energy at time $0.0024 \mathrm{~s}$.
Figure 4.10: Cross-sectional plot for LES Case 1 colored by turbulent kinetic energy at time $0.0024 \mathrm{~s}$.
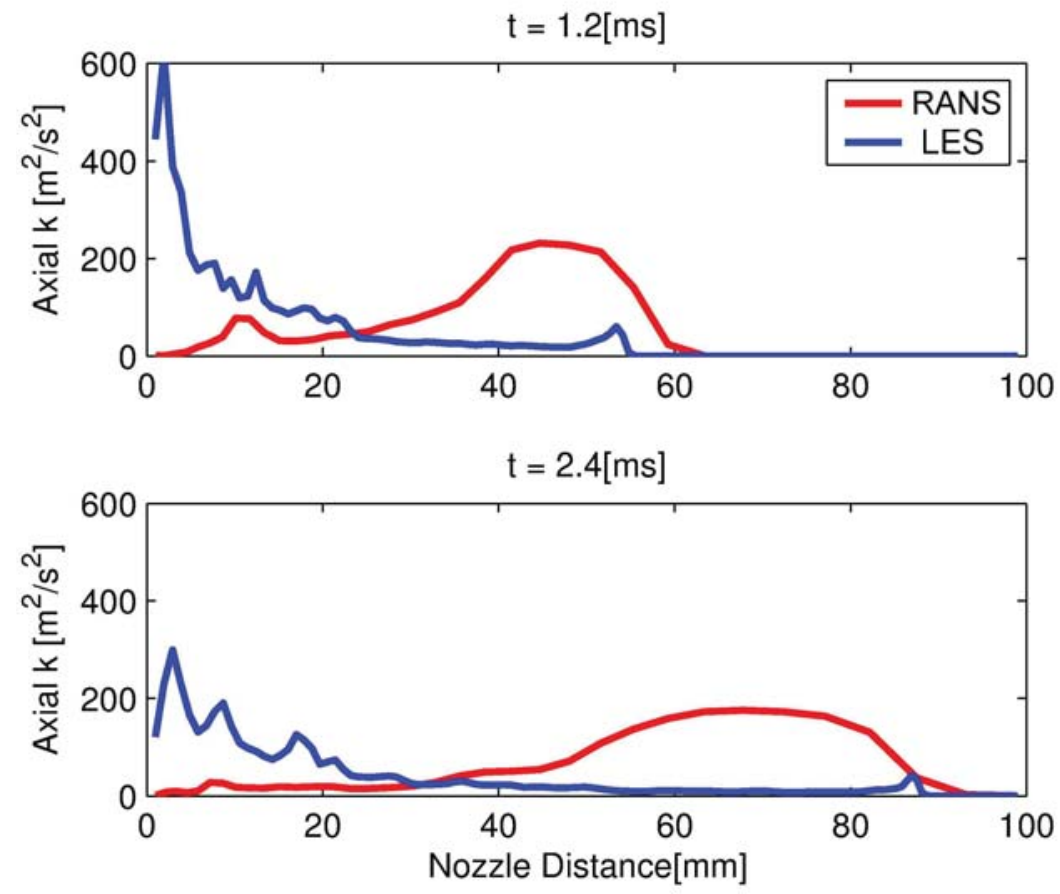

Figure 4.11: Turbulent kinetic energy $k$ versus nozzle distance along centerline of Case 1. 
The magnitude of the gas phase vorticity, $\|\omega\|=\|\nabla \times \mathbf{u}\|$, is shown in Figs. 4.7 and 4.8 for the RANS and the LES, respectively. As can be seen, in the LES case, the vorticity is spread much more evenly throughout the spray, including at the spray tip. In contrast, the RANS simulation does not show any significant vorticity at the spray tip. Note vorticity at the spray tip is expected, since the interaction of droplets with the undisturbed gas is likely to induce vorticity.

Additionally, the RANS failed to capture the vorticities produced near the nozzle exit. This is consistent with the observations in Fig. 4.5. Towards the end of injection, near the nozzle exit, the gas flow velocity is small in magnitude, but disturbed by the surrounding droplets and flow oscillations. The resulting vorticity is captured by the LES and shown in Figs. 4.6 and 4.8.

The turbulent kinetic energy for the RANS and the LES are shown in Figs. 4.9 and 4.10. In the RANS simulations, the largest turbulent kinetic energy is observed towards the tip of the spray, whereas in the LES case, it is closer to the nozzle exit. In RANS, the $k$ equation, Eq. (2.27), models the transport of the turbulent kinetic energy, which reflects the whole flow field. In LES, the $k^{s g s}$ equation, Eq. (2.40), resolves the SGS turbulent kinetic energy, which only contributes a portion of the overall turbulence. Because the small scale eddies are more uniform than the large scales, LES modeling of subgrid energy is less challenging and expected to be more reasonable.

Details of the turbulent kinetic energy along the spray axis are shown in Fig. 4.11 at time 
$t=0.0012 s$ and $t=0.0024 s$. In the RANS case, the peak value of the axial turbulent kinetic energy appears to be moving downstream with the spray, while the peak value predicted by LES is closer to the nozzle orifice. Note that the LES simulations predict a small peak in the turbulent kinetic energy near the tip of the spray; this is consistent with the tip vorticity observed in Fig. 4.8.
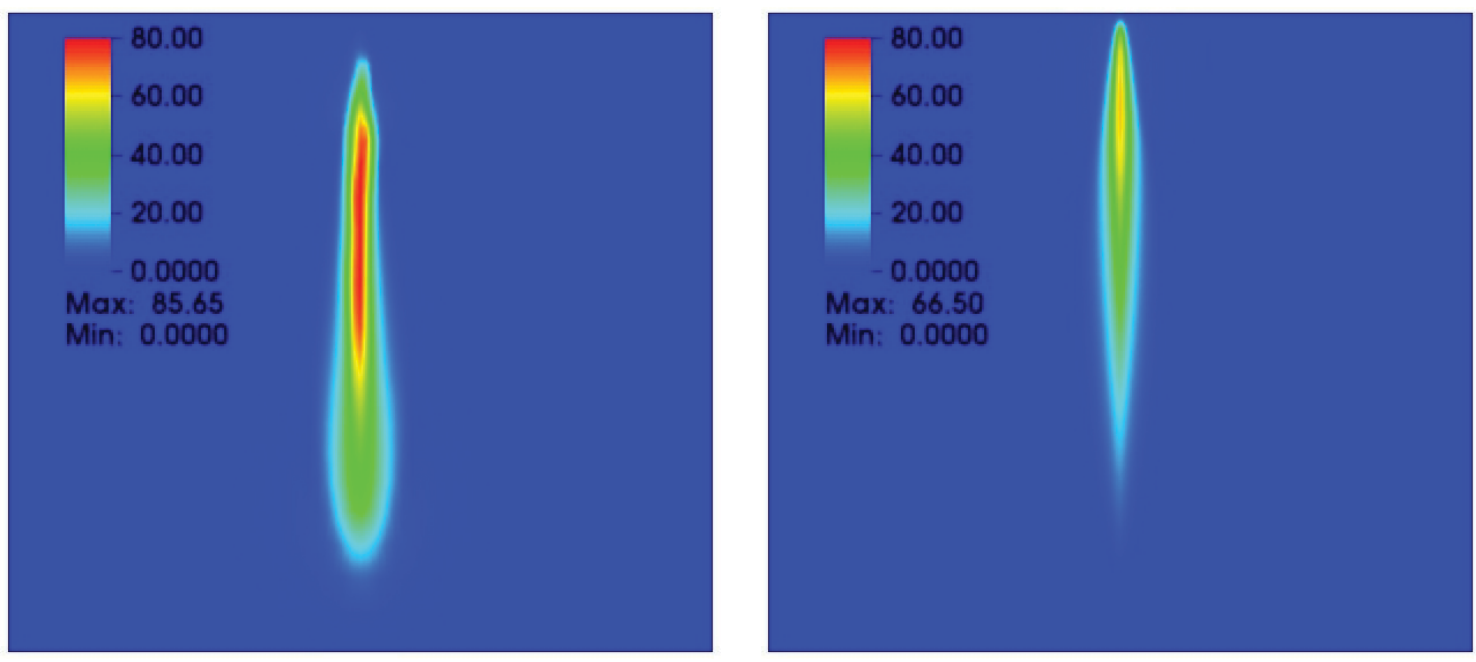

Figure 4.12: Cross-sectional plot for RANS Case 1 colored by the magnitude of the fluid velocity at time $0.0024 \mathrm{~s}$.

Figure 4.13: Cross-sectional plot for LES Case 1 colored by the magnitude of the fluid velocity at time $0.0024 \mathrm{~s}$.
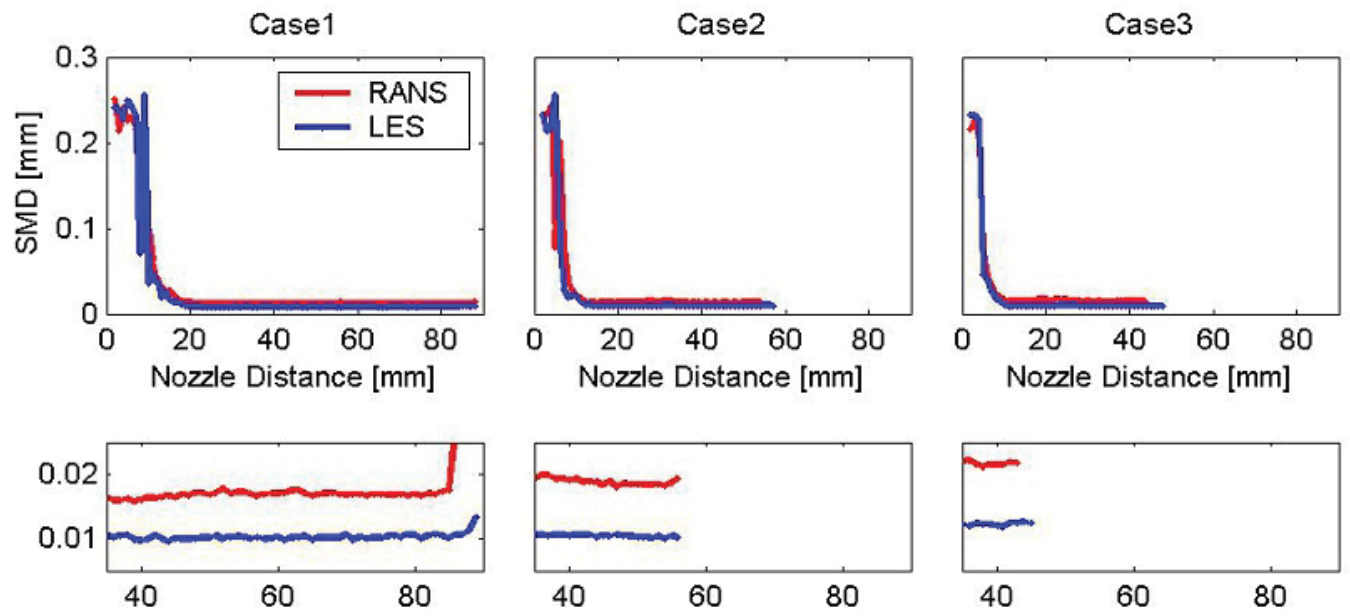

Figure 4.14: Cross-sectional SMD at time $0.0024 \mathrm{~s}$ for Case 1 . 
The RANS and LES simulations exhibit big differences in the prediction of the small scale eddies, which in turn has a large influence on the prediction of the vorticity and the turbulent kinetic energy. The large scale quantities, e.g. the flow velocity, predicted by these two methods also differ significantly. This is because of the coupling of the small scales with the larger scales via the Kolmogrov dissipation cascade, illustrated by the cross sectional plot of the magnitude of the averaged velocity in Fig. 4.12 and Fig. 4.13.

On the other hand, the breakup of the small droplet is mainly influenced by the viscous shear related with the small eddies. ${ }^{74}$ The better prediction of the small eddies in the LES leads to the smaller SMDs near the spray tip, as shown in Fig. 4.14. This figure shows the Sauter mean diameter (SMD) versus nozzle distance of Cases 1-3 at the injection time of $0.0024 \mathrm{~s}$. The plots in the second row show the details of the curves near their minimal values. As is seen, the droplet size decreases rapidly near the nozzle exit at a distance of less than $10 \mathrm{~mm}$, and may increases gradually towards the spray tip because of droplet coalescence caused by droplet collisions.

More details of droplet size are shown in Figs. 4.15 and 4.16, which show the droplet diameters at the injection time of 0.0024 s. Obviously, the RANS case predicted larger drop diameters than the LES, especially near the nozzle exit. Moreover, the RANS develops a much wider spray than the LES. For the RANS simulations, the small scale eddies are not sufficiently resolved, nor correctly predicted; it is not surprising that the dissipation rate is overpredicted. Therefore, the resulting over-prediction of the jet spreading is indicated by 
the wider fuel rich regions as shown in Fig. 4.15.

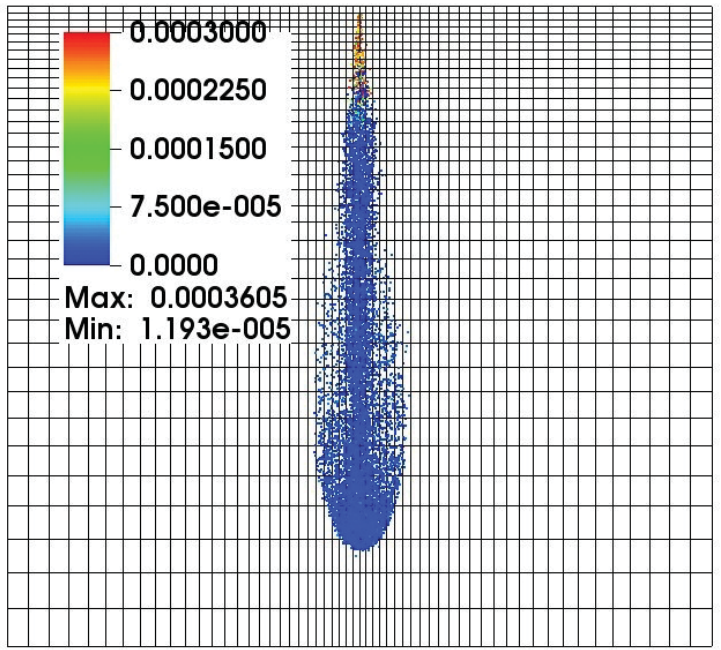

Figure 4.15: Spray plot for RANS Case 1 colored by droplet diameter at time $0.0024 \mathrm{~s}$.

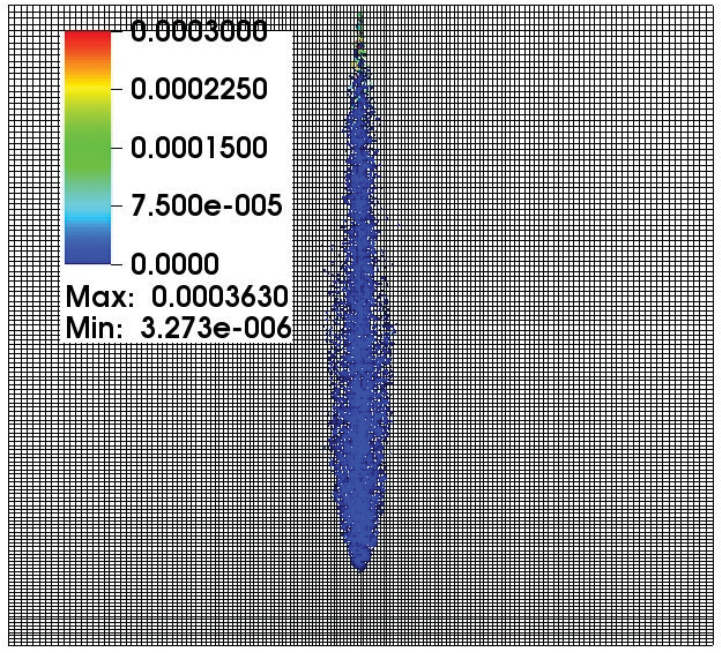

Figure 4.16: Spray plot for LES Case 1 colored by droplet diameter at time $0.0024 \mathrm{~s}$.

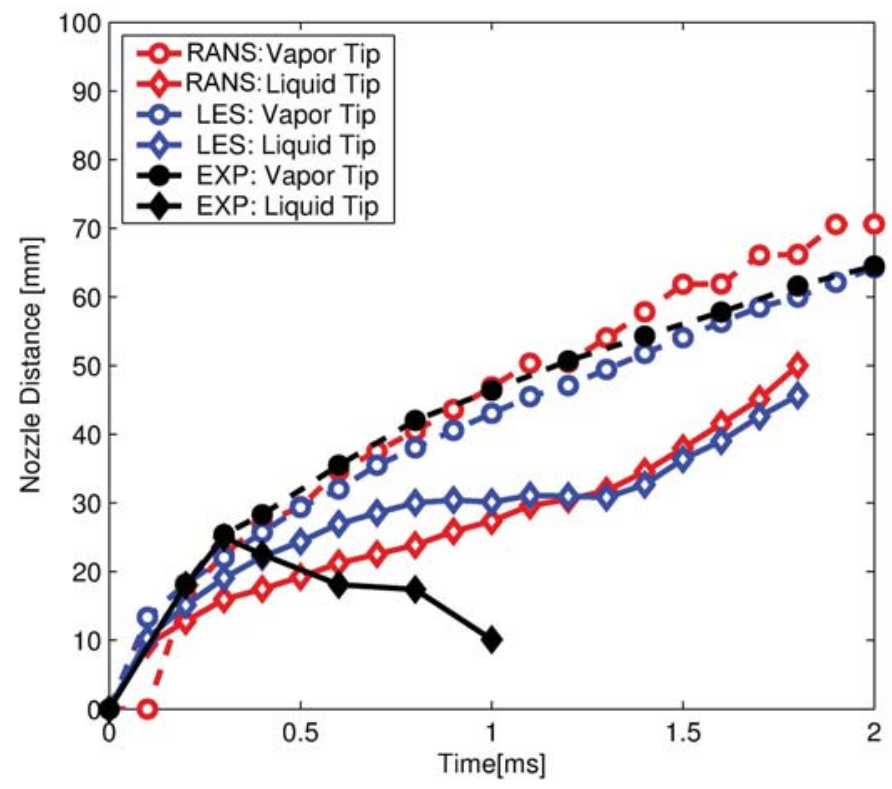

Figure 4.17: Temporal change in spray tip penetration for RANS and LES evaporating case using HEP in evaporating case. 


\subsubsection{Evaporating sprays}

For evaporating sprays, n-heptane is injected into the spray chamber instead of diesel fuel \#2. The comparison of RANS and LES are done similar as for the non-evaporating cases.

\section{Spray tip penetration}

In Fig. 4.17, the penetrations of the evaporating sprays are compared with the experimental results of $\operatorname{Ko} \beta .^{71}$ The vapor penetration is determined as the maximum distance between the nozzle and the center of the cells for which the fuel vapor mass fraction is 0.067 , i.e., the stoichiometric fuel/air ratio. ${ }^{51}$ The vapor penetration curve predicted by LES lies closer to the experimental points than RANS. Note that the ragged look of the RANS simulated vapor penetration curve is due to the spatial discretization of the computational mesh. This problem is solved in the LES simulation because a finer mesh is used.

In contrast to the vapor penetration, the liquid penetration of n-heptane is significantly over predicted by both RANS and LES for $t \geq 0.5 \mathrm{~ms}$. This over prediction can be explained by the fact, that the experimental data has been obtained from shadow graphs, while the simulation penetrations have been obtained by averaging the droplets at the tip of the spray. Since isolated droplets at the spray tip may go undetected by shadow graph methods, it is not surprising that the simulation penetrations are over predictions. Therefore, considering the different approaches in determining the liquid penetrations, the agreement in liquid penetration between simulation and experiment can be considered acceptable. 


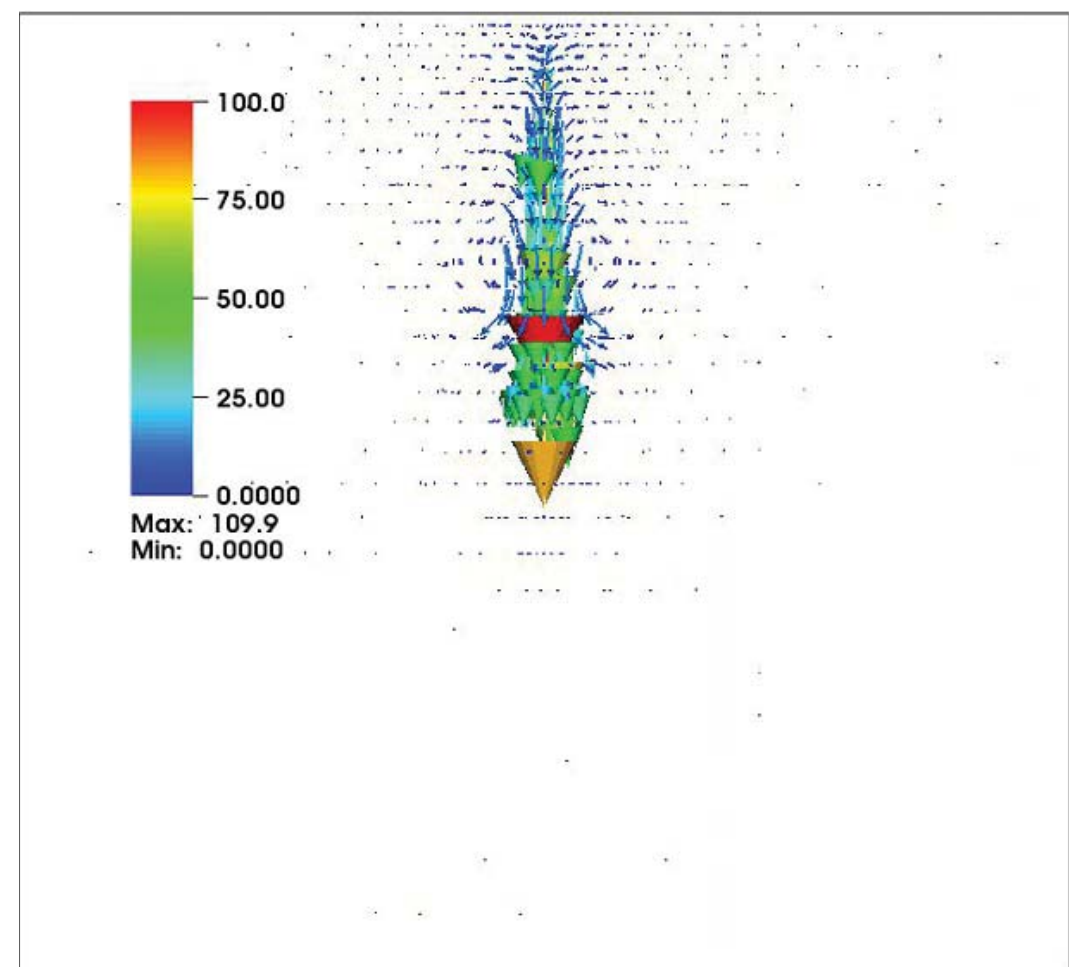

Figure 4.18: Gas phase velocity vector at cross section for RANS evaporating case at time $0.0008 \mathrm{~s}$.

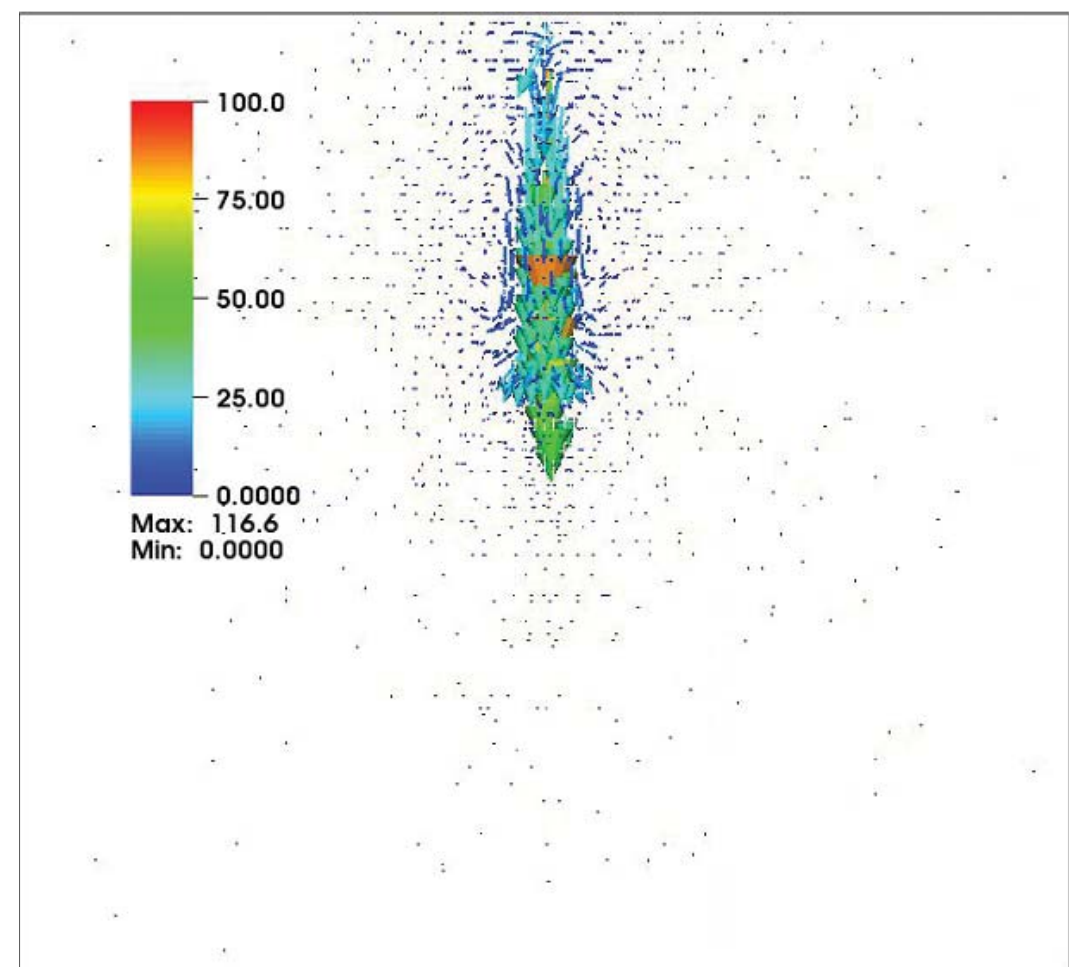

Figure 4.19: Gas phase velocity vector at cross section for LES evaporating case at time $0.0008 \mathrm{~s}$. 


\section{Comparison between RANS and LES}

The vector plots of the gas flow velocity at the injection time of $0.0008 \mathrm{~s}$ are shown in Fig. 4.18 for RANS and Fig. 4.19 for LES. As for the non-evaporating sprays, the vector plots also show that the LES captures more turbulent fluctuation velocity than RANS. The RANS still shows a parabolic velocity distribution, while the LES case captures much more random velocity fluctuations at the outer edge of the spray.
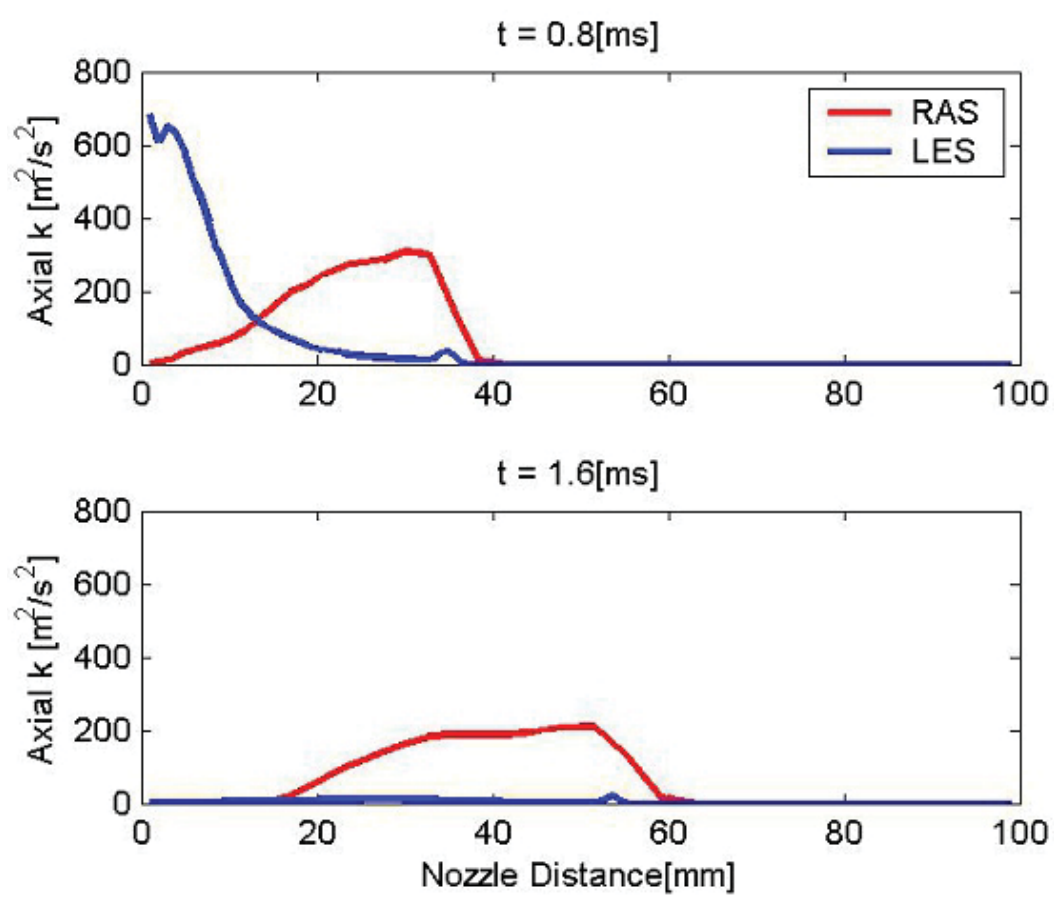

Figure 4.20: Turbulent kinetic energy $k$ versus nozzle distance along centerline of evaporating case.

Fig. 4.20 shows the turbulent kinetic energy along the spray axis at time $=0.0008 \mathrm{~s}$ and

$0.0016 \mathrm{~s}$. Similar to the non-evaporating cases, the peak value predicted by LES at time $=0.0008 \mathrm{~s}$ is closer to the nozzle orifice than RANS, and a dramatic decrease of the peak 


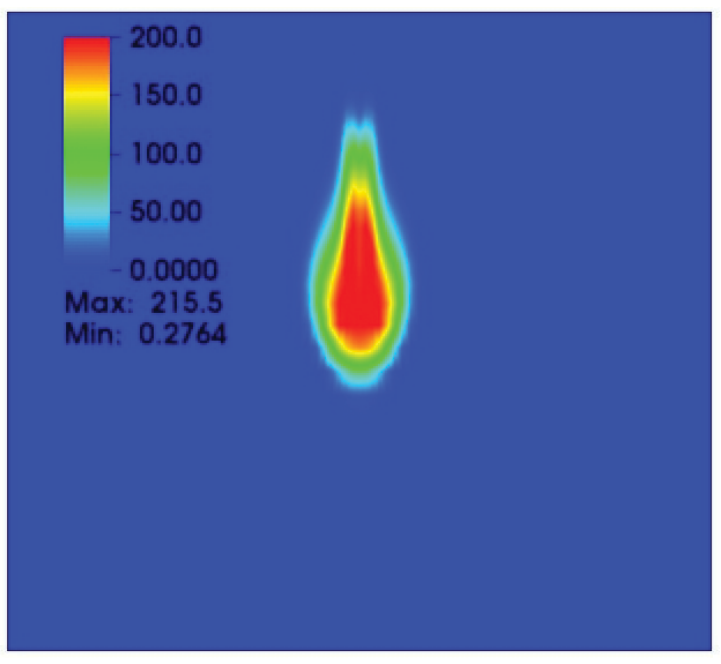

Figure 4.21: Cross-sectional plot for RANS evaporating case colored by turbulent kinetic energy at time $0.0016 \mathrm{~s}$.

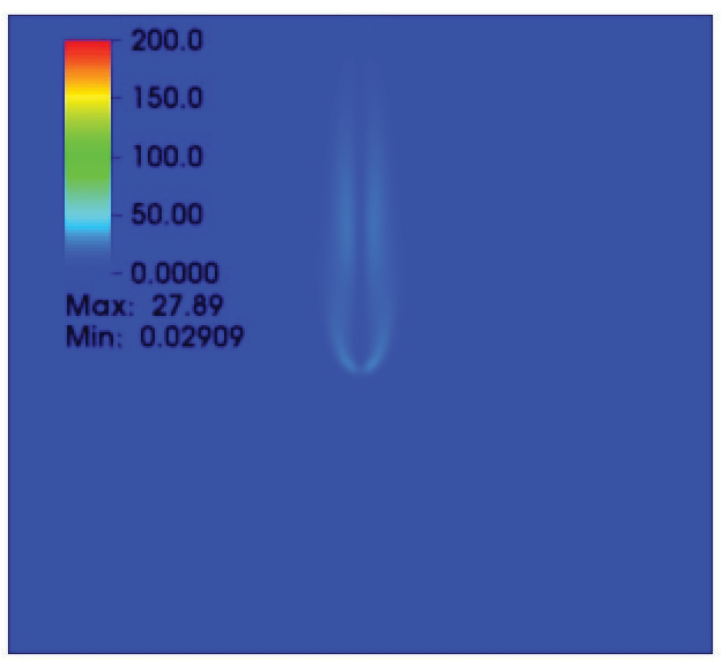

Figure 4.22: Cross-sectional plot for LES evaporating case colored by turbulent kinetic energy at time $0.0016 \mathrm{~s}$.
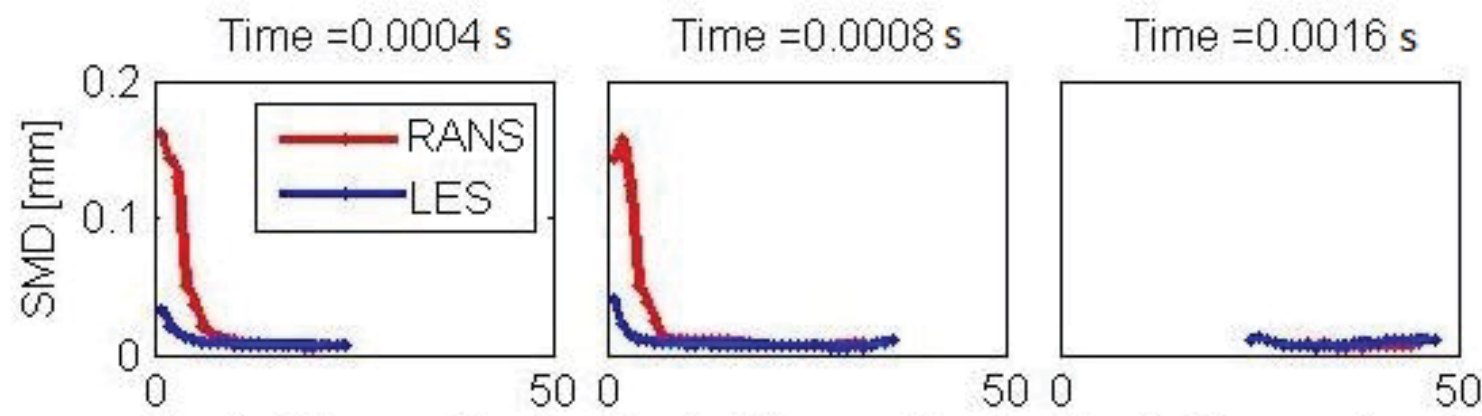

Nozzle Distance [mm]

Nozzle Distance [mm]

Nozzle Distance [mm]
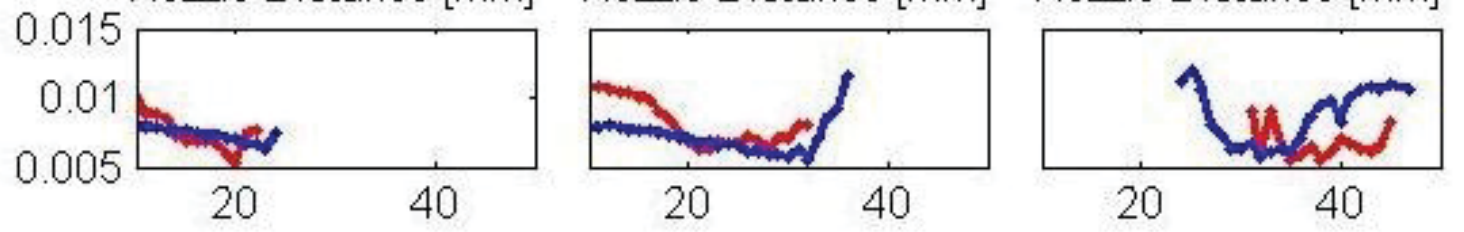

Figure 4.23: Cross-sectional $S M R$ at time $=0.0016 \mathrm{~s}$ for evaporating case.

value to zero is also observed in LES. Additionally, again for the evaporating case, the LES simulations predict a small peak in the turbulent kinetic energy near the tip of the spray. More details of the turbulent kinetic energy at the injection time $0.0016 \mathrm{~s}$ can be observed in Figs. 4.21 and 4.22. 
Fig. 4.23 shows the Sauter mean diameter (SMD) versus nozzle distance of the evaporating case at the injection time of $0.0004 \mathrm{~s}, 0.0008 \mathrm{~s}$ and $0.0016 \mathrm{~s}$. The plots in the second row show the details of the curves near their minimal values. As for the non-evaporating Case 1, the droplet size decreases rapidly near the nozzle exit at a distance of less than $10 \mathrm{~mm}$. Also the droplet size increases gradually towards the spray tip because of droplet coalescence caused by droplet collisions.

Additionally, near the spray tip, most of the droplets are evaporated or nearly evaporated. Hence, the droplet sizes predicted by RANS and LES are similar near the spray tip. This is different from the non-evaporating cases. The evaporation process leads to the decrease of the spray tip SMDs, which is lower than the spray tip SMDs $(\approx 0.01 \mathrm{~mm})$ of the LES non-evaporating case in Fig. 4.14.

Another difference between RANS and LES for the evaporating case is that the droplet sizes predicted by RANS are much larger than those by LES near the nozzle. The breakup of the large droplet is caused mostly by the dynamic pressure force. ${ }^{74}$ As discussed in the non-evaporating cases, the vorticities near the nozzle exit, as shown in Fig. 4.19, imply a greater dynamic pressure force which accelerates the droplet breakup in the LES.

More details of droplet size are shown in Figs. $4.24-4.27$, which show the droplet diameters at the injection time of $0.0008 \mathrm{~s}$ and $0.0016 \mathrm{~s}$. From these figures, it is apparent that droplets calculated using LES evaporates faster than those using RANS. The smaller droplet size leads to a greater liquid-to-air surface ratio in the LES. Therefore, the heat 
exchange between the liquid and the gas is enhanced, which leads to the acceleration of the evaporation rate in the LES.

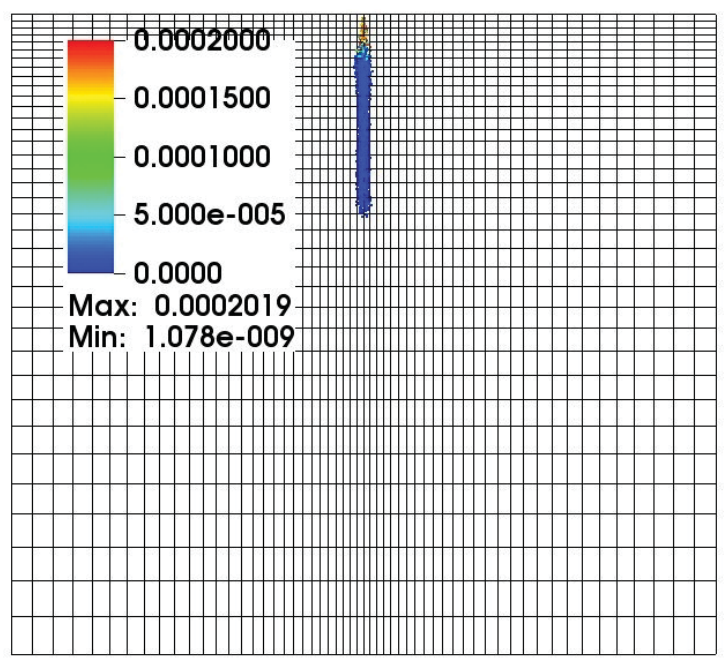

Figure 4.24: Spray plot for RANS evaporating case colored by droplet diameter at time $0.0008 \mathrm{~s}$.

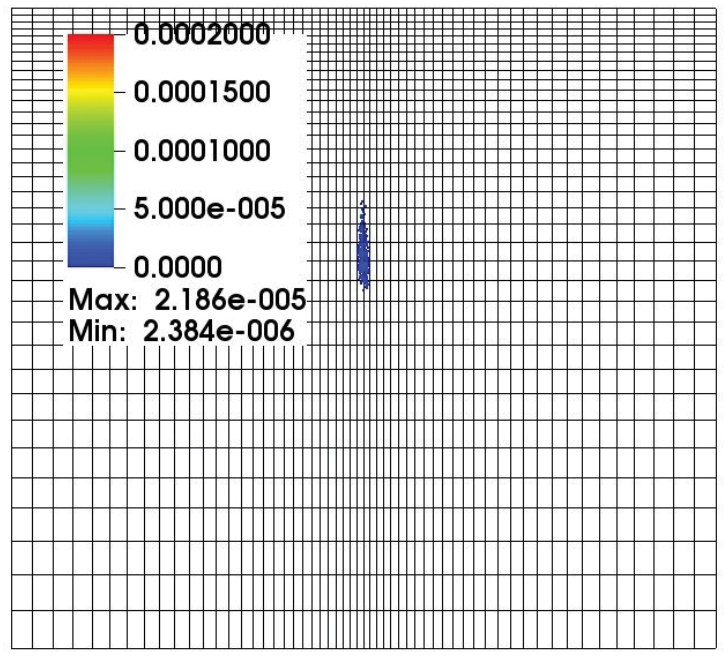

Figure 4.26: Spray plot for RANS evaporating case colored by droplet diameter at time $0.0016 \mathrm{~s}$.

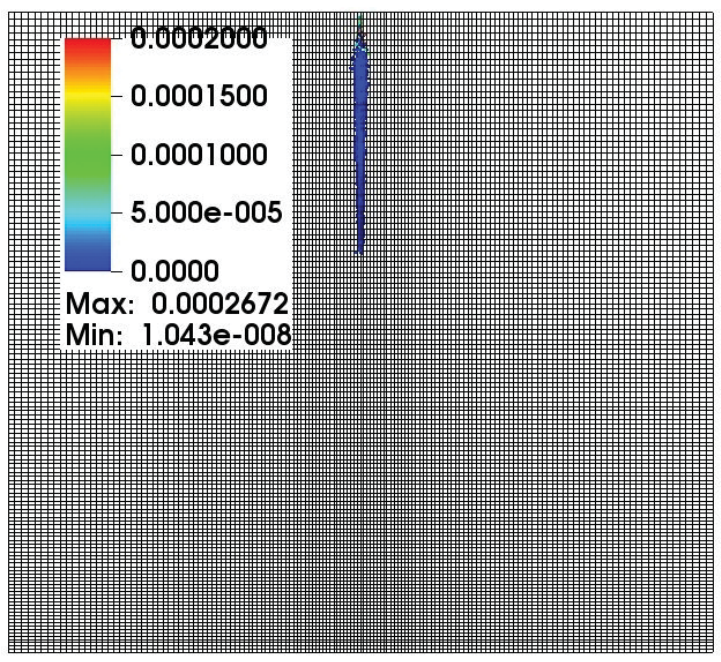

Figure 4.25: Spray plot for LES evaporating case colored by droplet diameter at time $0.0008 \mathrm{~s}$.

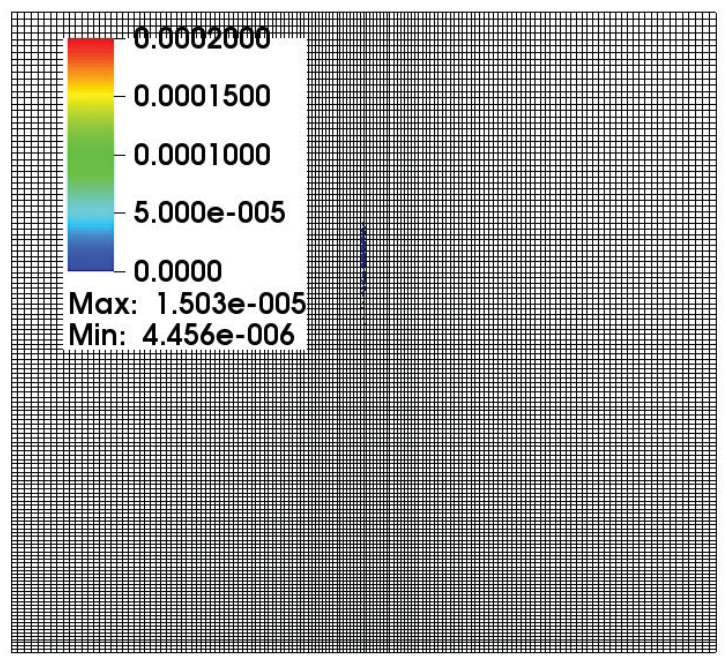

Figure 4.27: Spray plot for LES evaporating case colored by droplet diameter at time $0.0016 \mathrm{~s}$. 


\subsection{Conclusion}

Comparisons of RANS and LES have been performed by simulating non-evaporating sprays for diesel fuel and evaporating sprays for n-heptane.

The RANS simulation resolves the time averaged turbulence, while the LES simulation resolves the small and most important scales of the turbulence. The time-averaged NavierStokes equations and the spatial-filtered Navier-Stokies equations have the same form, and the constants in the spray submodels are also the same. The only difference is the mesh resolution and the turbulence modeling.

The RANS simulation needs to model the fluctuation, which is affected by all scales of the turbulence. This is much more difficult than to model the small scale turbulence in LES. Thus, quantities that are affected by the small scale eddies or the fluctuation part of the turbulence, e.g. the SGS turbulent kinetic energy, are predicted more accurately by the LES simulation.

Moreover, the existence of the unsteady vortical structures enhances the mixing of liquid fuel and the surrounding air. ${ }^{75}$ RANS failed to capture the unsteady vortical structures because of the time averaging of the governing equations. This is avoided in LES by taking spatial filtering, which leads to one of the advantages of LES in predicting the unsteady mixing. 
Finally, the insufficient prediction of the small scale eddies leads to the over-dissipation of the RANS. Then the resulting over-prediction of the jet spreading causes the wider shape of the RANS spray. 


\section{Hydrotreated vegetable oil (HVO) sprays}

Climate change is widely recognized as one of today's most serious issues. To contribute to the solution of this problem, the reduction of greenhouse gas emissions is believed to be a necessity. Alternative fuels with high cetane numbers and lack of aromatic components can make a significant contribution towards this end. One such fuel, hydrotreated vegetable oil (HVO), is a renewable synthetic diesel hydrocarbon fuel without sulfur, aromatics and

oxygenates (c.f. Refs. ${ }^{76-78}$ ). Consequently, HVO-powered diesel engines are expected to greatly reduce emissions such as carbon monoxides, nitrogen oxides, hydrocarbon and particulate matter.

Hydrotreated vegetable oil (HVO) is a high-cetane number alternative fuel with the potential of drastic emissions reductions in high-pressure diesel engines. In this chapter, the behavior of HVO sprays is investigated computationally and compared with conventional diesel fuel sprays. The turbulence has been modeled with the RNG $k-\varepsilon$ model for the RANS simulations and the one equation model for the LES simulations.

Simulations of non-evaporating and evaporating sprays have been performed for four different fuels, namely the diesel fuel DF2, n-heptane (HEP), the European-normed EN 590 fuel and the HVO fuel. The performance of these fuels have been compared with each 
other and with the appropriate experimental data used in Chap. 4. The evaluation criteria include the liquid and vapor penetrations, the drop size, flow velocity, flow vorticity, turbulent kinetic energy and the fuel vapor/air mixing.

\subsection{Introduction}

\subsubsection{ReFuel project}

Alternative fuels with high cetane numbers and lack of aromatic components can make a significant contribution towards the reduction of greenhouse gas emissions. The search for such alternative fuels is the main goal of many research projects, including the Finlandbased research project ReFuel. Hydrotreated vegetable oil (HVO), called NExBTL by the Neste Oil Company, is such a fuel and is the focus of the investigation in this study.

HVO is a renewable synthetic diesel hydrocarbon fuel with properties similar to gas-toliquid (GTL) diesel fuel. The absence of sulfur, aromatics and oxygenates greatly reduces the emissions of carbon monoxide $(\mathrm{CO})$, nitrogen oxides $\left(N \mathrm{O}_{x}\right)$, hydrocarbons $(H C)$ and particulate matter $(P M)$. The high cetane values between 84 and 99 make $\mathrm{HVO}$ an attractive alternative fuel for high-pressure on-road diesel engines. A low cloud point $\left(-30^{\circ} \mathrm{C}\right)$ allows its use in cold weather, which makes it an all-year-round fuel. In addition, its storage stability is as good as hydrocarbon fuels.

EN 590 describes the physical properties that an automotive diesel fuel must satisfy if it is to be sold in the European Union. In comparison with EN 590, HVO has a lower density 
and a higher cetane number. Therefore, the suitability of HVO as a single fuel in currently available fuel injection systems has to be evaluated. To aid in this evaluation, this chapter investigates various spray properties of HVO in controlled conditions and compares them with other conventional fuels including EN 590.

\subsubsection{Fuel spray properties}

The fuel properties that influence the spray performance include the density, vapor pressure, latent heat, specific heat, viscosity and surface tension. The symbols and units of these properties are listed in Table 5.1. All these properties are temperature dependent and are plotted in Fig. 5.1. The fuel properties of HVO and EN 590 were coded into the OpenFOAM fuel library as is documented in Ref. ${ }^{30}$ and Appendix A.3.

\section{Table 5.1}

Fuel properties related to the spray performance.

\begin{tabular}{ccc}
\hline Symbol & Name & Units \\
\hline$\rho$ & Density & {$\left[\mathrm{kg} / \mathrm{m}^{3}\right]$} \\
$p_{v}$ & Vapor pressure & {$[\mathrm{Pa}]$} \\
$h_{l}$ & Heat of vaporization & {$[\mathrm{J} / \mathrm{Kg}]$} \\
$c_{p}$ & Specific heat & {$[\mathrm{J} / \mathrm{Kg} \cdot \mathrm{K}]$} \\
$\mu$ & Dynamic Viscosity & {$[\mathrm{Pa} \cdot \mathrm{s}]$} \\
$\sigma$ & Surface tension & {$[\mathrm{N} / \mathrm{m}]$} \\
\hline
\end{tabular}

The liquid density data satisfy $\rho_{H E P}<\rho_{D F 2}<\rho_{H V O}<\rho_{E N 590}$. They are important in the prediction of the droplet momentum relaxation timescale, $\tau$, and the magnitude of the pressure dependent injection velocity, $U_{i n j}=\sqrt{2\left(p_{i n j}-p_{\text {air }}\right) / \rho_{l}}$. The heavier the fuel, the smaller the magnitude of the injection velocity. The values of the air pressure $p_{\text {air }}$ and the 
injection pressure $p_{i n j}$ can be found in Table 4.1 .

The relaxation time $\tau$ is the characteristic time for a droplet to decelerate from its injection velocity to the velocity of the surrounding gas, i.e., the time until the relative velocity of the droplet is zero. According to Eq. (4.11), the relaxation timescale $\tau$ depends on the magnitude of the relative velocity $\left\|\mathbf{u}_{r e l}\right\|$. Assuming that $\left\|\mathbf{u}_{r e l}\right\|=U_{\text {inj }}$, the corresponding momentum relaxation time $\tau$ is plotted for a drop with diameter $\mathrm{d}=0.2 \mathrm{~mm}$ and a fixed gas kinematic viscosity of $15.11 \mathrm{~m} 2 / \mathrm{s}$ for the non-evaporating Case 1 in Fig. 5.2. The values of $\tau$ for the four fuels are ordered as $\tau_{H V O}<\tau_{E N 590}<\tau_{D F 2}<\tau_{H E P}$ for low temperatures $(<400 \mathrm{~K})$.

The liquid viscosity, $\mu$, and surface tension, $\sigma$, influence the droplet breakup via the drop oscillation frequency in Eq. (4.7). The liquid viscosity is ordered as $\mathrm{HEP}<\mathrm{DF} 2<\mathrm{HVO}$ $<$ EN 590. The surface tension is inversely proportional to the Weber number, defined in Eq. (4.8). The unknown surface tensions of HVO is assumed to be the same as that of EN 590, because HVO is similar to EN $590 .^{76}$

The vapor pressure, $p_{v}$, of $\mathrm{HVO}$ at $310.8 \mathrm{~K}$ is $3.8 \mathrm{kPa}$. The vapor pressure of $\mathrm{HVO}$ at other temperatures are estimated by shifting the EN 590 vapor pressure curve to the known pressure of $3.8 \mathrm{kPa}$ at the temperature $310.8 \mathrm{~K}$. N-heptane, the evaporating experimental fuel, exhibits a greater vapor pressure than the EN 590 and HVO. Generally, the greater the vapor pressure, the easier it is for the drop to evaporate. 

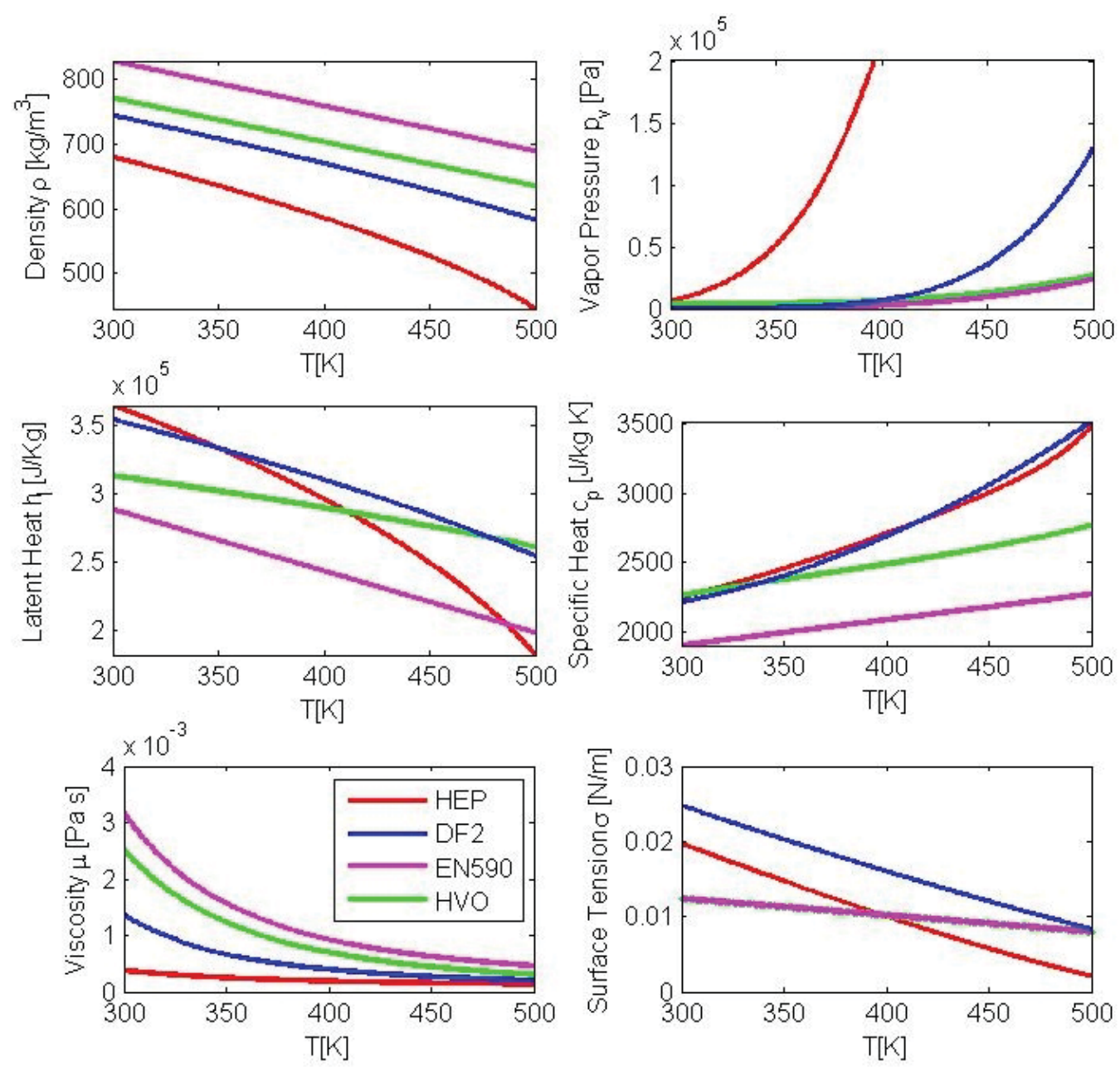

Figure 5.1: Fuel properties comparison.

The liquid latent heat, $h_{l}$, and specific heat, $c_{p}$, are two important fuel properties for evaporating sprays. Here, the specific heat of HVO is estimated by the weighted average of four components: $18.1 \%$ Pentadecane $\mathrm{C}_{15} \mathrm{H}_{32}, 31.1 \%$ Hexadecane $\mathrm{C}_{16} \mathrm{H}_{34}, 25.5 \%$ Heptadecane $\left(\mathrm{C}_{17} \mathrm{H}_{36}\right)$ and $25.3 \%$ Octadecane $\mathrm{C}_{18} \mathrm{H}_{38}{ }^{79}$ Compared to the other three fuels, the latent heat and specific heat of EN 590 is the smallest.

It is interesting to note that in Fig. 5.3, the thermal diffusivity, $\alpha$, is ordered as HEP $<$ DF2 $<$ EN $590<$ HVO. The thermal diffusivity is related to the heat conductivity $K$ by $\alpha \equiv \frac{K}{\rho_{l} c_{p}}$. 


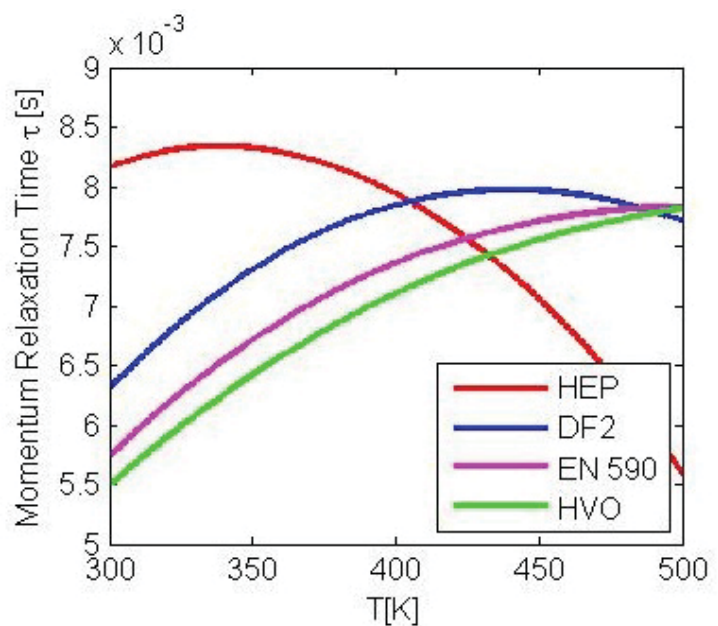

Figure 5.2: Momentum relaxation time $\tau$ of the non-evaporating Case 1 with $\mu_{g}=15.11 \mathrm{~m}^{2} / \mathrm{s}$ for a drop with diameter $d_{0}=0.2 \mathrm{~mm}$ and the injection velocity.

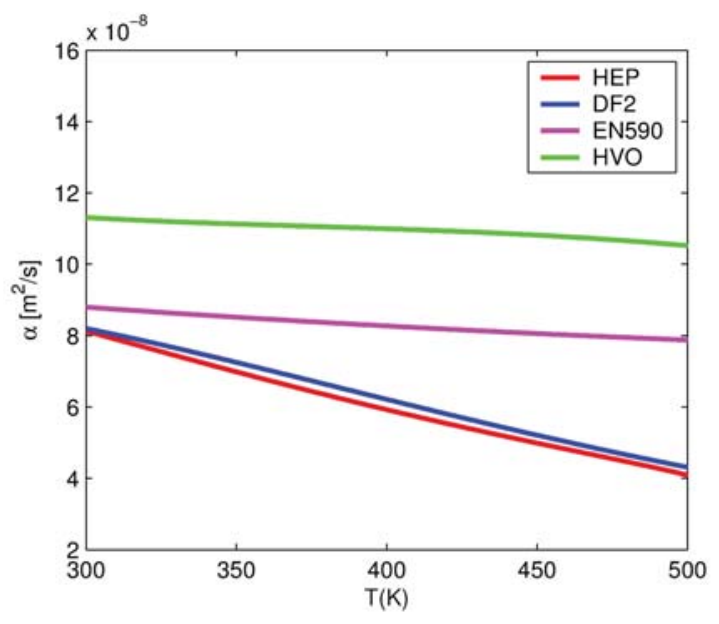

Figure 5.3: Thermal diffusivity $\alpha=\frac{K}{\rho c_{p}}$ and $\mathrm{K}$ is the thermal conductivity.

The fuel HVO has the highest thermal diffusivity. This means HVO fuel drops will rapidly increase their temperature to the surrounding gas temperature in the evaporating case.

Any temperature dependent property of the fuel is interpolated with an empirical function of temperature. The function expression and the interpolation coefficients used in this thesis are stored in the user-defined fuel libraries of OpenFOAM, as is documented in Appendix A.3. 


\subsection{Computation details}

The spray simulations reflect the experiments performed in a constant-volume combustion cell, as described in Chap. 4. In these experiments, liquid fuel was injected into a gas at rest under various conditions close to the ones encountered in diesel engines. Simulations have been performed for non-evaporating and evaporating sprays for the four fuels investigated in this chapter. These fuels are the idealized diesel fuel DF2 $\left(\mathrm{C}_{12} \mathrm{H}_{26}\right)$, n-heptane HEP $\left(C_{7} H_{16}\right)$, the normed diesel fuel EN 590 and the hydrotreated vegetable oil HVO. Note that the laboratory experiments of HVO and EN 590 have not been performed yet, hence the experimental data are not available. Thus in this chapter, we only compare the simulation results of EN 590 and HVO to the available experimental data of DF2 and HEP.

The geometry of the experiments is the same as the cylinder in Chapter 4. The various conditions of the spray experiments are also summarized in Table 4.1. The computational meshes are structured hexahedral, polar meshes which are the standard meshes in the mesh dependence studies in Chapter 4. For RANS simulation, there are 28x32x36 cells in radial, azimuthal and axial direction, and the smallest cell is located at the top center and has a height of $1 \mathrm{~mm}$ and a radius of 1mm. For LES simulation, there are 76x76x140 cells in radial, azimuthal and axial direction, and the smallest cell is located at the top center and has a height of $0.5 \mathrm{~mm}$ and a radius of $0.5 \mathrm{~mm}$. 


\subsection{LES/RANS results and discussions}

\subsubsection{Non-evaporating sprays}

In the non-evaporating cases 1-3, the different fuels are injected into the cylinder with gas pressures at 1.1 MPa, $3 \mathrm{MPa}$ and $5 \mathrm{MPa}$. The spray tip penetrations of the fuel DF2 serve

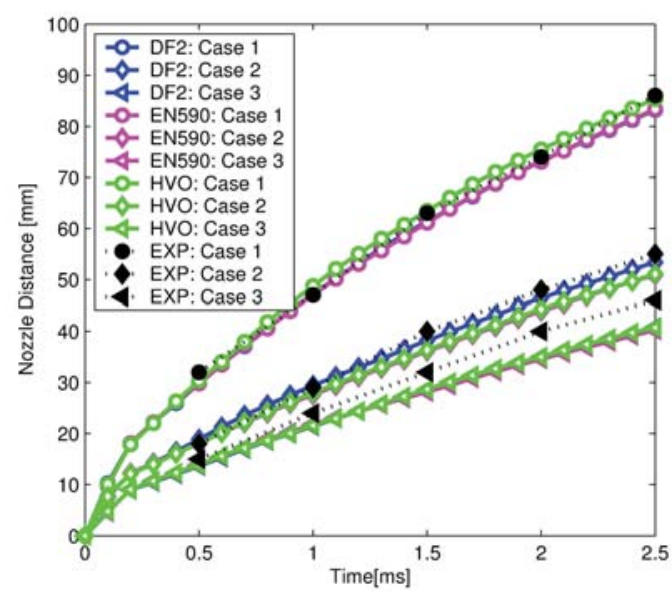

(a) RANS

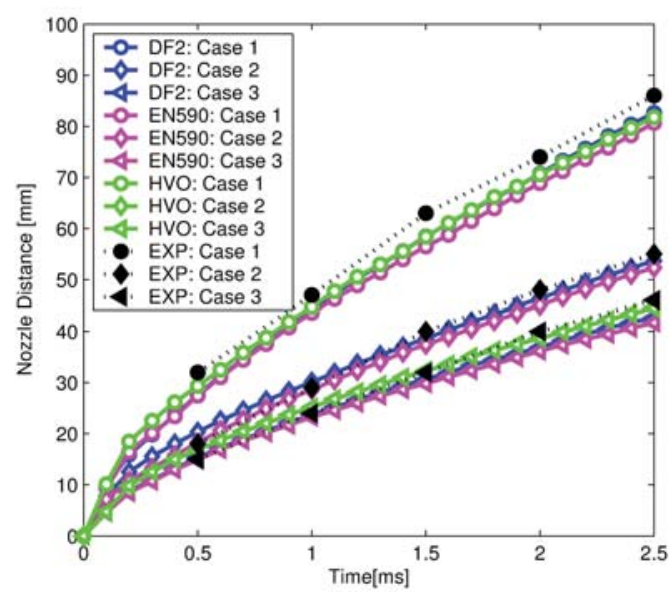

(b) LES

Figure 5.4: Temporal change in spray tip penetration of non-evaporating sprays with different fuels for the RANS and LES simulations.

as a reference and are validated with the experimental data, as is shown in Figs. 5.4(a) and 5.4(b) for the RANS and the LES, respectively. Both for the RANS and LES simulations, the spray penetrations of HVO and EN 590 are seen to be very close to the ones of DF2. The similarities of the liquid penetration curves suggest that the spray models are sufficiently universal to make correct predictions for the alternative fuel HVO without any special adjustments. Additionally, it can also be concluded that HVO can be used as an alternative fuel in current diesel engines without changes to the geometry of the combustion chamber. 

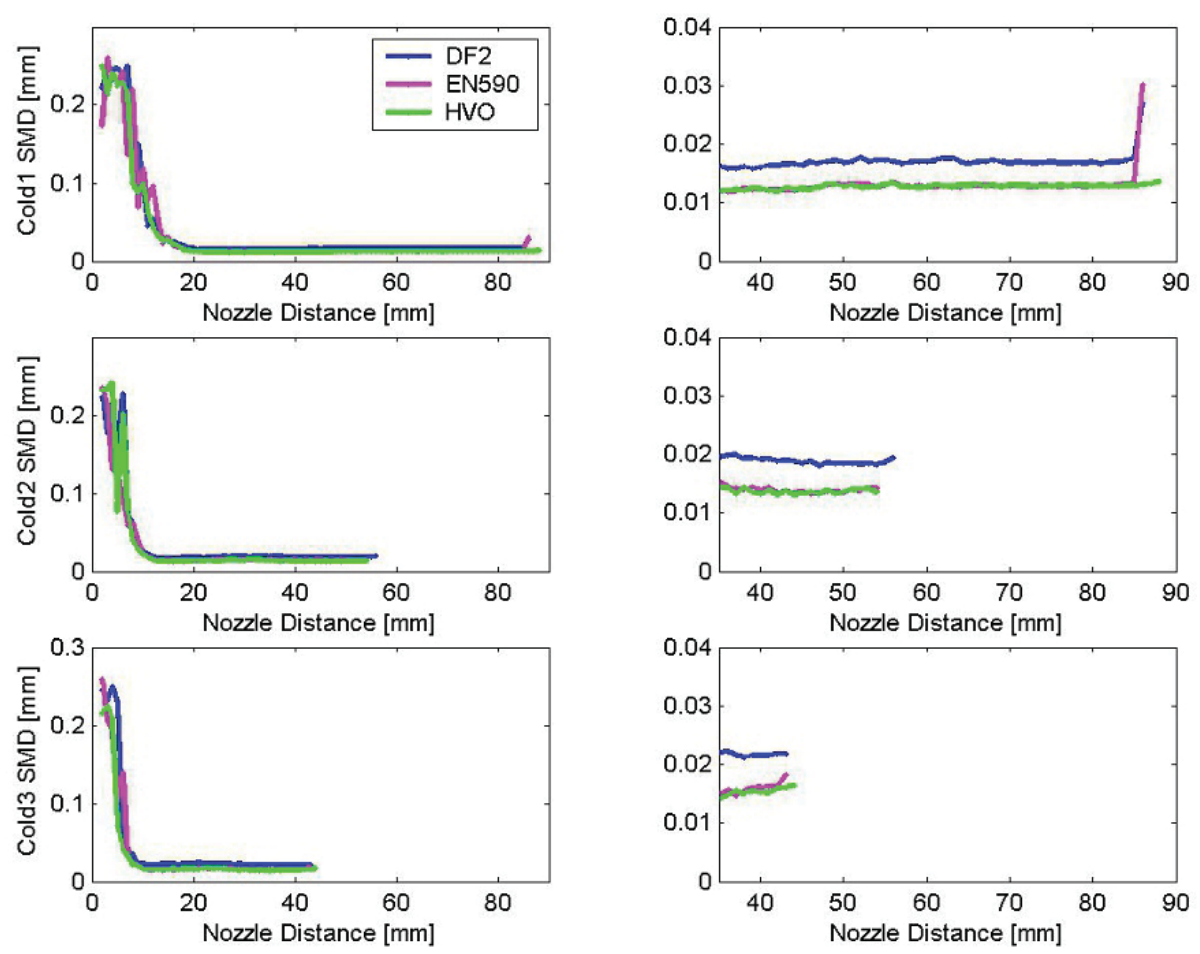

Figure 5.5: Cross-sectional SMD of the non-evaporating cases at the injection time $=0.0024 \mathrm{~s}$ for the RANS simulations.
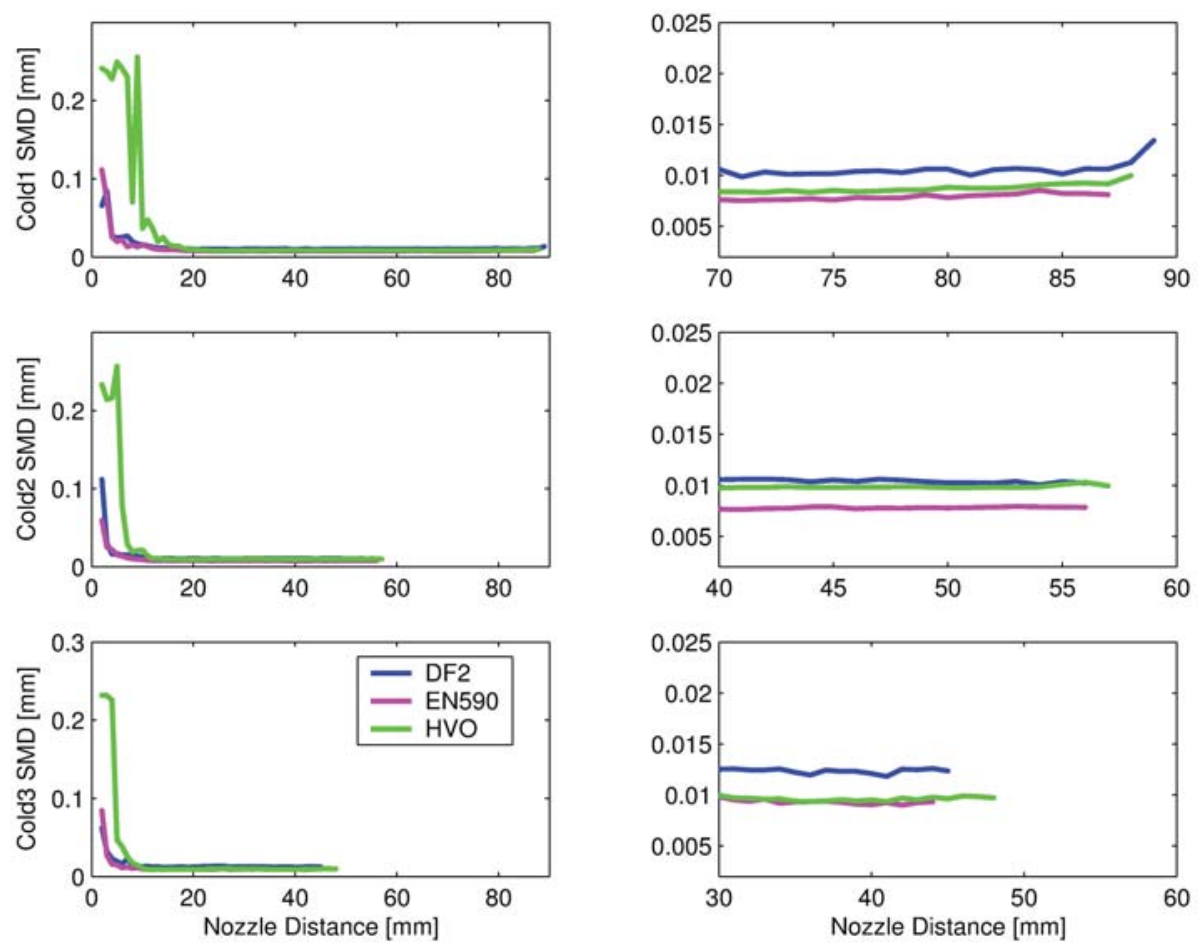

Figure 5.6: Cross-sectional SMD of the non-evaporating cases at the injection time $=0.0024 \mathrm{~s}$ for the LES simulations. 
Fig. 5.5 and Fig. 5.6 show the cross-sectional Sauter Mean Diameter (SMD) of the three fuels, DF2, EN 590 and HVO, for the RANS and the LES. The plots in the right-hand column show the details of the curves near their minimal values. As is seen, both in the RANS and the LES, DF2 has the largest SMD near the spray tip in all cases. In particular, stable droplets, that is droplets below the critical Weber number, have large radii.

In the RANS simulations, the other two fuels, HVO and EN 590, show almost identical behavior. However, in the LES simulations, the behavior of EN 590 and HVO are different. First, the downstream SMDs of HVO are larger than EN 590, which is not observed in the RANS. Further investigation is necessary, especially considered that EN 590 and HVO have the identical surface tension. (Recall that the surface tension as a function of temperature is taken to be the same for the two fuels.) Second, the SMDs of HVO near the nozzle is much larger than the other two fuels. This is also observed in Fig. 5.7(f).As discussed above, HVO has the smallest momentum relaxation time, $\tau$, as shown in Fig. 5.2, and thus a greater drop deceleration by Eq. (4.10). Then the magnitude of the relative velocity of HVO is almost the smallest, which leads the smallest Weber number with the same drop diameter $d_{0}$, i.e. the HVO drops are harder to breakup at the very beginning of the injection. Considered that HVO and EN 590 have the same surface tension, the main cause of the larger drop size is the smaller relative velocity predicted by HVO.

HVO has the largest droplets at the nozzle exit. This means that the liquid to air surface ratio for HVO is the smallest. Therefore, the momentum exchange between the liquid and the 


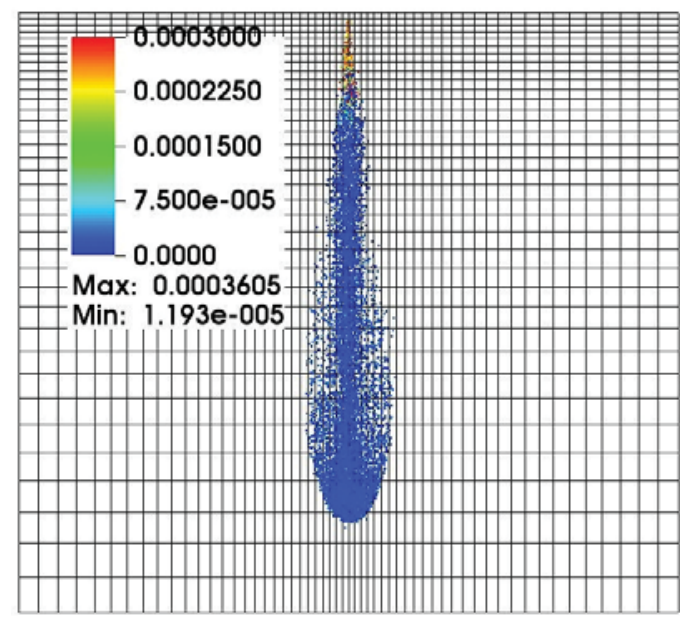

(a) RANS: DF2

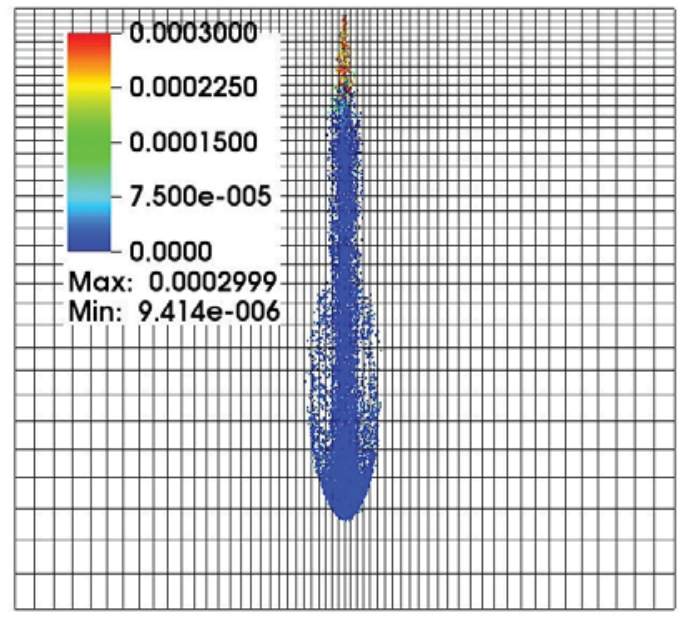

(c) RANS: EN 590

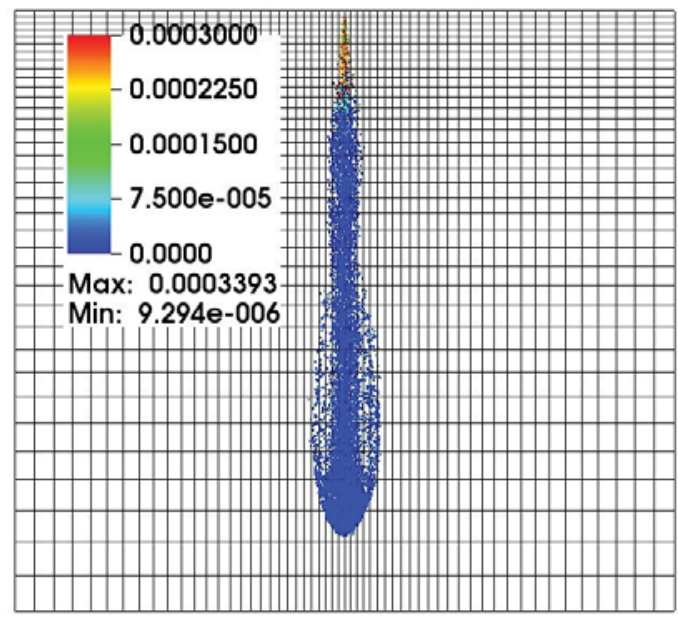

(e) RANS: HVO

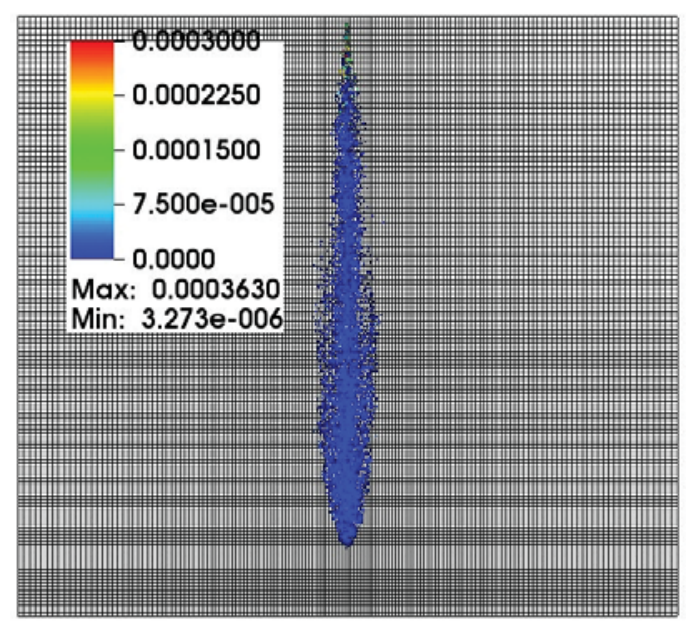

(b) LES: DF2

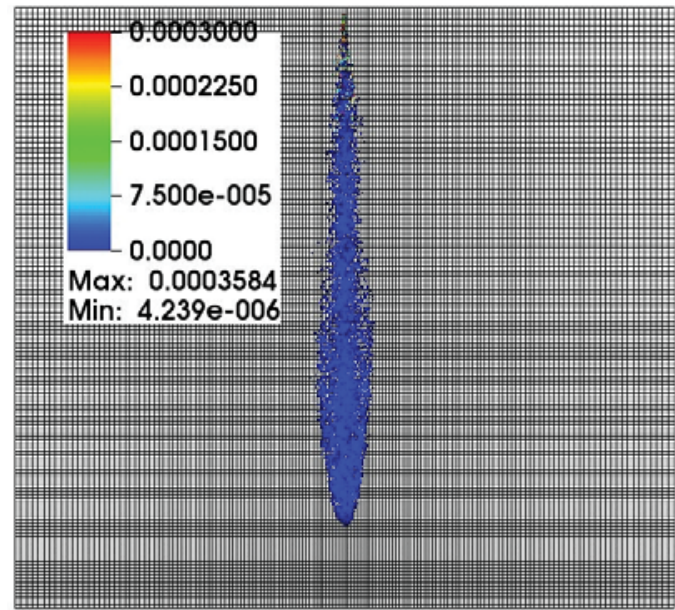

(d) LES: EN 590

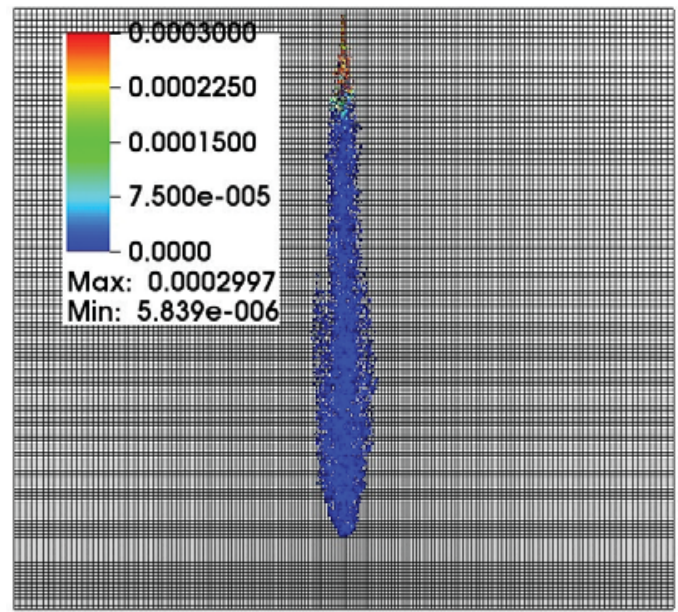

(f) LES: HVO

Figure 5.7: Spray droplet diameter in $m$ of the non-evaporating Case 1 using different fuels at time $=0.0024 \mathrm{~s}$ for the RANS and LES simulations. 


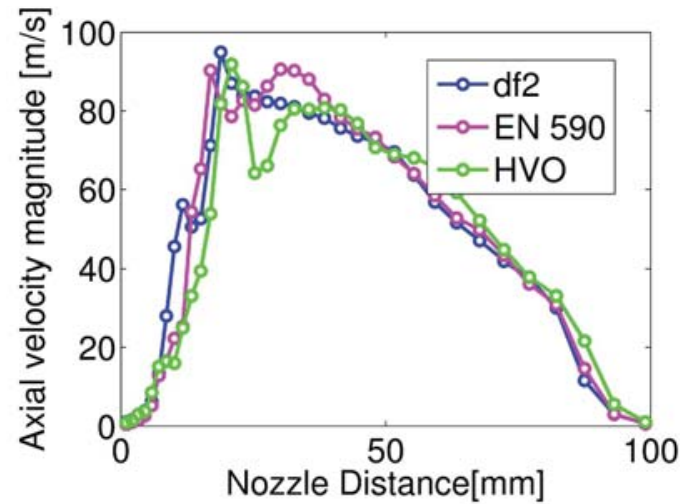

(a) RANS

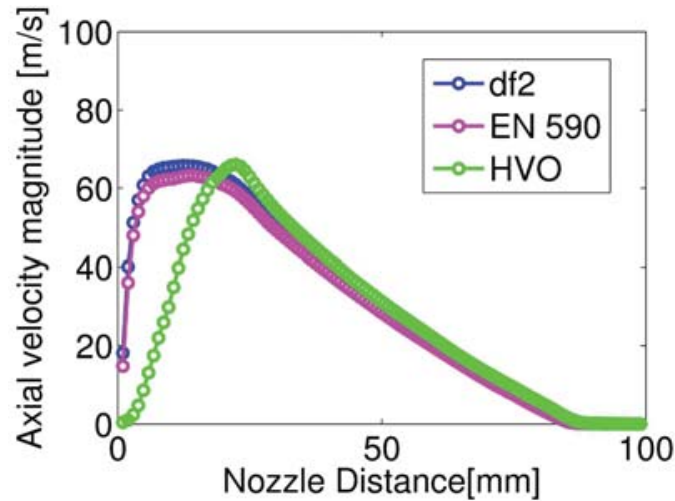

(b) LES

Figure 5.8: Magnitude of axial velocity versus nozzle distance along the centerline of the non-evaporating Case 1 at time $=0.0024 \mathrm{~s}$.

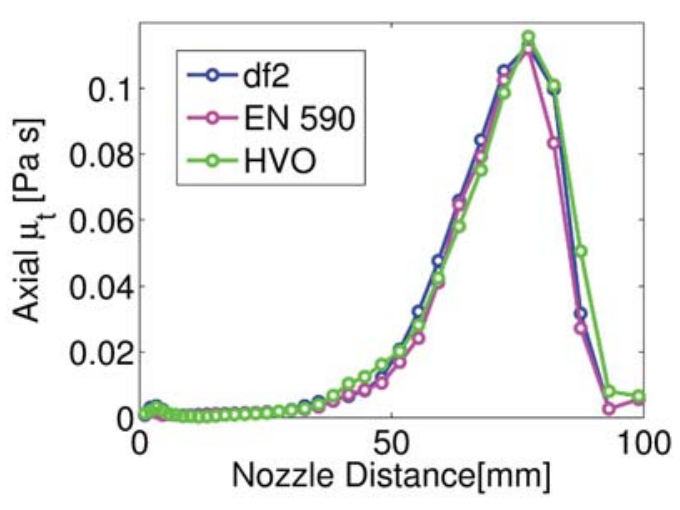

(a) RANS

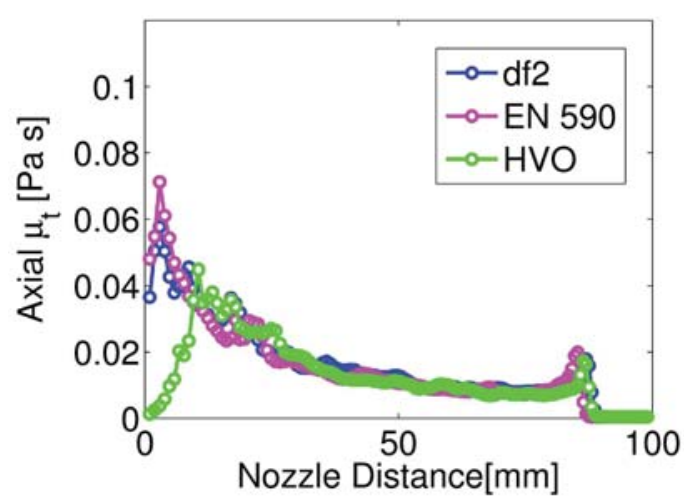

(b) LES

Figure 5.9: Turbulent viscosity $\mu_{t}$ versus nozzle distance along the centerline of the non-evaporating Case 1 at time $=0.0024 \mathrm{~s}$.

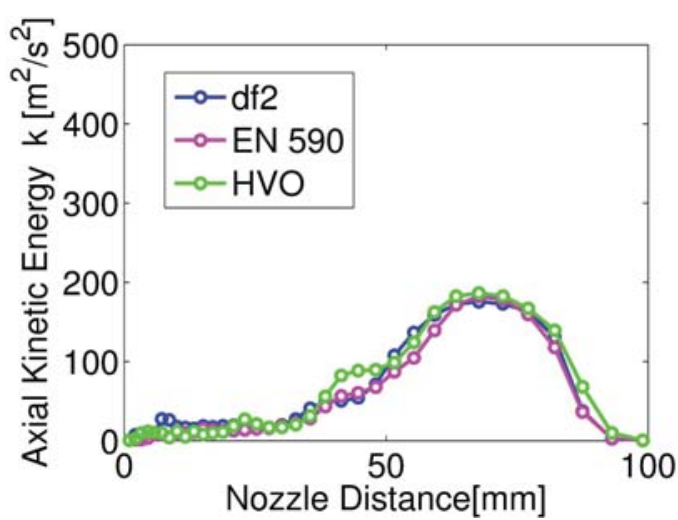

(a) RANS

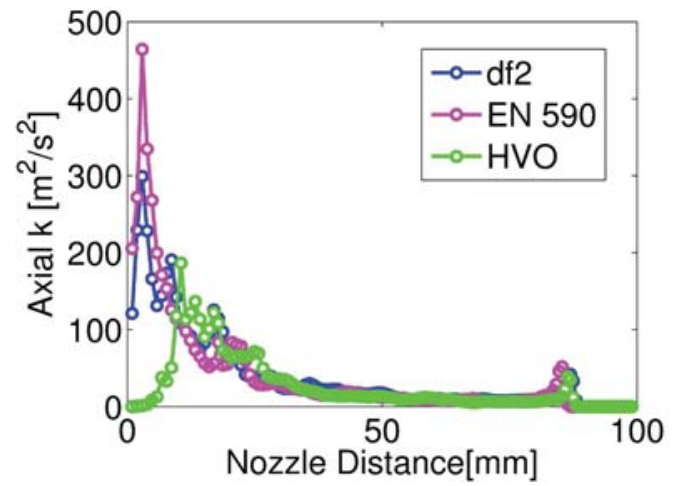

(b) LES

Figure 5.10: Turbulent kinetic energy $k$ versus nozzle distance along the centerline of the non-evaporating Case 1 at time $=0.0024 \mathrm{~s}$. 


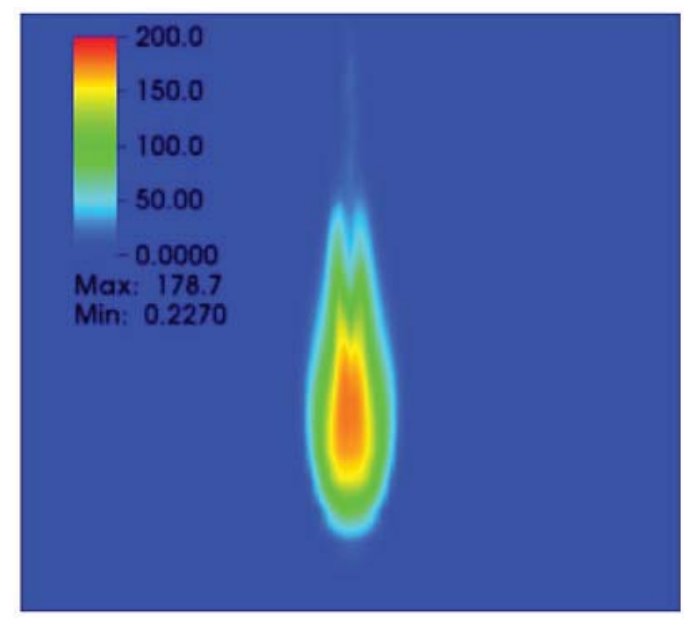

(a) RANS: DF2

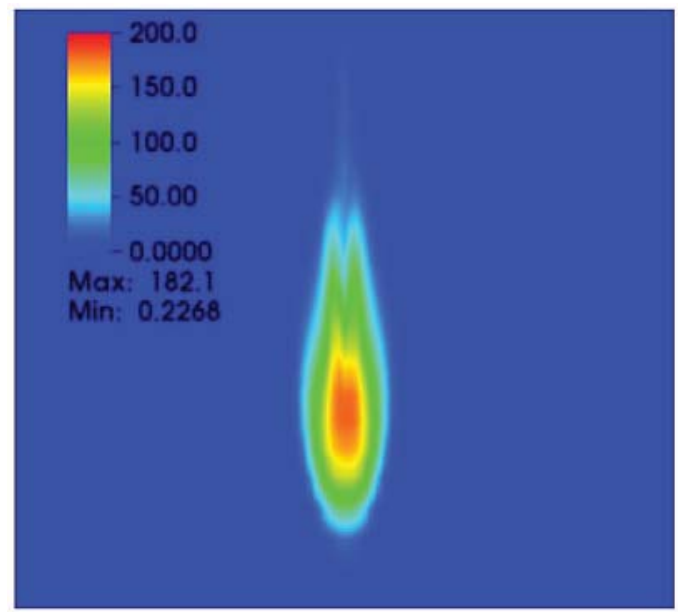

(c) RANS: EN 590

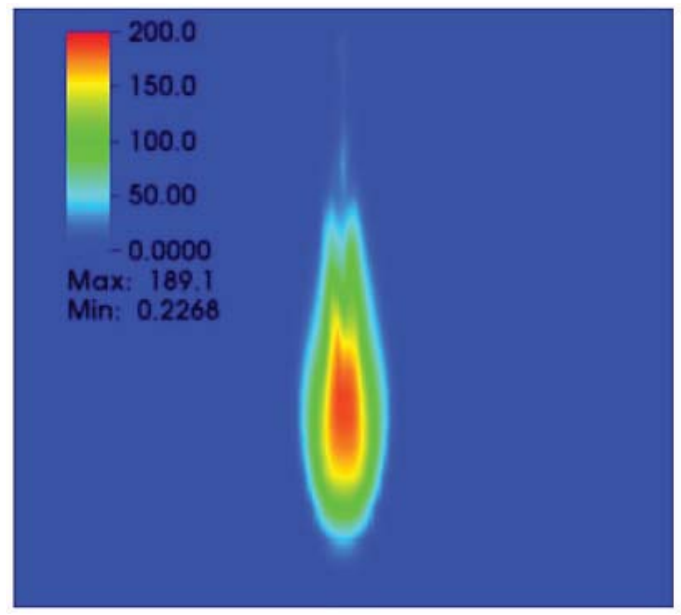

(e) RANS: HVO

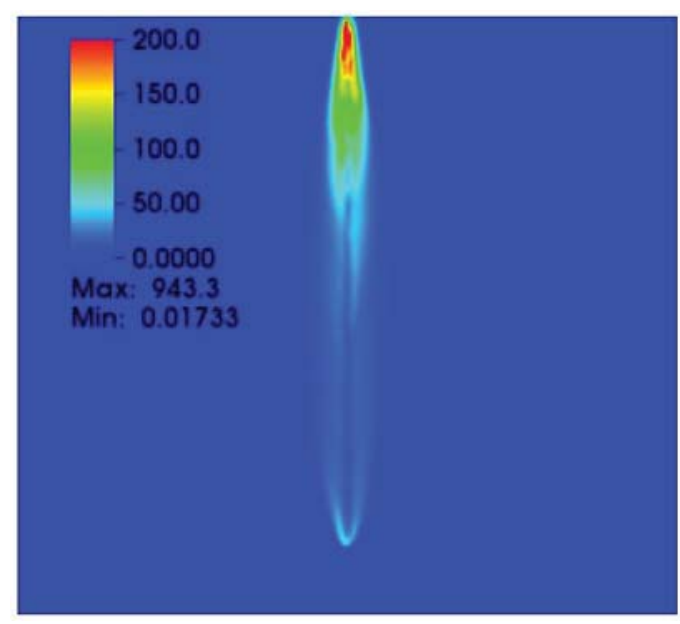

(b) LES: DF2

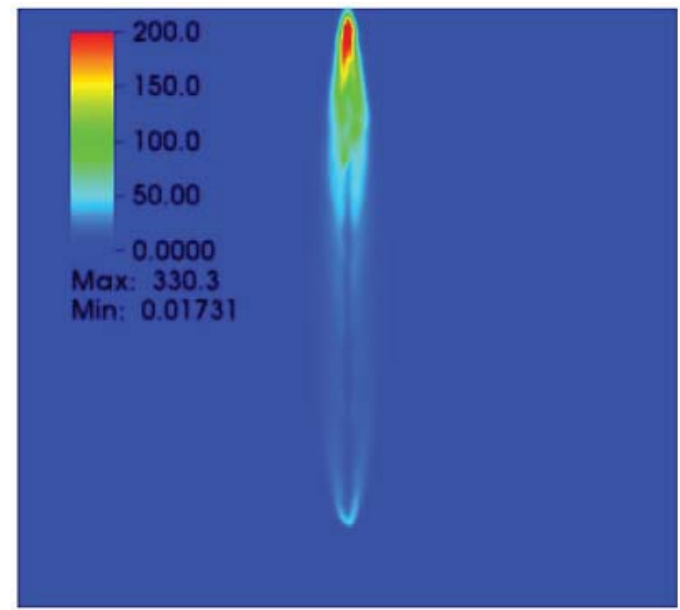

(d) LES: EN 590

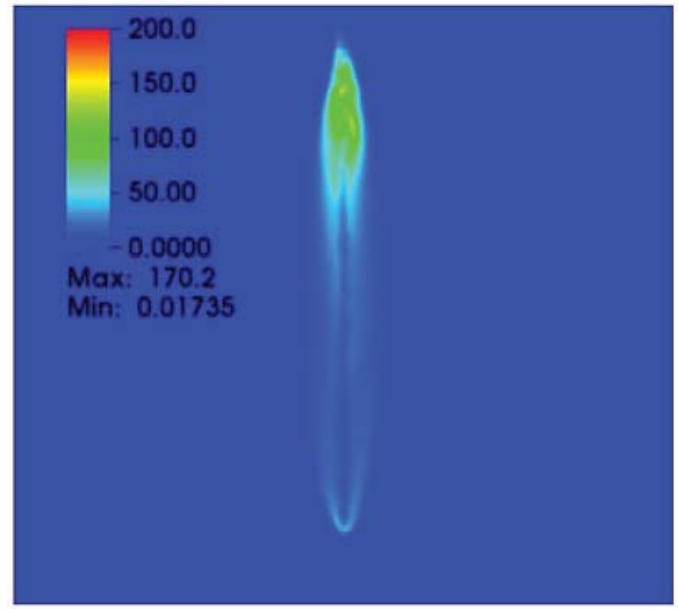

(f) LES: HVO

Figure 5.11: Turbulent kinetic energy in $\mathrm{m}^{2} / \mathrm{s}^{2}$ of the non-evaporating Case 1 using different fuels at time $=0.0024 \mathrm{~s}$ for the RANS and LES simulations. 
gas is also the smallest. This results in the smallest gas velocity as is illustrated in Fig. 5.8. As a consequence, the flow-induced turbulence is the smallest, which is also reflected by the turbulent viscosity in Fig. 5.9(b) and the turbulence kinetic energy in Fig. 5.10(b). More details of the turbulent kinetic energy are shown in the cross sectional plots including for the RANS and the LES in Fig. 5.11. In RANS, the turbulent kinetic energy of the three fuels are very similar. In LES, HVO has the smallest SGS turbulent kinetic energy near the nozzle.

The magnitude of the gas phase vorticity, $\|\omega\|=\|\nabla \times \mathbf{u}\|$, is shown in Fig. 5.12 for the RANS and the LES. The vorticity is generated by the interaction between droplets and the undisturbed gas. As in Chap. 4, the vorticity in the LES simulations is spread much more evenly throughout the spray. Obviously, the RANS simulations failed to capture the droplet circulation near the spray tip and the nozzle exit. In the LES simulations, the HVO case has an much lower vorticity near the nozzle. This coincides with the fuel drop SMDs near the nozzle, i.e., the large SMDs correspond to the small vorticities.

\subsubsection{Evaporating spray}

The vapor penetrations (dashed lines) and liquid penetrations (solid lines) are shown in Fig. 5.13(a) for the RANS and in Fig. 5.13(b) for the LES; the comparisons with the experiments apply to the n-heptane simulations. The liquid penetrations of HVO and EN 590 are

considerably larger than the ones for n-heptane. This, as discussed below in more detail, is 


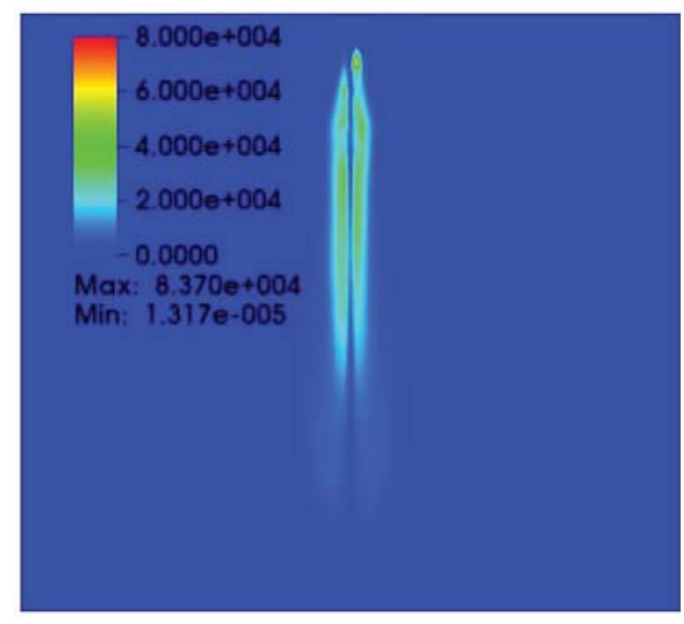

(a) RANS: DF2

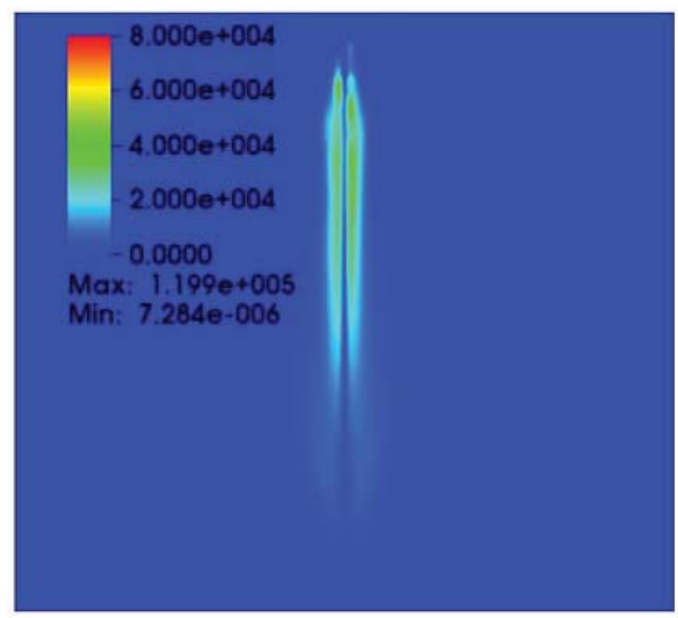

(c) RANS: EN 590

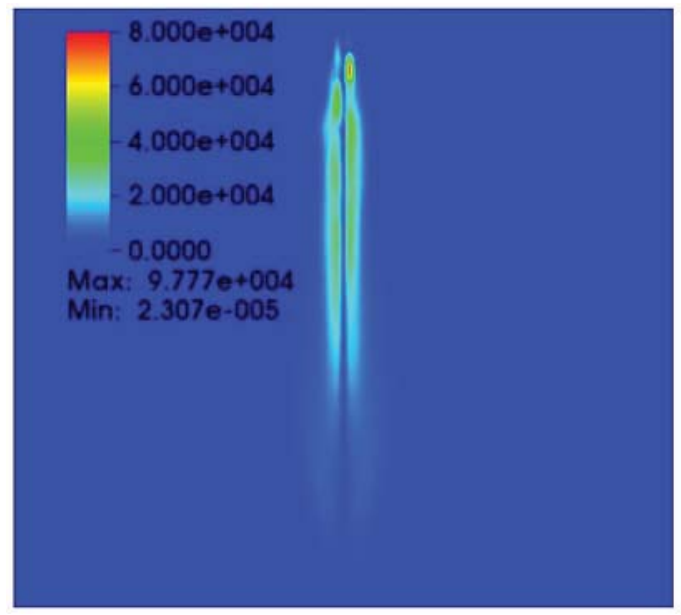

(e) RANS: HVO

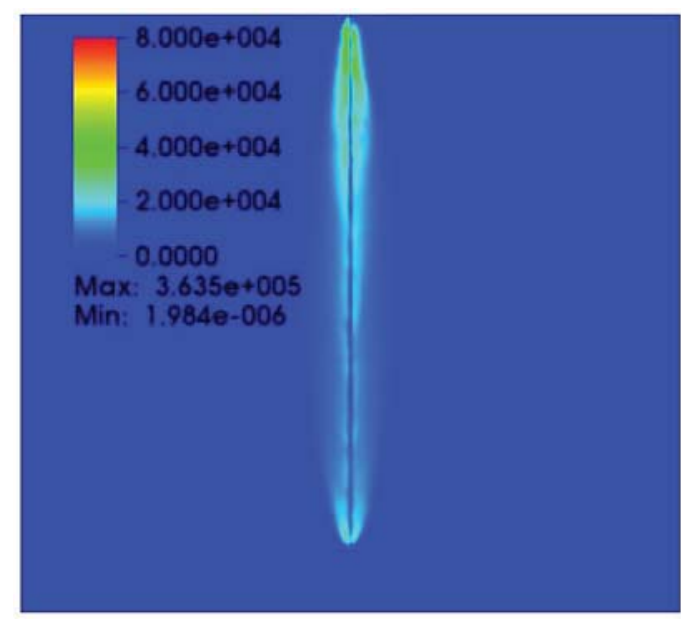

(b) LES: DF2

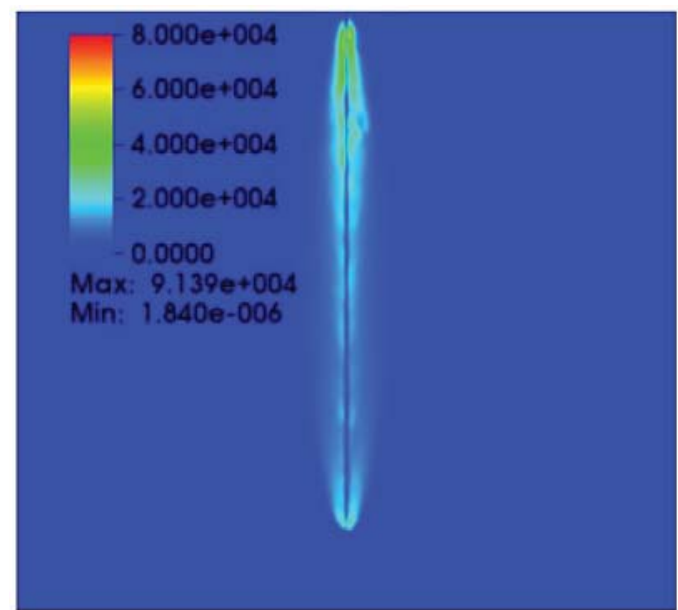

(d) LES: EN 590

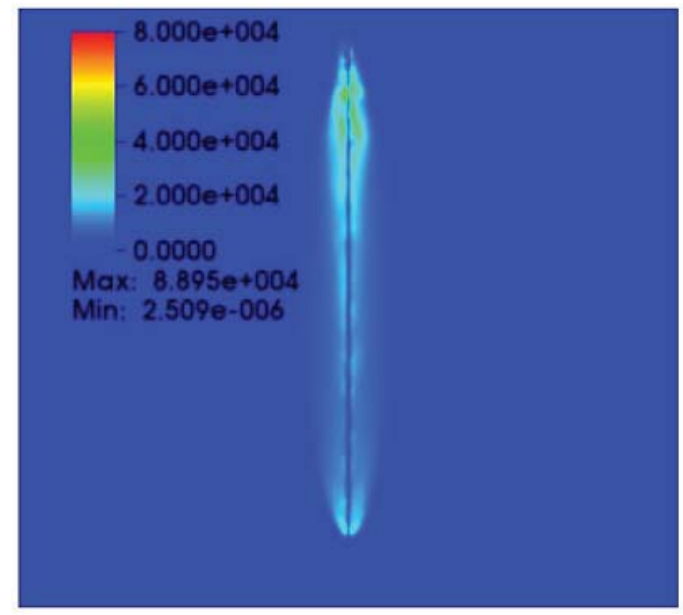

(f) LES: HVO

Figure 5.12: Magnitude of vorticity in $s^{-1}$ of the non-evaporating Case 1 using different fuels at time $=0.0024 \mathrm{~s}$ for the RANS and LES simulations. 


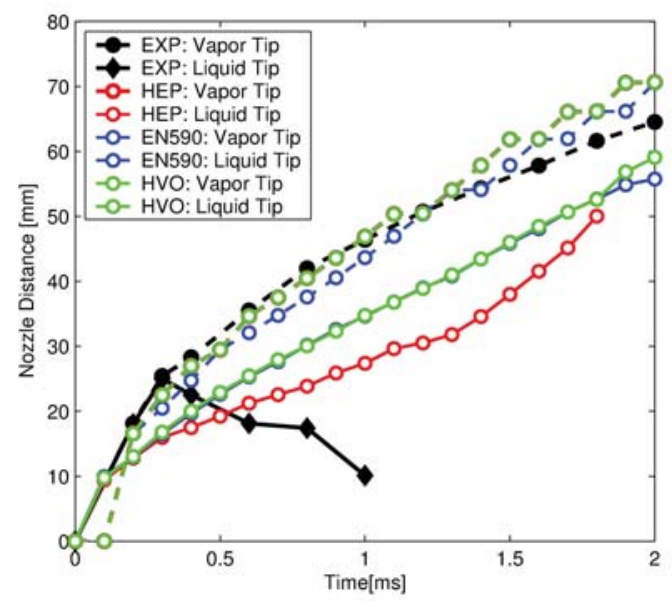

(a) RANS

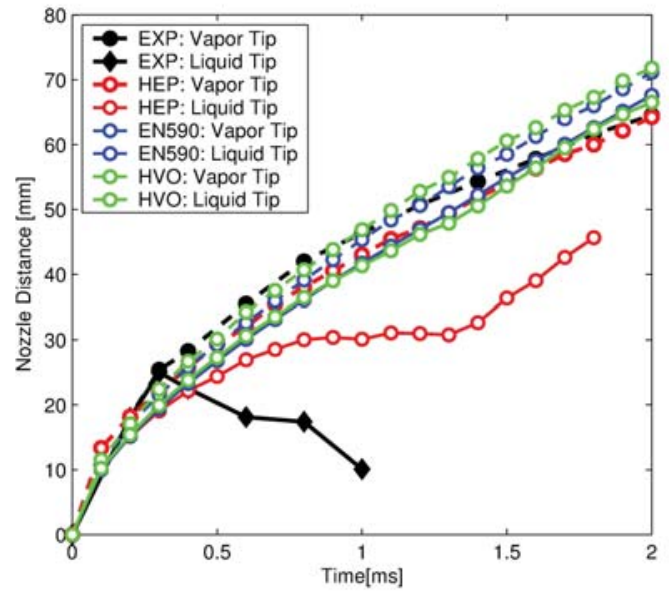

(b) LES

Figure 5.13: Temporal change in spray tip penetration of evaporating sprays with different fuel for the RANS and LES simulations.

consistent with the fact that HVO and EN 590 have smaller evaporation rates, a fact that is consistent with the higher vapor pressure of n-heptane shown in Fig. 5.1.

The drop temperatures of the three fuel sprays are shown in Figs. 5.14 and 5.15 on a cut plane through the spray at the injection times of $0.8 \mathrm{~ms}$ and $1.6 \mathrm{~ms}$ for the RANS and the LES respectively. These plots show that the number of droplets for the n-heptane is considerably reduced for the larger injection time, i.e., more droplets have evaporated. This is a clear indication that the evaporation rate of n-heptane is larger than that of HVO and EN 590. Another indication is that the maximum temperature $\left(T_{\max } \approx 540 \mathrm{~K}\right)$ of the n-heptane droplets is lower than the maximum temperature $\left(T_{\max } \approx 658 \mathrm{~K}\right)$ of the EN 590 and HVO droplets. The droplets with the temperature greater than $T_{\max }$ are evaporated and turned from the liquid states into the gas states. As mentioned above, the larger evaporation rate of n-heptane is the cause for the smaller liquid penetration shown in Fig. 5.13. 


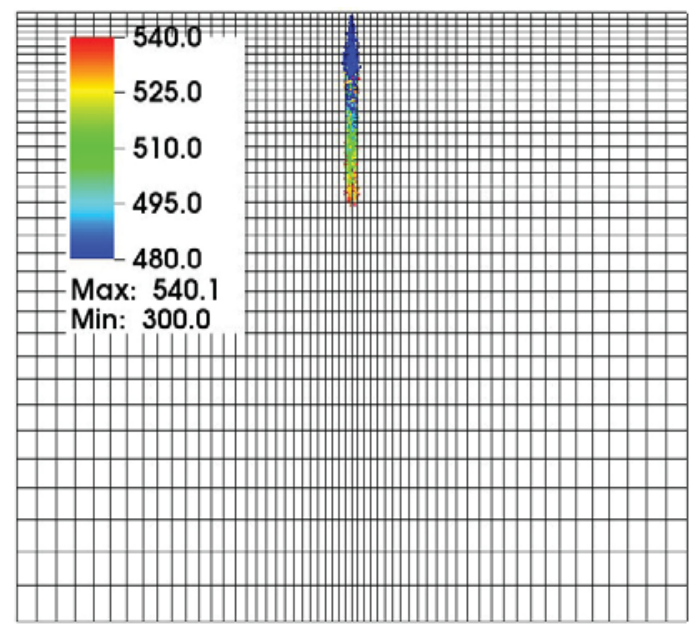

(a) RANS: HEP

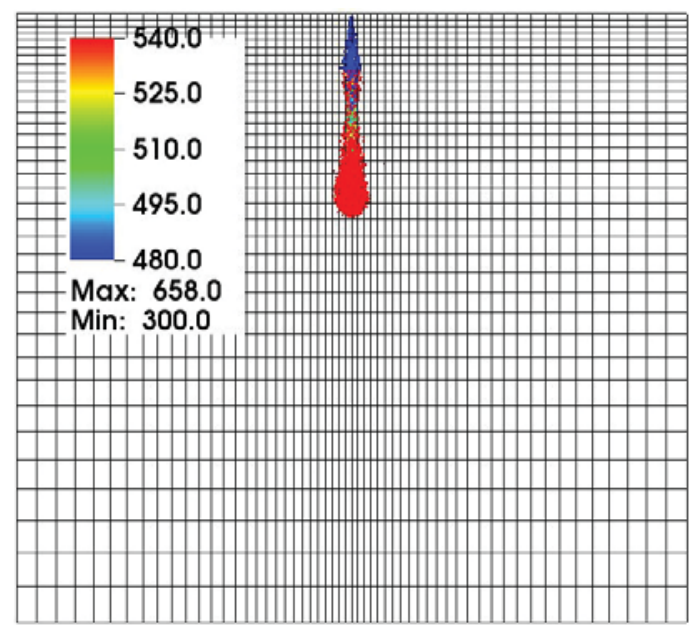

(c) RANS: EN 590

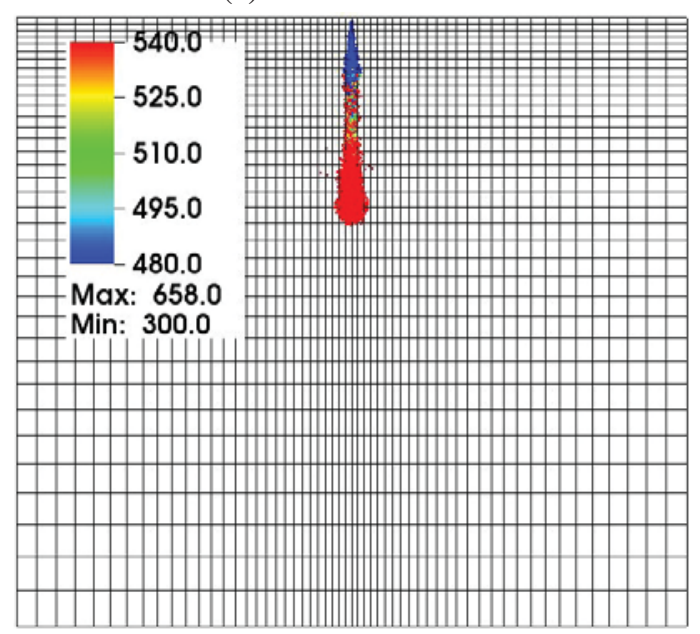

(e) RANS: HVO

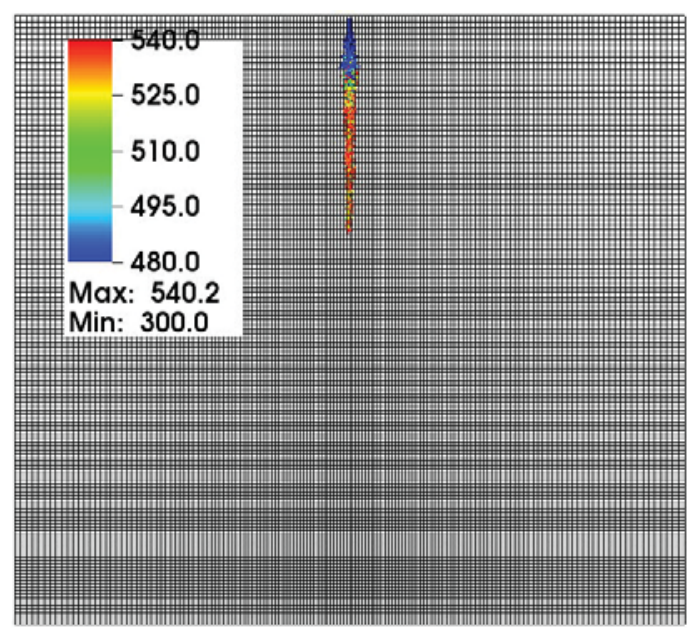

(b) LES: HEP

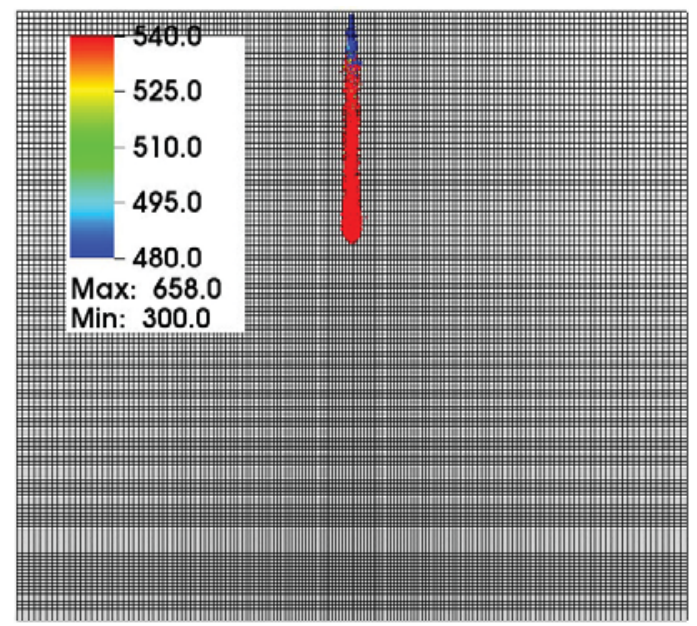

(d) LES: EN 590

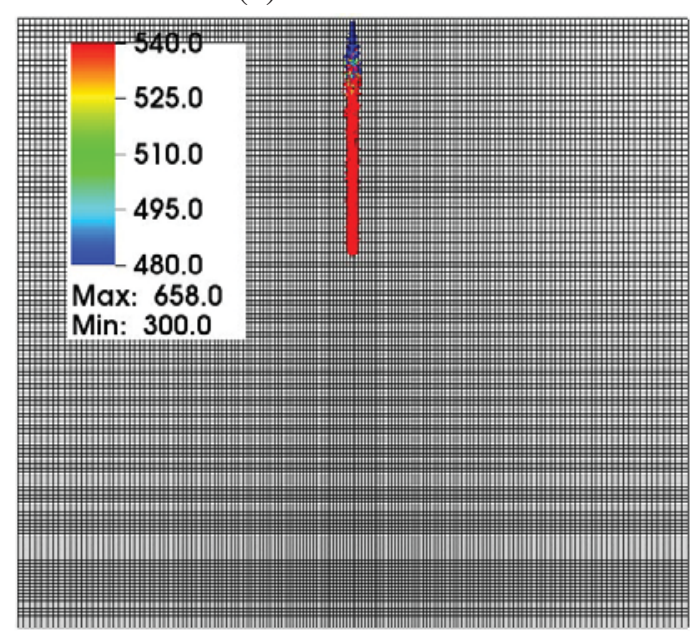

(f) LES: HVO

Figure 5.14: Spray droplet temperature in $K$ of the evaporating case using different fuels at time $=0.0008 \mathrm{~s}$ for the RANS and the LES simulations. 


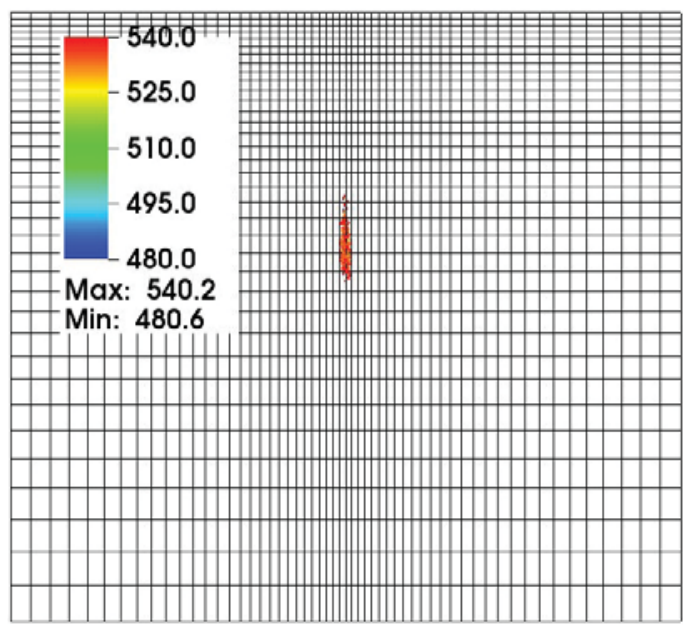

(a) RANS: HEP

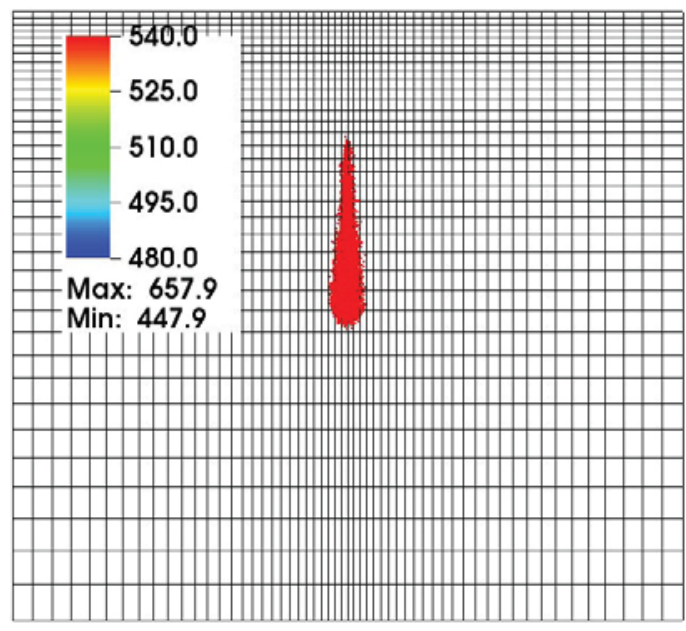

(c) RANS: EN 590

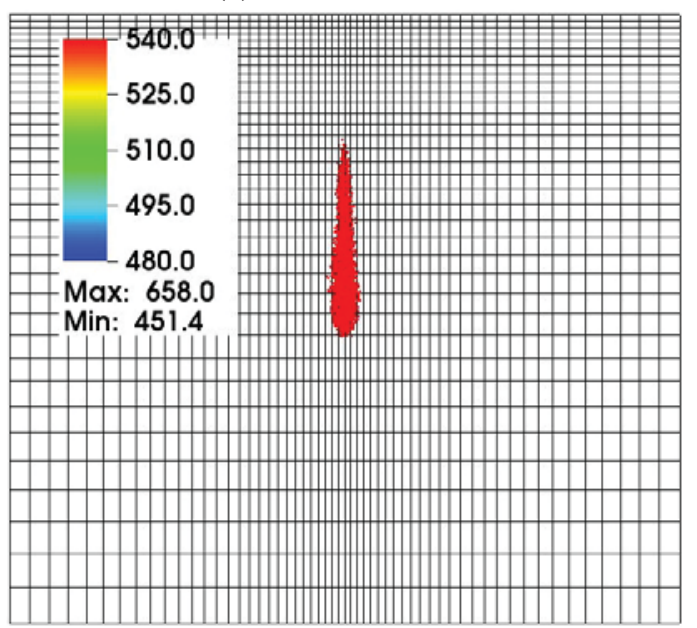

(e) RANS: HVO

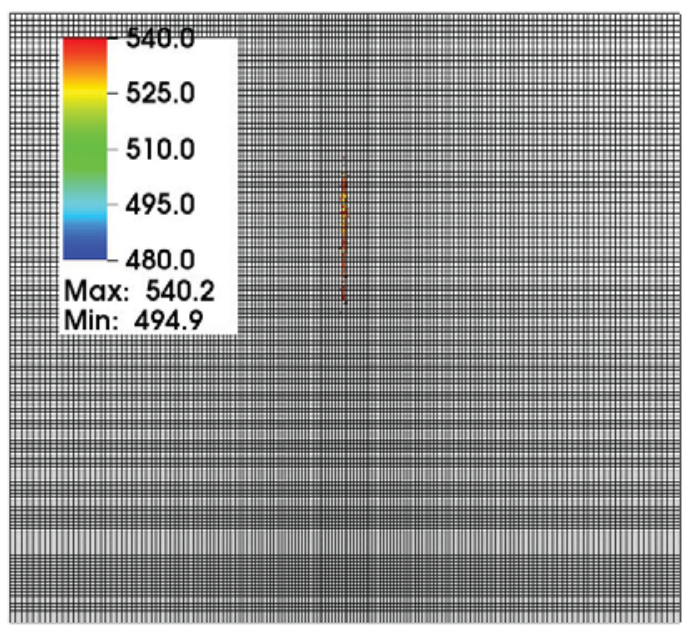

(b) LES: HEP

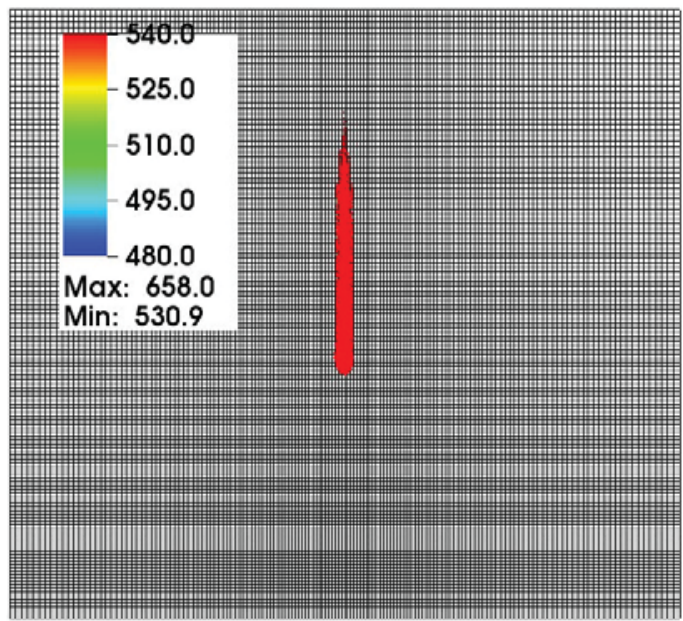

(d) LES: EN 590

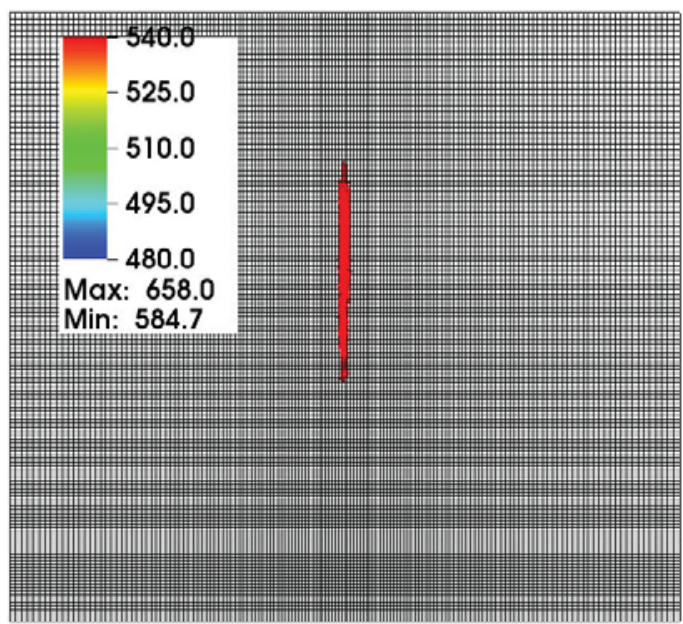

(f) LES: HVO

Figure 5.15: Spray droplet diameter in $\mathrm{K}$ of the evaporating case using different fuels at time $=0.0016 \mathrm{~s}$ for the RANS and the LES simulations. 


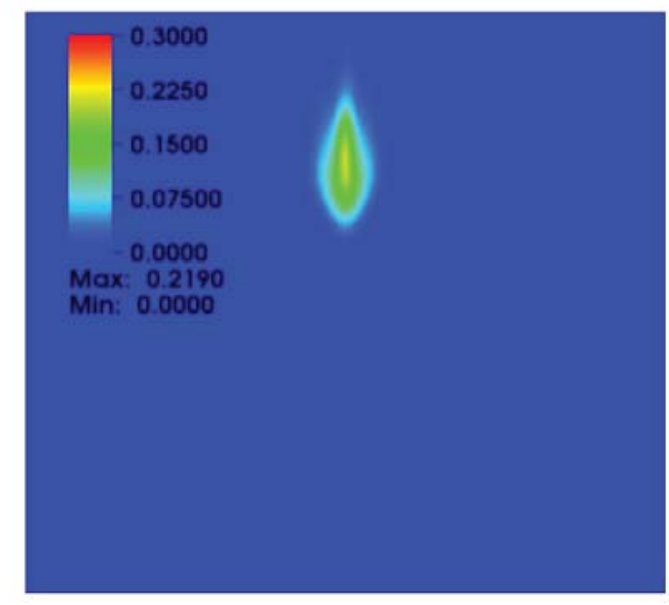

(a) RANS: HEP

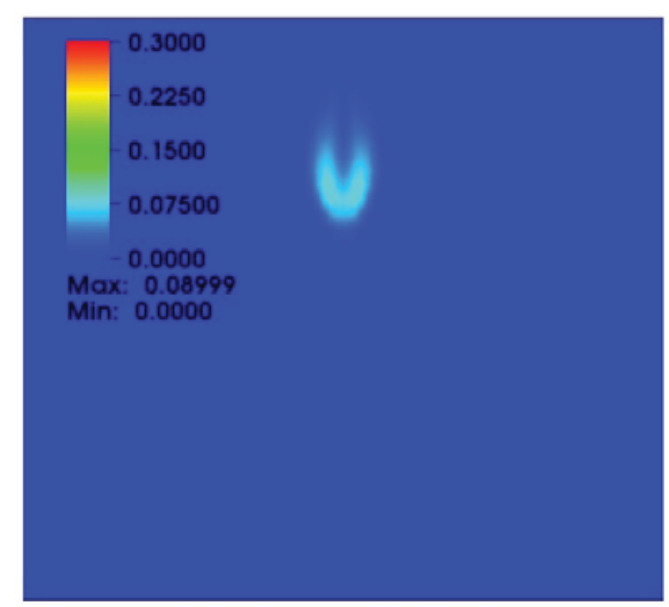

(c) RANS: EN 590

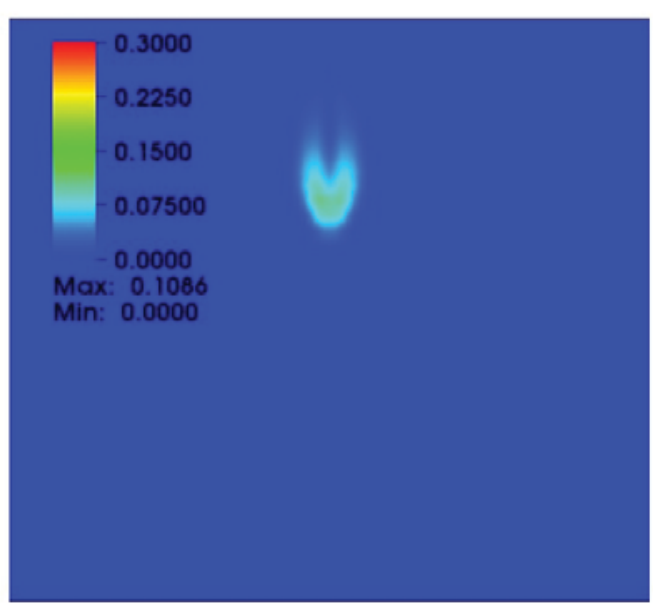

(e) RANS: HVO

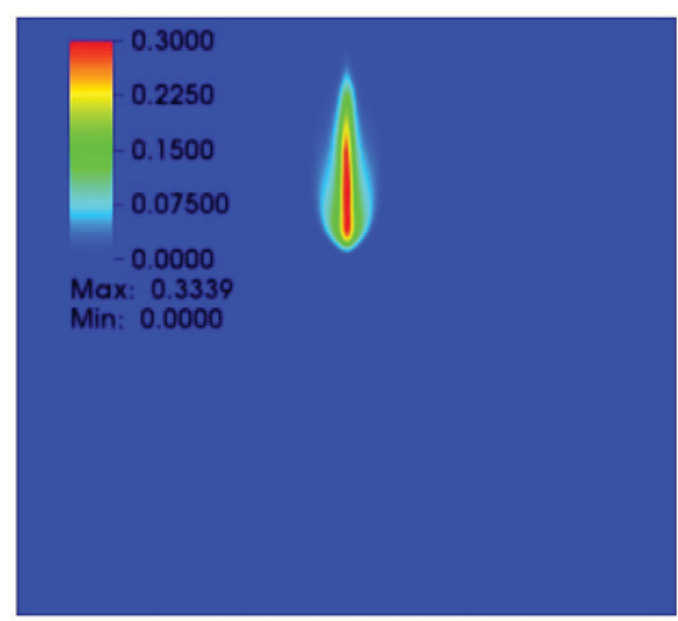

(b) LES: HEP

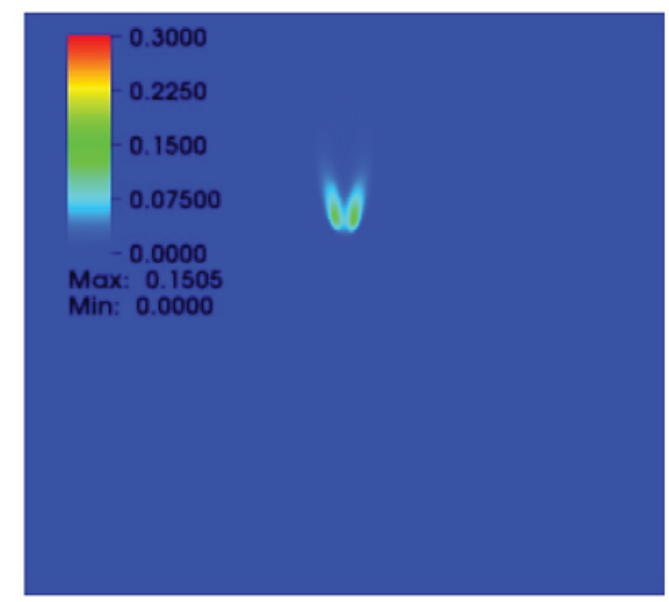

(d) LES: EN 590

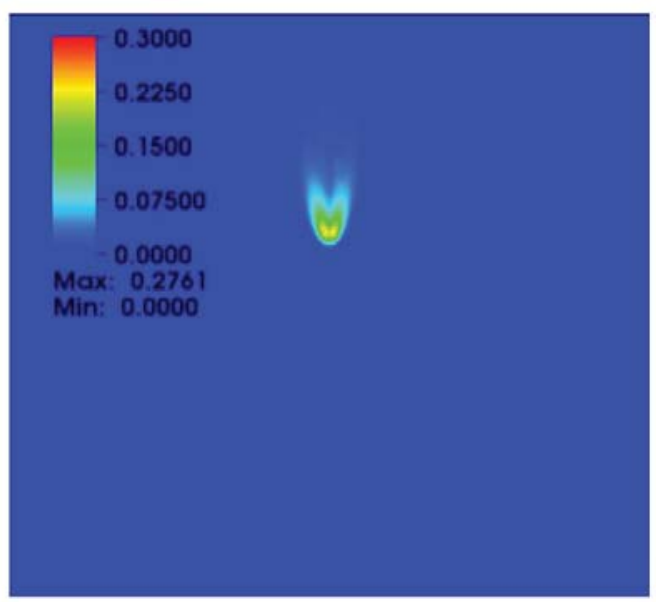

(f) LES: HVO

Figure 5.16: Gas phase fuel mass fraction of the evaporating case using different fuels at $0.0008 \mathrm{~s}$ for the RANS and the LES simulations. 


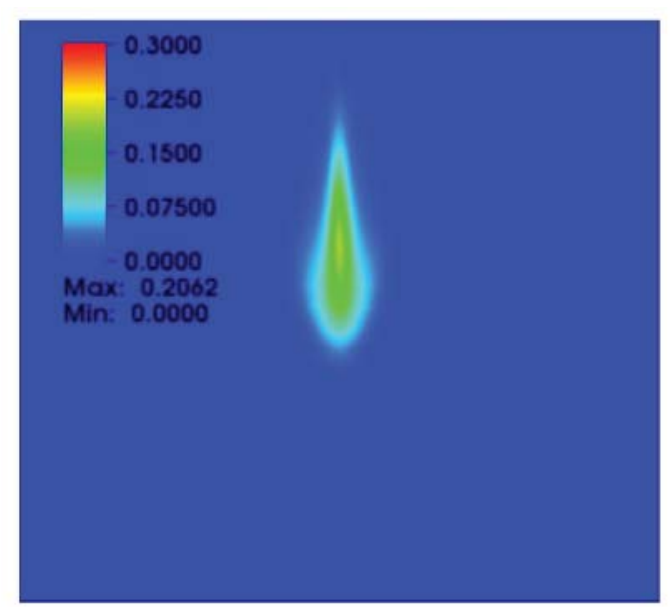

(a) RANS: HEP

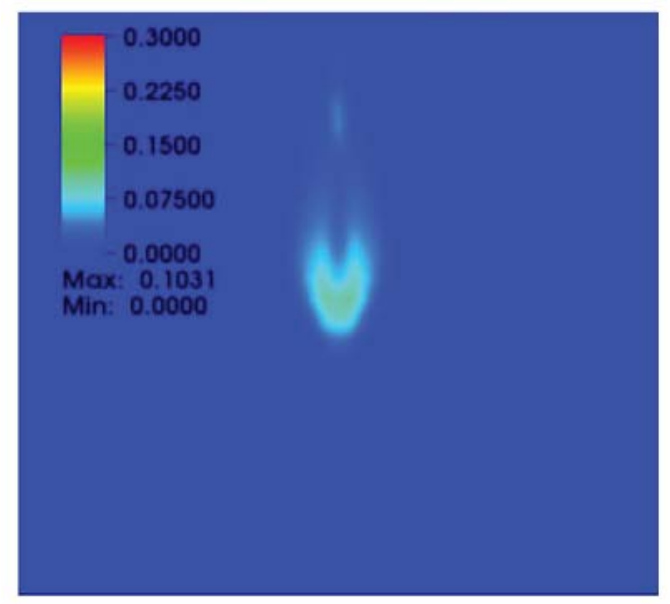

(c) RANS: EN 590

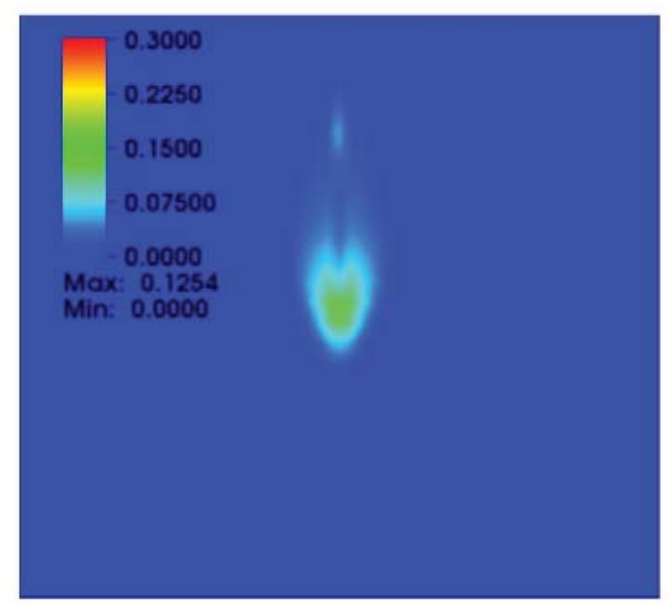

(e) RANS: HVO

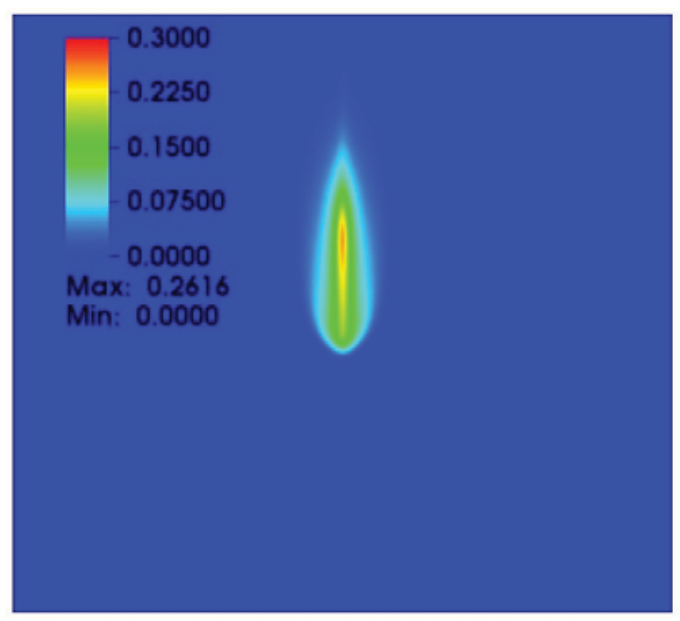

(b) LES: HEP

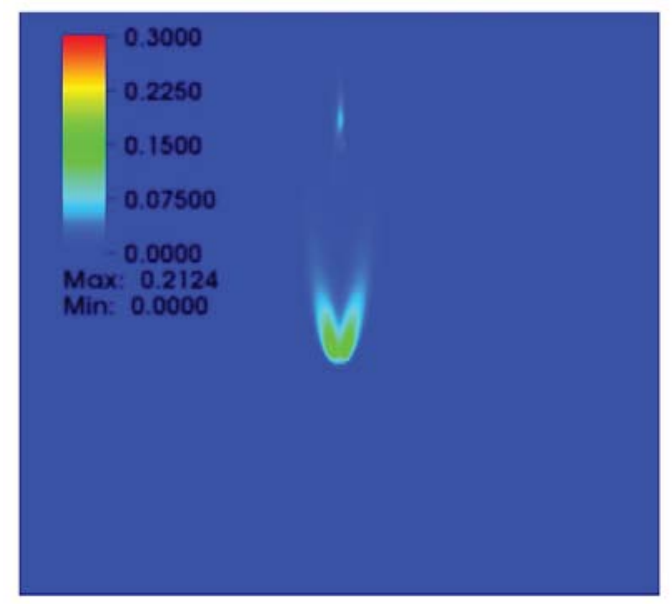

(d) LES: EN 590

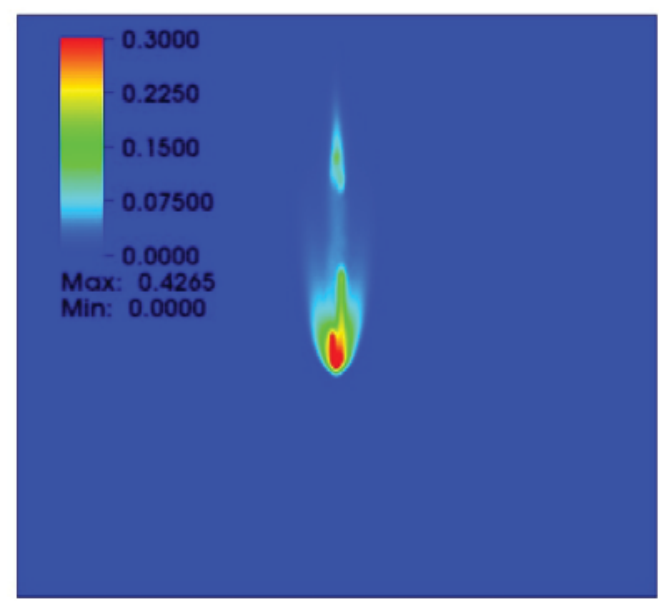

(f) LES: HVO

Figure 5.17: Gas phase fuel mass fraction of the evaporating case using different fuels at $0.0016 \mathrm{~s}$ for the RANS and the LES simulations. 
The fuel mass fractions on a cut plane through the spray axis at the injection time of $0.8 \mathrm{~ms}$ and $1.6 \mathrm{~ms}$ are shown in Figs. 5.16 and 5.17, respectively. Again, the plots are consistent with the evaporation rates of the three fuels; namely, n-heptane exhibits a considerably higher fuel vapor concentration than HVO and EN 590.

Additionally, in the LES, the liquid penetration of HVO and EN 590 are only slightly lower than their vapor penetration, which is consistent with the observations in the liquid phase fuel spray plots (Figs. 5.14 and 5.15) and the gas phase fuel mass fraction plots (Figs. 5.16 and 5.17). The liquid phase fuel sprays develop cone-like shapes near the tip in the RANS, and slim-stick shapes in the LES. The fatter fuel rich regions in the RANS are caused by the over-prediction of the jet spreading due to over-dissipation. Hence, there are more droplets downstream and aggregated near the spray tip in the LES, and it is harder to evaporate fully than the RANS. The larger penetrations in LES do not mean that the LES cases evaporate slower than the RANS cases. In fact, LES evaporates faster than RANS, which is consistent with the higher minimum temperature, $T_{\min }$, of the LES in Fig. 5.15. This is because the LES simulations capture more turbulence than the RANS simulations, and thus more heat is transferred from the hot gases to the cold fuel droplets in the LES.

Furthermore, it is shown in Fig. 5.1 that the vapor pressure of HVO is slightly greater than that of EN 590. As shown in Fig. 5.15, the LES exhibited an obvious faster evaporation of HVO than EN 590, while it is not so obvious for the RANS. The minimum temperature $T_{\min }$ of EN 590 is always smaller than that of HVO, and the difference observed in the LES 
is much greater than that in the RANS.

The cross-sectional SMDs for the evaporating cases are shown in Fig. 5.18 together with a more detailed view in the downstream region of the spray. Again, the behavior of EN 590 and $\mathrm{HVO}$ are similar because of their identical surface tension.

It is also seen that the droplet diameters predicted by the RANS simulations are larger than the LES. As discussed in Chapter 4, the larger the droplet, the smaller the liquid to air surface ratio, and, therefore, less momentum and heat exchange. The lower momentum exchange between the liquid and the gas in the RANS leads to the smaller gas velocity, as is illustrated in Fig. 5.19. The lower heat exchange in the RANS is also consistent with the lower $T_{\min }$, as shown in Fig. 5.15.

In addition, as is shown in the detailed plots in Fig. 5.18, the SMDs are larger at the spray tips. This phenomenon is referred to as the tip-clustering effect in Tanner and Boulouchos ${ }^{80}$ and is a consequence of enhanced drop coalescence at the spray tip. This is caused by tailwind interactions between subsequent droplets at the tip of the spray. This phenomenon has been confirmed in the study of Kihm et al. ${ }^{81}$ 

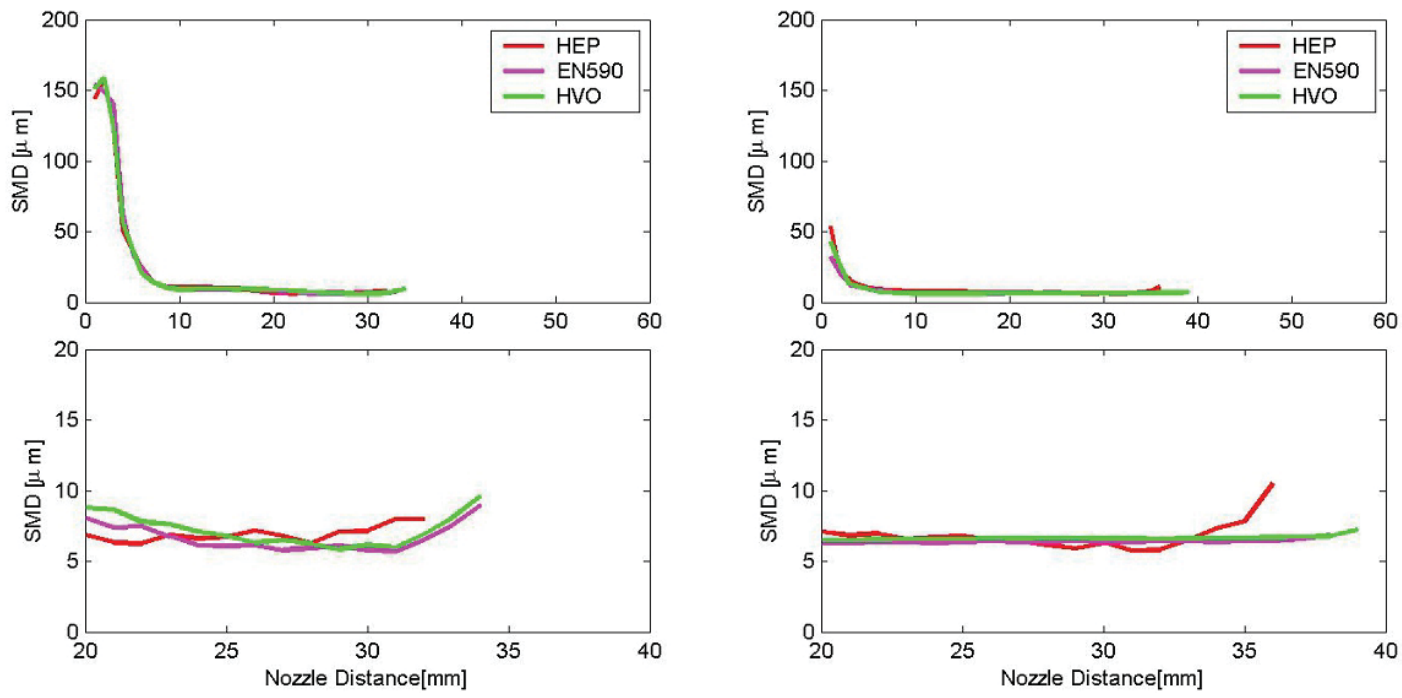

(a) RANS

(b) LES

Figure 5.18: Cross-sectional SMD of the evaporating cases at time $=0.0008 \mathrm{~s}$ for the RANS and LES simulations.
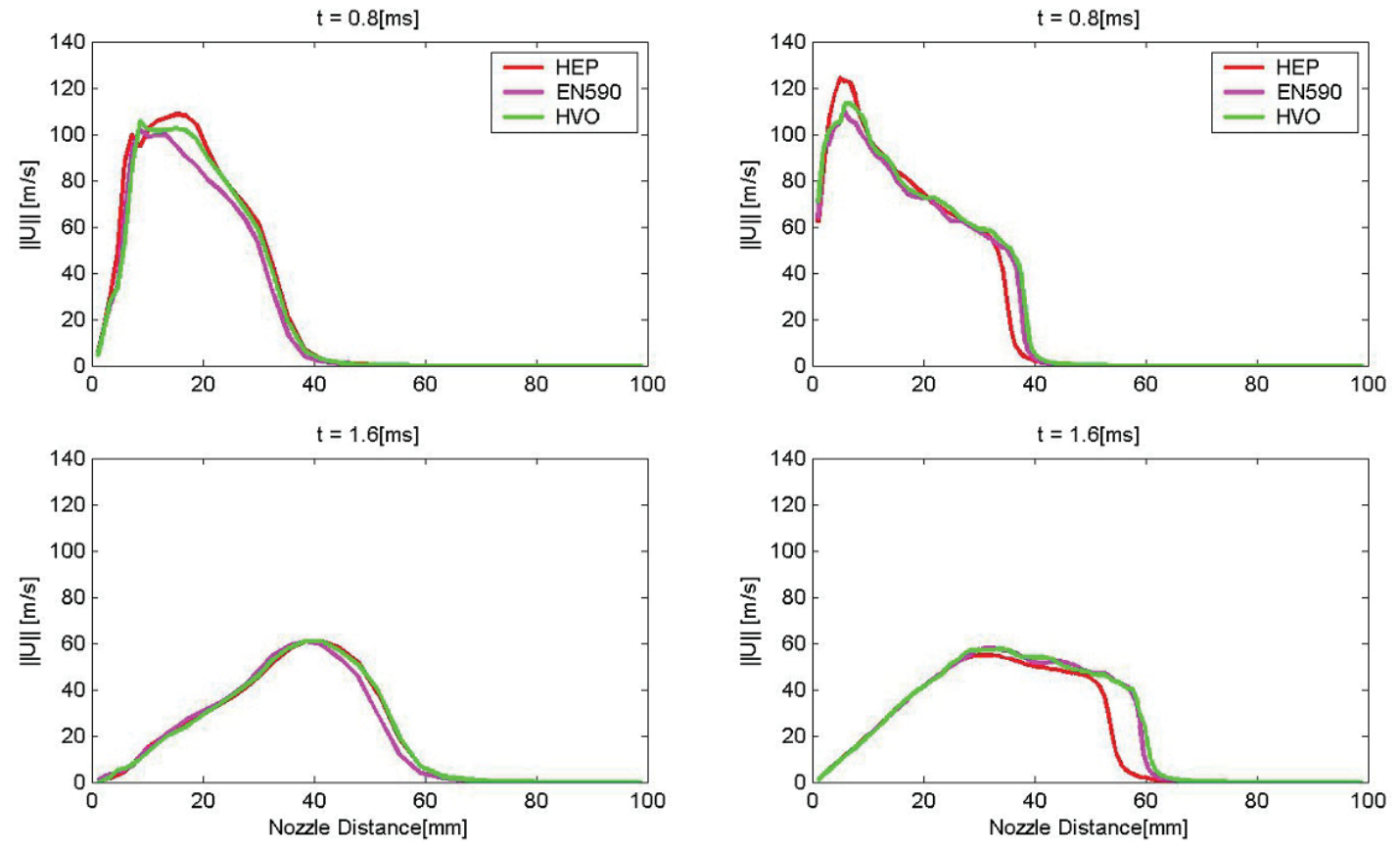

(a) RANS

(b) LES

Figure 5.19: Magnitude of axial gas velocity versus nozzle distance along the centerline of the evaporating cases at $0.0008 \mathrm{~s}$ and $0.0016 \mathrm{~s}$ for the RANS and LES simulations. 


\subsection{Conclusion}

For both, the non-evaporating cases and the evaporating cases, the EN 590 and HVO fuels exhibit very similar spray tip penetrations and droplet size. In the non-evaporating cases, the better stability of the HVO drops near the nozzle attributes the larger near nozzle SMDs in the LES. But this shortcoming of HVO can be avoided by adjusting the injection velocity. In the evaporating cases, HVO evaporates a little faster than EN 590. The similar or even better behavior of HVO, suggest that HVO can be used as an alternative fuel for EN 590 in the current diesel engines without changes to the injection system or engine components such as the combustion chamber or piston. 


\section{Future work}

As shown in this thesis, LES is mesh dependent, which is caused by the liquid phase submodels. ${ }^{82}$ The improved submodels, e.g. Schmidtą́r collision model with a secondary collision mesh, ${ }^{78}$ are expected to be introduced into OpenFOAM to decrease the mesh dependence of LES.

Additionally, in the spray regions, the high gradients of the velocity and vapor concentration also lead to the LES mesh dependence. Chapter 4 showed that the coarse mesh under-predicted the spray penetration, caused by the overestimation of the momentum diffusion. ${ }^{83}$ Refining the mesh is not practical due to the highly increased computation cost. Further investigations for avoiding mesh dependence without increasing the number of computation cells are required. One possibility might be the realization of the adaptive mesh refinements (AMR). ${ }^{84}$

In this thesis the comparisons between RANS and LES have been limited to non-reacting sprays. Reacting sprays, i.e., spray processes which consider chemical reactions, are also of prime interest, in particular to study pollution formation. In order to conduct such investigations, the chemical properties of HVO need to be known. Such investigations will be performed in a future project. 


\section{References}

[1] P. T. Williams and A. J. Baker, "Numerical simulations of laminar flow over a 3D backward-facing step," International Journal for Numerical Methods in Fluids, 1997.

[2] J. Smagorinsky, "General circulation experiments with the primitive equations," Monthly Weather Review, vol. 91, pp. 99-165, 1963.

[3] R. Peyret, Handbook of Computational Fluid Mechanics. London, UK: Academic Press, 1st ed., 1996.

[4] B. E. Launder and D. B. Spalding., Mathematical models of turbulence. London: Academic Press Inc. Limited., 1st ed., 1972.

[5] V. Yakhot, S. A. Orszag, S. Thangam, T. B. Gatski, and C. G. Speziale, "Development of turbulence models for shear flows by a double expansion technique," Physics of Fluids, vol. A 4(7), pp. 1510-1520, 1992.

[6] V. Yakhot and L. M. Smith, "The renormalization group, the $\varepsilon$-expansion and derivation of turbulence models," Journal of Scientific Computing, vol. 7, pp. 35-61, 1992.

[7] U. Schumann, "Realizability of Reynolds-stress turbulence models," Physics of Fluids, 1977.

[8] J. Blazek, Computational Fluid Dynamics: Principles and Applications. Oxford, UK: Elsevier Science Ltd, 1st ed., 2001.

[9] A. Raudkivi, Advanced Fluid Mechanics. New York, USA: Halsted Press, 1st ed., 1975.

[10] D. A. Anderson, J. C. Tannehill, and R. H. Pletcher, Computational Fluid Mechanics and Heat Transfer. USA: Hemisphere Publishing Corporation, 1st ed., 1984.

[11] J. O. Hinze, Turbulence. New York: McGraw-Hill, 2nd ed., 1975.

[12] N. Rott, "Note on the history of the Reynolds number," Annual Review of Fluid Mechanics, 1990.

[13] A. N. Kolmogorov, "The local structure of turbulence in compressible turbulence for very large reynolds numbers," Doklady Akademii nauk SSSR, vol. 30, 1941.

[14] A. N. Kolmogorov, "Dissipation of energy in a locally isotropic turbulence," Doklady Akademii nauk SSSR, vol. 32, 1941. 
[15] R. Mathieu and J. Scott, An Introduction to Turbulent Flow. Cambridge, UK: Cambridge University Press, 1st ed., 2000.

[16] S. B. Pope, Turbulent Flows. UK: Cambridge University Press, 1st ed., 2000.

[17] H. Tennekes and J. L. Lumley, A First Couse in Tubulence. USA: The MIT Press, 1st ed., 1972.

[18] B. F. Magnussen, "On the structure of turbulence and a generalized eddy dissipation concept for chemical reaction in turbulent flow," in 19th American Institute of Aeronautics and Astronautics Aerospace Science Meeting, (St. Louis, Missouri, USA), January 12-15, 1981.

[19] S. Yang, B. Peschke, and K. Hanjalic, "Measurement and modeling of diesel sprays," J. Engineering for Gas Turbines and Power, vol. 122, pp. 355-363, 2000.

[20] B. F. Armaly, F. Durst, J. C. F. Pereira, and B. Schonung, "Experimental and theoretical investigation of backward-facing step flow," Journal of Fluid Mechanics, 1983.

[21] O. Reynolds, "On the dynamical theory of incompressible viscous fluids and the determination of the criterion," Philosophical Transactions of the Royal Society of London, vol. A 186, pp. 123-164, 1895.

[22] G. J. Wagner and W. K. Liu, "Turbulence simulation and multiple scale subgrid models," Computational Mechanics, 2000.

[23] J. Boussinesq, "Essai sur la théorie des eauzx courantes," Mémoires présentés par divers savants, L extquoteright Acad. des Sci. Inst. Nat. France, vol. 23, p. 46, 1877.

[24] W. P. Jones and B. E. Launder, "The prediction of laminarization with a two-equation model of turbulence," International Journal of Heat and Mass Transfer, vol. 15, pp. 301-314, 1972.

[25] B. E. Launder and B. I. Sharma, "Application of the energy dissipation model of turbulence to the calculation of flow near a spinning disc," Letters in Heat and Mass Transfer, vol. 1, no. 2, pp. 131-138, 1974.

[26] R. B. Stull, An Introduction to Boundary Layer Meteorology. Springer, 1st ed., 1988.

[27] E. Garnier, P. Sagaut, and N. Adams, Large Eddy Simulation for Compressible Flows. Springer, 2009.

[28] S. Menon, P. K. Yeung, and W. W. Kim, "Effect of subgrid models on the computed interscale energy transfer in isotropic turbulence," Computers \& Fluids, 1996. 
[29] A. Yoshizawa and K. Horiuti, "A statistically-derived subgrid-scale kinetic energy model for the large-eddy simulation of turbulent flows," Journal of the Physical Society of Japan, 1985.

[30] OpenFOAM, "The open source CFD toolbox." OpenCFD Ltd., 2004-2008. http://www.openfoam.org.

[31] K. Liu and D. C. Haworth, "Large-eddy simulation for an axisymmetric pistoncylinder assembly with and without swirl," (Detroit), International Multidimensional Engine Modeling User's Group Meeting, April 19th 2009.

[32] OpenFOAM Programmer's Guide, 1.5 ed., July 2008.

[33] OpenFOAM User Guide, 1.5 ed., July 2008.

[34] H. Jasak, Error analysis and estimation in the Finite Volume method with applications to fluid flows. PhD thesis, Imperial College, University of London, 1996.

[35] R. Eymard, T. R. Galloüt, and R. Herbin, The Finite Volume Method Handbook of Numerical Analysis, vol. VII. 2000.

[36] R. Courant, K. Friedrichs, and H. Lewy, "On the partial difference equations of mathematical physics," 1967.

[37] R. B. Piccinini, EULERIAN-LAGRANGIAN SIMULATION OF A TURBULENT EVAPORATING SPRAY. PhD thesis, Campo Montenegro, SP - Brazil, 2011.

[38] V. Subramaniam, COMPUTATIONAL ANALYSIS OF BINARY-FLUID HEAT AND MASS TRANSFER IN FALLING FILMS AND DROPLETS. PhD thesis, Georgia Institute of Technology, 2008.

[39] S. V. Patankar and D. B. Spalding, "A calculation procedure for heat, mass and momentum transfer in parabolic flows," Int. J. Heat Mass Transfer, vol. 15, 1972.

[40] R. I. Issa, "Solution of the implicitly discretised fluid flow equations by operatorsplitting," Journal of Computational Physics, vol. 62, pp. 40-65, 1986.

[41] H. K. Versteeg and W. Malalasekera, An Introduction to Computational Fluid Dynamics: The Finite Volume Method. Prentice Hall, 1995.

[42] Shashank, J. Larsson, and G. Iaccarino, "A co-located incompressible NavieríCStokes solver with exact mass, momentum and kinetic energy conservation in the inviscid limit," Journal of Computational Physics, vol. 299, p. 4425ÍC4430, 2010.

[43] C. Rhie and W. Chow, "Numerical study of the turbulent flow past an airfoil with trailing edge separation," AIAA Journal, vol. 21, no. 11, p. 1525íC32, 1983. 
[44] V. Vuorinen and M. Larmi, "Large-eddy simulation on the effect of droplet size distribution on mixing of passive scalar in a spray," SAE Paper 2008-01-0933, 2008.

[45] Y. Gong and F. Tanner, "Comparison of RANS and LES models in the laminar limit for a flow over a backward-facing step using OpenFOAM," (Detroit), International Multidimensional Engine Modeling User's Group Meeting, April 19th 2009.

[46] S. Balachandar and J. Eaton, "Turbulent dispersed multiphase flow," Annual Review of Fluid Mechanics, vol. 42, pp. 111-133, 2010.

[47] A. A. Amsden, P. J. O'Rourke, and T. D. Butler, KIVA-II: A Computer Program for Chemically Reactive Flows with Sprays. Los Alamos National Laboratory, Los Alamos, New Mexico 87545, May 1989.

[48] Y. Gong, O. Kaario, A. Tilli, M. Larmi, and F. Tanner, "A computational investigation of hydrotreated vegetable oil sprays using RANS and a modified version of the RNG k-epsilon model in OpenFOAM,” SAE Paper 2010-01-0739, 2010.

[49] F. P. Krrholm, Numerical Modelling of Diesel spray Injection, Turbulence Interaction and Combustion. $\mathrm{PhD}$ thesis, Chalmers University of Technology, Sweden, 2008.

[50] A. Williams, Combustion of sprays of liquid fuels. London: Elek Science, 1976.

[51] F. X. Tanner, "Development and validation of a cascade atomization and drop breakup model for high-velocity dense sprays," Atomization and Sprays, vol. 14, pp. 211-242, 2004.

[52] V. G. Levich, Physicochemical Hydrodynamics. Englewood Cliffs, New Jersey: Prentice-Hall, 1962.

[53] G. Pizza, Y. M. Wright, G. Weisser, and K. Boulouchos, "Evaporating and nonevaporating diesel spray simulation: comparison between the ETAB and wave breakup model," International Journal of Vehicle Design, vol. 45, 2007.

[54] P. J. O'Rourke and A. A. Amsden, "The tab method for numerical calculation of spray droplet breakup," Tech. Rep. 872089, SAE Paper, 1987.

[55] A. B. Liu and R. D. Reitz, "Mechanisms of air-assisted liquid atomization," Atomization and Sprays, vol. 3, pp. 55-75, 1993.

[56] F. P. Kärrholm, Numerical Modelling of Diesel Spray Injection, Turbulence Interaction and Combustion. PhD thesis, Chalmers University of Technology, Göteborg, Sweden,, 2008.

[57] C. Kralj, Numerical Simulation of Diesel Spray Processes. PhD thesis, Imperial College, 1995. 
[58] “FLUENT 6.1 user’s guide.” Fluent Inc., Jan 2003.

[59] M. M. El Wakil, O. A. Uyehara, and P. O. Myers, "A theoretical investigation of the heating-up period of injected fuel droplets," NACA TN 3179, 1954.

[60] W. E. Ranz and W. R. Marshall, "Evaporation from drops," Chem. Eng. Prog., vol. 48, pp. 141-146, 1952.

[61] C. Baumgarten, Mixture formation in internal combustion engines. Springer Verlag, 2006.

[62] N. Ashgriz and J. Y. Poo, "Coalescence and separation in binary collisions of liquid drops," Journal of Fluid Mechanics, 1990.

[63] P. R. Brazier-Smith, S. G. Jennings, and J. Latham, "The interaction of falling water droplets: coalescence.," Proceedings of the Royal Society of London A, vol. 326, p. 393íC408, 1972.

[64] S. L. Post and J. Abraham, "Modelingthe outcome of dropĺcdrop collisions in diesel sprays," International Journal of Multiphase Flow, vol. 28, 2002.

[65] P. J. OąrRourke, Collective drop effects on vaporizing liquid sprays. PhD thesis, Mechanical and Aerospace Engineering, Princeton University, USA, 1981.

[66] N. Nordin, Complex Chemistry Modeling of Diesel Spray Combustion. PhD thesis, Chalmers University of Technology, 2001.

[67] S. Lain, D. Bröder, M. Sommerfeld, and M. Göz, "Modeling hydrodynamics and turbulence in a bubble column using the Euler-Lagrange procedure," International journal of multiphase flow, vol. 28, pp. 1381-1407, 2002.

[68] V. Vuorinen, LES of Certain Droplet Size Effects in Fuel Sprays. PhD thesis, Aalto University, Finland, 2010.

[69] C. Crowe, T. Chung, and T. Troutt, "Particle mixing in free shear flows," Prog. Energy Combust. Sci., vol. 14, p. 1711́C194, 1988.

[70] H. Hiroyasu and T. Kadota., "Fuel droplet size distribution in diesel combustion chamber.," SAE Paper 740715, 1974.

[71] H. Ko $\beta$, D. Brüggemann, A. Wiartalla, H. Bäcker, and A. Breuer, "Results from fuel/air ratio measurements in an n-heptane injection spray," tech. rep., IDEA periodic report, RWTH Aachen, 1992.

[72] S. Pope, Turbulent Flows. England: Cambridge University Press, 2008.

[73] K. Jaguś, LARGE EDDY SIMULATION OF FUEL INJECTION AND SPRAY COMBUSTION IN AN ENGINE ENVIRONMENT. PhD thesis, Brunel University, 2009. 
[74] M. Li and C. Garrett, "The relationship between oil droplet size and upper ocean turbulence," Marine Pollution Bulletin, vol. 36, no. 12, pp. 961-970, 1998.

[75] A. Devesa, J. Moreau, and T. Poinsot, "Large eddy simulations of jet/tumble interaction in a GDI model engine flow," SAE Paper 2004-01-1997, 2004.

[76] H. Aatola, M. Larmi, T. Sarjovaara, and A. Affiliations, "Hydrotreated Vegetable Oil (HVO) as a renewable diesel fuel: Trade-off between NOx, particulate emission, and fuel consumption of a heavy duty engine," SAE International Journal of Engines, vol. 1, no. 1, pp. 1251-1262, 2009.

[77] M. Endisch, U. Balfanz, M. Olschar, and T. Kuchling, "Vegetable oil hydrotreating for production of high quality diesel components," (Berlin, Germany), DGMK Conference, Future Feedstocks for Fuels and Chemicals, Sep 29th-Oct 1st 2008.

[78] D. P. Schmidt and P. K. Senecal, "Improving the numerical accuracy of spray simulations," SAE Paper 2002-01-1113, 2002.

[79] C. Yaws, ed., Chemical Properties Handbook. McGraw-Hill, 1999.

[80] F. X. Tanner and K. Boulouchos, "A computational investigation of the sprayinduced flow and its influence on the fuel distribution for continuous and intermittent DI-diesel sprays," SAE Paper 960631, 1996.

[81] K. D. Kihm, D. P. Terracina, and J. A. Caton, "Spray-tip droplet SMDs of intermittent high-pressure sprays of diesel fuel compared with coal-water slurry sprays," Journal of the Institute of Energy, vol. 68, 1995.

[82] R. Aneja and J. Abraham, "How far does the liquid penetration in diesel engine: computed results vs. measurements," Combustion Science and Technology, 1998.

[83] Q. Xue and S.-C. Kong, "Applications of local adaptive mesh refinement scheme for engine spray simulations," (Detroit, MI), International Multidimensional Engine Modeling User's Group Meeting at the SAE Congress, April 132008.

[84] T. Plewa, T. Linde, and V. G. Weirs, eds., Adaptive Mesh Refinement-Theory And Applications. Springer, 2005.

[85] H. D. Baehr, Thermodynamik. Berlin: Springer-Verlag, eighth ed., 1992.

[86] C. Bai and A. D. Gosman, "Mathematical modeling of wall films formed by impinging sprays," SAE Paper 960626, 1996.

[87] B. Baldwin and T. Barth in AIAA Paper 91-0610, (Reno, NV), AIAA 29th Aerospace Sciences Meeting, 1991. 
[88] J. Bardina, J. H. Ferziger, and W. C. Reynolds, "Improved subgrid sclae models for large eddy simulation," AIAA Paper 80-1357, 1980.

[89] I. E. Barton, "Comparison of simple- and piso-type algorithms for transient flows," International Journal for Numerical Methods in Fluids, 1998.

[90] J. Barata, A. Cometti, A. Mendes, and A. Silva, "Numerical simulation of an array of droplets through a crossflow," (Reno, NV), 40th AIAA Aerospace Sciences Meeting \& Exhibit, AIAA 2002-0872, January 2002.

[91] F. Bedford and C. Rutland, "Mechanisms of $N O_{x}$ reduction from water injection in a DI diesel engine," in Proc. ICE Division of ASME, (Columbus, IN), April 1999.

[92] J. H. Ferziger, Computational Methods for Turbulent, Tansonic, and Viscous Flows, ch. Higher-Level Simulation of Turbulent Flows, pp. 93-182. Hemisphere Publishing Corp., 1983.

[93] C. T. Crowe, Multiphase Flow Handbook. USA: CRC Press, 1st ed., 2006.

[94] H. Schlichting, Boundary-Layer Theory. New York: McGraw-Hill, sixth ed., 1968.

[95] J. O. Hirschfelder, C. F. Curtiss, and R. B. Bird, Molecular Theory of Gases and Liquids. New York: Wiley, 1st ed., 1954.

[96] J. Boussinesq, "théorie de l'écoulement tourbillant," Mem. Pre. par. div. Sav., vol. 23, pp. 46-50, 1877.

[97] T. D. Butler, L. D. Cloutman, J. K. Dukowicz, and J. D. Ramshaw, "Multidimensional numerical simulation of reactive flow in internal combustion engines," Prog. Energy Combust. Sci., vol. 7, pp. 293-315, 1981.

[98] P. Carlsson, “Tutorial dieselfoam.” http://www.tfd.chalmers.se/ hani/kurser/OS_CFD_2008/PerCarlsson/PC_Tutorial_dieselFoam_peered_NL_HN.pdf, February 17, 2009.

[99] C. T. Crowe and I. Gillandt, "Turbulent modulation of fluid-particle flowsíca basic approach," in Third International Conference on Multiphase Flows, (Lyon, France), 1998.

[100] J. W. Deardorff, "A numerical study of three-dimensional turbulent channel flow at large reynolds numbers," J. Fluid Mech, vol. 41, pp. 453-480, 1970.

[101] J. H. Ferziger and M. Peric, Computational Methods for Fluid Dynamics. Springer, 3rd ed., 2001.

[102] G. Gouesbet and A. Berlemont, "Eulerian and Lagrangian approaches for prediction the behavior of discrete particles in turbulent flows," Progress in Energy and Combustion Science, vol. 25, pp. 133-159, 1999. 
[103] C. Hirt, A. A. Amsden, and J. L. Cook., "An arbitrary lagrangian-eulerian computing method for all flow speeds," J. Comput. Phys., 1974.

[104] X. Huai, R. D. Joslin, and U. Piomelli, "Large-eddy simulation of transition to turbulence in boundary layers," Theoret. Comput. Fluid Dynamics, 1997.

[105] J. R. Khan, Fog cooling, wet compression and droplet dynamics in gas turbine compressors. PhD thesis, University of New Orleans, 2009.

[106] S. Lain and M. Sommerfeld, "Turbulence modulation in dispersed tow-phase flow laden with solids from a Lagrangian perspective," Heat and Fluid Flow, vol. 24, pp. 616-625, 2003.

[107] A. H. Lefebvrer, Gas Turbine Combustion. USA: CRC, 2nd ed., 1998.

[108] S. Patankar, Numerical Heat Transfer and Fluid Flow. USA: Hemisphere Publishing Corporation, 1981.

[109] Z. Han, S. Parrish, P. Farrell, and R. Reitz, "Modeling atomization processes of pressure-swirl hollow-cone fuel sprays applications," Atomization and Sprays, vol. 7, pp. 663-684, 1997.

[110] L. Fan, G. Li, Z. Han, and R. Reitz, "Multidimensional modeling of spray atomization and air-fuel mixing in a direct-injection spark-ignition engine," SAE Paper 1999-01-0175, 1999.

[111] L. Richardson, Proc. Roy. Soc. A 110. 1922.

[112] B. Hu and C. J. Rutland, "Flamelet modeling with les for diesel engine simulations," SAE Paper 2006-01-0058, 2006.

[113] R. Jhavar and C. J. Rutland, "Using large eddy simulations to study mixing effects in early injection diesel engine combustion," SAE Paper 2006-01-0871, 2006.

[114] P. Sagaut, Large Eddy Simulation for Incompressible Flows. Springer, 2 ed., 2002.

[115] V. Vuorinen, H. Hillamo, O. Kaario, M. Nuutinen, M. Larmi, and L. Fuchs, "Effect of droplet size and breakup on spray shape: A priori study using large-eddy simulation," Turbulence. Heat and Mass Transfer, 2009.

[116] G. Weisser, F. Tanner, and K. Boulouchos, "Towards CRFD-simulation of large diesel engines: Modeling approaches for key processes," in Proc. $3^{\text {rd }}$ Int. ICE Conference, (Capri, Italy), September 1997.

[117] G. Weisser, F. Tanner, and K. Boulouchos, "Modeling of ignition and early flame development with respect to large diesel engine simulation," SAE Paper 981451, 1998. 
[118] G. Weisser, F. Tanner, and K. Boulouchos, "Modeling of ignition and early flame development with respect to large diesel engine simulation," SAE Transactions: Journal of Fuels and Lubricants, vol. 107, no. 4, pp. 802-811, 1999.

[119] G. Weisser, F. Tanner, K. Boulouchos, J. Kramer, and R. Holtbecker, "Integrating CRFD-simulations into the development process of large diesel engines: A status report,' CIMAC Paper no 5.09, CIMAC '98, Copenhagen, May 1998.

[120] G. Weisser, F. Tanner, and K. Boulouchos, "A computational study of the effect of nozzle geometry on nitric oxide formation under medium-size diesel engine conditions," in Proc. Second Symposium Towards Clean Diesel Engines, (Paul Scherrer Institute (PSI), Villigen, Switzerland), May 1998.

[121] G. Weisser, Modeling of Combustion and Nitric Oxide Formation for Medium-Size Diesel Engines: Zero and Three-Dimensional Approaches. PhD thesis, Swiss Federal Institute of Technology (ETH). In preparation.

[122] H. Jasak, Error Analysis and Estimation for the Finite Volume Method with Applications to Fluid Flows. PhD thesis, Imperial College of Science, Technology and Medicine, 1996. OpenFOAM.

[123] D. Wickman, P. Senecal, and R. Reitz, "Diesel engine combustion chamber geometry optimization using genetic algorithms and multi-dimensional spray and combustion modeling," SAE Paper 2001-01-0547, 2001.

[124] D. D. Wickman, HSDI Diesel Engine Combustion Chamber Geometry Optimization. $\mathrm{PhD}$ thesis, University of Wisconsin-Madison, 2003.

[125] P. R. Wieber, "Calculated temperature histories of vaporizing droplets to the critical point," AIAA J., vol. 1(12), pp. 2764-70, 1963.

[126] D. C. Wilcox, Turbulence Modeling for CFD. La Canada, CA: DCW Industries, 1993.

[127] F. A. Williams, "On the assumptions underlying droplet vaporization and combustion theories," J. Chem. Phys., vol. 33(1), pp. 133-44, 1960.

[128] F. A. Williams, Combustion Theory. Menlo Park, CA: Benjamin/Cummings Publishing, 2nd ed., 1985.

[129] D. G. Wilson, The design of high-efficiency turbomachinery and gas turbines. Cambridge, Massachusetts: The MIT Press, 1st ed., 1984.

[130] F. Wirbeleit, C. Enderle, W. Lehner, A. Raab, and K. Binder, "Stratified diesel fuelwater-diesel fuel injection combined with EGR - the most efficient in-cylinder NOx and PM reduction technology," SAE Paper 972962, 1997. 
[131] A. Wolff, R. Müller, and K. Boulouchos, "A computational investigation of unsteady heat flux through an i.c. engine cylinder wall including soot layer dynamics," SAE Paper 970063, 1997.

[132] K.-J. Wu, D. A. Santavicca, F. V. Bracco, and A. Coghe, "LDV measurements of drop velocity in diesel-type sprays," AIAA J., vol. 22, no. 9, 1984.

[133] C.-T. Wu, J. Ferziger, and D. Chapman, "Simulation and modeling of homogeneous compressed turbulence," Dept. of Mechanical Engineering Technical Report No. TF21, Stanford University, 1985.

[134] J. Xin, D. Montgomery, Z. Han, and R. D. Reitz, "Multidimensional modeling of combustion for a six-mode emissions test cycle on a DI diesel engine," Journal of Engineering for Gas Turbines and Power, vol. 119, pp. 683-691, 1997.

[135] V. Yakhot and S. A. Orszag, "Renormalization group analysis of turbulence. I. Basic theory," Journal of Scientific Computing, vol. 1, pp. 3-51, 1986.

[136] J. Yang and J. K. Martin, "Approximate solution — one-dimensional energy equation for transient compressible, low Mach number turbulent boundary layer flows," J. Heat Transfer, vol. 111, pp. 619-24, 1989.

[137] Y. Yi and R. D. Reitz, "A numerical model for jet primary breakup," in Proc. 15th ILASS-Americas Annual Conference, (Madison, Wisconsin), May 2002.

[138] Y. Yi and R. D. Reitz, "Modeling the effect of primary atomization on diesel engine emissions," SAE Paper 2003-01-1041, 2003.

[139] Y. Yue, C. F. Powell, R. Poola, J. Wang, and J. K. Schaller, "Quantitative measurements of diesel fuel spray characteristics in the near-nozzle region using X-ray absorption," Atomization and Sprays, vol. 11, no. 4, pp. 471-490, 2001.

[140] A. J. Yule and A. P. Watkins, "Measurement and modeling of diesel sprays," Atomization and Sprays, vol. 1, pp. 441-465, 1991.

[141] A. J. Yule and D. G. Salters, "A conductivity probe technique for investigating the breakup of diesel sprays," Atomization and Sprays, vol. 4, pp. 41-63, 1994.

[142] A. Yule, A. Dalli, and K. Yeong, "Transient cavitation and separation in a scaled-up model of a VCO orifice," in Proc. 14th ILASS-Europe Annual Conference, (Manchester, England), pp. 230-235, July 1998.

[143] L. I. Zaichik and V. M. Alipchenkov, "A kinetic model for the transport of arbitrarydensity particles in turbulent shear flows," in Proc. turbulence and Shear Flow Phenomena 1, (Sta. Barbara CA, USA), 1998. 
[144] S. Zanforlin, R. D. Reitz, and R. Gentile, "Studying the roles of kinetics and turbulence in the simulation of diesel combustion by means of an extended characteristictime-model," SAE Paper 1999-01-1177, 1999.

[145] S. Zhang and C. Rutland, "Premixed flame effects on turbulence and pressure-related terms," Combustion and Flame, vol. 102, pp. 447-461, 1995. 



\section{A Computation Tool: OpenFOAM}

The numerical computations in this study are done by OpenFOAM (Open Field Operation and Manipulation), an open source Computational Fluid Dynamics (CFD) code with flexible and extendable $\mathrm{C}++$ libraries, using the finite volume method to solve systems of partial differential equations.

In this work, several user defined libraries and applications are developed and inserted into OpenFOAM. The details will be shown as following.

\section{A.1 New dieselSpray Submodels}

\section{A.1.1 IDSD: power law}

The initial parcel size distribution (IDSD), called the power law in Eq. 4.2, is realized as one probability distribution function power in the library, thermophysicalModels.

\$FOAM_SRC/thermophysicalModels/pdfs/power

Here, FOAM_SRC is the alias of the folder OpenFOAM-1.5/src.

The parameters of the power law are given in the file \$Casedir/constant/sprayProperties as the pdfType of IDSD, i.e. the dropletPDF of the injector, hollowConeInjector.

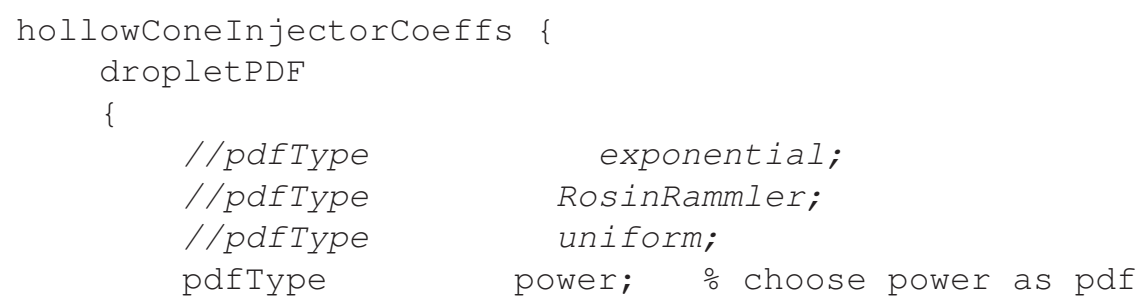




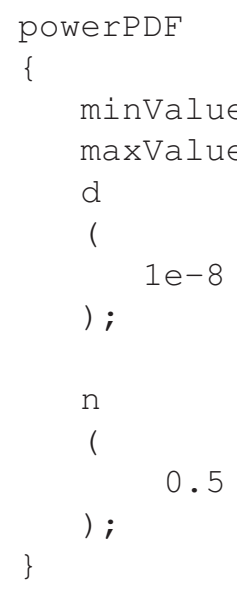

\section{A.1.2 Atomization Model: Levich}

In OpenFOAM, the Levich model in Sec. 4.1 is realized as a new atomization model in the dieselSpray class as following,

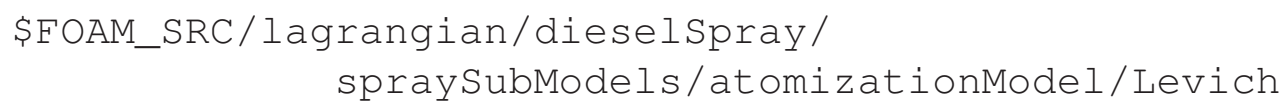

In this folder, the file Levich.C calculates the breakup length, 1BU, as in Eq. (4.3), where Clambda denotes the constant $C_{\lambda}$, rhoFuel is the fuel density $\rho_{l}$, rhoAverage equals the gas phase density $\rho_{g}$ and p.d() documents the drop diameter $d_{0}$ of this parcel.

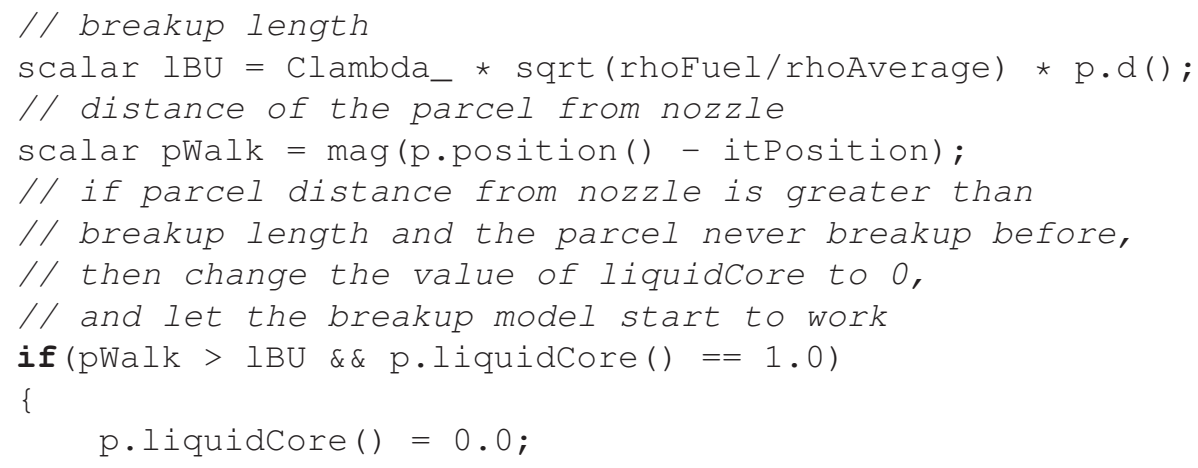


One calls the atomization model and the breakup model together, and the penetration curve will show a kink between this two stages as in Fig. A.1. To smooth the penetration curve, a small change of the calling order of the two processors, breakup.updateParcelProperties(...) and breakup().breakupParcel(...) are done in the file,

diesel Spray/spray/sprayOps.C.

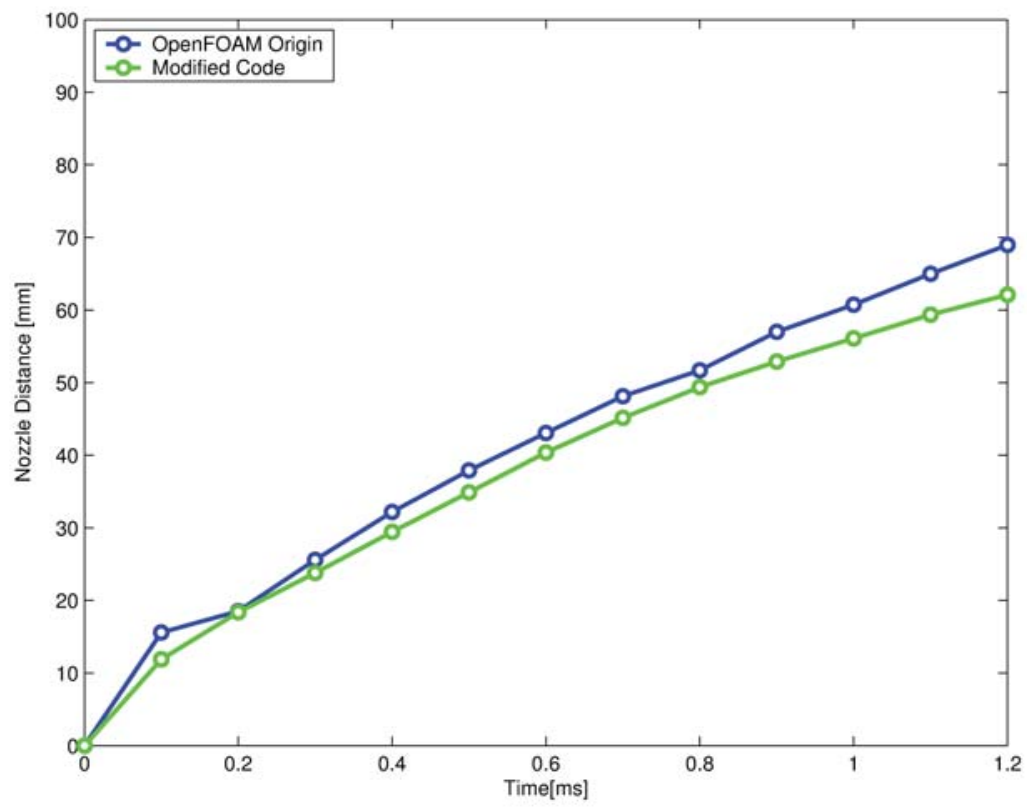

Figure A.1: Penetration curve of one tuning case: the blue line with the kink is the result of the OpenFOAM origin code, and the red line is the result of the modified code

The change is done as following, and the penetration curve is smoothed as shown in Fig. A.1.

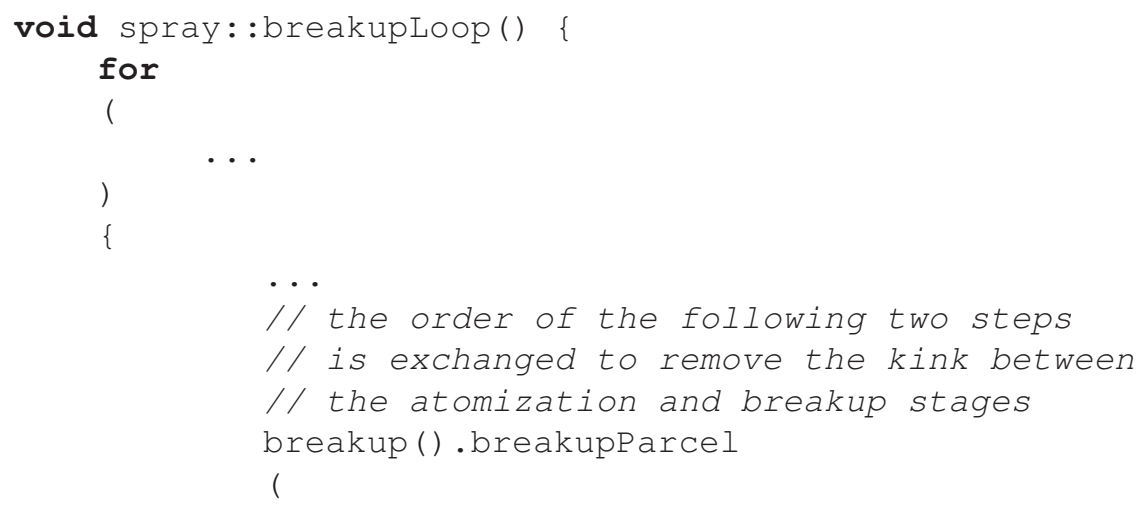




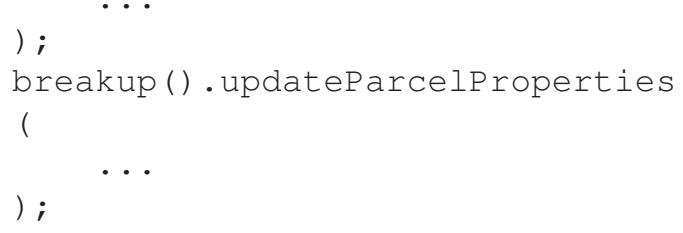

\section{A.1.3 Breakup Model: CAB}

The CAB model introduced in Sec. 4.1 is realized as one of the breakup models of the library dieselSpray.

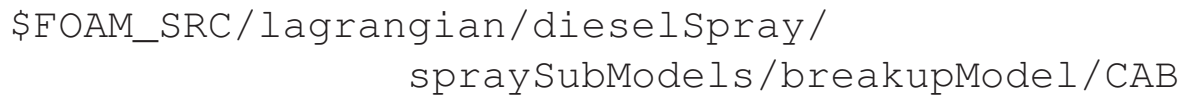

In the above folder CAB, there are two files. One is the CAB.H file, which declares the new parameters in the constructor of the class CAB. Refer to Eq. 4.6, k1_, k2_and k3_represent $k_{1}, k_{2}$ and $k_{3}$ separately, WeCrit_is $W e_{C r i t}$, WeTransition1 is $W e_{b, s}$ and WeTransition2 is $W e_{s, c}$. The value of $k_{1}, W e_{C r i t}, W e_{b, s}$ and $W e_{s, c}$ are given in the file \$casedir/constant/sprayProperties. The values of $k_{2}$ and $k_{3}$ are decided by the connection points of the three segmented curves in Eq. 4.6, and the definitions are given in another file CAB.C as following:

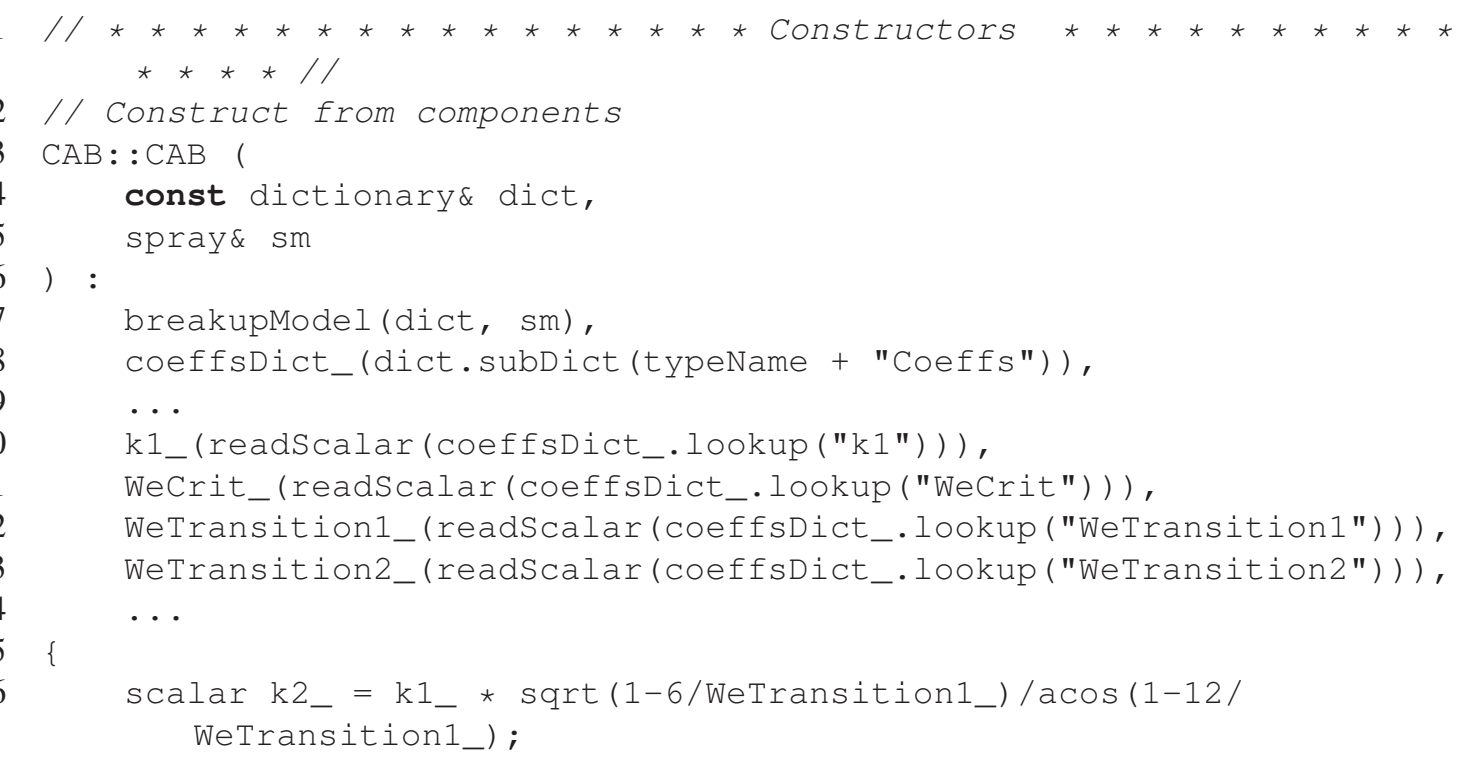




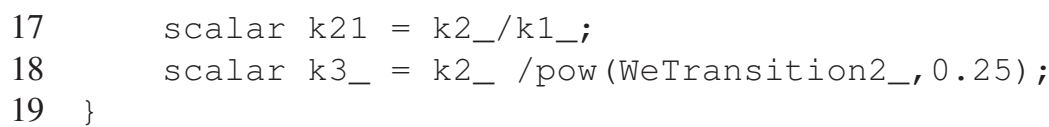

The coding of the CAB breakup model is based on the OpenFOAM defined ETAB breakup model. The biggest difference between the coding of these two breakup models is the calculation of Kbr, i.e. the droplet frequency $K_{b u}$ in Eq. 4.6.

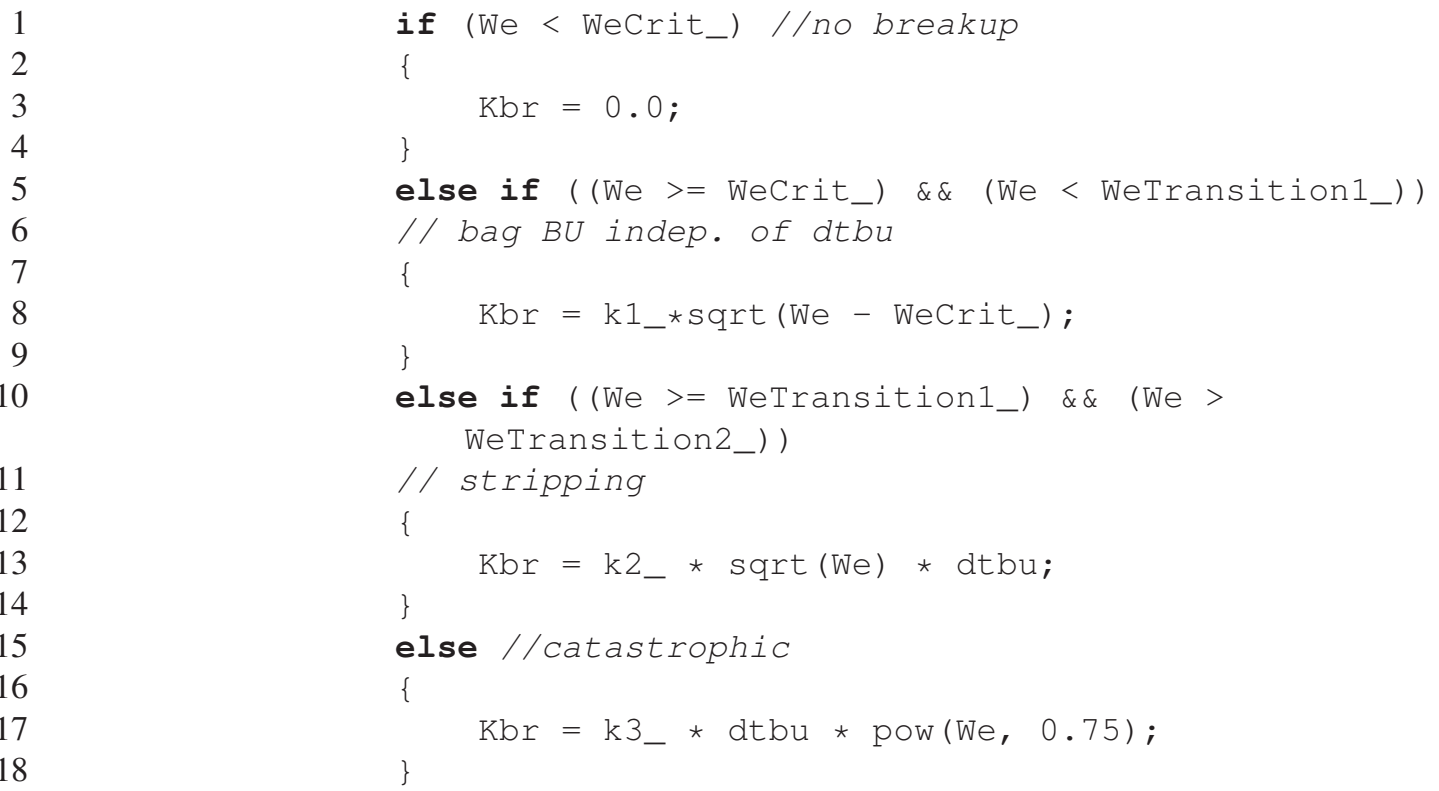

\section{A.2 dieselLES: diesel spray LES solver}

The OpenFOAM application dieselFoam resolves the diesel spray and combustion using RANS. However, in OpenFOAM, the relative solver using LES is not available. To solve the diesel spray using LES, a new solver dieselLES is developed based on the RANS diesel spray solver dieselFoam and the LES solver Xoodles which resolve the the compressible premixed/partially-premixed combustion with LES using the b-Xi two-equation model.

Noticed that the governing equations of the solver dieselFoam is contained in another solver dieselEngineFoam. Similarly, a new folder dieselEngineLES is generated as the folder dieselEngineFoam. 
First, the new solver dieselLES is created by coping all files in the solver dieselFoam.

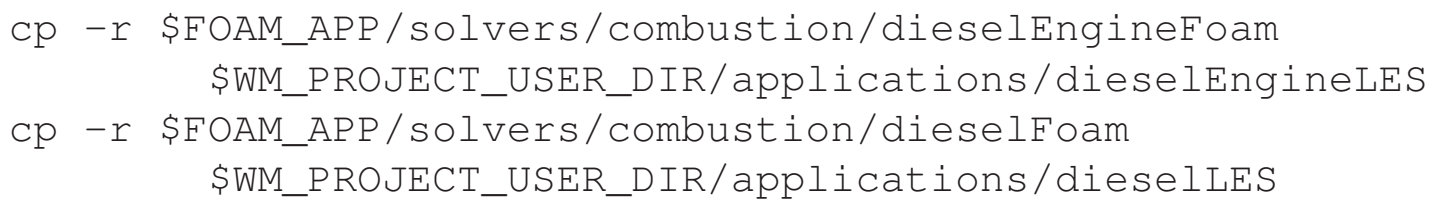

And then, all the strings, dieselEngineFoam and dieselFoam, are changed to dieseIEngineLES and dieselLES separately.

The dieselLES/Make/option file are modified for recompilation. The first change is resource the turbulence library from RAS to LES as following:

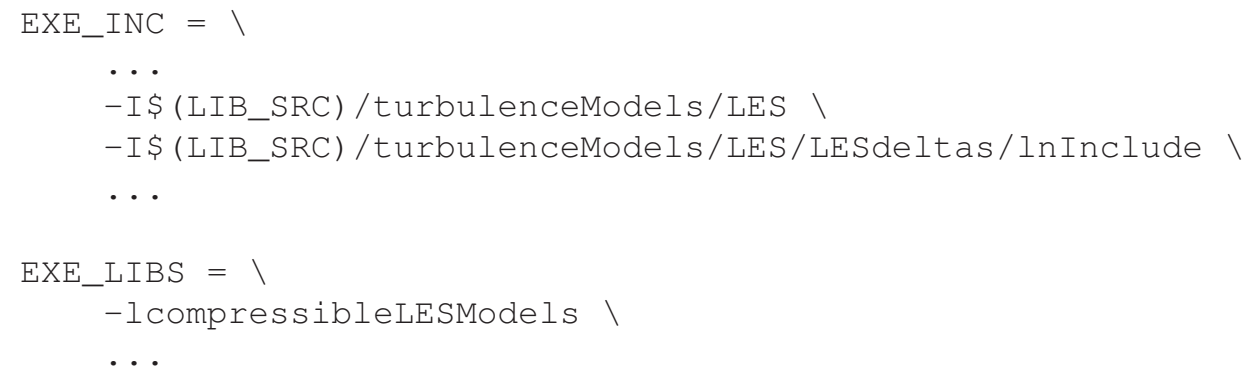

The second change is rebuilding the link from dieselLES to dieselEngineLES as following:

$1 \quad$ EXE_INC $=$ ।

2 -I../dieselEngineLES ।

The recompile the dieselEngineLES and dieselLES solver by running the command wmake under the folder dieselEngineLES and dieselLES.

The folder dieselLES contains two source code file, dieselLES.C and pEqn.H. In dieselLES.C, the following line redefined divDevRhoReff as divDevRhoBeff. The former is used in RANS to calculate the convection term in momentum equation, while the later is used in LES.

1 \#define divDevRhoReff divDevRhoBeff

The folder dieselEngineLES contains one file createFields.H which created the LESModel as the turbulence model. 


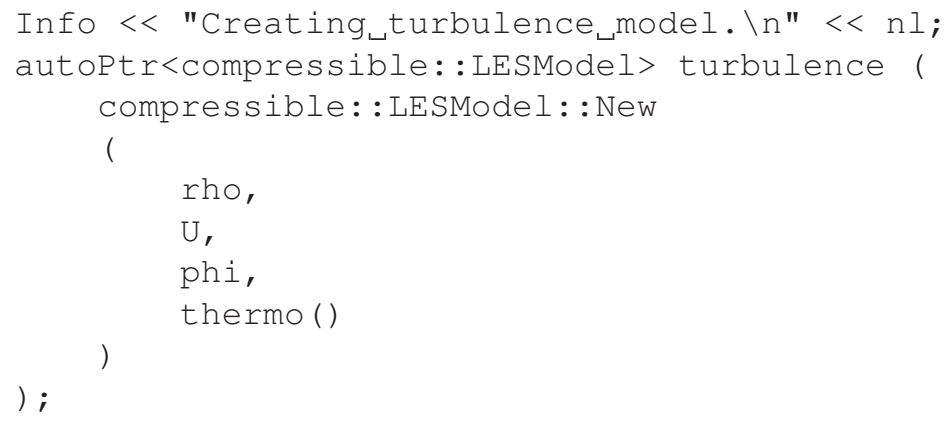

\section{A.3 New Fuel Realization}

In Chapter 5, the interested fuels, n-heptane, EN 590 and HVO, are not available in the fuel library of OpenFOAM. Hence, it is necessary to realize these fuels into the OpenFOAM fuel library. The properties of the fuels include the thermal physical properties and the chemical reaction properties. In this work, only non-evaporating and evaporating sprays are considered without the chemial reactions. Hence, the chemical reaction properties of the fuels are not interested. Details about the chemical reaction properties refer to Ref. ${ }^{98}$

Table A.1

Temperature Independent Thermal Physical Properties

\begin{tabular}{ccc}
\hline Sign & Name & Units \\
\hline$W$ & Molecular weight & {$[\mathrm{kg} / \mathrm{kmol}]$} \\
$T_{c}$ & Critical temperature & {$[\mathrm{K}]$} \\
$P_{c}$ & Critical pressure & {$[\mathrm{Pa}]$} \\
$V_{c}$ & Critical volume & {$\left[\mathrm{m}^{3} / \mathrm{mol}\right]$} \\
$Z_{c}$ & Critical compressibility factor & {[]} \\
$T_{t}$ & Triple point temperature & {$[\mathrm{K}]$} \\
$P_{t}$ & Trip point pressure & {$[\mathrm{Pa}]$} \\
$T_{b}$ & Normal boiling temperature & {$[\mathrm{K}]$} \\
dipm & Dipole moment & {[]} \\
$\omega$ & Pitzer's ascentric factor & {[]} \\
$\delta$ & Solubility parameter & {$\left[J^{1 / 2} / \mathrm{m}^{3 / 2}\right]$} \\
\hline
\end{tabular}




$$
\begin{aligned}
& \text { Density } \quad \rho=\frac{a}{b^{1+(1-T / c)^{d}}} \\
& \text { Vapor pressure } \quad p_{v}=\quad e^{a+b / T+c \ln (T)+d T^{e}} \\
& \text { Heat of vaporization } \quad h_{l}=a\left(1-T_{r}\right)^{b+c T_{r}+d T_{r}^{2}+e T_{r}^{3}}, T_{r}=T / T_{c} \\
& \text { Specific heat } \quad c_{p}=a+b T+c T^{2}+d T^{3}+e T^{4}+f T^{5} \\
& \text { Enthalpy } \quad h=a+b T+c T^{2}+d T^{3}+e T^{4}+f T^{5} \\
& \text { Ideal gas heat capacity } \quad c p g=a+b\left(\frac{c}{T \sinh (c / T)}\right)^{2}+d\left(\frac{e}{T \cosh (e / T)}\right)^{2} \\
& \text { Second viral coefficient } \quad B=\quad a+\frac{b}{T}+\frac{c}{T^{3}}+\frac{d}{T^{8}}+\frac{e}{T^{9}} \\
& \text { Dynamic viscosity } \quad \mu=e^{a+b / T+\operatorname{cln}(T)+d T^{e}} \\
& \text { Vapor dynamic viscosity } \quad \mu_{g}=\frac{a T^{b}}{1+\frac{c}{T}+\frac{d}{T^{2}}} \\
& \text { Thermal conductivity } \quad K=a+b T+c T^{2}+d T^{3}+e T^{4}+f T^{5} \\
& \text { Vapor therm. conduct. } \quad K_{g}=\quad \frac{a T^{b}}{1+\frac{c}{T}+\frac{d}{T^{2}}} \\
& \text { Surface tension } \quad \sigma=a\left(1-T_{r}\right)^{b+c T_{r}+d T_{r}^{2}+e T_{r}^{3}}, T_{r}=T / T_{c} \\
& \text { Vapor diffusivity } \quad D=\frac{3.6059 e-3(1.8 T)^{1} .75 \sqrt{1 / W_{f}+1 / W_{a}}}{p\left(a^{1 / 3}+b^{1 / 3}\right)^{2}}
\end{aligned}
$$

The thermal physical properties of the fuels, such as HVO, are documented in the file

$$
\text { \$FOAM_SRC/thermophysicalModels/liquids/HVO/HVO.H, }
$$

This file documented 11 temperature independent properties (Table A.1), and 13 temperature dependent properties (Eqs.A.1-A.13). The temperature dependent properties are written as the functions of the temperature with the determined coefficents $T_{c}, a, b, c, d, e, f, W_{f}$ and $W_{a}$. These functions are given in the folder

\$FOAM_SRC/thermophysicalModels/thermophysicalFunctions.

In the HVO.H file,

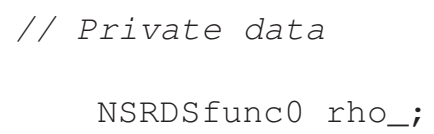




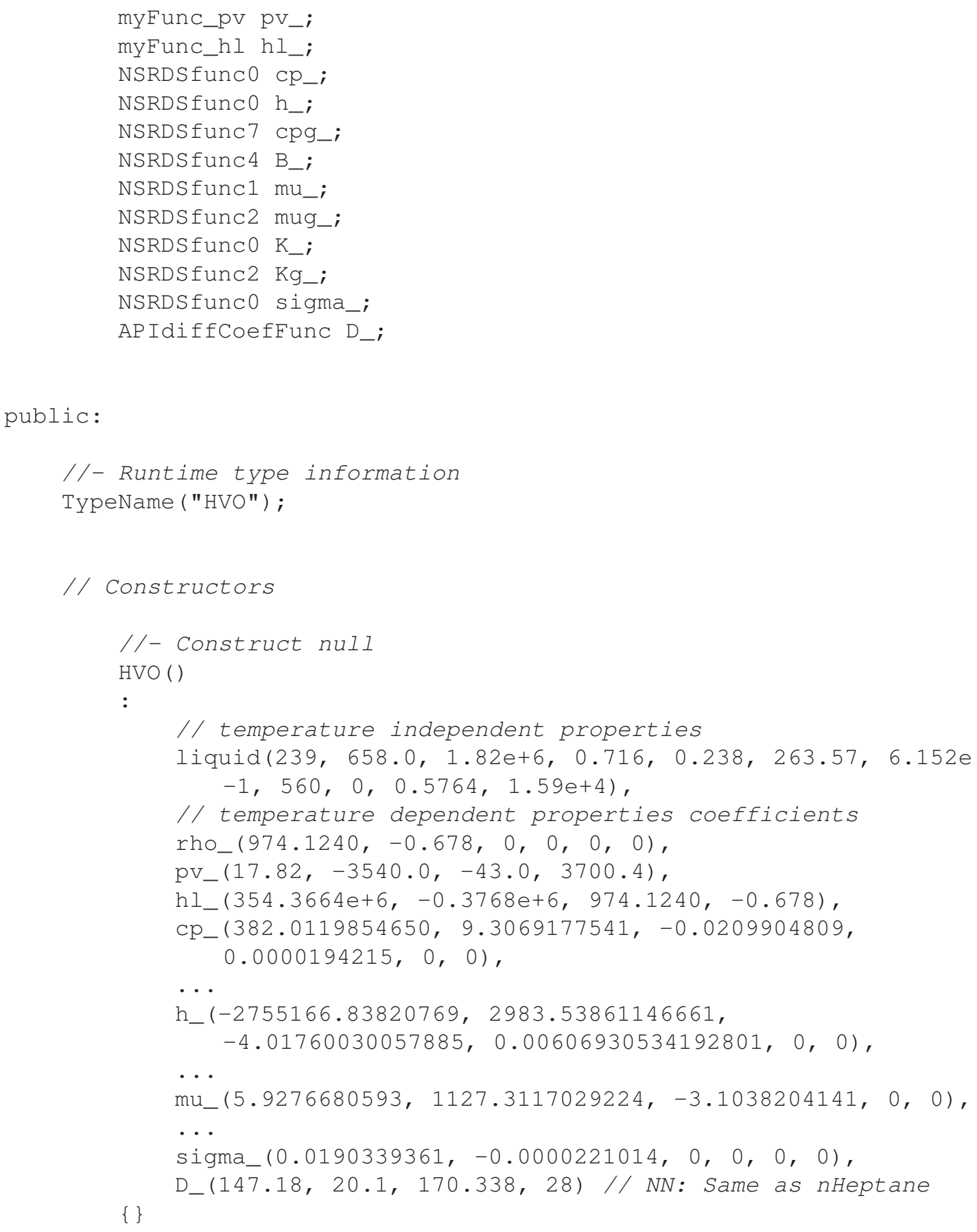

the 30rd line provides the temperature independent properties listed as the order in Table A.1. The lines from the 32th line to the 42th line give the coefficients, $T_{c}, a, b, c, d, e, f, W_{f}$ and $W_{a}$ of the temperature dependent functions as shown in Eqs. A.1A.13. 


\section{A.4 Compile the user-defined libraries}

\section{A.4.1 Move an existed library to user's directory}

To build your own libraries without affecting the default OpenFOAM library, it is necessary to copy the library source files from the original position to the user's directory. For example, the following command moves the default dieselSpray library to a new position.

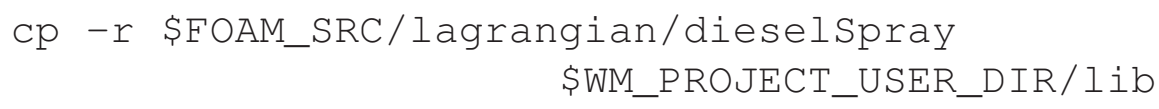

Then modify the compilation files in the Make folder.

cd \$WM_PROJECT_USER_DIR/lib/Make

There are two files and one folder in this folder. First change the last line of files from

$$
\mathrm{LIB}=\text { \$(FOAM_LIBBIN)/IibdieselSpray }
$$

to

$$
\text { LIB }=\text { \$ (FOAM_USER_LIBBIN)/IibmydieselSpray } \text {. }
$$

Then check the options file that whether the new library used some other new defined libraries. If yes, then add the user's own library folder and the new library name to LIB_LIBS. For example, here we need to use a new defined mycompressibleRASModels library. Then change Make/options file as following.

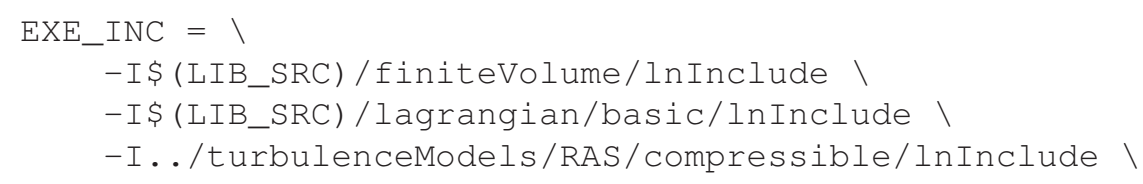




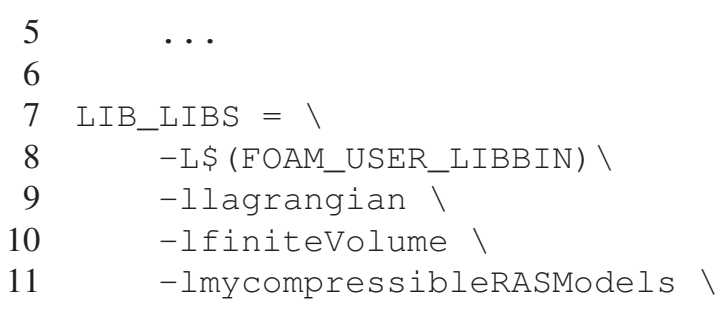

Lastly, recompile the new library

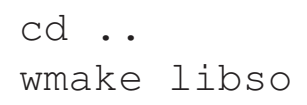

Check the user's library folder

$$
\$ F O A M \backslash U S E R \backslash L I B B I N \text {, }
$$

there should contain the new library file called libmydieselSpray.libso or libmydieselSpray.dylib. The filename extension is decided by different operation systems. In linux system, the extension is .libso, while in osx system, it is .dylib.

\section{A.4.2 Add a new library or library components}

It is often required to build a new library, or a new component of one exist library in OpenFOAM. For example, Sec A.1.3 needs to add the CAB breakup model to the librarydieselSpray. Before the recompilation of the new library as in Sec A.4, a series steps are done as below.

Firstly, make a new folder $\mathbf{C A B}$, and copy all files in ETAB folder to $\mathbf{C A B}$.

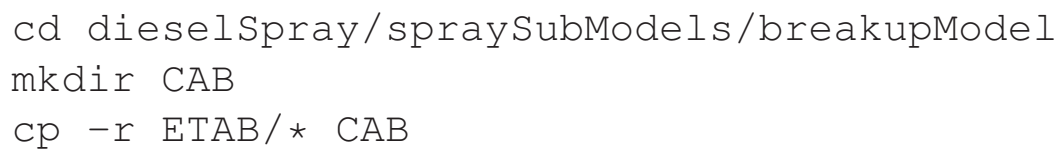

Secondly, change all the strings ETAB to $\mathbf{C A B}$. 


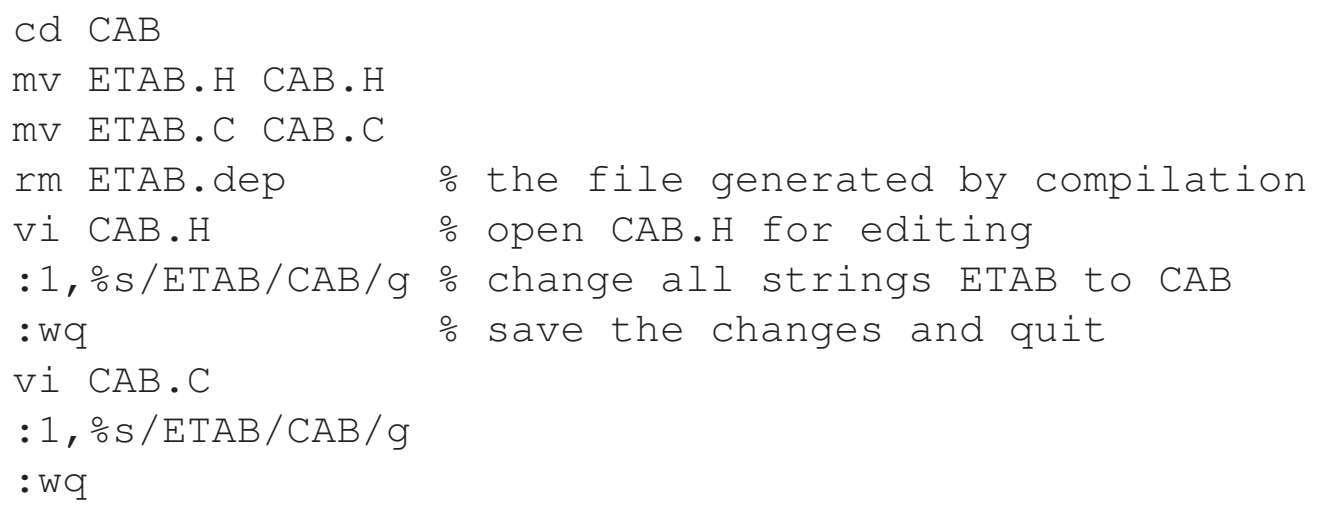

Thirdly, modify the CAB.C file according to the CAB model in Sec A.1.3.

Fourthly, add the $\mathrm{CAB}$ model into the compilation list.

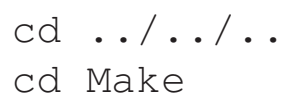

Then, add the following line to the file files

\$ (breakupModels) / CAB / CAB . C

Lastly, recompile the new library

$\mathrm{cd}$. .

wmake libso 


\section{B Spray source term}

One of the shortcomings of OpenFOAM is the lack of a direct coupling between the turbulence equations and the spray droplets. To remedy this problem, appropriate source terms have been implemented into the transport equations for the turbulence kinetic energy, $k$, and its dissipation rate, $\varepsilon$. These source terms account for the amount of turbulent energy that is dissipated because of the work performed on the droplets by the turbulence velocity fluctuations. The effect of these turbulence model source terms are evaluated by looking at the turbulent kinetic energy and at the turbulence viscosity $\mu_{t}$.

\section{B.1 Introduction}

To model the effect of the droplet spray over the turbulence, a spray source term is introduced into the RNG $k-\varepsilon$ turbulence model. With the current level of averaged RANS modeling, the mechanism by which the droplets interact directly with turbulence can be considered to occur from turbulence that does work onto droplets, or by turbulent droplet motions producing turbulence directly. The former mechanism is modeled in many RANS codes by turbulent dispersion models which try to mimic the effect of small scale turbulence onto droplets by introducing an additional random velocity component for the droplets. Here, the work done to the turbulent flow field from the droplets is modeled by the separate source term in the turbulence equations.

The RNG $k-\varepsilon$ turbulence model with the spray source term has the following form ${ }^{47}$

$$
\begin{aligned}
\mu_{t} & =C_{\mu} \rho k^{2} / \varepsilon \\
\frac{\partial \rho k}{\partial t}+\nabla \cdot(\rho \mathbf{u} k) & =\nabla \cdot \mu_{\alpha} \nabla k+\mu S^{2}-\rho \varepsilon+S_{k} \\
\frac{\partial \rho \varepsilon}{\partial t}+\nabla \cdot(\rho \mathbf{u} \varepsilon) & =\nabla \cdot \mu_{\alpha} \nabla \varepsilon-\left(\frac{2}{3} C_{\varepsilon 1}-C_{\varepsilon 3}\right) \rho \varepsilon \nabla \cdot \mathbf{u}+\frac{\varepsilon}{k}\left[C_{\varepsilon 1}^{*} \mu_{t} S^{2}-C_{\varepsilon 2} \rho \varepsilon+C_{S} S_{k}\right],
\end{aligned}
$$

where $\mu_{t}$ denotes the turbulent viscosity with a default constant $C_{\mu}=0.0845, S$ represents the modulus of the mean rate-of-strain tensor defined as $S=\sqrt{2 S_{i j} S_{i j}}, \mu_{\alpha}=\alpha_{k} \mu_{t}+\mu$ with $\alpha_{k}=1.39, C_{\varepsilon 2}=1.92, C_{\varepsilon 3}=-1.0, C_{s}=1.5, C_{\varepsilon 1}^{*}=C_{\varepsilon 1}-C_{\eta}$ with $C_{\varepsilon 1}=1.44$ and $C_{\eta}=\eta\left(1-\eta / \eta_{0}\right) /\left(1+\beta \eta^{3}\right)$ with $\eta_{0}=4.38, \beta=0.012$ and $\eta=S k / \varepsilon$. 
The spray source term $S_{k}$ accounts for the modification of turbulence by the droplets. Regarding all the contact forces as unity, the widely used Lagrangian form of the spray source term $S_{k}$ in the k-equation has the simple form ${ }^{47,106}$

$$
S_{k}=\overline{\mathbf{u}^{\prime} \cdot \mathbf{F}}
$$

where the average is performed over the interface between the droplets and the fluid field, $\mathbf{u}^{\prime}$ denotes the fluid velocity fluctuation at the droplet position, $\mathbf{F}$ are all the contact forces over the interface.

Despite the fact that this term has been discussed in Refs., ${ }^{67,99,106,143}$ most realizations are based on simplified assumptions, some of which only dissipate the turbulent kinetic energy. Moreover, this source term is often ignored in CFD codes, including OpenFOAM. In this study, a plain derivation of this source term is used and implemented into OpenFOAM. By the Newton's third law, the contact force exerted on the fluid by a droplet is the opposite of the force acting on this droplet. By Newton's second law, the contact force is proportional to the droplet acceleration, i.e., to the time rate change of the droplet velocity. Hence, $\mathbf{F}=-m_{d} \dot{\mathbf{u}}_{d}$. For any droplet, the discrete time rate change of the velocity is

$$
\frac{d \mathbf{u}_{d}}{d t}=\frac{\mathbf{u}_{d}(t+\Delta t)-\mathbf{u}_{d}(t)}{\Delta t}
$$

Inserting Eq. B.3 into Eq. B.2, the discrete form of the spray source term $S_{k}$ in any computational cell cellI, is written as:

$$
S_{k}[c e l l I]=\sum_{d}\left(m_{d}^{o} \mathbf{u}^{\prime} \cdot \mathbf{u}_{d}^{o}-m_{d}^{n} \mathbf{u}^{\prime} \cdot \mathbf{u}_{d}^{n}\right) /\left(V_{c e l l I} \Delta t\right),
$$

where the superscripts ${ }^{o}$ and ${ }^{n}$ represent the value at time $t$ and time $t+\Delta t$, respectively, $m_{d}$ denotes the droplet mass and $V_{\text {cell }}$ is the cell volume. The velocity fluctuation $\mathbf{u}^{\prime}$, given by the dispersion model, is defined as

$$
\mathbf{u}^{\prime}=\gamma \mathbf{I},
$$

where $\mathbf{I}$ is a random unit vector, and $\gamma \sim N(0, \sigma)$ is a Gaussian random number with variance $\sigma=\sqrt{2 k / 3}$. 


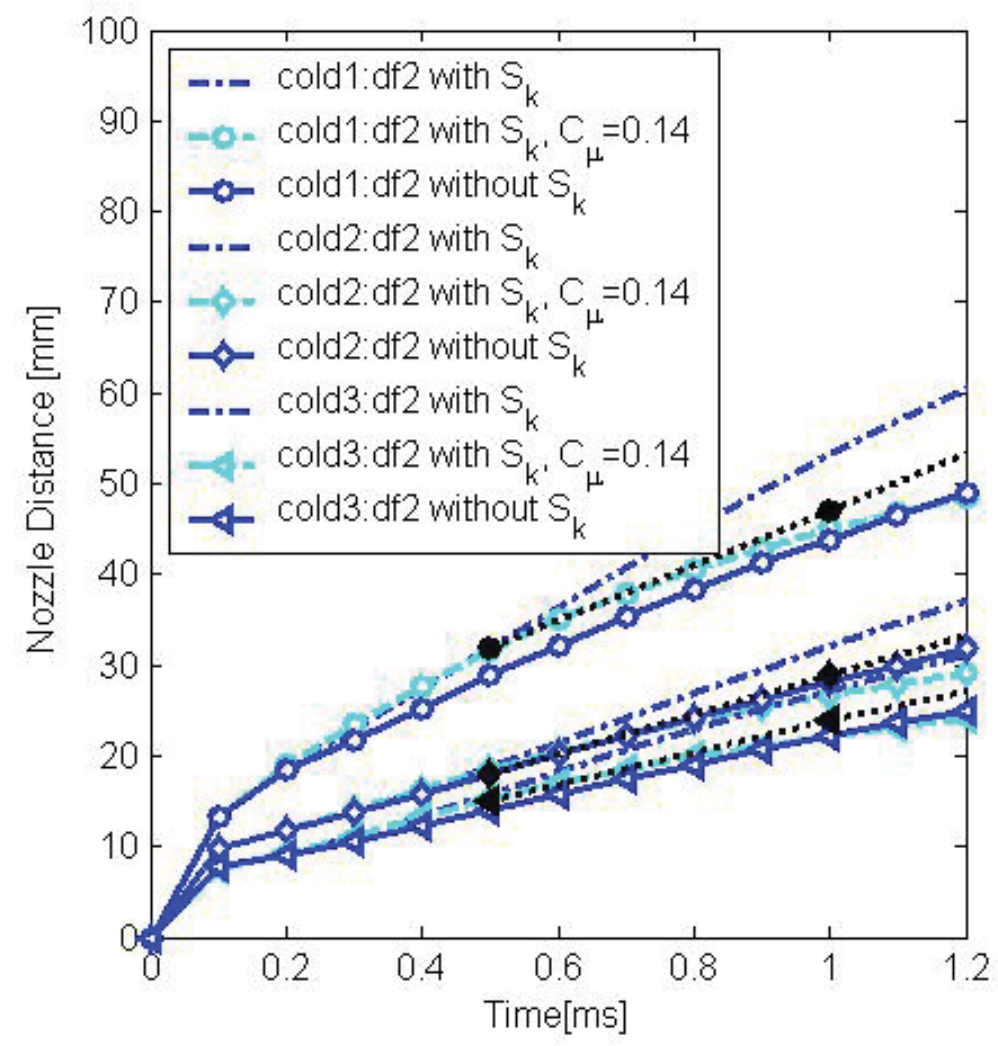

Figure B.1: Spray tip penetration versus time of the non-evaporating cases for DF2.

\section{B.2 Simulations and Results}

The simulation are done on the non-evaporating DF2 cases and evaporating HEP case in Table 4.1 as in Chapter 4. The comparisons are done using the RNG $k-\varepsilon$ turbulence model with or without the spray source terms.

The effect of the spray source term $S_{k}$ on the turbulent kinetic energy $k$ and the turbulent viscosity $\mu_{t}$ are plotted for the non-evaporating DF2 cases in Fig. B.2 and Fig. B.3 along the spray axis at $0.6 \mathrm{~ms}$ and $1.2 \mathrm{~ms}$ after the start of injection. As is seen in these figures, the peaks of the turbulent kinetic energy and the turbulent viscosity are reduced when the source term was switched on $\left(S_{k} \neq 0\right)$. This is consistent with the fact that the turbulence velocity fluctuations perform work on the droplet, and hence reduce the turbulent kinetic energy of the gas. The overall reduction of the turbulent viscosity $\mu_{t}$ shown in Fig. B.3 results in an overestimation of the spray penetration, as is illustrated in Fig. B.1. 

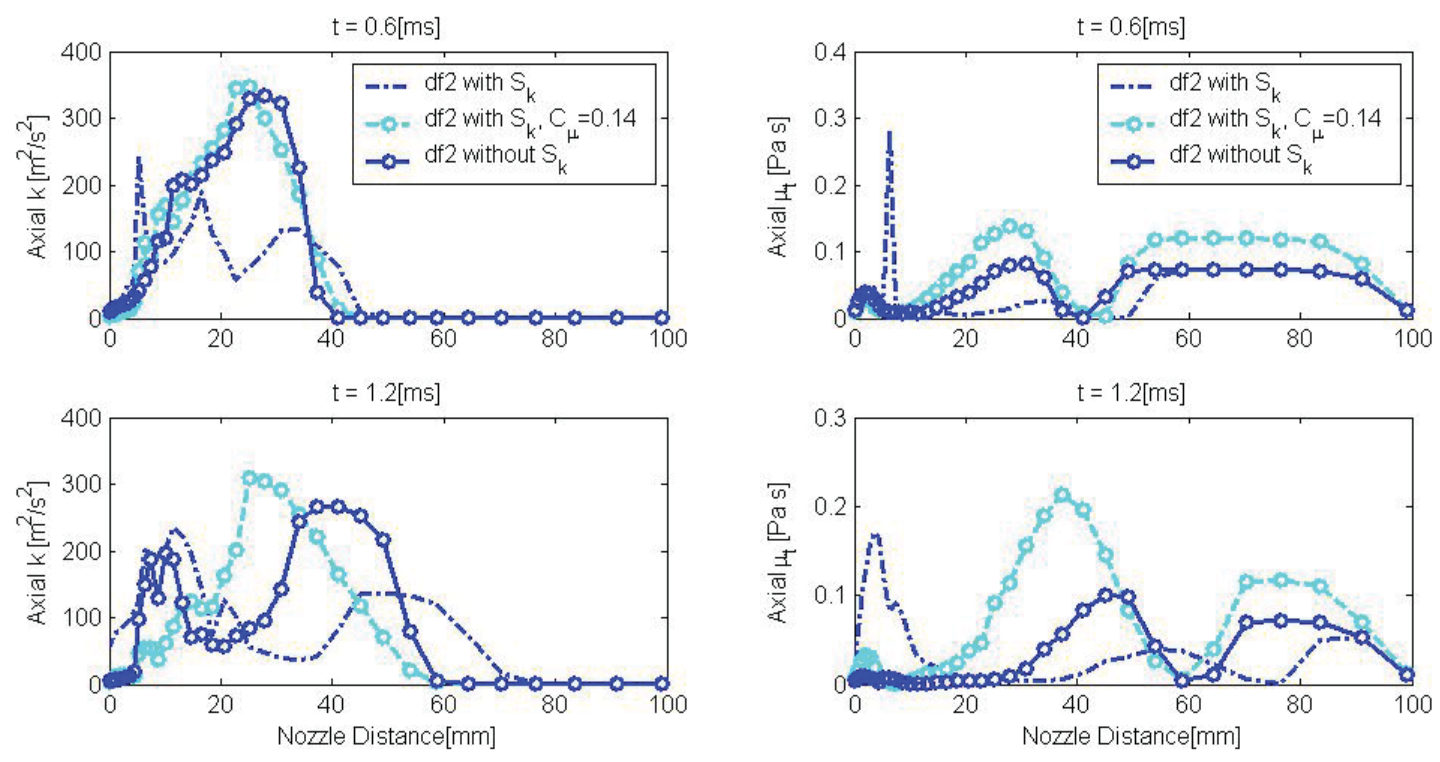

Figure B.2: Axial turbulent kinetic energy $k$ of the non-evaporating Case 1 for DF2.

Figure B.3: Axial turbulent viscosity $\mu_{t}$ of the non-evaporating Case 1 for DF2.

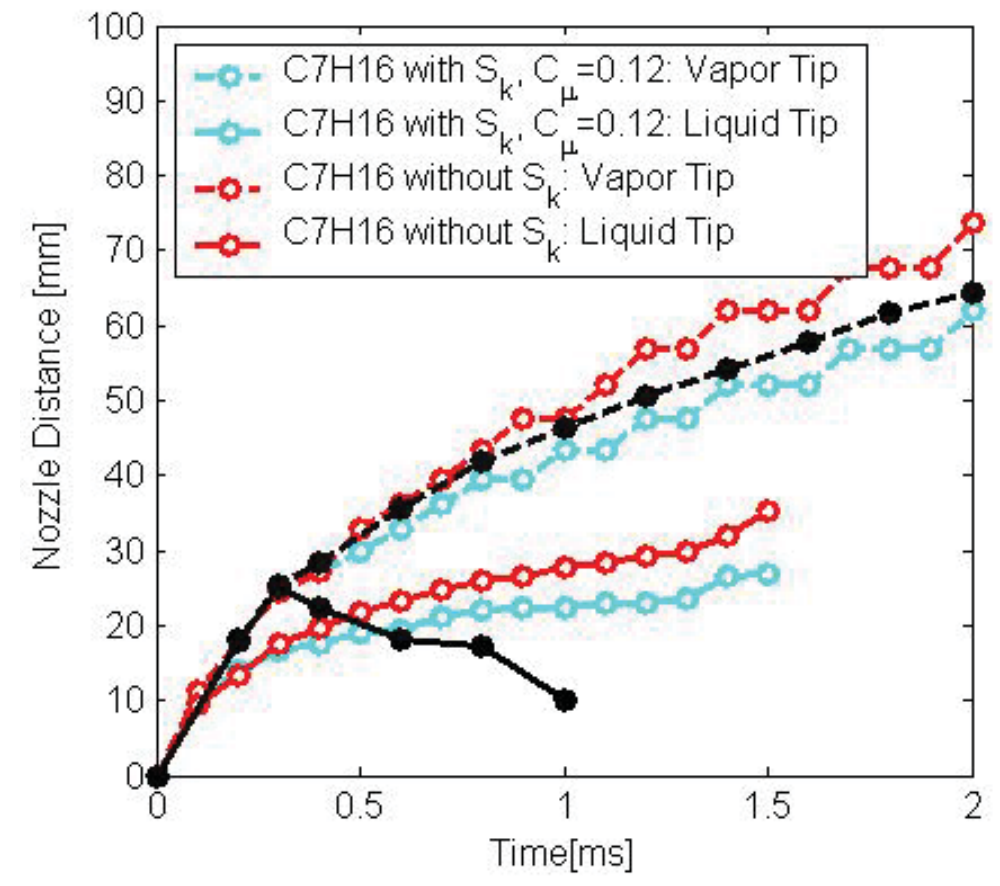

Figure B.4: Vapor penetrations (dashed lines) and liquid penetrations (solid lines) versus time of the evaporating case for HEP. 

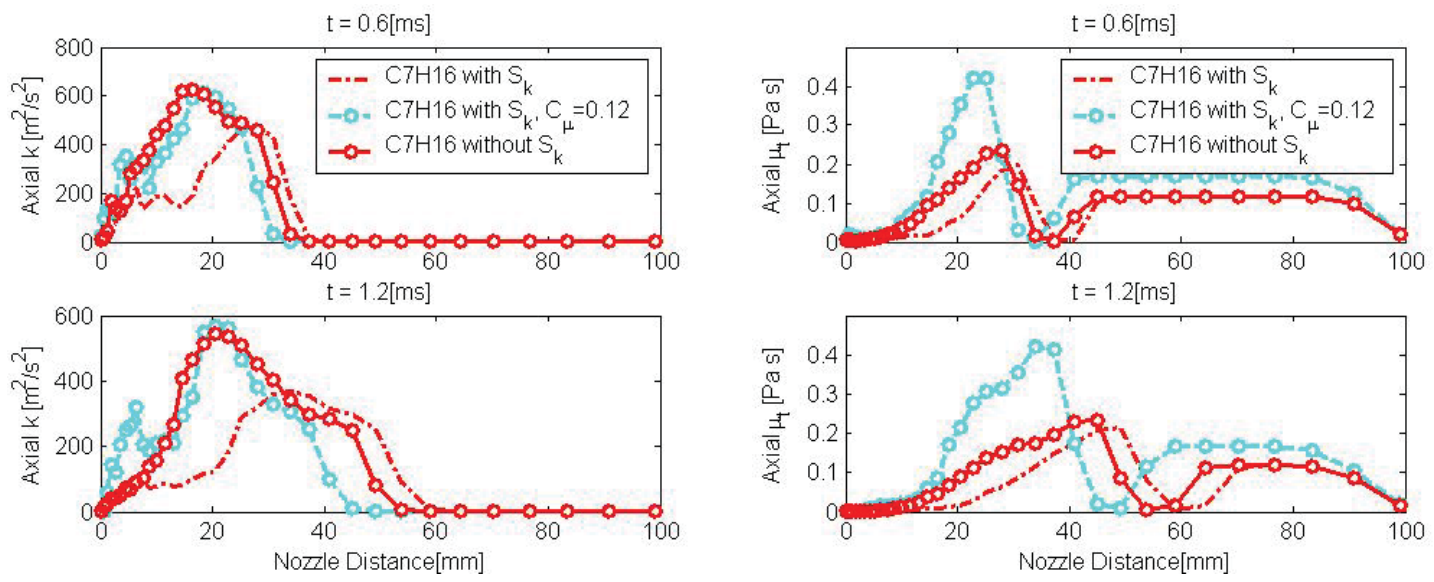

Figure B.5: Axial turbulent kinetic energy k of the evaporating case for HEP .

Figure B.6: Axial turbulent viscosity $\mu_{t}$ of the evaporating case for HEP.

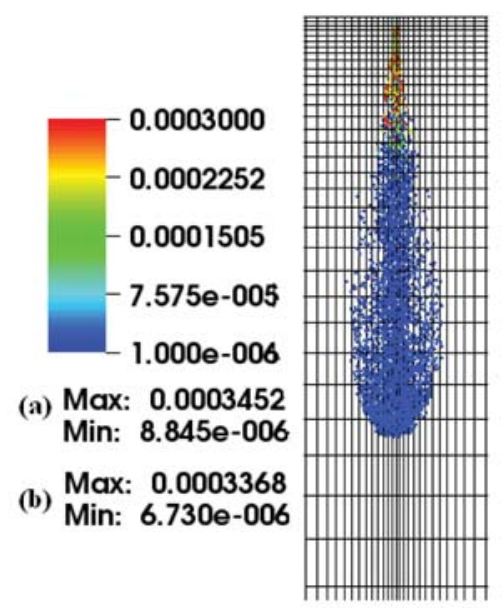

(a)

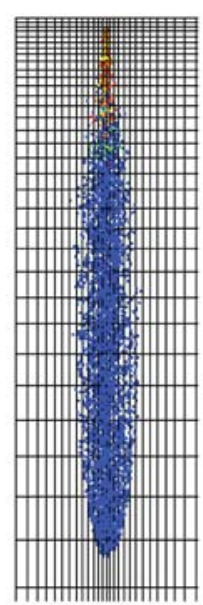

(b)

Figure B.7: Spray droplet diameter in $\mathrm{m}$ of the non-evaporating Case 1 using DF2 fuel at 0.0012 s: (a) $S_{k}=0$; (b) $S_{k} \neq 0$.

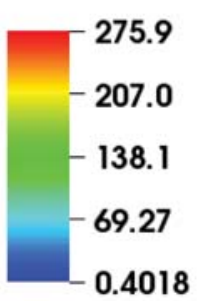

(a) Max: 275.9 Min: 0.4018

(b) Max: 232.3 Min: 0.4021

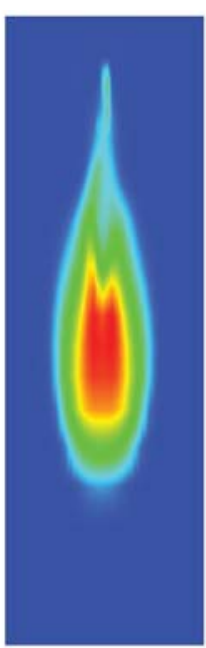

(a)

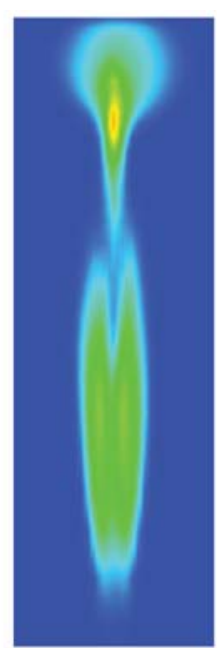

(b)
Figure B.8: Turbulent kinetic energy in $\mathrm{m}^{2} / \mathrm{s}^{2}$ of the non-evaporating Case 1 using DF2 fuel at 0.0012 s: (a) $S_{k}=0$; (b) $S_{k} \neq 0$.

For the evaporating spray case, the effect of the source term $S_{k}$ on the penetrations, the turbulent kinetic energy and the turbulent viscosity is almost the same as in the nonevaporating case. The results are shown in Figs. B.4-B.6.

In addition, Figs. B.7-B.8 show the modulation of the turbulent kinetic energy more clearly: the turbulent kinetic energy is extremely large in the center of spray if $S_{k}=0$, while the modified RNG $k-\varepsilon$ model with $S_{k} \neq 0$ depletes the turbulent kinetic energy in the spray 
center and allocates more turbulent kinetic energy to the spray tip and the liquid core near the nozzle.

\section{B.3 Discussion}

For both, the non-evaporating cases and the evaporating case, adding the spray source term to the turbulence equations reallocated and depleted the turbulent kinetic energy. As studied in Chapter 4, RANS overpredict the turbulent kinetic energy than LES. Hence, it is interested to realize the spray source term into OpenFOAM for future studies with RANS simulations.

However, the depletion of the turbulent kinetic energy resulted in the increasement of the penetration curve. Thus to lower the penetration curve to the level of the experimental result, a tuning of the turbulent constant $C_{\mu}$ is done in this study. Considered the randomness and unpredictable errors in tuning the constants, this work uses the OpenFOAM original $k-\varepsilon$ and RNG $k-\varepsilon$ equations without the spray source terms in Chapter 4 and Chapter 5.

Additionally, from Eq. B.4, the spray source term $S_{k}$ is comparable negligible in the LES where the turbulent kinetic energy are much smaller than the RANS. Hence, usually, the influence of the spray to the LES turbulence equation is not considered.

\section{B.4 OpenFOAM Realization}

In OpenFOAM, the spray source term is realized in the following steps:

1. Add a subfield sks to dieselSpray class, which store the value of the spray source term in each cell.

2. Add a member function updateSpraySource to class RASModel, which delivers the spray source term value from dieselSpray to turbulence equations.

3. Modify the turbulence $k-\varepsilon$ equations to include the spray source term sks_. 


\section{B.4.1 Add a subfield sks to dieselSpray class}

Copy the folder dieselSpray to the user's library.

$$
\begin{aligned}
& c P-r \text { \$FOAM_SRC/lagrangian/dieselSpray } \\
& \text { \$WM_PROJECT_USER_DIR/Iib }
\end{aligned}
$$

Make the following changes, and then recompile the new library according to chapter A.4.1.

- In the spray/spray.H file, add a scalar field sks to the class declaration. The format of sks is similar to the existing field shs. Then sks will be defined in parcel/parcel.C file.

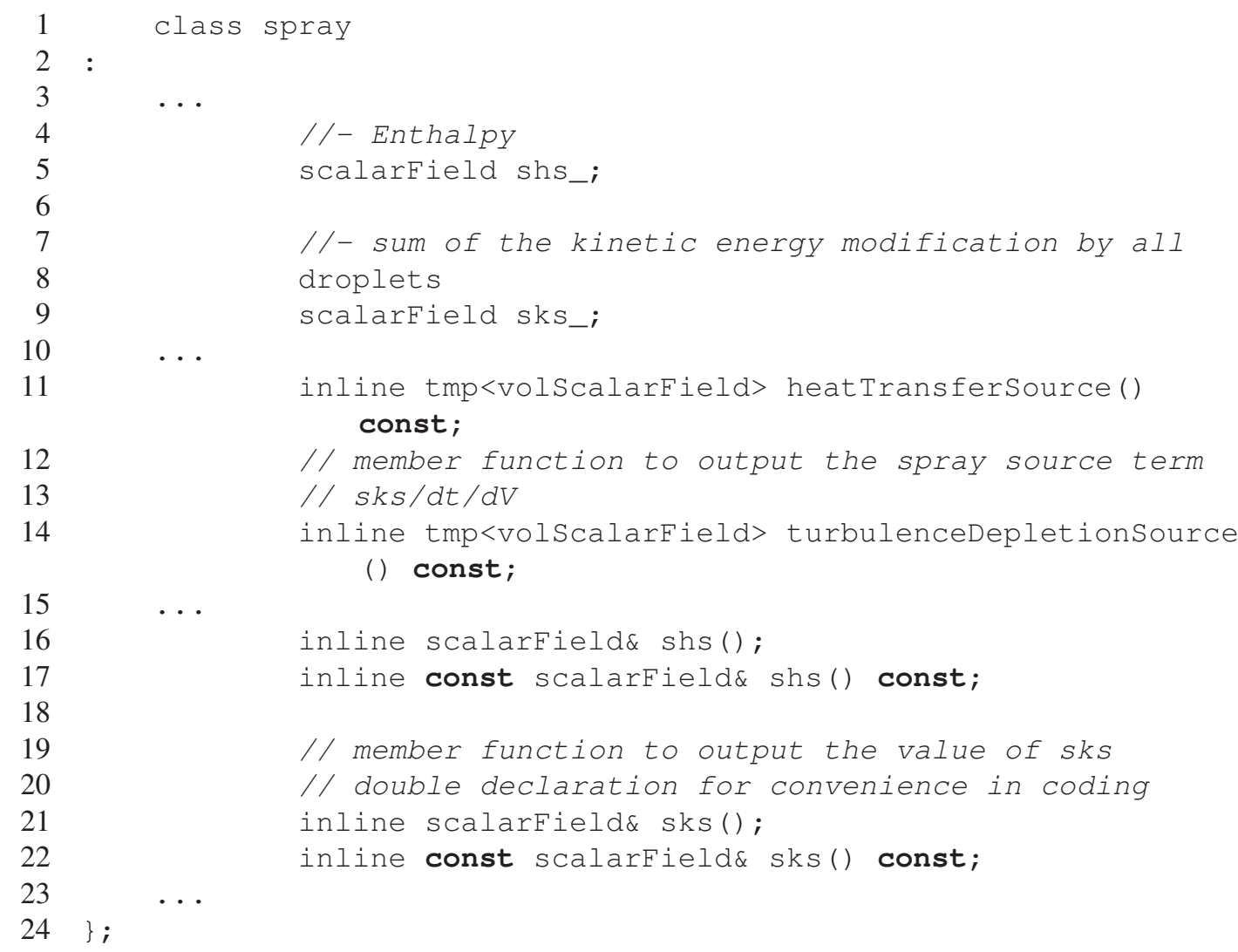

- In the spray/sprayI.H file, the new member functions introduced above are defined. Here, min(sks_,0.0) takes the negative part of sks_, and then $\max (. .$, ..) limited the value less than $-1 \mathrm{e}-6$. 


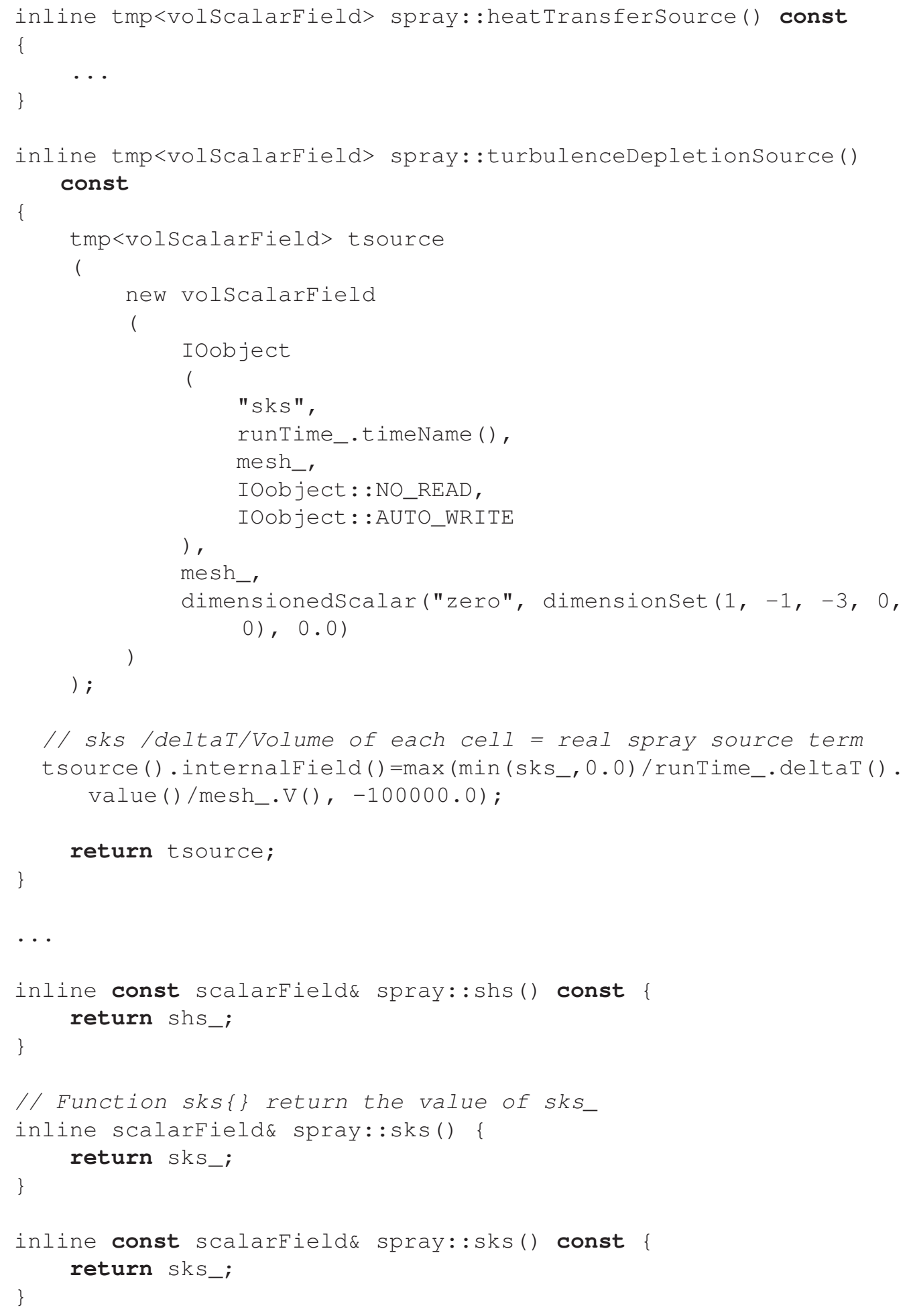

- In file spray/spray.C, initialize sks as a zero scalar, 
1 shs_(mesh_.nCells(), 0.0),

2 sks_(mesh_.nCells(), 0.0),

- In file spray/sprayOps.C, distribute free space to sks

1 shs_.setsize(rho_.size());

2 sks_.setsize(rho_.size());

and initialize sks in the member function, move

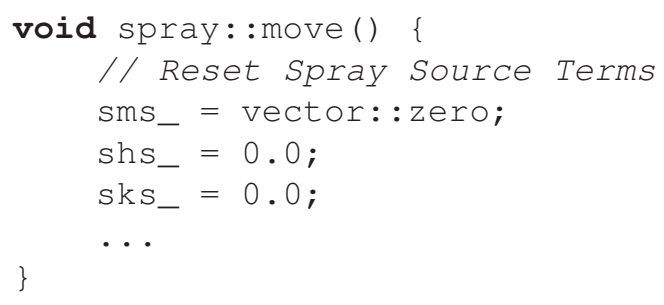

- In file parcel/parcel.C, calculate sks.

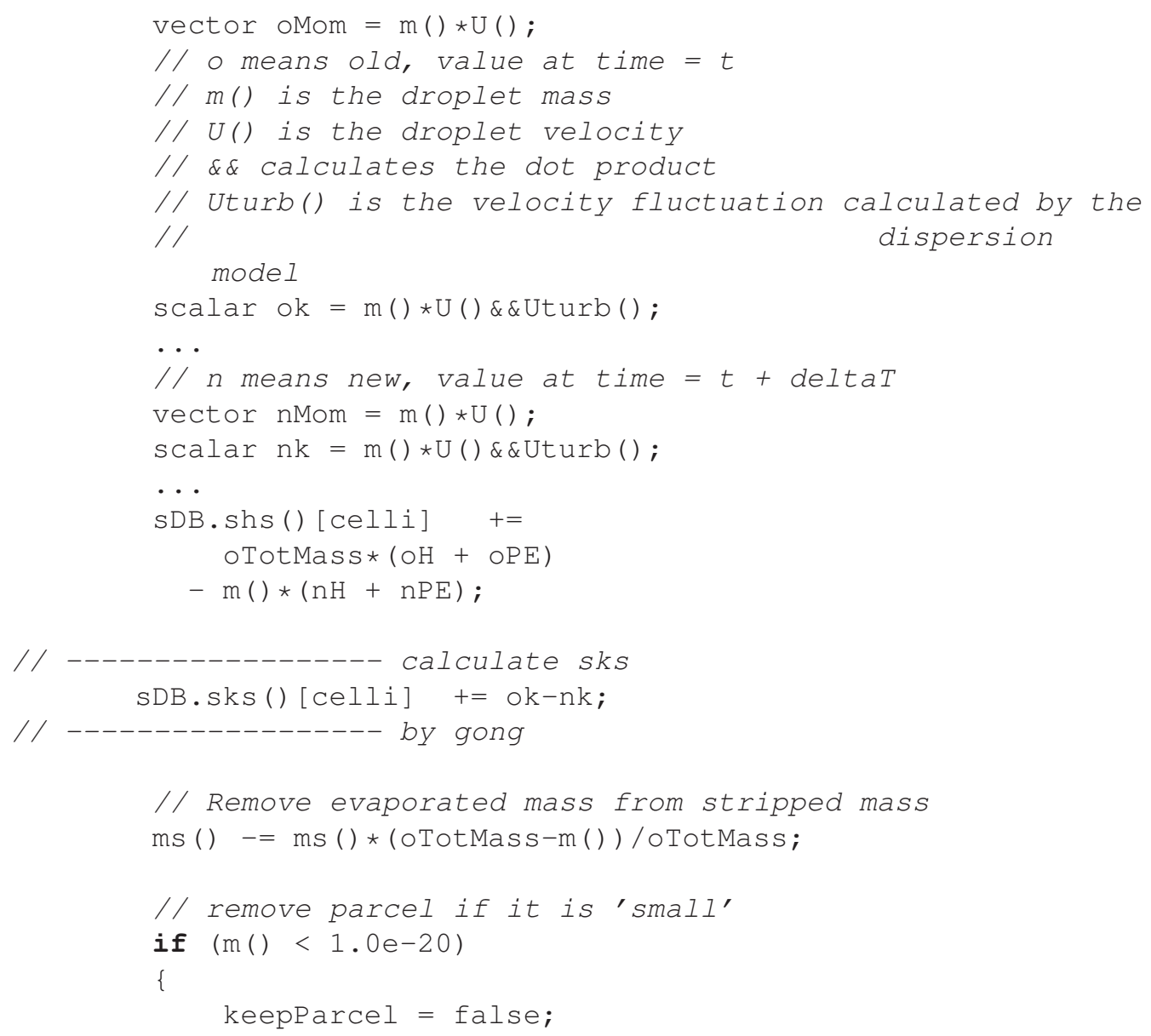




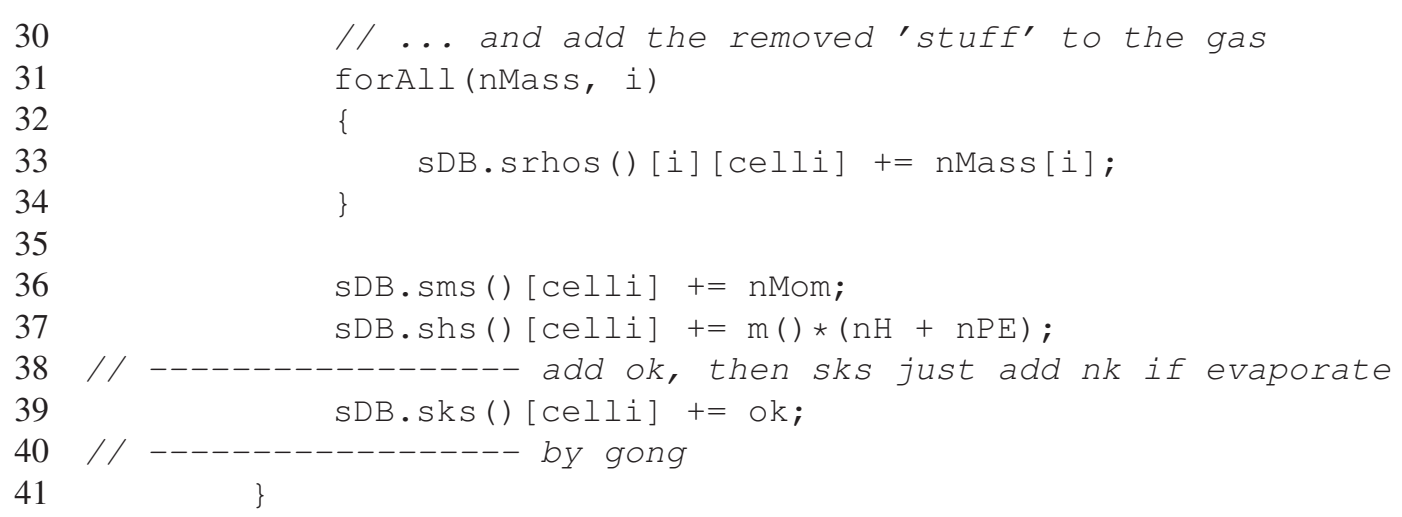

Here, the vector newUturb is the vector from the nozzle pointing to the parcel position. If the dot product of the generated random vector Uturb and newUturb is negative, then sks is forced to add a negative value, for that we do not expect to add too much kinetic energy to the inverse injection direction. Without this conditional statement, too many kinetic energy add redistributed to the near nozzle region and cause the crash of the code. Noticed that ok-nk is statistically negative, thus this limitation is acceptable but still needs further test.

\section{B.4.2 Add a member function updateSpraySource to class RASModel}

The spray source term calculated by the dieselSpray is delivered to the turbulence model by a member function called updateSpraySource.

First copy the folder turbulenceModels to the user's library.

$C P$ - $r$ \$FOAM_SRC/turbulenceModels \$WM_PROJECT_USER_DIR/lib

Follow the steps below, and then recompile the new library according to chapter A.4.1.

- In file RAS/compressible/RASModel/RASModel.H, add the declaration of the new member function updateSpraySource

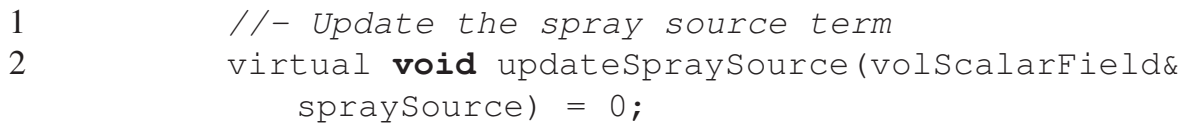


- Copy the contents of folder RNGkEpsilon to a new folder sprayRNGkEpsilon

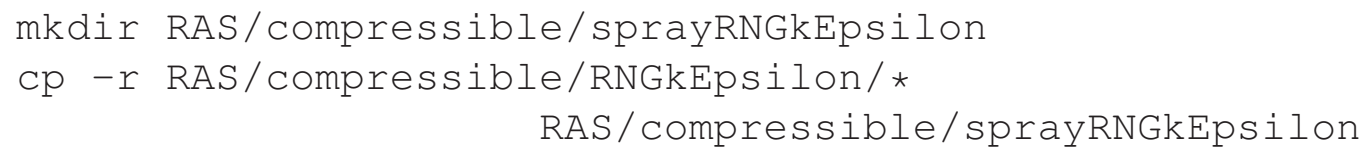

Add spray to the front of each file name, and change all RNGkEpsilon to sprayRNGkEpsilon in the file (the command lines given below is used in the vi editor).

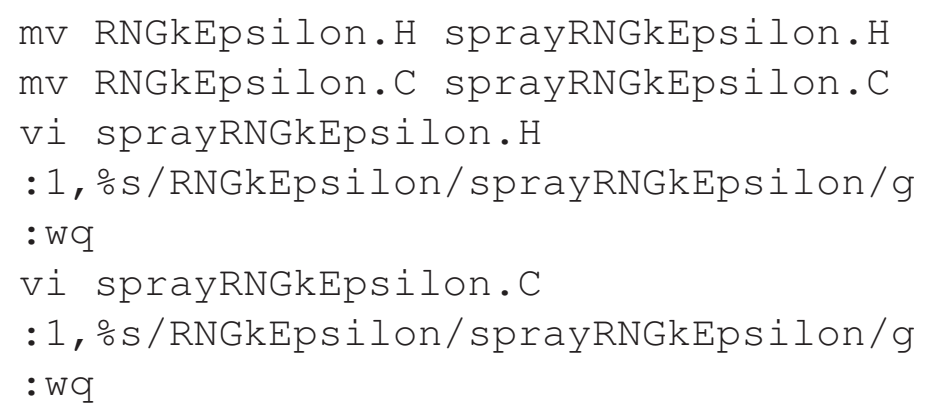

Add the file sprayRNGkEpsilon to file Make/files, then OpenFOAM will compile the new turbulence model sprayRNGkEpsilon if run the wmake libso command.

RNGkEpsilon/RNGkEpsilon.C

sprayRNGkEpsilon/sprayRNGkEpsilon.C

- In file RAS/compressible/sprayRNGkEpsilon/sprayRNGkEpsilon.H, also add the declaration of the new member function updateSpraySource

$1 \quad / /$ - Update $k$ and epsilon adding the spray source term

2 void updatespraysource (volscalarfield\& spraysource);

- In file RAS/compressible/sprayRNGkEpsilon/sprayRNGkEpsilon.C, define updateSpraySource.

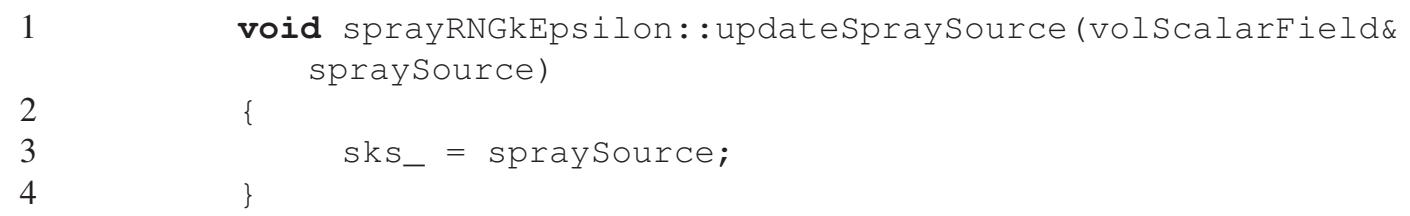

- Repeat the above two steps for all the other turbulence model classes, such as kEpsilon. This is a little troublesome. But all subclasses inherited all member functions of the mother class, so we need to include the new defined member function in all subclasses either. 


\section{B.4.3 Add the spray source term sks_ to $k-\varepsilon$ equations}

In the definition of the member function updateSpraySource, a new variable sks_ is used to represent the spray source term.

- The variable sks_ is defined in the file RAS/compressible/RNGkEpsilon/RNGkEpsilon.H

$1 \quad$ volscalarfield sks $\backslash_{-} ;$

- And sks is initialized in the constructor in the file RNGkEpsilon.C, which is in the folder RAS/compressible/RNGkEpsilon.

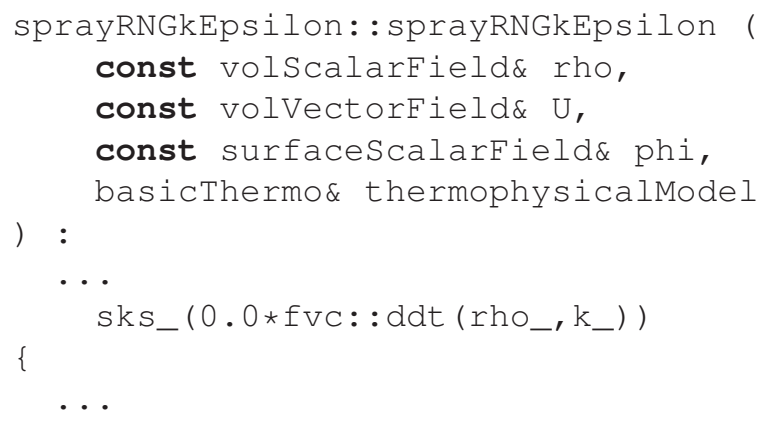

- Then sks_ is added to the epsEqn and kEqn in the file RNGkEpsilon.C, which is in the folder RAS/compressible/RNGkEpsilon.

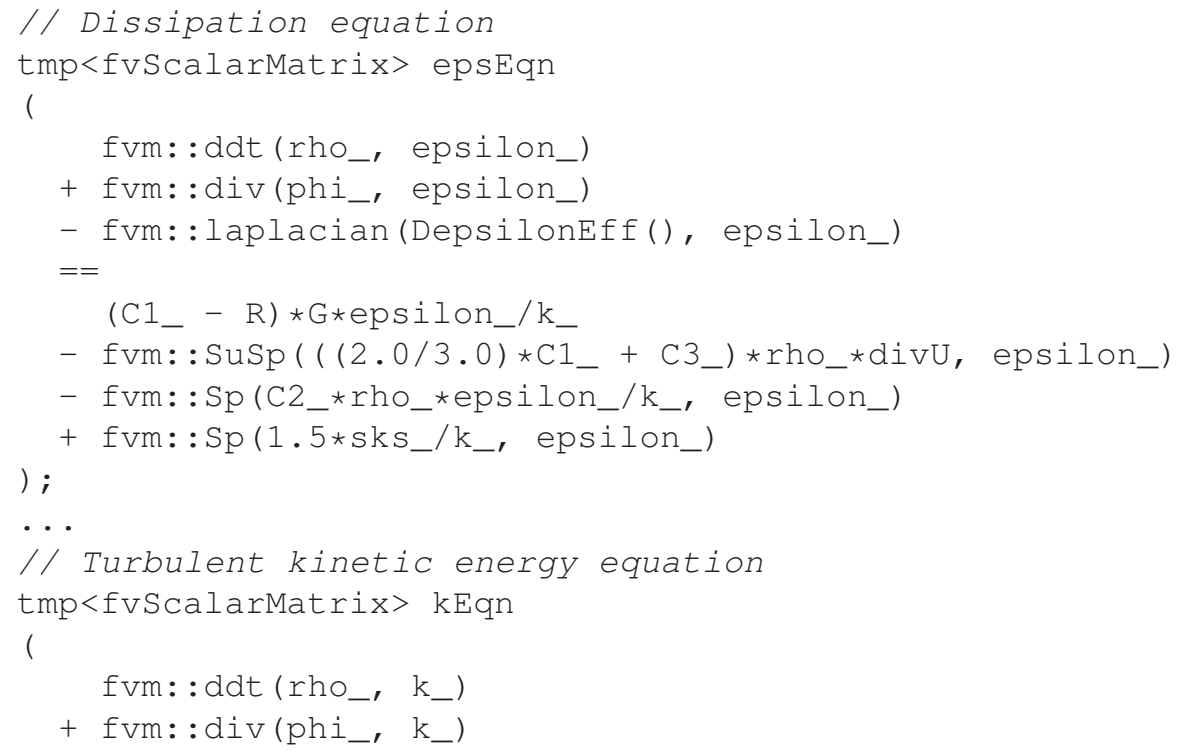




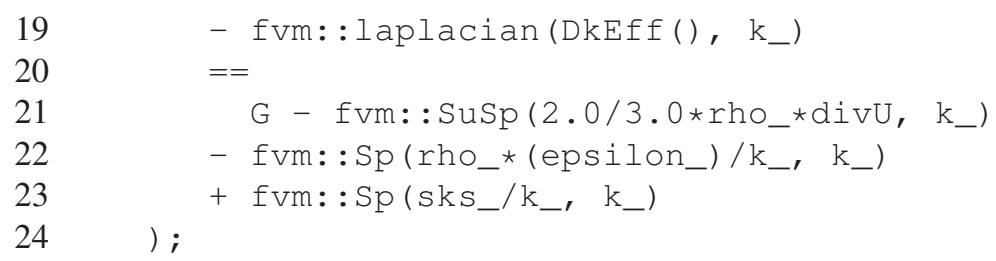

\section{B.4.4 Update the spray source term in solvers}

- Initialize the spray source term spraySrc at the beginning of the solver, myDieselFoam.C.

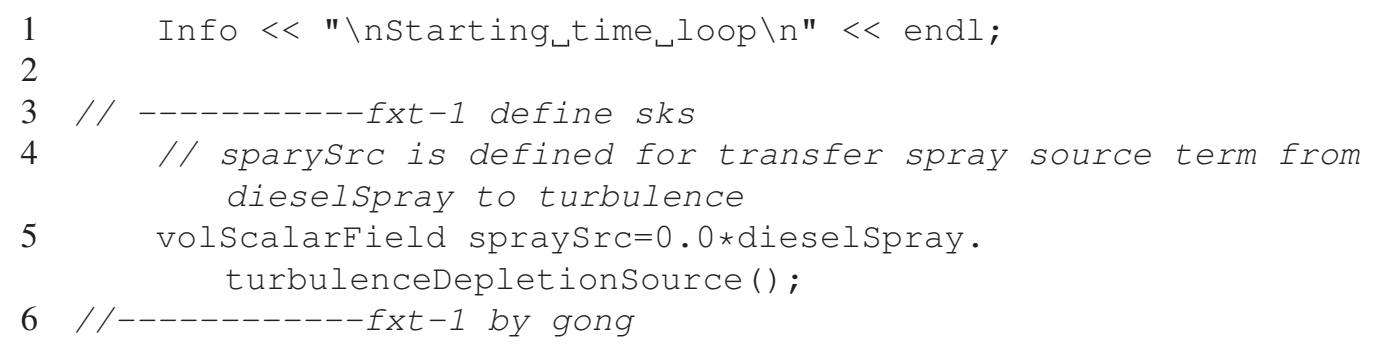

- Update spraySrc once done the dieselSpray updating.

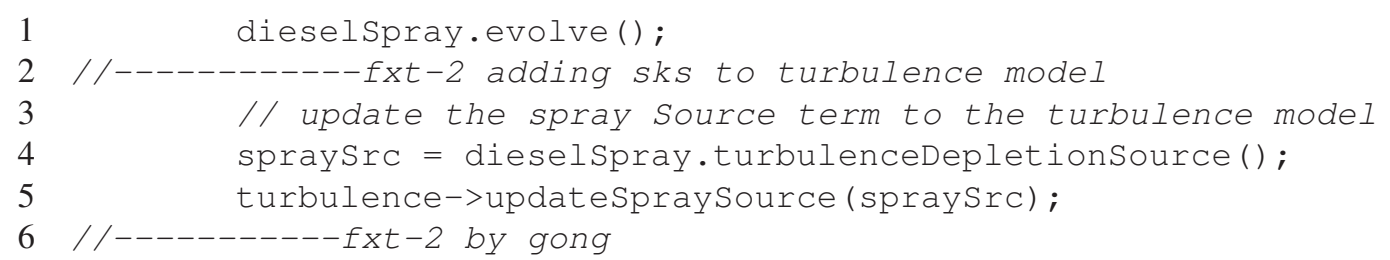

